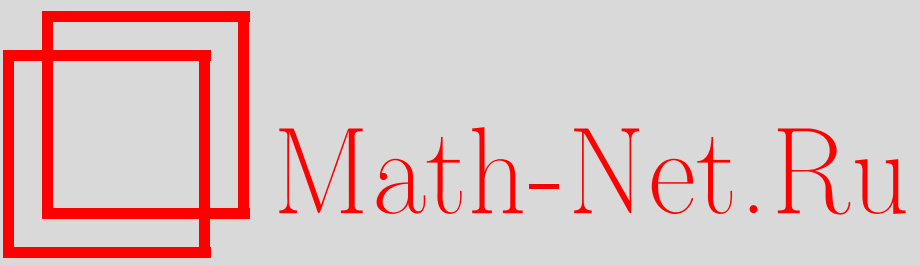

Б. М. Гуревич, С. В. Савченко, Термодинамический формализм для символических цепей Маркова со счетным числом состояний, УМН, 1998, том 53, выпуск 2, 3-106

DOI: https://doi.org/10.4213/rm17

Использование Общероссийского математического портала Math-Net.Ru подразумевает, что вы прочитали и согласны с пользовательским соглашением

http: //www . mathnet.ru/rus/agreement

Параметры загрузки:

IP : 54.209 .52 .79

26 апреля 2023 г., 07:48:23 


\title{
ТЕРМОДИНАМИЧЕСКИЙ ФОРМАЛИЗМ ДЛЯ СИМВОЛИЧЕСКИХ ЦЕПЕЙ МАРКОВА СО СЧЕТНЫМ ЧИСЛОМ СОСТОЯНИЙ
}

\author{
Б. М. ГУРЕВИЧ, С. В. САВЧЕНКО
}

\section{СОДЕРЖАНИЕ}

Введение

Глава 1. Вариационные принципы и классификация функций, определенных на марковском множестве

$\S 1$. Основные определения. Вариационный принцип для топологического давления

$\S 2$. Производящие функции. Неотрицательные матрицы .............. 16

$\S 3$. Критерии устойчивой положительности ........................ . . . 25

$\S 4$. Равновесные и гиббсовские меры. Вариационный принцип Гиббса . . 38

Глава 2. Два класса инвариантных мер, связанных с СЦМ. Метод суб-

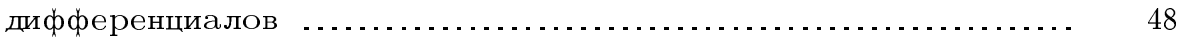

$\S 5$. Субдифференциал топологического давления ................... . . 48

§6. Асимптотически-равновесные меры. Сходимость равновесных мер, отвечающих конечным подцепям счетной СЦМ ................ 63

$\S 7$. Асимптотика дискретных инвариантных мер . . . . . . . . . . . . . . 73

Глава 3. Динамические дзета-ффункции, связанные с СЦМ ... . . . . . . . 81

$\S 8$. Теорема факторизации и некоторые ее следствия ............. . . 81

$\S 9$. Мероморфное продолжение дзета-функций и поведение дискретных

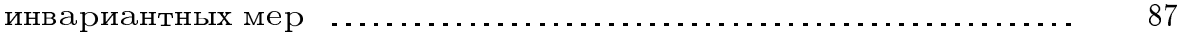

$\S 10$. Дзета-функция и спектральные свойства неотрицательных матриц . 94

Глава 4. Некоторые специальные классы СЦМ . . . . . . . . . . . . . . . . . . 98

$\S 11$. СЦМ с регулярным множеством периодических орбит .......... 98

$\S 12$. СЦМ с конечной областью прохождения циклов ................ . . 101

Список литературы ........................................ 105

Работа первого автора вьполнена при поддержке Фонда Сороса (грант № М8Х000) и Российского фонда фундаментальных исследований (грант № 96-01-00377).

Работа второго автора выполнена при поддержке Фонда Сороса (грант № М8Х000) и гранта INTAS-RFBR 95-418. 


\begin{abstract}
Введение
Символическая цепь Маркова (СЦМ) - это динамическая система, порожденная сдвигом на множестве последовательностей, которые можно отождествить с бесконечными путями конечного или счетного ориентированного графа. Обычно множество путей рассматривается вместе с заданной на нем естественной топологией, в которой сдвиг является непрерьвньм преобразованием. Отсюда - термин "топологическая цеп Маркова”, употребляемый гораздо чаше, чем СЦМ. Однако топология играет первостепенную роль далеко не во всех вопросах, касающихся этого объекта (примеры можно найти и в настоящей статье), поэтому эпитет "символическая" кажется нам в большей степени отвечаюшим существу дела. Впрочем, имеется еще английский термин "subshift of finite type", указывающий на то, что речь идет об ограничении сдвига на некоторое подмножество множества всех последовательностей с данньгм алфавитом, т.е. о "подсдвиге", причем это подмножество задается конечным числом ограничений (по крайней мере, в случае конечного алфавита). Строго говоря, subshift of finite type - несколько более общее понятие, чем СЦМ, однако первое сводится ко второму с помошњю простой перекодировки (см. ниже).
\end{abstract}

В теории динамических систем СЦМ играют двоякую роль: с одной стороны, они выступают в качестве простейших символических моделей некоторых классических динамических систем, в частности, систем гиперболического типа и рассеиваюших биллиардов (причем в последнем случае с неизбежностью возникают СЦМ с бесконечньм числом состояний) и служат одним из наиболее эффективных инструментов их изучения, с другой стороны, они достойны внимания и сами по себе, являясь источником разнообразных и достаточно содержательных математических задач.

Одно из основных направлений в исследовании СЦМ относится к так называемому термодинамическому формализму - кругу идей и понятий, тесно связанных со статистической физикой. Истоки этого направления можно усмотреть в работах середины 60-х годов, посвященных доказательству вариационного принципа для топологической энтропии непрерьвных преобразований компактных топологических пространств, но только в 70-е годы было осознано, что в классе символических динамических систем топологическая энтропия есть не что иное, как давление в решетчатой модели статистической физики с сингулярньм потенциалом, и появилась возможность рассматривать потенщиалы более общего вида, что привело к понятию топологического давления и изучению вероятностных мер на фазовом пространстве динамической системы (не только символической), которые можно интерпретировать как распределения Гиббса. Этому в значительной мере способствовали статьи Я. Г. Синая [1], Р. Боуэна [2] и Д. Рюэля [3], [4] (см. также [5]-[8]). Для СЦМ с конечным числом состояний топологическая энтропия и вариационные принципы рассматривались в работе В. Перри [9] еще до появления этих понятий в общем случае. В [10], [11] полученные им результаты были обобщены (насколько это оказалось возможньм) на СЦМ со счетным числом состояний. Выяснилось, что некомпактность фазового пространства приводит в этом случае к совершенно новьм задачам, не имеюшим смысла для СЦМ с конечным числом состояний.

Настоящая статья посвящена некоторым из этих задач. Она носит полуобзорный характер: доказательства, как правило, приводятся лишш в тех случаях, когда они отсутствуют в имеющейся литературе, новые результаты подробно доказываются, 
по ходу изложения формулируются нерешенные задачи. Для удобства читателя все выделенные формулировки (определения, леммы, теоремы и т. д.) внутри каждого параграфа включены в общий список и снабжены номерами вида N.1, N.2, .., где N - номер параграфо.

\section{Глава 1.}

\section{Вариационные принципы и}

\section{классификация функций, определенных на марковском множестве}

Обычно термодинамический формализм строится в ситуации, когда заданы преобразование $T$ некоторого пространства $X$ и функция $f$, определенная на этом пространстве. В этой статье роль $(X, T)$ будет, как правило, играть СЦМ со счетным числом состояний, определяемая некоторым ориентированным графом $G$, а на $f$ будут накладьваться те или иные ограничения. Поэтому во многих встречающихся ниже обозначениях присутствует пара $(G, f)$ или заменяюшие ее символы. Впрочем, иногда мы будем опускать символ $G$, если графф явным образом фиксирован.

\section{$\S$ 1. Основные определения. Вариационный принцип для топологического давления}

Пусть $G$ - ориентированньй графи с конечньм или счетным множеством вершин $V=V(G)$ и множеством ребер $E=E(G) \subset V \times V$.

1.1. ОПРЕДЕЛЕНИЕ. Назовем последовательность $\gamma=\left(v_{0}, v_{1}, \ldots, v_{n}\right)$, где $n \geqslant 1$ и $v_{i} \in V$ при $0 \leqslant i \leqslant n$, путем длины $l(\gamma)=n$ графа $G$ (или $G$-путем), если $\left(v_{i}, v_{i+1}\right) \in E$ при $0 \leqslant i \leqslant n-1$; каждое такое ребро $\left(v_{i}, v_{i+1}\right)$ будем назьвать принадлежашим $\gamma$, а сам путь $\gamma$ - проходящим через $\left(v_{i}, v_{i+1}\right)$. Будем говорить, что путь $\gamma$ выходит из $v \in V$, входит в $v$ и проходит через $v$, если соответственно $v_{0}=v$, $v_{n}=v$ и $v_{i}=v$ при некотором $i \neq 0, n$. Если при этом $v_{n}=v_{0}$, будем называть $\gamma$ ииклом (или, более точно, $v$-ииклом); $v$-циклы, в которых $v_{i} \neq v$ при всех $i \neq 0, n$, назовем простым м. Все циклы графра $G$ будем назьвать $G$-ииклами. Путь, выходящий из $v$ и входящий в $v^{\prime}$, будем назьвать ведущим из $v$ в $v^{\prime}$, а путь, проходяший через какую-либо вершину $w \in W$, где $W \subset V,-$ проходяшим через $W$. Если $\gamma=\left(v_{0}, v_{1}, \ldots, v_{n}\right), \gamma^{\prime}=\left(v_{0}^{\prime}, v_{1}^{\prime}, \ldots, v_{m}^{\prime}\right)$ - два $G$-пути, причем $v_{0}^{\prime}=v_{n}$, то последовательность $\gamma \gamma^{\prime}=\left(v_{0}, v_{1}, \ldots, v_{n}, v_{1}^{\prime}, \ldots, v_{m}^{\prime}\right)$, также являющуюся $G$-путем (длина которого, очевидно, равна $m+n)$, будем назьвать произведением путей $\gamma$ и $\gamma^{\prime}$. Множество всех $G$-путей обозначим через $\Gamma(G)$.

Ясно, что всякий $v$-цикл представим, притом единственным способом, в виде произведения простых $v$-циклов.

В пространстве последовательностей $X=V^{\mathbb{Z}}$ выделим подмножество $X(G)$, состоящее из тех $x=\left(x_{i}, i \in \mathbb{Z}\right)$, для которых $\left(x_{i}, x_{i+1}\right) \in E(G)$ при всех $i \in \mathbb{Z}$. Последовательности, входяшие в $X(G)$, будем назьвать двусторонне-бесконечными $G$-nyтями. На $X$ определен сдвиг $S$, переводящий последовательность $x=\left(x_{i}, i \in \mathbb{Z}\right)$ в последовательность $x^{\prime}=\left(x_{i}^{\prime}, i \in \mathbb{Z}\right)$, где $x_{i}^{\prime}=x_{i+1}$. Очевидно, множество $X(G)$ является $S$-инвариантным. Будем назьвать его марковским множеством. 
1.2. ОПРЕДЕЛЕНИЕ. Пара $(X(G), S)$ назьвается символической цепью Маркова (СЦМ), отвечаюшей графу $G$.

Поскольку преобразование $S$ всегда одно и то же, СЦМ $(X(G), S)$ можно отождествить с множеством $X(G)$, называемым марковским множеством, или даже с самим графом $G$. По определению $X(G)$ состоит из последовательностей, в которых каждая пара стоящих рядом символов “допустима" - служит ребром графа $G$.

Можно обобшить это определение, если задать при каком-нибудь $n \geqslant 2$ произвольную совокупность $\mathscr{A}$ последовательностей длины $n+1$ и, назвав их допустимыми, рассмотреть множество $X(\mathscr{A})$ тех бесконечных последовательностей $x \in X$, в которых каждьй отрезок длины $n+1$ является допустимым. Множество $X(\mathscr{A})$, как и $X(G)$, инвариантно относительно $S$, и пару $(X(\mathscr{A}), S)$ естественно назвать СЦМ порядка $n$. В таком случае $(X(G), S)$ будет СЦМ первого порядка. Очевидно, всякая СЦМ порядка 1 есть одновременно и СЦМ порядка $n$ при любом $n>1$. В зарубежной математической литературе по отношению к СЦМ любого (конечного) порядка часто применяется термин subshift of finite type, происхождение которого было объяснено во введении. Мы не будем рассматривать СЦМ порядка большего единицы: как уже было сказано, их можно свести к СЦМ первого порядка. Делается это следующим образом.

При $n \geqslant 1$ введем отображение $\Phi_{n}$, которое каждой последовательности $x=\left(x_{i}, i \in\right.$ $\mathbb{Z}), x_{i} \in V$, ставит в соответствие последовательность $y=\left(y_{i}, i \in \mathbb{Z}\right)$, где $y_{i}=$ $\left(x_{i}, x_{i+1}, \ldots, x_{i+n-1}\right)$. Очевидно, $\Phi_{n}$ обратимо на $X$ и коммутирует со сдвигом, а $\Phi_{1}$ - тождественное преобразование. Если $\mathscr{A}$ - произвольное множество последовательностей длины $n+1$, то, как легко видеть, $\Phi_{n} X(\mathscr{A})$ - марковское множество. Оно определяется графом $G_{n}(\mathscr{A})$ с множеством вершин, состояшим из тех последовательностей $\left(v_{i_{1}}, \ldots, v_{i_{n}}\right) \in V^{n}$, которые можно продолжить до последовательности из $\mathscr{A}$ приписыванием одного символа слева или справа, и множеством ребер, состояшим из таких пар $\left(\left(v_{i_{1}}, \ldots, v_{i_{n}}\right),\left(v_{i_{1}}^{\prime}, \ldots, v_{i_{n}}^{\prime}\right)\right)$, для которых $v_{i_{k}}^{\prime}=v_{i_{k+1}}$ при $k=1, \ldots, n-1$ и $\left(v_{i_{1}}, \ldots, v_{i_{n}}, v_{i_{n}}^{\prime}\right) \in \mathscr{A}$. Будем назьвать $\Phi_{n}$ редукцией порядка $n$ или $n$-редукиией. В частном случае, когда $X(\mathscr{A})$ само является марковским множеством, т.е. $X(\mathscr{A})=X(G)$, мы можем рассматривать $G_{n}(\mathscr{A})$ как образ графа $G$ при индуцированном отображении граффов, которое в дальнейшем будем обозначать тем же символом $\Phi_{n}$. Пользуясь тем, что $\Phi_{n}$ обратимо на $X(G)$ и $\Phi_{n} X(G)=X\left(\Phi_{n} G\right)$, можно поставить в соответствие каждой функции $f: X(G) \rightarrow \mathbb{R}$ функцию $f_{n}=\Phi_{n}^{*} f$, где $\Phi_{n}^{*} f(y)=f\left(\Phi_{n}^{-1} y\right), y \in X\left(G_{n}\right)$ (использование одного и того же обозначения для индуцируюших друг друга отображений разных множеств не должно вызьвать недоразумений).

Снабдим множество $V$ дискретной топологией и зададим на $X$ топологию прямого произведения. Очевидно, конечномерные цилиндры, т.е. множества вида $\{x \in X$ : $\left.x_{i}=v_{n_{i}}, i=1, \ldots, k\right\}$ образуют базу этой топологии, множество $X(G)$ в ней замкнуто, а сдвиг $S$ и $n$-редукция $\Phi_{n}$ при любом $n$ являются гомеоморфизмами. Ту же топологию на $X$ и, следовательно, ту же индуцированную топологию на $X(G)$ можно получить с помошью метрики. Пусть $\theta \in(0,1)$ и $\rho$-метрика на $V$, удовлетворяюшая условию $\sup _{v, v^{\prime} \in V} \rho\left(v, v^{\prime}\right)<\infty$. Для любых $x=\left(x_{i}, i \in \mathbb{Z}\right), x^{\prime}=\left(x_{i}^{\prime}, i \in \mathbb{Z}\right)$ положим

$$
d_{\rho, \theta}\left(x, x^{\prime}\right)=\sum_{i \in \mathbb{Z}} \theta^{|n|} \rho\left(x_{i}, x_{i}^{\prime}\right) .
$$


Легко проверить, что $d_{\rho, \theta}-$ метрика на $X$. Для нас будут важны метрики $\rho$ двух типов, а именно, метрики, удовлетворяющие при какой-нибудь нумерации вершин условию

$$
\inf _{i, j: i \neq j} \rho\left(v_{i}, v_{j}\right)>0
$$

и условию

$$
\lim _{n \rightarrow \infty} \sup _{i, j \geqslant n} \rho\left(v_{i}, v_{j}\right)=0
$$

(очевидно, при любой другой нумерации было бы то же самое). Число $\theta$ и конкретньй вид метрики каждого типа в дальнейшем не играют существенной роли, поэтому впредь будем считать, что $\theta=1 / 2, \rho_{1}\left(v_{i}, v_{j}\right) \equiv 1$ и $\rho_{2}\left(v_{i}, v_{j}\right) \equiv\left|i^{-1}-j^{-1}\right|$.

Напомним определение топологического давления $P(Y, T, f)$ для непрерьвного отображения $T: Y \rightarrow Y$ компактного топологического пространства $Y$ и функции $f \in$ $C(Y, \mathbb{R})$ (подробности см. в [6], [7]). Пусть $\mathscr{U}$ - конечное покрытие пространства $Y$ открытыми множествами $U_{i}$ и $\mathscr{U}^{(m)}, m=0,1, \ldots,-$ покрытие, образованное множествами вида

$$
U_{i_{0}, \ldots, i_{m-1}}=\bigcap_{k=0}^{m-1} T^{-k} U_{i_{k}} .
$$

Для каждого непустого элемента $W$ покрытия $\mathscr{U}^{(m)}$ положим

$$
f^{(m)}(W)=\sup _{y \in W} \exp \left[\sum_{k=0}^{m-1} f\left(T^{k} y\right)\right] .
$$

Пусть

$$
Z_{m}(\mathscr{U})=\min _{\mathscr{U}^{\prime}} \sum_{W \in \mathscr{U}^{\prime}} f^{(m)}(W)
$$

где минимум берется по всем подпокрытиям $\mathscr{U}^{\prime}$ покрытия $\mathscr{U}$. Тогда сушествует предел

$$
P(Y, T, f, \mathscr{U})=\lim _{m \rightarrow \infty} m^{-1} \log Z_{m}(\mathscr{U}) .
$$

Топологическое давление $P(Y, T, f)$ определяется равенством

$$
P(Y, T, f)=\sup _{\mathscr{U}} P(Y, T, f, \mathscr{U})
$$

В частном случае, когда $f \equiv 0$, мы приходим к определению топологической энтроnuu $h(Y, T)=P(Y, T, 0)$. 
1.3. ЗАмЕчАниЕ. Ниже будут встречаться ситуации, когда пространство $Y$ вложено в некоторое большее пространство $X$, а функция $f$ определена на $X$. Строго говоря, в таких случаях следовало бы вместо $P(Y, T, f)$ писать $P\left(Y, T,\left.f\right|_{Y}\right)$. Мы, однако, этого делать не будем, поскольку $Y$ и так присутствует в обозначениях в качестве первого аргумента функции $P$. Точно так же будем поступать и с некоторыми другими обозначениями, аналогичньми этому.

Обозначим через $\mathscr{M}(Y, T)$ совокупность $T$-инвариантных вероятностных борелевских мер на $Y$. Равенство

$$
P(Y, T, f)=\sup _{\mu \in \mathscr{M}(Y, T)}\left[h_{\mu}(T)+\int f d \mu\right]
$$

носит название вариационного принципа для топологического давления. В приведенной здесь форме оно было доказано П. Уолтерсом [6] (более ранние результаты относились к топологической энтропии, первое обобщение на случай $f \not \equiv 0$ получено Рюэлем [3]).

Меры $\mu$, для которых в правой части (1.5) достигается верхняя грань, назьваются равновесныци мерами (более точно, $f$-равновесныцми), а также равновесными распределениями и равновесными состояниями.

Я. Б. Песин и Б. С. Пищкель [12] обобшили понятие топологического давления на некомпактный случай (для топологической энтропии это ранее сделал Боуэн [13], [14]) в предположении, что $Y$ - подмножество компактного метрического пространства $X$, а функцию $f$ можно продолжить по непрерьвности на $X$. Приведем их определение.

Пусть $X$-компактное метрическое пространство, $Y \subset X, T: Y \rightarrow Y$-непрерьвное отображение и $f: X \rightarrow \mathbb{R}$ - непрерьвная функция. Для любого конечного открытого покрытия $\mathscr{U}=\left(U_{1}, \ldots, U_{k}\right)$ пространства $X$, любого $n \in \mathbb{Z}^{+}$и любого набора $I=\left(i_{0}, \ldots, i_{n-1}\right) \in\{1, \ldots, k\}^{n}$ положим

$$
\begin{gathered}
l(I)=n, \quad U(I)=\bigcap_{s=0}^{n-1} T^{-s} U_{i_{s}}, \\
F(I)= \begin{cases}\sup _{x \in U(I)} \sum_{s=0}^{n-1} f\left(T^{s} x\right), & U(I) \neq \varnothing, \\
-\infty, & U(I)=\varnothing .\end{cases}
\end{gathered}
$$

Пусть $Z \subset Y, N \geqslant 1, \mathscr{J}_{N}=\bigcup_{n \geqslant N}\{1, \ldots, k\}^{n}$ и $\mathscr{J}_{N}(Z, T, \mathscr{U})$ - совокупность тех $\mathscr{J}^{\prime} \subset \mathscr{J}_{N}$, для которых $\bigcup_{I \in \mathscr{F}^{\prime}} U(I) \supset Z$. Для любого $\lambda \in \mathbb{R}$ положим

$$
M_{X}(Z, T, f, \mathscr{U}, \lambda, N)=\inf _{\mathscr{J}^{\prime} \in \mathscr{F}_{N}(Z, T, \mathscr{U})} \sum_{I \in \mathscr{F}^{\prime}} \exp [-\lambda l(I)+F(I)] .
$$

Так как $M_{X}(Z, T, f, \mathscr{U}, \lambda, N)$, очевидно, не убьвает по $N$, существует предел

$$
m_{X}(Z, T, f, \mathscr{U}, \lambda)=\lim _{N \rightarrow \infty} M_{X}(Z, T, f, \mathscr{U}, \lambda, N),
$$

причем возможен лишь один из двух случаев: либо $m_{X}(Z, T, f, \mathscr{U}, \lambda)=0$ при всех $\lambda \in \mathbb{R}$, либо найдется такое $\lambda_{0} \in \mathbb{R}$, что $m_{X}(Z, T, f, \mathscr{U}, \lambda)=\infty$, если $\lambda<\lambda_{0}$, и 
$m_{X}(Z, T, f, \mathscr{U}, \lambda)=0$, если $\lambda>\lambda_{0}$. Положим $P_{X}(Z, T, f, \mathscr{U})$ равным нулю в первом случае и равным $\lambda$ - во втором. Пусть, наконец,

$$
P_{X, d}(Z, T, f)=\lim _{\operatorname{diam}(\mathscr{U}) \rightarrow 0} P_{X}(Z, T, f, \mathscr{U}),
$$

где $\operatorname{diam}(\mathscr{U})$ - наибольший из диаметров множеств $U_{i}$ (сушествование предела доказывается весьма просто).

1.4. ЗАмЕчАниЕ. В [12] имеется вариант определения топологического давления для $f \equiv 0$ (т.е. топологической энтропии), когда $T$ - непрерывное преобразование произвольного топологического пространства $Y$ (а объемлющее пространство $X$ отсутствует). Это определение отличается от только что приведенного лишш тем, что в нем $\mathscr{U}$ - конечное открытое покрытие пространства $Y$ (а не $X$ ) и в (1.6) вместо предела берется точная верхняя грань по всем таким $\mathscr{U}$. Обозначив полученную величину через $h_{Y}(Z, T)$, положим $h(Y, T)=h_{Y}(Y, T)$. В [12] показано, что $h(Y, T)$ совпадает с энтропией, введенной Боуэном [13].

Топологическое давление $P_{X, d}(Z, T, f)$ обладает следуюшими свойствами:

а) если множество $Y$ компактно, то

$$
P_{X, d}(Y, T, f)=P(Y, T, f)
$$

(см. (1.5) и замечание 1.3);

б) если $Z_{1} \subseteq Z_{2} \subseteq Y$, то

$$
P_{X, d}\left(Z_{1}, T, f\right) \leqslant P_{X, d}\left(Z_{2}, T, f\right)
$$

в) справедливо неравенство

$$
P_{X, d}(Y, T, f) \geqslant \sup _{\mu \in \mathscr{M}(Y, T)}\left[h_{\mu}(T)+\int f d \mu\right] .
$$

Говорят, что $P_{X, d}(Y, T, f)$ удовлетворяет вариационному принципу, если в (1.9) имеет место равенство. Это всегда так в случае компактного $Y$ (см. [6]).

Рассмотрим теперь более общую ситуацию, когда заданы топологическое пространство $Y$, непрерьвное отображение $T: Y \rightarrow Y$ и непрерьвная функция $f: Y \rightarrow \mathbb{R}$. Положим

$$
P^{\text {int }}(Y, T, f)=\sup _{K \in \mathscr{K}(Y, T)} P(K, T, f)
$$

где $\mathscr{K}(Y, T)$ - совокупность $T$-инвариантных компактных множеств $K \subset Y$ (если $\mathscr{K}(Y, T)=\varnothing$, полагаем $\left.P^{\operatorname{int}}(Y, T, f)=-\infty\right)$. Очевидно, $P^{\operatorname{int}}(Y, T, f)$ - топологический инвариант: если $\left(Y^{\prime}, T^{\prime}, f^{\prime}\right)$ - другая тройка того же типа, что $(Y, T, f)$, и сушествует такой гомеоморфизм $\Phi: Y \rightarrow Y^{\prime}$, что $T^{\prime} \Phi y=\Phi T y, f^{\prime}(\Phi y)=f(y)$ при всех $y \in Y$, то $P^{\text {int }}\left(Y^{\prime}, T^{\prime}, f^{\prime}\right)=P^{\text {int }}(Y, T, f)$.

В предположении, что $Y$ можно непрерывно вложить в компактное метрическое пространство $(X, d)$, а функцию $f$ - продолжить по непрерьвности на $X$, положим

$$
P^{\operatorname{ext}}(Y, T, f)=\inf _{(X, d)} P_{X, d}(Y, T, f)
$$


где inf берется по всем упомянутьм вложениям.

Очевидно, $P^{\mathrm{int}}$ и $P^{\mathrm{ext}}$ определяются уже только одной топологической структурой пространства $Y$ (причем $P^{\text {int }}$ имеет смысл и в том случае, когда функция $f$ неограничена). Для ограниченной $f$ справедливость вариационного принципа в компактном случае приводит к неравенству

$$
P^{\mathrm{int}}(Y, T, f) \leqslant \sup _{\mu \in \mathscr{M}(Y, T)}\left[h_{\mu}(T)+\int f d \mu\right],
$$

а (1.6) - к неравенству

$$
P^{\operatorname{ext}}(Y, T, f) \geqslant \sup _{\mu \in \mathscr{M}(Y, T)}\left[h_{\mu}(T)+\int f d \mu\right] .
$$

Если в (1.10) и (1.11) имеет место равенство, будем, аналогично предыдущему, говорить о справедливости вариационного принципа для $P^{\text {int }}$ и $P^{\text {ext }}$ соответственно.

Из (1.10), (1.11) видно, что

$$
P^{\mathrm{int}}(Y, T, f) \leqslant P^{\mathrm{ext}}(Y, T, f)
$$

во всех случаях, когда правая часть (1.12) имеет смысл. Разумеется, в компактном случае $P^{\text {int }}$ и $P^{\text {ext }}$ совпадают. Если же $Y$ некомпактно, то в (1.10) и, следовательно, в (1.12) может быть строгое неравенство. Чтобы убедиться в этом, достаточно взять в качестве $T$ какой-нибудь минимальный гомеоморфизм компактного пространства $X$, имеющий положительную топологическую энтропию, в качестве $Y$ - множество $X \backslash \mathscr{O}(x)$, где $\mathscr{O}(x)$ - орбита произвольной точки $x \in X$, и положить $f \equiv 0$. Очевидно, левая часть (1.10) в этом случае будет равна нулю, а правая - топологической энтропии преобразования $T$.

Из сказанного следует, что $P^{\text {int }}$ может удовлетворять вариационному принципу лишь при наличии у $Y$ достаточно богатого запаса $T$-инвариантных компактных подмножеств. Было бы интересно также найти обшие условия, при которых справедлив вариационный принцип для $P^{\mathrm{ext}}$.

В случае СМЦ ситуация значительно яснее, чем в общем случае. Заметим, прежде всего, что для любой метрики $\rho$ на $V$ и любого $\theta$ преобразование $S$ равномерно непрерьвно в метрике $d=d_{\rho, \theta}$ (см. (1.1)). Поэтому $S$ можно продолжить по непрерьвности до преобразования $S_{d}: Y_{d} \rightarrow Y_{d}$, где $Y_{d}$ - пополнение пространства $Y=X(G)$ по метрике $d$. Если функция $f$, заданная на $Y$, также равномерно непрерьвна, то и ее можно продолжить до непрерьвной функции $f_{d}$ на $Y_{d}$.

Проведем теперь операцию противоположного характера: для произвольного конечного связного подграфа $G_{0}$ графа $G$ ограничим как сдвиг $S$, так и функцию $f$ на множество $X\left(G_{0}\right) \subset X(G)$, которое, очевидно, $S$-инвариантно и компактно.

Tеорема ([15], см. также замечание 5.15). Если $\rho$ - метрика на $V$, удовлетворяющая условию (1.3), $d=d_{\rho, \theta}-$ индуцированная ею метрика на $X(G)$ и $X_{d}(G)$ - пополнение пространства $(X(G), d)$, то для любого $\theta \in(0,1)$

(a) пространство $X_{d}(G)$ компактно; 
(б) для любой функции $f: X(G) \rightarrow \mathbb{R}$, ограниченной и равномерно непрерьвной в метрике $d$, выполняется соотношение

$$
\sup _{G_{0}} P\left(X\left(G_{0}\right), S, f\right)=P\left(X_{d}(G), S, f_{d}\right),
$$

где $\operatorname{sup~берется~по~всем~конечным~связным~подграфам~} G_{0}$ графа $G$.

1.6. ЗАмЕчАниЕ. В [15] эта теорема доказана для конкретного выбора $\rho$ и $\theta$ (а именно, для $\rho\left(v_{i}, v_{j}\right)=\left|i^{-1}-j^{-1}\right|$ при некоторой нумерации вершин и для $\theta=1 / 2$ ), однако приведенное там доказательство годится и для общего случая.

1.7. СлЕДСТВИЕ. Если функиия $f$ равномерно непрерывна в метрике $d=d_{\rho, \theta}$, где $\rho$ удовлетворяет условию (1.3), то

$$
\begin{aligned}
& P^{\text {int }}(X(G), S, f)=P^{\operatorname{ext}}(X(G), S, f), \\
& P^{\operatorname{ext}}(X(G), S, f)=P_{X_{d}(G), d}(X(G), S, f)
\end{aligned}
$$

и для $P^{\mathrm{int}}, P^{\mathrm{ext}}$ выполняется вариачионный принцип.

ДокАЗАТЕЛЬСТво. Для любого множества $Y \subset X(G)$ положим

$V(Y)=\left\{v \in V(G):\right.$ сушествуют такие $x=\left(x_{i}, i \in \mathbb{Z}\right) \in Y$ и $n \in \mathbb{Z}$, что $\left.x_{n}=v\right\}$.

Пусть $Y$ инвариантно относительно $S$; тогда

$$
V(Y)=\left\{v \in V(G): \text { сушествует такое } x=\left(x_{i}, i \in \mathbb{Z}\right) \in Y, \text { что } x_{0}=v\right\} .
$$

Если, кроме того, $Y$ компактно, то $V(Y)$ конечно. Так как граф $G$ связен, он обладает связным подграфом $G_{0}$, вершины которого образуют конечное множество $V_{0} \subset V$, содержащее $V(Y)$, а ребрами служат те ребра графа $G$, у которых начало и конец принадлежат $V_{0}$ (это, в частности, следует из леммы 3.10 , доказываемой ниже). Легко видеть, что $Y \subseteq X\left(G_{0}\right)$ и, следовательно,

$$
P(Y, S, f) \leqslant P\left(X\left(G_{0}\right), S, f\right) .
$$

Тем самьм,

$$
P^{\text {int }}(X(G), S, f)=\sup _{G_{0} \in \mathscr{G}_{0}(G)} P\left(X\left(G_{0}\right), S, f\right)
$$

где $\mathscr{G}_{0}(G)$ - множество всех конечных подграфов графа $G$. Отсюда в силу теоремы 1.5 получаем

$$
P^{\text {int }}(X(G), S, f)=P\left(X_{d}(G), S_{d}, f_{d}\right) .
$$

С другой стороны, $(1.8),(1.7)$ и определение $P(X(G), S, f)$ дают

$$
\begin{aligned}
P\left(X_{d}(G), S_{d}, f_{d}\right) & =P_{X_{d}(G), d}\left(X_{d}(G), S_{d}, f_{d}\right) \\
& \geqslant P_{X_{d}(G), d}(X(G), S, f) \geqslant P^{\operatorname{ext}}(X(G), S, f) .
\end{aligned}
$$

Сравнивая (1.16), (1.17) с (1.12), приходим к (1.13), (1.14).

Что касается вариационного принципа для $P=P^{\mathrm{ext}}$, то он моментально следует из $(1.13),(1.11)$ и справедливости вариационного принципа для $P\left(X\left(G_{0}\right), S, f\right)$ при любом конечном $G_{0}$. Следствие доказано. 
1.8. ЗАмЕчАнИЕ. Очевидно, равенство (1.15) справедливо для любой непрерьвной функции, определенной на $X(G)$, даже неограниченной. Заметим, что если метрика $\rho_{1}$ на $V$ удовлетворяет условию (1.2), а метрика $\rho_{2}-$ условию $(1.3)$ и если $d_{i}=d_{\theta, \rho_{i}}$, $i=1,2,-$ соответствуюшие метрики на $X(G)$, то всякая $d_{2}$-равномерно непрерывная функция является и $d_{1}$-равномерно непрерьвной, но, вообще говоря, не наоборот. Поэтому в следствии 1.7 нельзя заменить метрику $d_{2}$ на метрику $d_{1}$ (даже в случае $f \equiv 0)$. Тем не менее справедливо следуюшее утверждение.

1.9. ТЕОРемА. Если функция $f$ ограничена и равномерно непрерывна в метрике $d_{1}$, то для $P^{\operatorname{int}}(X(G), S, f)$ выполняется вариачионный принцип.

Для доказательства нам потребуется ввести один класс функций, которьй будет использоваться и в дальнейшем.

1.10. ОПРЕДЕлЕнИЕ. Для любых $k, l \in \mathbb{Z}$ обозначим через $\mathscr{F}_{k}^{l}(G)$ совокупность действительных функций, постоянных на цилиндрических множествах с носителем $[k, l]:$

$$
\begin{array}{r}
\mathscr{F}_{k}^{l}(G)=\left\{f: f(x)=f\left(x^{\prime}\right), \text { если } x=\left(x_{i}, i \in \mathbb{Z}\right), x^{\prime}=\left(x_{i}^{\prime}, i \in \mathbb{Z}\right)\right. \\
\text { и } \left.x_{i}=x_{i}^{\prime} \text { при } k \leqslant i \leqslant l\right\} .
\end{array}
$$

Такие функции будем назьвать локальными

Легко видеть, что все локальные функции непрерывны.

ДоКАЗАТЕЛЬСТво теОРемы 1.9. Начнем со случая, когда

$$
\sup _{\mu \in \mathscr{M}(X(G), S)}\left[h_{\mu}(S)+\int f d \mu\right]=\infty .
$$

Так как функция $f$ ограничена, (1.18) эквивалентно равенству

$$
\sup _{\mu \in \mathscr{M}(X(G), S)} h_{\mu}(S)=\infty
$$

а так как в силу следствия 1.7 для $P^{\mathrm{int}}(X(G), S, 0)$ выполняется вариационньй принцип, (1.19) означает, что $P^{\operatorname{int}}(X(G), S, 0)=\infty$. Для $P^{\text {int }}\left(X\left(G_{0}\right), S, 0\right)$ при $G_{0} \in \mathscr{G}_{0}(G)$ также вьполняется вариационньй принцип. Поэтому (см. (1.15) и замечание 1.8$)$

$$
\sup _{G_{0} \in \mathscr{G}_{0}(G)} P^{\text {int }}\left(X\left(G_{0}\right), S, 0\right)=\sup _{G_{0} \in \mathscr{G}_{0}(G)} \sup _{\mu \in \mathscr{M}\left(X\left(G_{0}\right), S\right)} h_{\mu}(S)=\infty
$$

Но тогда

$$
\begin{aligned}
P^{\text {int }}(X(G), S, f) & =\sup _{G_{0} \in \mathscr{G}_{0}(G)} P^{\text {int }}\left(X\left(G_{0}\right), S, f\right) \\
& =\sup _{G_{0} \in \mathscr{G}_{0}(G)} \sup _{\mu \in \mathscr{M}\left(X\left(G_{0}\right), S\right)}\left[h_{\mu}(S)+\int f d \mu\right]=\infty .
\end{aligned}
$$


Предположим теперь, что левая часть равенства (1.18) конечна и, следовательно, $P^{\text {int }}(X(G), S, f)<\infty$. Пользуясь равномерной непрерьвностью $f$, подберем такую последовательность функций $f_{n} \in \mathscr{F}_{-n}^{n}(G)$, что

$$
\lim _{n \rightarrow \infty}\left\|f-f_{n}\right\|=0
$$

где $\left\|f-f_{n}\right\|=\sup _{x \in X(G)}\left|f(x)-f_{n}(x)\right|$. Очевидно,

$$
\sup _{\mu \in \mathscr{M}(X(G), S)}\left[h_{\mu}(S)+\int f d \mu\right]-\sup _{\mu \in \mathscr{M}(X(G), S)}\left[h_{\mu}(S)+\int f_{n} d \mu\right] \mid \leqslant\left\|f-f_{n}\right\|
$$

(последнее неравенство вытекает из соотношения (1.15) и вариационного принципа для $P^{\operatorname{int}}\left(X\left(G_{0}\right), S, g\right)$, где $G_{0} \in \mathscr{G}_{0}(G)$ и $g$ - непрерьвная функщия на $\left.X(G)\right)$. Отсюда следует, что достаточно доказать вариационный принщип для $P^{\operatorname{int}}(X(G), S, f)$ в предположении, что $f$ - ограниченная функция из $\mathscr{F}_{-n}^{n}(G), n=1,2, \ldots$ Так как обе части равенства, выражаюшего вариационный принцип, не меняются при замене $f(x)$ на $f(S x)$, достаточно ограничиться случаем, когда $f \in \mathscr{F}_{0}^{n}(G), n=1,2, \ldots$ Кроме того, можно считать, что $n=1$. В самом деле, если $f \in \mathscr{F}_{0}^{n}(G), n \geqslant 2$, применим к $X(G)$ преобразование $\Phi_{n}$ (определенное вьше). Напомним, что $\Phi_{n} X(G)=X\left(G_{n}\right)$, где $G_{n}$ - граф, вершинами которого служат $G$-пути длины $n-1$, а ребрами - пары $G$-путей $\left(\gamma^{\prime}, \gamma^{\prime \prime}\right)$, удовлетворяюшие условию: найдется $G$-путь длины $n$, начало которого совпадает с $\gamma^{\prime}$, а конец - с $\gamma^{\prime \prime}$. Пользуясь тем, что $\Phi_{n}$ обратимо, положим, как и раньше, $f_{n}(y)=\left(\Phi_{n} f\right)(y)=f\left(\Phi_{n}^{-1} y\right), y \in X\left(G_{n}\right)$. Очевидно, $f_{n}$ - ограниченная непрерьвная функция из $\mathscr{F}_{0}^{1}\left(G_{n}\right)$ и

$$
\begin{aligned}
P^{\text {int }}(X(G), S, f) & =P^{\text {int }}\left(X\left(G_{n}\right), S, f_{n}\right), \\
\sup _{\mu \in M(X(G), S)}\left[h_{\mu}(S)+\int f d \mu\right] & =\sup _{\mu \in \mathscr{M}\left(X\left(G_{n}\right), S\right)}\left[h_{\mu}(S)+\int f_{n} d \mu\right] .
\end{aligned}
$$

Таким образом, вариационньй принцип для $P^{\mathrm{int}}(X(G), S, f)$ достаточно доказать в случае ограниченной непрерьвной функции $f \in \mathscr{F}_{0}^{1}(G)$, понимая под $G$ произвольный связный ориентированный граф со счетным множеством вершин. Но такой результат имеется в [16], [25] (см. также $\S 4$ настояшей статьи). Тем самьм теорема 1.9 доказана.

Остановимся теперь на частном случае, когда $f \equiv 0$, т.е. на случае топологической энтропии. Разумеется, к нему применимо следствие 1.7. Однако известны еще по крайней мере три определения топологической энтропии непрерьвного отображения, которые, вообще говоря, не эквивалентны в некомпактном случае, и представляется естественным вопрос, к чему они приводят в случае СЦМ. Два из этих определений, содержащиеся соответственно в [13] и [12], дают одну и ту же величину (см. замечание 1.4), третье (а хронологически - первое) предложено Боуэном [14] и относится к непрерьвным преобразованиям метрических пространств. Оно состоит в следующем.

Пусть $(X, d)$ - метрическое пространство и $T: X \rightarrow X$-непрерьвное отображение. Множество $D \subset X$ назьвается $(n, \varepsilon)$-плотньм в множестве $F \subset X\left(n \in \mathbb{Z}^{+}, \varepsilon>0\right)$, 
если для всякой точки $x \in F$ найдется такая точка $y \in D$, что $d\left(T^{i} x, T^{i} y\right) \leqslant \varepsilon$ при $i=0, \ldots, n-1$. Для любого компактного $K \subset X$ положим

$$
\begin{gathered}
r_{n}(X, d, T, K, \varepsilon)=\inf \{\# D: D \text { является }(n, \varepsilon) \text {-плотным в } K\} \\
h(X, d, T, K, \varepsilon)=\varlimsup_{n \rightarrow \infty} n^{-1} \ln r_{n}(X, d, T, K, \varepsilon) \\
h(X, d, T, K)=\lim _{\varepsilon \rightarrow 0} h(X, d, T, K, \varepsilon)
\end{gathered}
$$

(очевидно, $h(X, d, T, K, \varepsilon)$ не убывает по $\varepsilon$ ). Топологическая энтропия $h(X, d, T)$ определяется равенством

$$
h(X, d, T)=\sup \{h(X, d, T, K): K \subset X \text { компактно }\} .
$$

Подставим в это определение вместо $d$ введенные выше метрики $d_{1}$ и $d_{2}$ (см. (1.1) и замечание 1.6).

1.11. ТеОРемА. Пусть $G$ - связный ориентированный граф с конечным или счетным множеством вершин $V$. Тогда

$$
\begin{aligned}
& h\left(X(G), d_{2}, S\right)=P^{\operatorname{int}}(X(G), S, 0), \\
& h\left(X(G), d_{1}, S\right)=\sup _{v \in V} \varlimsup_{n \rightarrow \infty} \frac{1}{n} \ln \# \Gamma^{+}(v, n),
\end{aligned}
$$

где $\Gamma^{+}(v, n)$ - множсество $G$-путей длинь $n$, выходящих из вершинь $v \in V$.

ДокАЗАТЕльство. Равенство (1.24) доказано в [17] (см. предложение 1.3 из указанной статьи); там же (теорема 1.1) доказано равенство (1.25) в предположении, что для каждой вершины $v \in V$ множество входящих в нее и выходящих из нее ребер конечно (графы с таким свойством называются локально-конечными). Более широкий класс образуют графы, по отношению к которьм условие конечности накладьвается лишь на выходящие ребра (такие графы будем называть локально-конечными вперед). Докажем для них сначала неравенство

$$
h\left(X(G), d_{1}, S\right) \leqslant \sup _{v \in V} \varlimsup_{n \rightarrow \infty} \frac{1}{n} \ln \# \Gamma^{+}(v, n) .
$$

Для любого множества $F \subset X(G)$ и любого $n \in \mathbb{Z}$ положим

$$
V(F, n)=\left\{v \in V: \text { сушествует такое } x=\left(x_{i}, i \in \mathbb{Z}\right) \in F, \text { что } x_{n}=v\right\} \text {. }
$$

Очевидно, компактность $F$ равносильна конечности всех $V(F, n), n \in \mathbb{Z}$. Фиксируем произвольное $\varepsilon>0$ и по заданным $\rho(\cdot, \cdot)$ и $\theta$ (входяшим в определение метрики $d_{1}$, см. замечание 1.8$)$ подберем такое $k=k(\varepsilon)$, что $d_{1}\left(x, x^{\prime}\right) \leqslant \varepsilon$ для любых $x=\left(x_{i}, i \in \mathbb{Z}\right), x^{\prime}=\left(x_{i}^{\prime}, i \in \mathbb{Z}\right)$, удовлетворяюших условию $x_{i}=x_{i}^{\prime}$ при $|i| \leqslant k$. Возьмем любое компактное множество $K$ и при $n \in \mathbb{Z}^{+}$поставим в соответствие каждому пути $\gamma \in \bigcup_{v \in V(K,-k)} \Gamma^{+}(v, 2 k+n)$ произвольный двусторонне-бесконечньй 
путь $x(\gamma)=\left(x_{i}, i \in \mathbb{Z}\right)$, для которого $\left(x_{-k}, \ldots, x_{k+n}\right)=\gamma$. Множество $K_{n, \varepsilon}$, состояшее из всех таких $x(\gamma)$, является, очевидно, $(n, \varepsilon)$-плотньм в $K$. Так как

мы получаем (см. (1.20), (1.21))

$$
\# K_{n, \varepsilon} \leqslant \sum_{v \in V(K,-k)} \# \Gamma^{+}(v, 2 k+n),
$$

$$
\begin{aligned}
h\left(X(G), d_{1}, S, K, \varepsilon\right) & \leqslant \varlimsup_{n \rightarrow \infty} \frac{1}{n} \ln \sum_{v \in V(K,-k)} \# \Gamma^{+}(v, 2 k+n) \\
& =\varlimsup_{n \rightarrow \infty} \frac{1}{n} \ln \max _{v \in V(K,-k)} \# \Gamma^{+}(v, 2 k+n),
\end{aligned}
$$

откуда следует (1.26).

Это рассуждение не использует свойства локальной конечности и, по существу, содержится в [17]. Небольшая его модификации приведет к доказательству обратного неравенства.

Подберем для каждого $v \in V(G)$ такие $w_{i} \in V(G), i \leqslant-1$, что множество

$$
F(v)=\left\{x \in X(G): x_{i}=w_{i} \text { при } i \leqslant-1, x_{0}=v\right\}
$$

непусто. Так как граф $G$ локально-конечен вперед, $F(v)$ компактно. Пусть $\varepsilon>0$, $n \in \mathbb{Z}^{+}$и $F_{1}(v)$-произвольное множество, которое $(n, \varepsilon)$-плотно в $F(v)$. Легко понять, что если $\varepsilon<\inf _{v^{\prime}, v^{\prime \prime}} \rho\left(v^{\prime}, v^{\prime \prime}\right)$, то для любого пути $\gamma \in \Gamma^{+}(v, n)$ множество $F_{1}(v)$ должно иметь непустое пересечение с цилиндром $\left\{x \in X(G):\left(x_{0}, \ldots, x_{n}\right)=\gamma\right\}$ (см. $(1.1),(1.2))$. Поэтому $\# F_{1}(v) \geqslant \# \Gamma^{+}(v, n)$, т.е. при достаточно малом $\varepsilon>0$

$$
r_{n}\left(X(G), d_{1}, S, F(v), \varepsilon\right) \geqslant \# \Gamma^{+}(v, n), \quad v \in V(G) .
$$

Отсюда с учетом (1.21)-(1.23) получаем

$$
h\left(X(G), d_{1}, S\right) \geqslant \sup _{v \in V(G)} \varlimsup_{n \rightarrow \infty} \frac{1}{n} \ln \# \Gamma^{+}(v, n),
$$

что вместе с (1.26) приводит к (1.25).

Остается рассмотреть графы, которые не являются локально-конечными вперед. Пусть $G$ - такой граф. Ясно, что для него правая часть (1.26) равна бесконечности, и требуется доказать то же самое для левой части.

Для любой вершины $v \in V$ обозначим через $V^{+}(v)$ множество тех $w \in V$, для которых пара $(v, w)$ служит ребром в $G$. По предположению найдется вершина $v \in V$, для которой \# $V^{+}(v)$ - бесконечное множество. Разобьем его на конечные подмножества $V_{k}^{+}(v)=\left\{v_{k, 1}, \ldots, v_{k, n_{k}}\right\}, k=1,2, \ldots$, выбрав $n_{k}$ так, чтобы выполнялось условие $k=o\left(\ln n_{k}\right)$. Возьмем произвольную периодическую последовательность $x \in X(G)$ с периодом $p$, для которой $x_{0}=v$, и при $k \geqslant 1, n=1, \ldots, n_{k}$ зафиксируем произвольную последовательность $x^{k, n}=\left(x_{i}^{k, n}, i \in \mathbb{Z}\right)$ из $X(G)$, для которой $x_{i}^{k, n}=x_{i}$ при $i \leqslant k p$ и $x_{k p+1}^{k, n}=v_{k, n}$. Пусть $F-$ совокупность полученных таким образом последовательностей. Очевидно, при любом $i \in \mathbb{Z}$ множество $V(F, i)$ конечно и, следовательно, $F$ компактно. Из определения метрики $d_{1}$ следует, что при $k \geqslant 1$ и достаточно малом $\varepsilon \leqslant 1$ мощность любого $(k p+1, \varepsilon)$-плотного в $F$ множества не может быть меньше $n_{k}$, т.е. $r_{k p+1}\left(X(G), d_{1}, S, F, \varepsilon\right) \geqslant n_{k}$. Теперь вследствие выбора $n_{k}$ мы получаем $h\left(X(G), d_{1}, \varepsilon\right)=\infty$, откуда видно, что $h\left(X(G), d_{1}\right)=\infty$. Теорема доказана.

В следующей теореме сравниваются $h\left(X(G), d_{1}, S\right)$ и $h\left(X(G), d_{2}, S\right)$. 
1.12. ТЕОРема [17]. а) Для любого связного графа $G$

$$
h\left(X(G), d_{2}, S\right) \leqslant h\left(X(G), d_{1}, S\right) .
$$

б) Для любых $\alpha, \beta \in \mathbb{R}$, удовлетворяющих условию $1<\alpha \leqslant \beta$, найдется такой связный локально-конечный граф $G$, что

$$
h\left(X(G), d_{1}, S\right)=\beta, \quad h\left(X(G), d_{2}, S\right)=\alpha .
$$

1.13. ЗАмЕчАНИЕ. На сегодняшний день остается открытьм вопрос о взаимоотношениях между энтропией $h(X(G), S)$ (см. замечание 1.4) и энтропией $h(X(G), d, S)$ для тех или иных метрик $d$. Есть основания предполагать, что эти энтропии равны, если $d=d_{2}$.

\section{§. Производящие функции. Неотрицательные матрицы}

В дальнейшем изложении основную роль будут играть периодические точки преобразования $S$ и связанные с ними производяшие функшии.

Пусть

$$
\operatorname{Fix}_{n}(G)=\left\{x \in X(G): S^{n} x=x\right\}, \quad n \geqslant 0 .
$$

Для любого множества $Y \subset X(G)$ и любой функции $f: X(G) \rightarrow \mathbb{R}$ положим

$$
\begin{gathered}
Y_{n}=Y \cap S^{-1}(X(G) \backslash Y) \cap \cdots \cap S^{-n+1}(X(G) \backslash Y), \quad n \geqslant 1, \\
Q_{Y}(G, f, n)=\sum_{x \in Y \cap \operatorname{Fix}_{n}(G)} \exp \sum_{k=0}^{n-1} f\left(S^{k} x\right), \quad n \geqslant 1, \\
Q_{Y}^{0}(G, f, n)=\sum_{x \in Y_{n} \cap \operatorname{Fix}_{n}(G)} \exp \sum_{k=0}^{n-1} f\left(S^{k} x\right), \quad n \geqslant 1
\end{gathered}
$$

(левые части этих равенств будем считать равными нулю, если соответственно $Y \cap \operatorname{Fix}_{n}(G)=\varnothing$ и $\left.Y_{n} \cap \operatorname{Fix}_{n}(G)=\varnothing\right)$. Положив

$$
C(v)=\left\{x \in X(G): x_{0}=v\right\}, \quad v \in V(G)
$$

назовем пару $(G, f)$ допустимой, если $Q_{C(v)}(G, f, n)<\infty$ при всех $v$ и $n$. Ясно, что для допустимой пары $(G, f)$ все $Q_{C(v)}^{0}(G, f, n)$ также конечны.

Введем производящие функции

$$
\begin{aligned}
\varphi(G, f, Y, z) & =\sum_{n=1}^{\infty} Q_{Y}(G, f, n) z^{n} \\
\varphi^{0}(G, f, Y, z) & =\sum_{n=1}^{\infty} Q_{Y}^{0}(G, f, n) z^{n}
\end{aligned}
$$


и при $Y=C(v)$ обозначим радиусы сходимости рядов $(2.3)$ и $(2.4)$ через $R(G, f, v)$ и $R^{0}(G, f, v)$ соответственно. При $Y=X(G)$ будем опускать во всех обозначениях переменную $Y$.

Большая часть содержания этой статьи относится к случаю, когда $f \in \mathscr{F}_{0}^{1}(G)$ (см. определение 1.10). С помошью преобразований редукции и сдвига бо́льшую часть излагаемой здесь теории можно обобщить на любые локальные функции. Обобщение на нелокальные функции представляется нам актуальной и вполне разрешимой задачей.

Каждой функции $f \in \mathscr{F}_{0}^{1}(G)$ можно поставить в соответствие функцию $A_{f}: V(G) \times$ $V(G) \rightarrow \mathbb{R}^{+}$, определяемую следующим образом: если $\left(v, v^{\prime}\right) \notin E(G)$, то $A_{f}\left(v, v^{\prime}\right)=0$; если $\left(v, v^{\prime}\right) \in E(G)$, то $A_{f}\left(v, v^{\prime}\right)=\exp f(x)$, где $x$ - любая последовательность из $X(G)$, для которой $x_{0}=v, x_{1}=v^{\prime}$. Отождествив вершины графа $G$ с натуральными числами, можно считать функцию $A_{f}$ бесконечной неотрицательной матрицей (от способа отождествления ничего зависеть не будет).

Обратно, если задана неотрицательная матрица $A=\left(a_{i j}\right)$ (конечная или бесконечная), можно построить ориентированный граф $G_{A}$, вершины которого - это номера строк (и столбцов) матрищы $A$, а ребра - те пары $(i, j)$, для которых $a_{i j}>0$. Пусть $x=\left(x_{i}, i \in \mathbb{Z}\right) \in X\left(G_{A}\right)$; положив $f_{A}(x)=\ln a_{x_{0} x_{1}}$, мы получим функцию $f \in \mathscr{F}_{0}^{1}\left(G_{A}\right)$. Очевидно, описанное соответствие между неотрицательньми матрицами и парами $(G, f)$, где $G$ - ориентированный граф̆ и $f \in \mathscr{F}_{0}^{1}(G)$, является взаимнооднозначным (с точностью до нумерации вершин). С учетом этого мы часто будем в дальнейшем, имея дело с функциями из $\mathscr{F}_{0}^{1}(G)$, заменять во всех обозначениях пару $(G, f)$ матрицей $A_{f}$.

Матричный язык окажется особенно удобным в этом и следуюших двух параграфах, так как рассматриваемые в них вопросы тесно связаны с теоремой Перрона-Фробениуса для неотрицательных матрищ. Если речь идет о конечной неотрицательной матрице $A$, то согласно этой теореме (см. [18], [19]), $A$ обладает собственньм числом $\lambda(A) \geqslant 0$, которое не меньше абсолютной величины любого другого собственного числа этой матрицы и которому отвечает неотрицательньй собственньй вектор $x_{A}$. Если матрица $A$ неразложима, то $\lambda(A)>0$, вектор $x_{A}$ положителен и собственное подпространство, отвечающее $\lambda(A)$, одномерно.

При попытке обобщения теоремы Перрона-Фробениуса на бесконечные матрищы возникает трудность, состоящая в том, что такая матрища не обязательно определяет ограниченный линейный оператор в каком-либо естественном нормированном пространстве бесконечных числовых последовательностей. Тем не менее Вир-Джоунс $[20],[21]$, применив метод производяших функций и воспользовавшись вместо оператора, отвечающего $A$, аналогом матричных элементов его резольвенты, получил в этом направлении ряд существенных результатов. Сейчас мы изложим некоторые из них в удобной для нас форме, снабдив их необходимыми дополнениями.

Пусть $A=\left(a_{i j}\right)$ - конечная или бесконечная неотрицательная матрица. Назовем ее допустимой, если пара $\left(G_{A}, f_{A}\right)$ допустима. Это, очевидно, означает, что при каждом $n>0$ элементы матрицы $A^{n}$ конечны. В дальнейшем, если не оговорено противное, будем всегда считать рассматриваемые неотрищательные матрищы допустимыми.

При $n \geqslant 1, i, j \in V\left(G_{A}\right)$ рассмотрим множество $\Gamma_{i j}\left(G_{A}, n\right)$, состоящее из $G_{A}$-путей $\gamma=\left(i, i_{1}, \ldots, i_{n-1}, j\right)$ длины $n$, которые ведут из $i$ в $j$, и множество $\Gamma_{i j}^{0}\left(G_{A}, n\right)$, состоящее из тех $\gamma \in \Gamma_{i j}\left(G_{A}, n\right)$, которые не проходят через $j$, т.е. удовлетворяют условию 
$i_{s} \neq j$ при $s \neq 0, n$. Назовем $A$-весом пути $\gamma=\left(i_{0}, i_{1}, \ldots, i_{n}\right)$ число

$$
Q(A, \gamma)=\prod_{s=0}^{n-1} a_{i_{s} i_{s+1}}
$$

и введем производяшие функции

$$
\begin{aligned}
& \varphi_{i j}(A, z)=\sum_{n=1}^{\infty} z^{n} \sum_{\gamma \in \Gamma_{i j}\left(G_{A}, n\right)} Q(A, \gamma)=\sum_{n=1}^{\infty} Q_{i j}(A, n) z^{n}, \\
& \varphi_{i j}^{0}(A, z)=\sum_{n=1}^{\infty} z^{n} \sum_{\gamma \in \Gamma_{i j}^{0}\left(G_{A}, n\right)} Q(A, \gamma)=\sum_{n=1}^{\infty} Q_{i j}^{0}(A, n) z^{n} .
\end{aligned}
$$

Сравнив (2.5), (2.6) с (2.3), (2.4), нетрудно убедиться, что при всяком $n \geqslant 1$ величины $Q_{i i}\left(A_{f}, n\right)$ и $Q_{i i}^{0}\left(A_{f}, n\right)$, определяемые соотношениями $(2.5)$ и $(2.6)$, равны $Q_{C(i)}(G, f, n)$ и $Q_{C(i)}^{0}(G, f, n)$ соответственно, вследствие чего

$$
\varphi_{i i}\left(A_{f}, z\right)=\varphi_{C(i)}(G, f, z), \quad \varphi_{i i}^{0}\left(A_{f}, z\right)=\varphi_{C(i)}^{0}(G, f, z) .
$$

Очевидно, что если матрища $A$ допустима, то все коэффициенты рядов $(2.5),(2.6)$ конечны. Обозначим через $R_{i j}(A)$ и $R_{i j}^{0}(A)$ их радиусы сходимости, которые, как легко понять, удовлетворяют неравенству $R_{i j}(A) \leqslant R_{i j}^{0}(A)$. Заметим также, что при $z$, лежащем в соответствуюшем круге сходимости, справедливы тождества

$$
\varphi_{i j}(A, z)=\sum_{\gamma \in \Gamma_{i j}\left(G_{A}\right)} Q(A, \gamma) z^{l(\gamma)}, \quad \varphi_{i j}^{0}(A, z)=\sum_{\gamma \in \Gamma_{i j}^{0}\left(G_{A}\right)} Q(A, \gamma) z^{l(\gamma)},
$$

где $\Gamma_{i j}\left(G_{A}\right)=\bigcup_{n} \Gamma_{i j}\left(G_{A}, n\right), \Gamma_{i j}^{0}\left(G_{A}\right)=\bigcup_{n} \Gamma_{i j}^{0}\left(G_{A}, n\right)$ и $l(\gamma)$ - длина пути $\gamma$.

Хотя следуюшее утверждение хорошо известно, мы в виде исключения приведем его доказательство (впрочем, очень короткое), так как последнее содержит неравенства, используемые ниже.

2.1. ПРЕДЛОЖЕНИЕ (ср. [20]). Если матрица А неразложима, то

(a) $R_{i j}(A)$ не зависит от $i, j$;

(б) $R_{i j}^{0}(A) \geqslant R_{j j}^{0}(A)$ при любъхх $i, j$.

ДоказАтельство. Вследствие неразложимости $A$ для любых $i, j$ сушествуют путь $\gamma_{i j}$ из $i$ в $j$ и путь $\gamma_{j i}$ из $j$ в $i$. Пусть $i \neq j$. Легко видеть, что при $n>l\left(\gamma_{i j}\right)+l\left(\gamma_{j i}\right)$ вьполняются неравенства

$$
\begin{aligned}
& Q_{i j}(A, n) \geqslant Q\left(A, \gamma_{i j}\right) Q_{i i}\left(A, n-l\left(\gamma_{i j}\right)\right), \\
& Q_{i i}(A, n) \geqslant Q\left(A, \gamma_{j i}\right) Q_{i j}\left(A, n-l\left(\gamma_{j i}\right)\right), \\
& Q_{i i}(A, n) \geqslant Q\left(A, \gamma_{i j}\right) Q\left(A, \gamma_{j i}\right) Q_{j j}\left(A, n-l\left(\gamma_{i j}\right)-l\left(\gamma_{j i}\right)\right),
\end{aligned}
$$


из которых непосредственно вытекает утверждение (a). Считая $\gamma_{j i}$ кратчайшим путем из $j$ в $i$, можно получить аналогичное $(2.8 \mathrm{~b})$ неравенство

$$
Q_{j j}^{0}(A, n) \geqslant Q\left(A, \gamma_{j i}\right) Q_{i j}^{0}\left(A, n-l\left(\gamma_{j i}\right)\right)
$$

которое приводит к утверждению (б).

Пункт (а) предложения 2.1 - одна из простейших “теорем солидарности”, позволяющая всюду ниже писать $R(A)$ вместо $R_{i j}(A)$. Такие теоремы будут встречаться и в дальнейшем. Из очевидного равенства $R_{i i}(A)=R\left(G_{A}, f_{A}, i\right)$ следует, что если граф̆ $G$ связен и $f \in \mathscr{F}_{0}^{1}(G)$, то $R\left(G_{A}, f_{A}, i\right)$ не зависит от $i$ и мы можем опустить $i$ в последнем обозначении, что впредь и будем делать.

2.2. ЗАмЕчАниЕ. По доказанному $R(A)$ в случае неразложимой матрицы $A$ есть радиус сходимости ряда (2.5) при любых $i, j$ и, следовательно,

$$
-\ln R(A)=\varlimsup_{n \rightarrow \infty} n^{-1} \ln Q_{i j}(A, n) .
$$

При некоторых дополнительных условиях это утверждение можно уточнить. Предположим, что граф $G_{A}$ апериодичен (это значит, что наибольший обший делитель $d\left(G_{A}\right)$ длин всех циклов в $G_{A}$ равен единице). Тогда в последнем равенстве при $i=j$ верхний предел можно заменить пределом. Действительно, из определения $Q_{i i}(A, n)$ нетрудно вьвести, что $Q_{i i}(A, n+m) \geqslant Q_{i i}(A, n) Q_{i i}(A, m)$ при любых $m$ и $n$, а из апериодичности следует, что $Q_{i i}(A, n)>0$ при всех достаточно больших $n$ (скажем, при $\left.n \geqslant n_{0}\right)$. Поэтому последовательность чисел $b_{n}=-\ln Q_{i i}(A, n), n \geqslant n_{0}$, обладает свойством субаддитивности: $b_{m+n} \leqslant b_{m}+b_{n}$, а тогда сушествует предел $\lim _{n \rightarrow \infty} b_{n} / n$ (см., например, $\left[2\right.$, с. 122]). Если $d\left(G_{A}\right)=d>1$, то все сказанное сохранится после замены $n$ на $n d$.

2.3. ЗАмЕчАниЕ. Если $A$ - конечная неразложимая неотрицательная матрища с максимальным собственным числом $\lambda(A)$, то $R(A)=[\lambda(A)]^{-1}$. В самом деле, так как матрища $A$ конечна, из предложения 2.1 следует, что $R(A)$ есть радиус сходимости ряда $\sum_{n=1}^{\infty} \operatorname{tr}\left(A^{n}\right) z^{n}$. Приведя $A$ к жордановой форме $A_{1}$, убеждаемся затем, что $\operatorname{tr}\left(A_{1}^{n}\right)=\operatorname{tr}\left(A^{n}\right)$. Но в силу теоремы Перрона-Фробениуса (см. [18, гл. 13]) $\varlimsup_{n \rightarrow \infty}\left|\operatorname{tr}\left(A_{1}^{n}\right)\right|^{1 / n}=\lambda(A)$.

2.4. ОПРеДЕЛЕниЕ (ср. [20]). Неразложимая неотрицательная матрища $A$ называется возвратной, если найдется такое $i$, что $\varphi_{i i}(A, R(A))=\infty$, и положительно-возвратной, если найдется такое $i$, что $Q_{i i}(A, n)[R(A)]^{n} \nrightarrow 0$ при $n \rightarrow \infty$. $\mathrm{B}$ противном случае $A$ назьвается соответственно невозвратной и нуль-возвратной.

Из определения следует, что если матрища $A$ возвратна, то $R(A)>0$. Очевидно, это определение сформулировано по аналогии с частным случаем, когда $A$ - стохастическая матрица. Как показывает следующая ниже теорема солидарности, вместо слов "найдется такое $i$ " в нем можно писать "для любого $i$ ". 
2.5. ПРЕДЛОЖЕНИЕ [20]. Если матрица А неразложима, то каждое из соотношений

$$
\varphi_{i j}(A, R(A))=\infty, \quad \lim _{n \rightarrow \infty} Q_{i j}(A, n)[R(A)]^{n}=0
$$

выполняется или не выполняется одновременно для всех пар $(i, j)$.

Теперь выразим возвратность и положительную возвратность в терминах функций $\varphi_{i i}^{0}$.

2.6. ЛЕмма. Пусть $A$ - неразложимая неотрицательная матрица и $i \in \mathbb{N}$. Тогда

1) равенства $\varphi_{i i}^{0}(A, R(A))=1$ и $\varphi_{i i}(A, R(A))=\infty$ әквивалентнь;

2) при всех $і$ выполняется равенство $R(A)=\min \left\{R_{i i}^{0}(A), t_{i i}(A)\right\}$, где $t_{i i}(A)$ определяется соотношением $\varphi_{i i}^{0}\left(A, t_{i i}(A)\right)=1$, если $\varphi_{i i}^{0}\left(A, R_{i i}^{0}(A)\right) \geqslant 1, u$ $t_{i i}(A)=\infty$ в противном случае.

ДОКАЗАТЕЛЬСТвО. Очевидно, при любых $i, j$

$$
Q_{j i}(A, n)= \begin{cases}Q_{j i}^{0}(A, n)+\sum_{k=1}^{n-1} Q_{j i}^{0}(A, k) Q_{i i}(A, n-k), & n \geqslant 2, \\ a_{j i}, & n=1,\end{cases}
$$

откуда получаем

$$
\varphi_{i i}(A, z)=\varphi_{i i}^{0}(A, z)\left[1-\varphi_{i i}^{0}(A, z)\right]^{-1}, \quad|z|<R(A), \quad i=1,2, \ldots .
$$

Пользуясь неотрицательностью коэффициентов $Q_{i i}(A, \cdot)$ и $Q_{i i}^{0}(A, \cdot)$, из $(2.10)$ (при $i=j$ ) легко вьвести утверждение 1$)$. Что касается утверждения 2 ), то оно следует из равенства

$Q_{i i}(A, n)=Q_{i i}^{0}(A, n)+\sum_{k=2}^{n} \sum_{n_{1}+\cdots+n_{k}=n} Q_{i i}^{0}\left(A, n_{1}\right) \cdots Q_{i i}^{0}\left(A, n_{k}\right), \quad i \in V\left(G_{A}\right), n \geqslant 2$, которое, в свою очередь, вьводится по индукции из (2.9).

2.7. ТеОРема [20]. Положительная возвратность неразложимой матрицы А равносильна кажсдому из следующих трех свойств:

1) $\varphi_{i i}^{0}(A, R(A))=1,\left.\frac{d}{d t} \varphi_{i i}^{0}(A, t)\right|_{t=R(A)}<\infty$

2) либо $\varphi_{i i}^{0}\left(A, R_{i i}^{0}(A)\right)>1$, либо $\varphi_{i i}^{0}\left(A, R_{i i}^{0}(A)\right)=1,\left.\frac{d}{d t} \varphi_{i i}^{0}(A, t)\right|_{t=R_{i i}^{0}(A)}<\infty$;

3) существуют векторьи $y^{\prime}(A)=\left(y_{n}^{\prime}(A), n=1,2, \ldots\right)$ и $y^{\prime \prime}(A) \stackrel{i \imath}{=}\left(y_{n}^{\prime \prime}(A)\right.$, $n=1,2, \ldots)$ с положительными компонентами, удовлетворяющие соотношениям

$$
\begin{gathered}
\sum_{n=1}^{\infty} a_{j n} y_{n}^{\prime}(A)=[R(A)]^{-1} y_{j}^{\prime}(A), \sum_{n=1}^{\infty} a_{n j} y_{n}^{\prime \prime}(A)=[R(A)]^{-1} y_{j}^{\prime \prime}(A), \quad j=1,2, \ldots, \\
\sum_{n=1}^{\infty} y_{n}^{\prime}(A) y_{n}^{\prime \prime}(A)=1
\end{gathered}
$$


и определяемье однозначно с точностью до множителя. При әтом каждое из свойств 1) и 2) имеет место для всех $i$, если это верно хотя бь для одного $i$.

Таким образом, для положительно-возвратных матриц справедлив аналог теоремы Перрона-Фробениуса, в котором $1 / R(A)$ играет роль максимального собственного числа.

Из теоремы 2.7 вытекает, что если $A=A_{f}, f \in \mathscr{F}_{0}^{1}(G)$, и $R(A)>0$, то функция $f$ гомологична некоторой ограниченной сверху функции $f_{1} \in \mathscr{F}_{0}^{1}(G)$, т.е. $f(x)=$ $f_{1}(x)+g(S x)-g(x), x \in X(G)$, где $g$ - борелевская функщия на $X(G)$. В самом деле, если матрица $A$ положительно-возвратна, то матрища $A^{\prime}$ с элементами $a_{i j}^{\prime}=$ $R(A) a_{i j} y_{j}^{\prime}(A) / y_{i}^{\prime}(A)$ является стохастической, и можно положить

$$
f_{1}(x)=\ln a_{x_{0} x_{1}}^{\prime}-\ln R(A), \quad g(x)=-\ln y_{x_{0}}^{\prime}(A), \quad x=\left(x_{i}, i \in \mathbb{Z}\right) \in X(G) .
$$

Остается заметить, что если $R\left(A_{f}\right)>0$, то матрица $A_{f+t 1_{C(v)}}$, где $v$ - произвольная вершина графа $G$ и $C(v)=\left\{x \in X(G): x_{0}=v\right\}$, положительно-возвратна при достаточно большом $t>0$.

Введем теперь еще одно важное понятие.

2.8. ОПРЕДЕЛЕНИЕ. Назовем положительно-возвратную матрицу А устойчивоположительной, если найдется такое $i$, что

$$
\varphi_{i i}^{0}\left(A, R_{i i}^{0}(A)\right)>1
$$

В противном случае назовем ее неустойчиво-положительной.

Из п. 2) леммы 2.6 вытекает, что (2.11) можно переписать в виде

$$
R_{i i}^{0}(A)>R(A)
$$

Несмотря на то, что $R_{i i}^{0}(A)$ может зависеть от $i$, здесь также верна теорема солидарности.

2.9. ПРЕДЛОЖЕНИЕ (ср. [20]). Для неразложимой матриць А условие устойчивой положительности (2.11) выполняется или не выполняется одновременно при всеx $i$.

2.10. СлЕДСТвИЕ. Для неразложимой матрицы А условие неустойчивой положительности

$$
\varphi_{i i}^{0}\left(A, R_{i i}^{0}(A)\right)=1,\left.\quad \frac{d}{d t} \varphi_{i i}^{0}(A, t)\right|_{t=R_{i i}^{0}(A)}<\infty
$$

выполняется или не выполняется одновременно при всех $i$. 
2.11. ЗАмечАниЕ. Пусть $A$ - неразложимая матрица и $a_{i j}-$ любой из ее положительных элементов. Заменив его произвольным $a_{i j}^{\prime}>0$ и оставив неизменньми остальные элементы матрицы $A$, получим новую матрицу $A^{\prime}$ с $G_{A^{\prime}}=G_{A}$, для которой, как легко понять, $R_{i i}^{0}\left(A^{\prime}\right)=R_{i i}^{0}(A)$. Очевидно, при $z \in\left(0, R_{i i}^{0}(A)\right)$ имеем $\varphi_{i i}^{0}\left(A^{\prime}, z\right)<\varphi_{i i}^{0}(A, z)$, если $a_{i j}^{\prime}<a_{i j}$, и $\varphi_{i i}^{0}\left(A^{\prime}, z\right)>\varphi_{i i}^{0}(A, z)$, если $a_{i j}^{\prime}>a_{i j}$. Ясно, кроме того, что $\varphi_{i i}^{0}\left(A^{\prime}, z\right)$ непрерывно зависит от $a_{i j}^{\prime}$ при фиксированном $z$ из круга сходимости. Поэтому свойства невозвратности и устойчивой положительности матрицы $A$ (при которых соответственно $\varphi_{i i}^{0}\left(A, R_{i i}^{0}(A)\right)<1$ и $\varphi_{i i}^{0}\left(A, R_{i i}^{0}(A)\right)>1$ ) будут наследоваться матрицей $A^{\prime}$, если $a_{i j}^{\prime}$ и $a_{i j}$ достаточно близки, и, наоборот, соотношение $\varphi_{i i}^{0}\left(A, R_{i i}^{0}(A)\right)=1$ не выдерживает замены $A$ на $A^{\prime}$, если только $a_{i j}^{\prime} \neq a_{i j}$. Отсюда, в частности, следует, что малое возмущение конечного числа элементов невозвратной или устойчиво-положительной матрицы $A$ снова приводит соответственно к невозвратной и устойчиво-положительной матрице. Более того, последнее свойство сохраняется и при замене каждого элемента $a_{i j}$ на $a_{i j}^{\prime}$, где $1-\varepsilon<a_{i j}^{\prime} / a_{i j}<1+\varepsilon$ и $\varepsilon$ достаточно мало. Этим оправдывается введенньй нами термин "устойчивая положительность”. Впрочем, это свойство можно было бы с равным успехом назвать и "устойчивой возвратностью" (что и сделано в [22]).

Как мы увидим в дальнейшем, устойчиво-положительные матрицы во многом близки по своим свойствам к конечным матрицам, что часто позволяет получать для них результаты, которые, вообще говоря, неверны для более широких классов неотрицательных матриц. По этой причине следуюший параграф̆ будет целиком посвящен устойчиво-положительным матрицам. А данньй параграф мы завершим рассмотрением одного важного технического вопроса, связанного с отображением $n$-редукции.

Пусть, как и раньше, $G$ - связный ориентированньй граф с множеством вершин $V(G)$ и множеством ребер $E(G)$ (мы всегда будем предполагать, что $\# V(G)>1$ ). В $\S 1$ было введено взаимнооднозначное отображение $\Phi_{n}, n \geqslant 1$, переводящее марковское множество $X(G)$ в марковское множество $\Phi_{n} X(G)=X\left(G_{n}\right)$, где граф $G_{n}$ однозначно определяется последним равенством (что позволяет положить по определению $\left.G_{n}=\Phi_{n} G\right)$. Ясно, что вершинами этого графа служат все $G$-пути длины $n-1$, а ребрами - все пары путей $\gamma=\left(i_{0}, \ldots, i_{n-1}\right), \gamma^{\prime}=\left(i_{0}^{\prime}, \ldots, i_{n-1}^{\prime}\right)$, для которых $i_{0}^{\prime}=i_{1}, i_{1}^{\prime}=i_{2}, \ldots, i_{n-2}^{\prime}=i_{n-1}$. Отображение $\Phi_{n}$ естественным образом переносится и на функции. Легко проверить, что это отображение, где бы оно ни рассматривалось - на марковских множествах, графах или функциях, - удовлетворяет тождеству $\Phi_{k} \Phi_{l}=\Phi_{k+l-1}$. Мы покажем, что свойства матрицы $A_{f}$, где $f \in \mathscr{F}_{0}^{1}(G)$, наследуются матрицей $A_{\Phi_{k}} f$ при любом $k \geqslant 1$.

2.12. ПреДЛОЖЕнИЕ. Если $G-$ связныц ираф $f \in \mathscr{F}_{0}^{1}(G) u k \geqslant 2$, mo

$$
R\left(A_{\Phi_{k} f}\right)=R\left(A_{f}\right)
$$

и для того чтобь матрича $A_{\Phi_{k}}$ ббила невозвратной, нуль-возвратной, неустойчиво-положительной и устойчиво-положительной, необходимо и достаточно, чтобы соответствующим свойством обладала матрица $A_{f}$.

ДокАЗАТЕЛЬСТВО. Ввиду соотношения $\Phi_{k} \Phi_{l}=\Phi_{k+l-1}$ можно ограничиться случаем $k=2$. Положим $G_{2}=\Phi_{2} G, f_{2}=\Phi_{2} f, A=A_{f}, A_{2}=A_{f_{2}}$. Пусть $(i, j)$ - произвольное ребро графа $G$ с $j \neq i$. Пара $(i, j)$ образует вершину графа $G_{2}$ и любой цикл 
$\gamma \in \Gamma_{(i, j)(i, j)}\left(G_{2}, n\right), n \geqslant 2$, имеет вид

$$
\gamma=\left((i, j),\left(i_{1}, i_{2}\right), \ldots,\left(i_{n-1}, i_{n}\right),(i, j)\right)
$$

где $\gamma^{\prime}=\left(i_{1}, \ldots, i_{n}\right)$ - путь, принадлежаший $\Gamma_{j i}(G, n-1)$. Очевидно, переход от $\gamma$ к $\gamma^{\prime}$ порождает взаимнооднозначное соответствие между множествами $\Gamma_{(i, j)(i, j)}\left(G_{2}, n\right)$ и $\Gamma_{j i}(G, n-1)$. Поэтому (см. определение $\left.A_{2}\right)$

$$
Q_{(i, j)(i, j)}\left(A_{2}, n\right)=a_{i j} Q_{j i}(A, n-1), \quad n \geqslant 2, \quad Q_{(i, j)(i, j)}\left(A_{2}, 1\right)=0,
$$

вследствие чего

$$
R\left(A_{2}\right)=R(A), \quad \varphi_{(i, j)(i, j)}\left(A_{2}, z\right)=a_{i j} z \varphi_{j i}(A, z) .
$$

Нам потребуется еще соотношение между суммами весов некоторых простых циклов, но в отличие от (2.14) оно будет иметь вид неравенства. Возьмем те же $i, j$, что и раньше, и зафиксируем кратчайший путь $\gamma_{j i}=\left(j_{0}, \ldots, j_{l}\right)$, ведуший из $j$ в $i$. Пусть на этот раз

$$
\gamma=\left(i, i_{1}, \ldots, i_{n-1}, i\right) \in \Gamma_{i i}^{0}(G, n), n \geqslant 2, \quad \gamma^{\prime}=\left(\left(i, i_{1}\right),\left(i_{1}, i_{2}\right), \ldots,\left(i_{n-1}, i\right),(i, j)\right)
$$

Положим

$$
\gamma^{\prime \prime}= \begin{cases}\gamma^{\prime}, & \text { если } i_{1}=j, \\ \left(\left(i, j_{0}\right),\left(j_{0}, j_{1}\right), \ldots,\left(j_{l-1}, i\right)\right) \gamma^{\prime}, & \text { если } i_{1} \neq j .\end{cases}
$$

Очевидно, в первом случае $\gamma^{\prime \prime} \in \Gamma_{(i, j)(i, j)}^{0}\left(G_{2}, n\right), Q\left(A_{2}, \gamma^{\prime \prime}\right)=Q(A, \gamma)$, во втором случае $\gamma^{\prime \prime} \in \Gamma_{(i, j)(i, j)}^{0}\left(G_{2}, n+l+1\right), Q\left(A_{2}, \gamma^{\prime \prime}\right)=a_{i j} Q(A, \gamma) Q\left(A, \gamma_{j i}\right)$ и в обоих случаях разньм $\gamma$ отвечают разные $\gamma^{\prime \prime}$. Отсюда получаем неравенство

$$
Q_{i i}^{0}(A, n) \leqslant Q_{(i, j)(i, j)}^{0}\left(A_{2}, n\right)+\left[a_{i j} Q\left(A, \gamma_{j i}\right)\right]^{-1} Q_{(i, j)(i, j)}^{0}\left(A_{2}, n+l+1\right), \quad n \geqslant 2,
$$

приводящее к вьводу, что

$$
R_{(i, j)(i, j)}^{0}\left(A_{2}\right) \leqslant R_{i i}^{0}(A)
$$

Из (2.14) сразу следует, что как возвратность, так и положительная возвратность матришы $A_{2}$ равносильны соответствуюшим свойствам матрицы $A$ (см. определение 2.4 и предложение 2.5).

Чтобы доказать то же самое для устойчивой и неустойчивой положительности, получим из (2.15) с помощью (2.10) соотношение

$$
\varphi_{(i, j)(i, j)}^{0}\left(A_{2}, z\right)=\frac{a_{i j} z \varphi_{j i}^{0}(A, z)}{1-\varphi_{i i}^{0}(A, z)+a_{i j} z \varphi_{j i}^{0}(A, z)} .
$$

Предположим теперь, что матрица $A$ устойчиво-положительна, т.е.

$$
R_{j i}^{0}(A) \geqslant R_{i i}^{0}(A)>R(A)
$$


(см. определение 2.8 и п. (б) предложения 2.1). Тогда найдется такое $r>R(A)$, что в круге $|z|<r$ числитель и знаменатель дроби, стоящей в правой части (2.17), аналитичны и, следовательно, функщия $\varphi_{(i, j)(i, j)}^{0}\left(A_{2}, \cdot\right)$ мероморфна. В силу п. 2) предложения 3.5 (при доказательстве предложения 3.5 используются лишш утверждения, предшествуюшие предложению 2.12) это означает, что матрица $A_{2}$ устойчиво-положительна.

Если, наконец, матрица $A$ неустойчиво-положительна, то $R_{i i}^{0}(A)=R(A)($ см. следствие 2.10$)$, откуда с учетом (2.15), (2.16) получаем

$$
R(A)=R\left(A_{2}\right) \leqslant R_{(i, j)(i, j)}^{0}\left(A_{2}\right) \leqslant R_{i i}^{0}(A)=R(A) .
$$

Следовательно, $R\left(A_{2}\right)=R_{(i, j)(i, j)}^{0}\left(A_{2}\right)$, а так как по доказанному положительная возвратность матрицы $A$ влечет за собой положительную возвратность матрицы $A_{2}$, последняя может быть лишь неустойчиво-положительной. Предложение доказано.

Введем еще несколько обозначений. Пусть $G$ - произвольньй ориентированньй граф̆ и $\gamma=\left(v_{0}, \ldots, v_{l}\right), l \geqslant 2,-$ произвольньй $G$-путь. При $0 \leqslant s \leqslant t \leqslant l$ положим $\gamma_{s}^{t}=\left(v_{s}, \ldots, v_{t}\right)$. Очевидно, $\gamma_{s}^{t}$ также будет $G$-путем. Если $\gamma-$ цикл, то ему можно поставить в соответствие бесконечную периодическую последовательность $\operatorname{per}(\gamma)=$ $\left(x_{i}, i \in \mathbb{Z}\right) \in X(G)$, определяемую равенством $\left(x_{r l}, x_{r l+1}, \ldots, x_{(r+1) l}\right)=\gamma, r \in \mathbb{Z}$. Если $x=\left(x_{i}, i \in \mathbb{Z}\right)$ - произвольная точка из $X(G)$, то при каждом $n \geqslant 0$ ей отвечает конечная последовательность $\gamma^{x, n}=\left(x_{0}, \ldots, x_{n}\right)$, которая, очевидно, является $G$-путем. Наконец, для любых $v \in V(G), \gamma \in \Gamma(G)$ положим

$$
C(v)=\left\{x \in X(G): x_{0}=v\right\}, \quad C(\gamma)=\left\{x \in X(G):\left(x_{0}, \ldots, x_{l(\gamma)}\right)=\gamma\right\} .
$$

2.13. ПРЕДЛОЖЕНИЕ. Пусть заданы связный ориентированный граф $G$ и функчия $f \in \mathscr{F}_{0}^{m}(X(G)), m \geqslant 1$, удовлетворяющие любому из следующих условии:

(a) $m=1$;

(б) $m \geqslant 1, G$ локально-конечен;

(в) $m \geqslant 1, f$ ограничена.

Тогда для любих $k \geqslant m, v \in V(G)$ и любого пути $\gamma_{0}$ длинь $k$, вьходящего из $v$, найдутся такие числа $B, b$ и $n_{0}$, что

$$
Q_{C(v)}(G, f, n) \leqslant B Q_{C\left(\gamma_{0}\right)}(G, f, n+b) \leqslant B Q_{C(v)}(G, f, n+b), \quad n \geqslant n_{0} .
$$

ДокаЗАТЕльСТво. Поскольку $C\left(\gamma_{0}\right) \subset C(v)$, правое неравенство в $(2.19)$ вьполняется при любых $B, b$, и в доказательстве нуждается лишь левое. Пусть $v^{+}-$вершина, в которую входит путь $\gamma_{0}$ (см. определение 1.1). Возьмем, пользуясь связностью $G$, какой-нибудь путь $\gamma^{+}$, ведущий из $v^{+}$в $v$, и образуем путь $\gamma_{1}=\gamma_{0} \gamma^{+}$, длина которого равна $l_{1}=k+l^{+}$, где $l^{+}-$длина пути $\gamma^{+}$. Ясно, что $\gamma_{1} \in \Gamma_{v v}\left(G, k+l^{+}\right)$.

Всякой точке $x \in C(v) \cap \operatorname{Fix}_{n}(G), n \geqslant 1$, поставим в соответствие точку $x^{\prime}=$ $\operatorname{per}\left(\gamma_{1} \gamma^{x, n}\right) \in X(G)$. Очевидно, $x^{\prime} \in C\left(\gamma_{0}\right) \cap \operatorname{Fix}_{n+l_{1}}(G)$ и разным $x$ отвечают разные $x^{\prime}$. Положив

$$
u_{n}(x)=\exp \sum_{j=0}^{n-1} f\left(S^{j} x\right), \quad u_{n+l_{1}}\left(x^{\prime}\right)=\exp \sum_{j=0}^{n+l_{1}-1} f\left(S^{j} x^{\prime}\right),
$$


сравним $u_{n}(x)$ и $u_{n+l_{1}}\left(x^{\prime}\right)$.

Поскольку $f \in \mathscr{F}_{0}^{m}(G)$, сушествует функщия $\tilde{f}$, определенная на $G$-путях длины $m$, для которой $f(x)=\tilde{f}\left(\gamma^{x, m}\right)$. В этих обозначениях при любом $n>m$

$$
\begin{aligned}
& u_{n}(x)=\exp [\left.\sum_{i=0}^{n-m} \tilde{f}\left(\left(\gamma^{x, n}\right)_{i}^{i+m}\right)+\sum_{i=n-m+1}^{n-1} \tilde{f}\left(\left(\gamma^{x, n}\right)_{i}^{n}\left(\gamma^{x, n}\right)_{0}^{m-n+i}\right)\right], \\
& u_{n+l_{1}}\left(x^{\prime}\right)=\exp \left[\sum_{i=0}^{l_{1}-m} \tilde{f}\left(\left(\gamma_{1}\right)_{i}^{i+m}\right)+\sum_{i=l_{1}-m+1}^{l_{1}-1} \tilde{f}\left(\left(\gamma_{1}\right)_{i}^{l_{1}}\left(\gamma^{x, n}\right)_{0}^{m-l_{1}+i}\right)\right. \\
&\left.+\sum_{i=0}^{n-m} \tilde{f}\left(\left(\gamma^{x, n}\right)_{i}^{i+m}\right)+\sum_{i=n-m+1}^{n-1} \tilde{f}\left(\left(\gamma^{x, n}\right)_{i}^{k}\left(\gamma_{1}\right)_{0}^{m-n+i}\right)\right]
\end{aligned}
$$

(суммы, в которых верхний индекс больше нижнего, считаются равными нулю). Для двух сумм в правой части (2.20) и четырех сумм в правой части (2.21) введем обозначения $S_{1,1}, S_{1,2}$ и $S_{2,1}, S_{2,2}, S_{2,3}, S_{2,4}$ соответственно. Заметим, что $S_{21}$ не зависит от $x$ и $n$. Поэтому при $m=1$ (см. условие (a)), когда $S_{1,2}=S_{2,2}=S_{2,4}=0$, мы имеем $u_{n}(x)=B_{1} u_{n+l_{1}}\left(x^{\prime}\right)$, где $B_{1}=\exp \left(-S_{21}\right)$. В случае (б) сушествует лишш конечное число путей длины $<m$, входяших в вершину $v$ или выходяших из нее. Поэтому $S_{1,2}, S_{2,2}$ и $S_{2,4}$ как функции от $x$ и $n$ принимают конечное число положительных значений, и, следовательно, $u_{n}(x) \leqslant B_{2} u_{n+l_{1}}\left(x^{\prime}\right)$, где $B_{2}$ не зависит от $x$ и $n$. Наконец, в случае (в) все значения сумм $S_{1,2}, S_{2,2}$ и $S_{2,4}$ лежат между $m \min _{x \in X(G)} f(x)$ и $m \max _{x \in X(G)} f(x)$, и мы приходим к тому же неравенству. Остается заметить, что из него непосредственно вытекает (2.19), если положить $b=l_{1}, n_{0}=m+1$. Предложение доказано.

2.14. СлЕДСТВИЕ. Если выполнены условия предложсения 2.13, то

$$
R\left(\Phi_{k} G, \Phi_{k} f\right)=R(G, f, v), \quad v \in V(G) .
$$

\section{$\S$ 3. Критерии устойчивой положительности}

В этом параграфе мы с разных сторон охарактеризуем устойчиво-положительные матришы (см. также $\S 10)$. Некоторые из приводимых ниже свойств этих матриц, внешне совершенно различные, в действительности оказьваются эквивалентными самой устойчивой положительности и, значит, друг другу. Начнем с простейшего случая.

3.1. ПРЕДЛОЖЕНИЕ. Всякая конечная неразложимая матрица А устойчиво-положительна, и для такой матриць функции $\varphi_{i i}(A, \cdot)$ и $\varphi_{i i}^{0}(A, \cdot)$ рациональньи.

ДокАЗАТЕЛЬСтво. Пусть $A$ - матрица, о которой идет речь в формулировке, и $V=V\left(G_{A}\right)-$ множество вершин отвечаюшего ей графа $G_{A}$. Из очевидного равенства

$$
Q_{i j}(A, n)=\sum_{k \in V} a_{i k} Q_{k j}(A, n-1), \quad i, j \in V, \quad n \geqslant 2,
$$


вытекает, что

$$
\varphi_{i j}(A, z)=z\left[a_{i j}+\sum_{k \in V} a_{i k} \varphi_{k j}(A, z)\right] .
$$

При фиксированном $j$ эти соотношения можно рассматривать как систему линейных уравнений относительно неизвестных $\varphi_{i j}(A, z)$. Так как это конечная система, ее можно решать по правилу Крамера, откуда видно, что функции $\varphi_{i j}(A, \cdot)$ рациональны. В силу (2.10) функции $\varphi_{i i}^{0}(A, \cdot)$ тоже рациональны. Следовательно, $R_{i i}^{0}(A)=\left|z_{i}\right|$, где $z_{i}$ - ближайший к началу координат полюс функции $\varphi_{i i}^{0}(A, \cdot)$, если у нее действительно есть полюса, и $R_{i i}^{0}(A)=\infty$, если эта функция - многочлен. Так как в круге $|z|<\left|z_{i}\right|$ эта функция разлагается в ряд с неотрицательными коэффициентами, можно утверждать, что

$$
\varphi_{i i}^{0}\left(A,\left|z_{i}\right|\right) \geqslant \lim _{z \rightarrow z_{i}}\left|\varphi_{i i}^{0}(A, z)\right|=\infty .
$$

Тем самым доказано, что $\varphi_{i i}^{0}\left(A, R_{i i}^{0}(A)\right)=\infty$ при всех $i \in V$ и, значит, матрица $A$ устойчиво-положительна.

Теперь остановимся на структуре множества $\mathscr{R}(A)=\left\{R_{i i}^{0}(A), i=1,2, \ldots\right\}$. Как следует из леммы 2.6 и теоремы 2.7, это множество во всех случаях, кроме случая устойчивой положительности, состоит из единственной точки $R(A)$. В [21] приведен пример бесконечной матрицы $A$, для которой множество $\mathscr{R}(A)$ бесконечно. Следующее предложение показывает, что для любой устойчиво-положительной матрицы дело обстоит точно так же.

3.2. ПРЕДЛОЖЕНИЕ. Пусть $A$ - неразложимая устойчиво-положсительная матрица. Тогда для всякого $\varepsilon>0$ множество таких $i$, что $R_{i i}^{0}(A)>R(A)+\varepsilon$, конечно.

ДокАЗАТЕЛЬСтво. Зафиксировав произвольное $j, \operatorname{paccмотрим~функцию~} \varphi_{j j}(A, \cdot)$. Так как матрица $A$ устойчиво-положительна, выполняются неравенство $R_{j j}^{0}(A)>$ $R(A)$ (см. предложение 2.5 и п. 2 леммы 2.6) и равенство $\varphi_{j j}^{0}(A, R(A))=1$. Поэтому (см. (2.7)) найдутся конечное множество путей $\Gamma_{\varepsilon} \subset \Gamma_{j j}^{0}\left(G_{A}\right)$ и число $t_{\varepsilon}>0$, для которых

$$
R(A)<t_{\varepsilon}<R(A)+\varepsilon, \quad \varphi_{\varepsilon}\left(t_{\varepsilon}\right)=1
$$

где

$$
\varphi_{\varepsilon}(z)=\sum_{\gamma \in \Gamma_{\varepsilon}} Q(A, \gamma) z^{l(\gamma)}
$$

$\left(t_{\varepsilon}>0\right.$ определяется равенством $\varphi_{\varepsilon}\left(t_{\varepsilon}\right)=1$ однозначно).

Обозначим через $A_{\varepsilon}$ произвольную неразложимую конечную подматрицу матрищы $A$, для которой $\Gamma_{\varepsilon} \subset \Gamma_{j j}^{0}\left(A_{\varepsilon}\right)$. В силу теоремы $2.7 R\left(A_{\varepsilon}\right) \leqslant t_{\varepsilon}$.

Для произвольной вершины $i \notin V\left(G_{A_{\varepsilon}}\right)$ зафиксируем кратчайший путь $\gamma_{i j}$ из $i$ в $j$ и кратчайший путь $\gamma_{j i}$ из $j$ в $i$ (если кратчайших путей несколько, возьмем в каждом случае любой из них). Очевидно, эти пути содержат $i$ лишш в качестве начальной и конечной точки соответственно. Поэтому для любого $z>0$

$$
\varphi_{i i}^{0}(A, z)=\sum_{\gamma \in \Gamma_{i i}^{0}\left(G_{A}\right)} Q(A, \gamma) z^{l(\gamma)} \geqslant Q\left(A, \gamma_{i j}\right) Q\left(A, \gamma_{j i}\right) z^{l\left(\gamma_{i j}\right)+l\left(\gamma_{j i}\right)} \varphi_{j j}\left(A_{\varepsilon}, z\right),
$$


откуда видно, что $R_{i i}^{0}(A) \leqslant R\left(A_{\varepsilon}\right)$. Вместе с $(3.1)$ и полученным вьше неравенством $R\left(A_{\varepsilon}\right) \leqslant t_{\varepsilon}$ это приводит к выводу, что $R_{i i}^{0}(A)<R(A)+\varepsilon$. Предложение доказано.

В силу этого предложения бесконечность множества $\mathscr{R}(A)$ может служить критерием устойчивой положительности. Прежде чем переходить к другим критериям, напомним следующее определение.

3.3. ОПРеДЕЛЕниЕ. Пусть $G$ - ориентированный граф̆ и $d(G)$ - наибольший обший делитель длин всех его циклов. Число $d(G)$ назьвается периодом граффа $G$; если $d(G)=1$, то $G$ называется апериодическим графом.

В случае, когда неотрищательной матрице $A$ отвечает апериодический граф $G_{A}$, будем назьвать апериодической и саму эту матрицу.

Легко видеть, что если граф $G$ связен, то $d(G)$ совпадает с наибольшим общим делителем длин циклов, составляющих каждое из множеств $\Gamma_{v v}(G)$ и $\Gamma_{v v}^{0}(G)$, где $v-$ произвольная вершина.

Следуюший простой факт потребуется не только здесь, но и в гл. 3.

3.4. Лемма. Пусть $\varphi(z)$ и $R_{0} \in(0, \infty)$ - соответственно сумма и радиус сходимости степенного ряда с коэффициентами $a_{n} \geqslant 0, n=1,2, \ldots$, и при некотором $R<R_{0}$ выполняется равенство $\varphi(R)=1$. Тогда найдется такое $r \in\left(R, R_{0}\right)$, что множество нулей функции $1-\varphi(z)$, лежащих в круге $|z| \leqslant r$, имеет вид

$$
U=\{R \exp (2 \pi i k / d), 0 \leqslant k \leqslant d-1\},
$$

где $d$ - наибольиий общий делитель (НОД) тех $n$, для которьх $a_{n}>0$, причем все эти нули - простые.

ДоКАЗАТЕЛЬСТВо. Из определения $U$ и $d$ видно, что $\varphi(z)=\varphi(R)=1$ при всех $z \in U$, авследствие неотрицательности коэффициентов $a_{n}$ и равенства $\varphi(R)=1$ функция $1-\varphi(z)$ не может иметь нулей в круге $|z|<R$. Поэтому найдется такое $r \in$ $\left(R, R_{0}\right)$, что все ее нули в круге $|z|<r$ лежат на окружности $|z|=R$. Положим $J=\left\{n: a_{n}>0\right\}$ и пусть при некотором $z=R \exp (2 \pi i \alpha)$, где $0 \leqslant \alpha<1$, выполняется равенство $\varphi(z)=1$. Так как $a_{n} \geqslant 0$ при всех $n$, это возможно лишш в случае, когда $n \alpha \in \mathbb{Z}$ при всех $n \in J$. Пусть теперь $s \geqslant 1$ и $n_{1}, \ldots, n_{s}$ таковы, что НОД $\left\{n_{1}, \ldots, n_{s}\right\}=d$. Подобрав $k_{1}, \ldots, k_{s} \in \mathbb{Z}$ так, что $k_{1} n_{1}+\cdots+k_{s} n_{s}=d$, и воспользовавшись условием $n_{j} \alpha \in \mathbb{Z}, j=1, \ldots, s$, приходим к вьводу, что $d \alpha \in \mathbb{Z}$, т.е. $\alpha=k / d$, где $k \in\{0, \ldots, d-1\}$.

Что касается простоты нулей функции $1-\varphi(z)$ в точках $z \in U$, то она вытекает из очевидного неравенства $z \varphi^{\prime}(z)>0, z \in U$. Лемма доказана.

Перейдем к критерию устойчивой положительности, связанному с аналитическими свойствами функщий $\varphi_{i i}$. Для этого положим

$$
R^{*}(A)=\sup \left\{R_{i i}^{0}(A), i=1,2, \ldots\right\} .
$$

В силу предложения 3.2 точную верхнюю грань здесь можно заменить на максимум, причем он достигается лиш в конечном числе точек.

Ниже мы иногда используем обозначение $C(\rho)$ для круга $\{z:|z|<\rho\}, \rho>0$, на комплексной плоскости. Всякую функцию, определенную в $C(\rho)$ и аналитическую всюду за исключением, быть может, конечного числа полюсов, будем называть мероморфной в $C(\rho)$. 
3.5. ПРЕДЛОЖЕНИЕ. 1) Если неразложимая матрица А устойчиво-положительна, то при любом $i$ функиии $\varphi_{i i}(A, \cdot)$ и $\varphi_{i i}^{0}(A, \cdot)$ продолжаются до мероморфных функиий в круге $C\left(R^{*}(A)\right)$, причем существует такое $r>R(A)$ (не зависящее от $i)$, что особенности каждой из функций $\varphi_{i i}(A, \cdot)$ в круге $C(r)$ исчерпываются простыми полюсами в точках $R(A) \exp \left(2 \pi i k / d\left(G_{A}\right)\right), 0 \leqslant k \leqslant$ $d\left(G_{A}\right)-1$.

2) Если для неразложимой матрищь $A$ c $R(A)>0$ найдется такое $i$, что при некотором $r>R(A)$ функиия $\varphi_{i i}(A, \cdot)$ продолжается до мероморфной функции в круге $C(r)$, то матрица $A$ устойчиво-положстельна.

ДокАЗАТЕльство. 1) Если $A$ - устойчиво-положительная матрица, то $R(A)>0$ и в силу п. 2 леммы 2.6 и предложения 2.9 при всех $i$ вьполняется неравенство $R_{i i}^{0}(A)>$ $R(A)$. В таком случае соотношение (2.10) реализует аналитическое продолжение функции $\varphi_{i i}(A, \cdot)$ в круг $C\left(R_{i i}^{0}(A)\right)$, причем из очевидного неравенства

$$
\left.\frac{d}{d z} \varphi_{i i}^{0}(A, z)\right|_{z=R(A)}>0
$$

вытекает простота полюса в точке $R(A)$.

Теперь зафиксируем произвольное $i$, для которого $R_{i i}^{0}(A)=R^{*}(A)$ и, следовательно, функция $\varphi_{i i}^{0}(A, \cdot)$ аналитична в круге $C\left(R^{*}(A)\right)$. Воспользовавшись леммой 3 из $[21]$, можно утверждать, что при любом $j$ функция $\varphi_{j j}^{0}(A, \cdot)$ мероморфна в этом круге и $\varphi_{j j}^{0}(A, z) \neq 1$ во всех точках $z$, где $\varphi_{i i}^{0}(A, z) \neq 1$. Но тогда (см. $\left.(2.10)\right)$ и функшия $\varphi_{i i}(A, \cdot)$ мероморфна в том же круге, а в силу леммы 3.4 найдется такое $r>R(A)$, что все ее особенности в круге $C(r)$ исчерпьваются простыми полюсами в точках, указанных в формулировке (очевидно, можно положить $r=\min \{|z|:|z|>R(A)$, $\left.\left.\varphi_{i i}^{0}(A, z)=1\right\}\right)$.

2) Пусть $A$ обладает свойствами, указанными в условии. Тогда с помощью соотношения (2.10), переписанного при $j=i$ в виде

$$
\varphi_{i i}^{0}(A, z)=\varphi_{i i}(A, z)\left(1+\varphi_{i i}(A, z)\right)^{-1}, \quad|z|<R(A),
$$

функцию $\varphi_{i i}^{0}(A, \cdot)$ можно аналитически продолжить на множество $C(r) \backslash F$, где $F=\left\{z: \varphi_{i i}(A, z)=-1\right\}$.

Из (3.3) и леммы 2.6 следует, что $F$ не пересекается с кругом $C(R(A))$. Но $F$ не пересекается и с его замыканием. Действительно, вследствие мероморфности $\varphi_{i i}(A, \cdot)$ у каждой точки $z_{0} \in F \cap C(r)$ найдется окрестность, в которой эта функщия аналитична. Отсюда следует, что если $\left|z_{0}\right|=R(A)$, то $\varphi_{i i}\left(A, z_{n}\right) \rightarrow-1$ при $n \rightarrow \infty$ для любой последовательности точек $z_{n} \in C(R(A))$, сходящейся к $z_{0}$. Тогда $\left|\varphi_{i i}^{0}\left(A, z_{n}\right)\right| \rightarrow \infty$ (см. (3.3)) и, тем более, $\varphi_{i i}^{0}\left(A,\left|z_{n}\right|\right) \rightarrow \infty$. Так как $\left|z_{n}\right| \rightarrow R(A)$, последнее означает, что $\varphi_{i i}^{0}(A, R(A))=\infty$. Но это противоречит п. 2 леммы 2.6. Таким образом, не сушествует точек $z \in F$, для которых $|z| \leqslant R(A)$. Поэтому найдется такое $r_{1} \in(R(A), r)$, что $F \cap C\left(r_{1}\right)=\varnothing$, и следовательно, функция $\varphi_{i i}^{0}(A, \cdot)$ аналитична в круге $C\left(r_{1}\right)$, т.е. $R_{i i}^{0}(A) \geqslant r_{1}>R(A)$. В силу того же п. 2 леммы 2.6 отсюда вытекает устойчивая положительность матрицы $A$. Предложение доказано.

Теперь свяжем устойчивую положительность с асимптотическим поведением коэффициентов ряда $\varphi_{i i}^{0}(A, \cdot)$. Особенно просто эта связь вьглядит в случае стохастических матриц. 
3.6. ОПредЕлениЕ (ср. [23]). Назовем стохастическую матрищу $A$ геометрически әргодической, если сушествуют такие $\rho \in(0,1), c_{i j}>0, q_{j}>0(i, j \in \mathbb{N})$, что

$$
\left|Q_{i j}(A, n)-q_{j}\right| \leqslant c_{i j} \rho^{n}, \quad i, j, n \in \mathbb{N} .
$$

Следующий факт, по существу, содержится в [21].

3.7. ПРЕДЛОЖЕНИЕ. Неразложимая возвратная стохастическая матрица является геометрически әргодической в том и только том случае, когда она апериодична и устойчиво-положительна.

ДокАЗАТЕЛЬСТво. Пусть матрица $A$ апериодична и устойчиво-положительна. Из ее стохастичности и возвратности следует, что $R(A)=1$ (см. п. 1 леммы 2.6). Пользуясь предложением 3.1 , будем считать все функции $\varphi_{i i}(A, \cdot)$ определенньми в круге $C(r), r>1$, где они имеют своей единственной особенностью простой полюс в точке 1. Это значит, что при каждом $i$ функция $\psi_{i i}(z)=(z-1) \varphi_{i i}(A, z)$ аналитична в упомянутом круге. Разложив ее в ряд

$$
\psi_{i i}(z)=\sum_{n=1}^{\infty} b_{i}(n) z^{n}, \quad|z|<r,
$$

убеждаемся, что

$$
b_{i}(1)=-Q_{i i}(A, 1), \quad b_{i}(n)=Q_{i i}(A, n-1)-Q_{i i}(A, n), \quad n \geqslant 2 .
$$

В силу неравенства Коши

$$
\left|b_{i}(n)\right| \leqslant c_{i}(\rho) \rho^{-n}, \quad n \geqslant 1,
$$

где $\rho$ - любое число из интервала $(1, r)$ и $c_{i}(\rho)=\max _{|z|=\rho}\left|\psi_{i i}(z)\right|$. Но тогда

$$
\left|Q_{i i}(A, n)-q_{i}\right| \leqslant \frac{c_{i}(\rho)}{\rho-1} \rho^{-n}
$$

где $q_{i}$ - стационарные вероятности цепи Маркова с матрицей перехода $A$. Согласно [21] отсюда вытекает геометрическая эргодичность.

Обратно, пусть матрица $A$ геометрически эргодична. Очевидно, тогда она апериодична, а из ее возвратности следует, что $R(A)=1$. Повторив теперь только что проведенное рассуждение в обратном порядке, приходим к выводу, что функция $(z-1) \varphi_{i i}(A, z)$ аналитична в некотором круге $C(r)$, где $r>1$. В силу п. 2 предложения 3.5 это означает, что матрица $A$ устойчиво-положительна. Предложение доказано.

Обратимся к критерию устойчивой положительности, основанному на совершенно другой идее. Прежде всего заметим, что в случае стохастической матрищы устойчивая положительность влечет за собой весьма сильные свойства возвратности соответствуюшей цепи Маркова: частица, совершаюшая случайное блуждание, описываемое 
этой цепю, имеет тенденцию держаться вблизи своего начального положения. Доказываемая ниже теорема 3.8 утверждает, что устойчивую положительность можно интерпретировать как стремление такой частищы оставаться внутри некоторого конечного множества, содержащего точку, из которой она вьшла. В дальнейшем мы увидим, что эта теорема служит своеобразным критерием компактности для последовательности гиббсовских мер, связанных с конечными подматрицами матрицы $A$. Чтобы сформулировать ее, необходимо ввести еще несколько понятий.

Назовем подматрищей матрицы $A$ результат последовательного проведения двух операций: замены нулями произвольных элементов $a_{i j}$ и вычеркивания при каждом $i$ строки и столбца с номером $i$, если они целиком состоят из нулей (эта терминология немного отличается от общепринятой). В дальнейшем явное указание на вторую из этих операций будет, как правило, опускаться. Включение $A^{\prime} \subset A$ будет означать, что $A^{\prime}$ - подматрица матрицы $A$. Если подматрицу можно получить путем вычеркивания строк и столбцов с одинаковыми номерами, то она называется главной. Множество номеров строк (и столбцов) главной подматрицы $A^{\prime}$ будем назьвать ее носителем и обозначать $\operatorname{supp}\left(A^{\prime}\right)$. Как и в $\S 2$, поставим в соответствие матрище $A$ ориентированньй граф̆ $G_{A}$ с множеством вершин $V=V\left(G_{A}\right)$. Точно так же, всякой подматрице $A^{\prime} \subset A$ отвечает подграф $G_{A^{\prime}}$ графа $G_{A}$ (будем писать $G_{A^{\prime}} \subset G_{A}$ ), и, как нетрудно понять, переход от $A^{\prime}$ к $G_{A^{\prime}}$, рассматриваемьй лишш на множестве неразложимых подматриц, реализует взаимнооднозначное соответствие между этим множеством и множеством связных подграфов графа $G_{A}$. Подграфы $G_{A^{\prime}} \subset G_{A}$, отвечаюшие главным подматрицам $A^{\prime} \subset A$, будем называть главнылми подграфами. Очевидно, множество вершин $V\left(G_{A^{\prime}}\right)$ такого подграфа совпадает с $\operatorname{supp}\left(A^{\prime}\right)$, а его ребрами служат те и только те ребра графа $G_{A}$, которые ведут из $V\left(G_{A^{\prime}}\right)$ в $V\left(G_{A^{\prime}}\right)$.

Пусть $V^{\prime}-$ произвольное подмножество множества $V\left(G_{A}\right)=\operatorname{supp}(A)$. Для любого $n \geqslant 1$ и любых $i, j \in V$ введем множество $\Gamma_{i j}\left(G_{A}, n \mid V^{\prime}\right)$, состояшее из тех путей $\gamma \in \Gamma_{i j}\left(G_{A}, n\right)$, которые не проходят через $V^{\prime}$ (здесь не исключается, что $i, j \in V^{\prime}$, см. определение 1.1). Пусть $Q_{i j}\left(A, n \mid V^{\prime}\right)$ - сумма весов всех этих путей и

$$
\varphi_{i j}\left(A, z \mid V^{\prime}\right)=\sum_{n=1}^{\infty} Q_{i j}\left(A, n \mid V^{\prime}\right) z^{n} .
$$

Обозначив через $r_{i j}\left(A \mid V^{\prime}\right)$ радиус сходимости ряда $(3.4)$, положим

$$
r\left(A \mid V^{\prime}\right)=\inf _{i, j \in V^{\prime}} r_{i j}\left(A \mid V^{\prime}\right)
$$

3.8. ТеоремА. Для устойчивой положительности бесконечной неразложимой матриць А необходимо и достаточно, чтобь у нее нашлась конечная неразложимая главная подматрица $B$, удовлетворяющая неравенству

$$
R(B) \leqslant r(A \mid \operatorname{supp}(B)) \text {. }
$$

Для частного случая матриц, состояших из нулей и единиц, достаточность этого условия установлена в [24].

Прежде чем доказьвать теорему, введем одно новое понятие и докажем лемму, которая будет использована также в $\S 6$ этой статьи. 
3.9. ОПредЕлЕниЕ. Назовем последовательность конечных подграфов $G^{(n)}, n=$ $1,2, \ldots$, бесконечного графа $G$ исчерпьвающей, если $G^{(n)} \subset G^{(n+1)}, \bigcup_{n} V\left(G^{(n)}\right)=$ $V(G)$ и $\bigcup_{n} E\left(G^{(n)}\right)=E(G)$. Последовательность конечных подматриц $A^{(n)}$ бесконечной матрицы $A$ будем назьвать исчерпывающей, если $\left\{G_{A^{(n)}}\right\}$ - исчерпьвающая последовательность подграфов графа $G_{A}$.

3.10. ЛЕмма. 1) Всякая неразложимая неотрицательная бесконечная матрица А обладает исчерпьвающей последовательностью неразложимых главных подматрии.

2) Если $\left\{A^{(n)}\right\}-$ - юбая такая последовательность, то существует предел

$$
\lim _{n \rightarrow \infty} R\left(A^{(n)}\right)=R^{+}(A),
$$

зависящий только от $A$ (но не от $\left.\left\{A^{(n)}\right\}\right)$; если при этом матрица $A$ допустимa, mо $R^{+}(A)=R(A)$.

3) Eсли $R^{+}(A)>0$, то матрица A допустима.

ДокАЗАТЕЛЬСТво. 1) Отождествляя, как и прежде, вершины графа $G_{A}$ с натуральными числами, зафиксируем для каждой вершины $k$ произвольный цикл $\gamma_{k}$, содержаший 1 и $k$. Пусть $W_{k}$ - множество вершин, составляюших этот цикл, и $V_{n}=$ $\bigcup_{k \leqslant n} W_{k}$. Рассмотрим главный подграф̆ $G^{(n)}$ графра $G_{A}$ с $V\left(G^{(n)}\right)=V_{n}$ и возьмем в качестве $A^{(n)}$ главную подматрицу матрицы $A$, определяемую условием $\operatorname{supp}\left(A^{(n)}\right)=$ $V_{n}$. Легко видеть, что тем самьм построена исчерпывающая последовательность неразложимых главных подматриц.

2) Пусть $\left\{A^{(n)}\right\}$ - произвольная последовательность подматрищ, обладающая указанными свойствами. Теорема Перрона-Фробениуса и замечание 2.2 позволяют утверждать, что $0<R\left(A^{(n)}\right)<\infty, n=1,2, \ldots$ Из определения непосредственно вытекает, что $R\left(A^{(n)}\right)$ - невозрастающая последовательность. Поэтому сушествует предел (3.6). Взяв любую другую последовательность подматриц с теми же свойствами, легко убедиться, что этот предел не изменится. Если матрища $A$ допустима, то, очевидно, $R^{+}(A) \geqslant R(A)$. Согласно предложению 3.1 при каждом $n$ матрица $A^{(n)}$ устойчиво-положительна и, следовательно, возвратна. Поэтому для всякой вершины $i \in V_{n}$ вьполняется равенство $\varphi_{i i}^{0}\left(A^{(n)}, R\left(A^{(n)}\right)\right)=1$ (см. п. 1 леммы 2.6). Что касается матрищы $A$, то $\varphi_{i i}^{0}(A, R(A)) \leqslant 1$ (см. п. 2 леммы 2.6). Следовательно (см. (2.7)), справедливы соотношения

$$
\begin{gathered}
\sum_{\gamma \in \Gamma_{i i}^{0}\left(G_{A}\right)} Q(A, \gamma)[R(A)]^{l(\gamma)} \leqslant 1, \\
\sum_{\gamma \in \Gamma_{i i}^{0}\left(G_{A^{(n)}}\right)} Q\left(A^{(n)}, \gamma\right)\left[R\left(A^{(n)}\right)\right]^{l(\gamma)}=1 .
\end{gathered}
$$

Взяв в качестве $i$ любую вершину из $V_{1}$, можно считать $(3.7),(3.8)$ вьполненными при Bcex $n$.

Предположим, что $R^{+}(A)>R(A)$, и зафиксируем произвольную точку $r$ интервала $\left(R(A), R^{+}(A)\right)$. Тогда

$$
\sum_{\gamma \in \Gamma_{i i}^{0}\left(G_{A}\right)} Q(A, \gamma) r^{l(\gamma)}>1
$$


В самом деле, если в (3.7) достигается равенство, то (3.9) вытекает из неравенства $r>R(A)$; если же в (3.7) имеет место строгое неравенство, то в силу п. 2 леммы 2.6 $R_{i i}^{0}(A)=R(A)$, и левая часть (3.9) равна бесконечности (она может быть конечна лишь в случае, когда матрица $A$ устойчиво-положительна). С другой стороны, в силу (3.8) при любом $n$ справедливо неравенство

$$
\sum_{\gamma \in \Gamma_{i i}^{0}\left(G_{A^{(n)}}\right)} Q\left(A^{(n)}, \gamma\right) r^{l(\gamma)}<1 .
$$

Заметим теперь, что, во-первых, $Q\left(A^{(n)}, \gamma\right)=Q(A, \gamma)$ для любого пути $\gamma \in \Gamma_{i i}^{0}\left(A^{(n)}\right)$ и, во-вторых, неравенство (3.9), очевидно, останется справедливым, если в его левой части заменить множество $\Gamma_{i i}^{0}(A)$ на его достаточно большое конечное подмножество $\Gamma^{\prime}$. Но всякое такое $\Gamma^{\prime}$ будет частью множества $\Gamma_{i i}^{0}\left(A^{(n)}\right)$ при любом достаточно большом $n$. Поэтому справедливость (3.9) для $\Gamma^{\prime}$ противоречит (3.10). Тем самым равенство $R^{+}(A)=R(A)$ доказано.

3) Это утверждение составляет часть теоремы С в [25], поэтому его доказательство (достаточно простое) мы опустим.

3.11. ЗАмЕчАнИя. 1) Лемма 3.10 позволяет обобшить определение $R(A)$ на любые неразложимые неотрицательные матрищы, не заботясь об их допустимости, а именно, отождествить $R(A)$ с $R^{+}(A)$.

2 ) Для любой неотрицательной матрицы $A$ (не обязательно неразложимой) положим

$$
\lambda(A)=\sup _{A^{\prime} \subset A} \lambda\left(A^{\prime}\right)
$$

где $\lambda\left(A^{\prime}\right)$ - максимальное собственное число конечной подматрицы $A^{\prime} \subset A$ и sup берется по всем таким подматрицам. Если бесконечная матрица $A$ неразложима, то в силу леммы 3.10 для нее сохраняется соотношение $R(A)=[\lambda(A)]^{-1}$ (см. замечание 2.3).

3.12. ДоКАЗАТЕЛЬСТво ТЕОРемЫ 3.8. Начнем с установления нескольких фактов, которые будут использованы ниже как при доказательстве необходимости условия теоремы, так и при доказательстве его достаточности. Пусть $A$ - неразложимая неотрицательная бесконечная матрица, $B$ - ее конечная неразложимая главная подматрица и $W=\operatorname{supp}(B)$. Как и раншше, обозначим через $G_{B}$ главньй подграф графа $G_{A}$, определяемый равенством $V\left(G_{B}\right)=W$. Для любых $i, j, k \in W$ и $n \geqslant 1$ выделим в множестве $\Gamma_{i j}\left(G_{A}, n \mid k\right.$ ) (здесь и в аналогичных ситуациях ниже мы пишем $k$ вместо $\{k\})$ подмножество $\Gamma_{i j}\left(G_{B}, n \mid k\right)$, состояшее из всех путей $\gamma \in \Gamma_{i j}\left(G_{A}, n \mid k\right)$, являюшихся $G_{B}$-путями, и подмножество $\Gamma_{i j}\left(G_{B}, G_{A}, n \mid k\right)$, состояшее из всех путей $\gamma \in \Gamma_{i j}\left(G_{A}, n \mid k\right)$ вида $\gamma=\gamma_{1} \gamma_{2}$ и $\gamma=\gamma_{2}$, где $\gamma_{1}-G_{B}$-путь, ведуший из $i$ в какую-либо вершину $p \in W$, а $\gamma_{2}-G_{A}$-путь длины $\geqslant 2$, ведуший из $p$ в $j$ и не проходящий через $W$ (ясно, что если $\gamma=\gamma_{2}$, то $p=i$ ). Очевидно, эти подмножества не пересекаются. Пусть $Q_{i j}(B, n \mid k)$ и $Q_{i j}(B, A, n \mid k)$ - суммы весов всех путей, входяших в $\Gamma_{i j}\left(G_{B}, n \mid k\right)$ и в $\Gamma_{i j}\left(G_{B}, G_{A}, n \mid k\right)$ соответственно. Для удобства будем считать, что $Q_{i j}(B, n \mid k), Q_{i j}(B, A, n \mid k)$ определены при всех $n \in \mathbb{Z}$ и равны нулю при $n \leqslant 0$ (впрочем, из определения видно, что $Q_{i j}(A, B, n \mid k)=0$ и при $\left.n=1\right)$. Введем 
производящие функции

$$
\begin{gathered}
\varphi_{i j}(B, z \mid k)=\sum_{n=1}^{\infty} Q_{i j}(B, n \mid k) z^{k}, \\
\varphi_{i j}(B, A, z \mid k)=\sum_{n=1}^{\infty} Q_{i j}(B, A, n \mid k) z^{k}
\end{gathered}
$$

и обозначим соответственно через $r_{i j}(B \mid k), r_{i j}(B, A \mid k)$ радиусы сходимости этих степенных рядов.

Из определения чисел $Q_{i j}(B, n \mid k), Q_{i j}(B, A, n \mid k)$ и очевидного равенства

$$
Q\left(A, \gamma_{1} \gamma_{2}\right)=Q\left(A, \gamma_{1}\right) Q\left(A, \gamma_{2}\right)
$$

непосредственно вытекает, что при любых $i, j, k \in W$ и любом $n \geqslant 2$

$$
Q_{i j}(B, A, n \mid k)=Q_{i j}(A, n \mid W)+\sum_{p \in W \backslash\{k\}} \sum_{m=1}^{n-2} Q_{i p}(B, m \mid k) Q_{p j}(A, n-m \mid W)
$$

Отсюда получаем тождество

$$
\left.\varphi_{i j}(B, A, z \mid k)=\varphi_{i j}(A, z \mid W)+\sum_{p \in W \backslash\{k\}} \varphi_{i p}(B, z \mid k\}\right) \varphi_{p j}(A, z \mid W),
$$

а из него - следующее соотношение между радиусами сходимости (вытекающее из неотрицательности коэффициентов всех этих рядов):

$$
r_{i j}(B, A \mid k)=\min _{p \in W} \min \left(r_{p j}(A \mid W), r_{i p}(B \mid k)\right), \quad i, j, k \in W .
$$

Ниже нам понадобятся еще некоторые соотношения между производяшими функциями. Рассмотрим произвольный путь $\gamma$ из $\Gamma_{i j}\left(G_{A}, n \mid j\right), n \geqslant 1$. Если он не принадлежит множеству

$$
\Gamma_{i j}\left(G_{B}, n \mid j\right) \cup \Gamma_{i j}\left(G_{B}, G_{A}, n \mid j\right)
$$

то при некоторых $k \in W \backslash\{j\}, m \in[2, n-1]$ его можно однозначньм образом представить в виде $\gamma=\gamma^{\prime} \gamma^{\prime \prime}$, где

$$
\gamma^{\prime} \in \Gamma_{i k}\left(G_{B}, G_{A}, m \mid j\right), \quad \gamma^{\prime \prime} \in \Gamma_{k j}\left(G_{A}, n-m \mid j\right)
$$

Обратно, если $\gamma^{\prime}, \gamma^{\prime \prime}$ - любые пути из только что указанных множеств, то, как легко понять, $\gamma=\gamma^{\prime} \gamma^{\prime \prime}-G_{A}$-путь, принадлежащий $\Gamma_{i j}\left(G_{A}, n \mid j\right)$, но не принадлежащий $\Gamma_{i j}\left(G_{B}, n \mid j\right) \cup \Gamma_{i j}\left(G_{B}, G_{A}, n \mid j\right)$. Отсюда получаем

$$
\begin{aligned}
Q_{i j}(A, n \mid j)= & Q_{i j}(B, n \mid j)+Q_{i j}(B, A, n \mid j) \\
& +\sum_{k \in W \backslash\{j\}} \sum_{m=2}^{n-1} Q_{i k}(B, A, m \mid j) Q_{k j}(A, n-m \mid j),
\end{aligned}
$$


что приводит к тождеству

$$
\begin{aligned}
\varphi_{i j}(A, z \mid j)= & \varphi_{i j}(B, z \mid j)+\varphi_{i j}(B, A, z \mid j) \\
& +\sum_{k \in W \backslash\{j\}} \varphi_{i k}(B, A, z \mid j) \varphi_{k j}(A, z \mid j), \quad i, j \in W,
\end{aligned}
$$

справедливому для тех $z$, при которых сходятся все степенные ряды, фигурирующие в его правой части. При $i=j$ оно принимает вид

$$
\begin{aligned}
\varphi_{j j}(A, z \mid j)= & \varphi_{j j}(B, z \mid j)+\varphi_{j j}(B, A, z \mid j) \\
& +\sum_{k \in W \backslash\{j\}} \varphi_{j k}(B, A, z \mid j) \varphi_{k j}(A, z \mid j), \quad j \in W,
\end{aligned}
$$

откуда, в частности, получаем

$$
r_{j j}(A \mid j) \leqslant \min _{k \in W} r_{j k}(B, A \mid j), \quad j \in W .
$$

Перейдем непосредственно к доказательству теоремы.

Необходимость. Пусть матрища $A$ устойчиво-положительна. Согласно определению 2.8 , предложению 2.9 и п. 2 леммы 2.6 это означает, что $R_{i i}^{0}(A)>R(A)$ для любого $i$. Тогда в силу леммы 3.10 у $A$ найдется неразложимая конечная главная подматрица $B$, для которой $R(B) \leqslant R_{i i}^{0}(A)$. Пусть $G_{B}$ - отвечаюший ей главньй подграф графа $G_{A}$ и $W-$ множество его вершин, т.е. $W=\operatorname{supp}(B)$. Не ограничивая обшности, будем считать, что $i \in W$. Заметим, что по определению $r_{i i}(A \mid i)$ есть не что иное, как $R_{i i}^{0}(A)$. С учетом этого из $(3.15),(3.12)$ получаем

$$
R(B) \leqslant r_{i i}(A \mid i) \leqslant \min _{j \in W} r_{i j}(B, A \mid i) \leqslant \min _{j, p \in W} r_{p j}(A \mid W)=r(A \mid W) .
$$

Тем самым доказано, что для выбранной подматрицы $B$ вьполняется неравенство (3.5).

Достаточность. Пусть $B$ - подматрица, для которой выполняются условия теоремы. Тогда в силу (3.5)

$$
r_{p j}(A \mid W) \geqslant r(A \mid W) \geqslant R(B), \quad p, j \in W,
$$

а в силу предложения 2.1

$$
r_{i p}(B \mid k) \geqslant R_{i p}(B)=R(B), \quad i, p, k \in W
$$

откуда с помощью (3.12) получаем

$$
r_{i j}(B, A \mid k) \geqslant R(B), \quad i, j, k \in W .
$$


Теперь воспользуемся тождеством (3.14). Зафиксировав в нем произвольное $j \in W$ и беря всевозможные $i \in W$, будем рассматривать полученньй набор соотношений как систему линейных уравнений относительно неизвестных $\varphi_{i j}(A, z \mid j)$. По правилу Крамера

$$
\varphi_{i j}(A, z \mid j)=\Delta_{i j}(z) / \Delta_{j}(z), \quad i \in W,
$$

где определители $\Delta_{i j}(z)$ и $\Delta_{j}(z)$ являются многочленами от $\varphi_{i j}(B, z \mid j)$ и $\varphi_{i k}(B, A, z \mid j), i, k \in W$. Подставив (3.18) в (3.15), получим

$\varphi_{j j}(A, z \mid j)=\varphi_{j j}(B, z \mid j)+\varphi_{j j}(B, A, z \mid j)+\left[\Delta_{j}(z)\right]^{-1} \sum_{k \in W \backslash\{j\}} \Delta_{k j}(z) \varphi_{j k}(B, A, z \mid j)$.

Обозначим правую часть (3.19) через $\psi_{j}(z)$. В силу $(3.17 \mathrm{a}),(3.17 \mathrm{~b}) \psi_{j}$ представляет собой мероморфную функцию в круге $|z|<R(B)$. Предположим, что она имеет в этом круге особые точки (полюса). Тогда (3.19) вьполняется в меншшем круге, $|z|<\left|z_{0}\right|$, где $z_{0}$ - ближайший к началу координат полюс. Ясно, что в этом случае $r_{j j}(A \mid j)=$ $\left|z_{0}\right|$, а так как коэффициенты ряда $\varphi_{j j}(A, \cdot)$ неотрицательны, мы приходим к вьводу, что

$$
\varphi_{j j}\left(A,\left|z_{0}\right| \mid j\right) \geqslant\left|\varphi_{j j}\left(A, z_{0} \mid j\right)\right|=\infty .
$$

Предположим теперь, что функция $\psi_{j}$ аналитична во всем круге $|z|<R(B)$. В силу (3.19) в этом случае $r_{j j}(A \mid j) \geqslant R(B)$. Далее, из определения видно, что при всяком $n \geqslant 1$ вьполняется неравенство $Q_{j j}(A, n \mid j) \geqslant Q_{j j}(B, n \mid j)$ и что хотя бы при одном $n$ оно является строгим (поскольку граф̆ $G_{A}$ связен). Поэтому

$$
\varphi_{j j}\left(A, r_{j j}(A \mid j) \mid j\right)>\varphi_{j j}\left(B, r_{j j}(A \mid j) \mid j\right) \geqslant \varphi_{j j}(B, R(B) \mid j) .
$$

Но так как $\varphi_{j j}(B, \cdot \mid j)=\varphi_{j j}^{0}(B, \cdot)($ см. $\S 2)$, а матрица $B$ возвратна, имеет место равенство

$$
\varphi_{j j}^{0}(B, R(B))=1
$$

(см. п. 1 леммы 2.6). Подставив его в $(3.20)$, получаем $\varphi_{j j}\left(A, r_{j j}(A \mid j) \mid j\right)>1$. Таким образом, последнее неравенство выполняется в обоих случаях, т.е. матрица $A$ устойчиво-положительна. Теорема доказана.

Вьведем из теоремы 3.8 одно простое следствие, которое в дальнейшем позволит нам получить новьй критерий устойчивой положительности. Пусть, как и в этой теореме, $A$ - неразложимая бесконечная матрища, $B$ - ее неразложимая конечная главная подматрица, $W=\operatorname{supp}(B)$ и $G_{B}-$ главньй подграф̆ графа $G_{A}$, отвечающий подматрице $B$. Для любой пары различных вершин $i, j \in W$ зафиксируем кратчайший $G_{B}$-путь $\gamma_{j i}$, ведущий из $j$ в $i$ (любой из таких путей, если их несколько). Напомним, что для множества всех $G_{A}$-путей, ведуших из $i$ в $j$ и не проходяших через $W$, вьше было введено обозначение $\Gamma_{i j}\left(G_{A} \mid W\right.$ ) (относяшееся также и к случаю $i=j$ ). Пусть $\Gamma_{i j}^{\prime}\left(G_{A} \mid W\right)$ - множество путей, которое при $i=j$ совпадает с $\Gamma_{i j}\left(G_{A} \mid W\right)$, а при $i \neq j$ есть объединение $\Gamma_{i j}\left(G_{A} \mid W\right) \cup\left\{\gamma_{j i}\right\}$. Введем также множество $W_{i j}^{\prime}(A)$, 
состояшее из всех вершин графа $G_{A}$, каждая из которых принадлежит некоторому пути $\gamma \in \Gamma_{i j}^{\prime}(A \mid W)$, и множество $E_{i j}^{\prime}(A)$ ребер графа $G_{A}$, которые выделяются аналогичным условием. Подматрицу матрищы $A$, полученную из $A$ заменой нулями всех элементов $a_{m n}$, кроме тех, для которых ребро $(m, n)$ принадлежит одному из путей, входящих в $\Gamma_{i j}^{\prime}\left(G_{A} \mid W\right)$, обозначим через $A_{i j}(B)$.

3.13. СлЕДСТВИЕ. Если $R\left(A_{i j}(B)\right) \geqslant R(B)$ для любых $i, j \in W$, то матрица А устойчиво-положстельна.

ДокАЗАТЕльСТво. Пусть $i \neq j$ и $l_{j i}$ - длина пути $\gamma_{j i}$. Очевидно, при любом $n \geqslant 2$

$$
Q_{i i}\left(A_{i j}(B), n+l_{j i}\right)=Q_{i j}(A, n \mid W) Q\left(A, \gamma_{j i}\right)
$$

а потому

$$
r_{i j}(A \mid W)=r_{i i}\left(A_{i j}(B)\right) \geqslant R\left(A_{i j}(B)\right),
$$

и неравенство $R\left(A_{i j}(B)\right) \geqslant R(B)$ приводит к выводу, что $R(B) \leqslant r_{i j}(A \mid W)$, если $i \neq j$; при $i=j$ это тем более верно. Но тогда выполнено условие (3.5) и по теореме 3.8 матрица $A$ устойчиво-положительна, что и требовалось доказать.

Согласно теореме 3.8 наличие или отсутствие свойства устойчивой положительности у матрицы $A$ полностью определяется соотношением между "внутренними" параметрами $R(B)$ ее неразложимых конечных подматриц $B$ и их “внешними” параметрами $r(A \mid \operatorname{supp}(B))$. Сейчас для нас будет важно выяснить, как эти параметры изменяются при переходе от подматрицы $B$ к ее неразложимой подматрице $B^{\prime}$. Что касается внутренних параметров, то из теории неотрицательных матриц хорошо известно, что $R\left(B^{\prime}\right) \geqslant R(B)$ (см. [18, гл. 12]) (это также вытекает из того, что $-\ln R(B)$ - топологическое давление, см. $\S 4$ ). Следуюшая лемма утверждает, что между внешними параметрами имеет место обратное соотношение.

3.14. ЛЕммА. Если В, $B_{1}$ - неразложимые конечные главные подматрицы неразложимой бесконечной матрицы $A$, причем $B_{1} \subset B$, то

$$
r\left(A \mid \operatorname{supp}\left(B_{1}\right)\right) \leqslant r(A \mid \operatorname{supp}(B)) \text {. }
$$

ДокАЗАТЕЛЬСтво. Пусть $W=\operatorname{supp}(B), W_{1}=\operatorname{supp}\left(B_{1}\right)$. Каждой вершине $k \in W$ следуюшим образом поставим в соответствие вершины $k^{\prime}, k^{\prime \prime} \in W_{1}$ : если $k \in W_{1}$, то $k^{\prime}=k^{\prime \prime}=k$; если $k \in W \backslash W_{1}$, то в качестве $k^{\prime}$ возьмем произвольную вершину $k_{1} \in W_{1}$, из которой ведет кратчайший путь в $k$, а в качестве $k^{\prime \prime}$ - произвольную вершину $k_{1} \in W_{1}$, в которую ведет кратчайший путь из $k$. Пусть $\gamma_{k^{\prime} k}-$ первьй, а $\gamma_{k k^{\prime \prime}}-$ второй из упомянутых путей (в обоих случаях его можно выбирать произвольно, если таких путей несколько).

Поставим в соответствие каждому пути $\gamma \in \Gamma_{i j}\left(G_{A}, n \mid W\right)$, где $i, j \in W, n \geqslant 2$, путь

$$
\gamma_{1}= \begin{cases}\gamma, & \text { если } i, j \in W_{1}, \\ \gamma_{i^{\prime} i} \gamma, & \text { если } i \in W \backslash W_{1}, j \in W_{1}, \\ \gamma \gamma_{j j^{\prime \prime}}, & \text { если } i \in W_{1}, j \in W \backslash W_{1}, \\ \gamma_{i^{\prime} i} \gamma \gamma_{j j^{\prime \prime}}, & \text { если } i, j \in W \backslash W_{1} .\end{cases}
$$


В каждом из фигурируюших в правой части этого равенства четырех случаев введем величины $l_{i}^{\prime}, a_{i}^{\prime}, l_{j}^{\prime \prime}, a_{j}^{\prime \prime}$, положив $l_{i}^{\prime}=l_{j}^{\prime \prime}=0, a_{i}^{\prime}=a_{j}^{\prime \prime}=1$ в первом случае, $l_{i}^{\prime}=l\left(\gamma_{i^{\prime} i}\right)$, $a_{i}^{\prime}=Q\left(A, \gamma_{i^{\prime} i}\right), l_{j}^{\prime \prime}=0, a_{j}^{\prime \prime}=1$ - во втором, $l_{i}^{\prime}=0, a_{i}^{\prime}=1, l_{j}^{\prime \prime}=l\left(\gamma_{j j^{\prime \prime}}\right), a_{j}^{\prime \prime}=Q\left(A, \gamma_{j j^{\prime \prime}}\right)$ - в третьем и $l_{i}^{\prime}=l\left(\gamma_{i^{\prime} i}\right), a_{i}^{\prime}=Q\left(A, \gamma_{i^{\prime} i}\right), l_{i}^{\prime \prime}=l\left(\gamma_{j j^{\prime \prime}}\right), a_{j}^{\prime \prime}=Q\left(A, \gamma_{j j^{\prime \prime}}\right)$ - в четвертом. Очевидно,

$$
l\left(\gamma_{1}\right)=l_{i}^{\prime}+n+l_{j}^{\prime \prime}, \quad Q\left(A, \gamma_{1}\right)=a_{i}^{\prime} Q(A, \gamma) a_{j}^{\prime \prime},
$$

причем величины $l_{i}^{\prime}, a_{i}^{\prime}, l_{j}^{\prime \prime}, a_{j}^{\prime \prime}$ не зависят от $\gamma($ а зависят лишь от $i, j)$ и $\gamma_{1} \in \Gamma_{i^{\prime} j^{\prime \prime}}\left(G_{A}\right.$, $\left.l_{i}^{\prime}+n+l_{j}^{\prime \prime} \mid W_{1}\right)$. Так как из разных $\gamma$ получаются разные $\gamma_{1}$, мы приходим к неравенству

$$
Q_{i j}(A, n \mid W) \leqslant\left(a_{i}^{\prime} a_{j}^{\prime \prime}\right)^{-1} Q_{i^{\prime} j^{\prime \prime}}\left(A, l_{i}^{\prime}+n+l_{j}^{\prime \prime} \mid W_{1}\right),
$$

из которого видно, что

$$
r_{i j}(A \mid W) \geqslant r_{i^{\prime} j^{\prime \prime}}\left(A \mid W_{1}\right) \geqslant r\left(A \mid W_{1}\right),
$$

и, следовательно, вьполняется (3.22).

Теперь мы можем вьвести из теоремы 3.8 последний в этом параграфе критерий устойчивой положительности. Он формулируется очень коротко.

3.15. ТЕОРемА. Для устойчивой положстельности неразложсимой неотрицательной матричь А необходимо и достаточно, чтобы любая ее неразложимая собственная подматрица $A^{\prime}$ удовлетворяла неравенству $R\left(A^{\prime}\right)>R(A)$.

Для частного случая матриц, состоящих из нулей и единиц, эта теорема (в другой, но эквивалентной формулировке) имеется в [17]. Приведенное там доказательство можно перенести и на общий случай. Мы, однако, вместо этого воспользуемся теоремой 3.8 .

ДокАЗАТЕльство. Необходимость. Пусть $A^{\prime}$ - произвольная неразложимая собственная подматрица неразложимой устойчиво-положительной матрицы $A$ и $\left(i^{\prime}, j^{\prime}\right)-$ ребро графа $G_{A}$, которое не является ребром графа $G_{A^{\prime}}$. При любом $\varepsilon \in(0,1)$ введем матрищу $A^{\varepsilon}=A^{\varepsilon}\left(i^{\prime}, j^{\prime}\right)=\left(a_{i j}^{\varepsilon}\right)$, положив $a_{i j}^{\varepsilon}=a_{i j}$ при $(i, j) \neq\left(i^{\prime}, j^{\prime}\right), a_{i^{\prime} j^{\prime}}^{\varepsilon}=$ $(1-\varepsilon) a_{i^{\prime} j^{\prime}}$. Очевидно, $G_{A^{\varepsilon}}=G_{A}, R_{i^{\prime} i^{\prime}}^{0}\left(A^{\varepsilon}\right)=R_{i^{\prime} i^{\prime}}^{0}(A)$ и найдется такое $\varepsilon_{0}>0$, что если $\varepsilon<\varepsilon_{0}$, то $\varphi_{i^{\prime} i^{\prime}}^{0}\left(A^{\varepsilon}, R_{i^{\prime} i^{\prime}}^{0}\left(A^{\varepsilon}\right)\right)>1$ (так как по условию $\varphi_{i^{\prime} i^{\prime}}^{0}\left(A, R_{i^{\prime} i^{\prime}}^{0}(A)\right)>1$, см. определение 2.8 ), т.е. $A^{\varepsilon}$ (как и $A$ ) - устойчиво-положительная матрица. Но тогда (см. п. 1 леммы 2.6)

$$
\varphi_{i^{\prime} i^{\prime}}^{0}\left(A^{\varepsilon}, R\left(A^{\varepsilon}\right)\right)=\varphi_{i^{\prime} i^{\prime}}^{0}(A, R(A))=1, \quad \varepsilon<\varepsilon_{0} .
$$

$\mathrm{C}$ другой стороны, так как по условию $a_{i^{\prime} j^{\prime}}>0$, из определения $A^{\varepsilon}$ видно, что $\varphi_{i^{\prime} i^{\prime}}^{0}\left(A^{\varepsilon}, x\right)<\varphi_{i^{\prime} i^{\prime}}^{0}(A, x)$ при любом $x \in\left(0, R_{i i}^{0}(A)\right)$. Поэтому (3.22) может вьполняться лишь при $R\left(A^{\varepsilon}\right)>R(A)$.

Переписав последнее неравенство в виде $\lambda\left(A^{\varepsilon}\right)<\lambda(A)$ (см. замечание 3.11), остается заметить, что $A^{\prime} \subset A^{\varepsilon}$ при любом $\varepsilon \in(0,1)$ и, следовательно, $\lambda\left(A^{\prime}\right) \leqslant \lambda\left(A^{\varepsilon}\right)$. Беря $\varepsilon<\varepsilon_{0}$, получаем $\lambda\left(A^{\prime}\right)<\lambda(A)$, т.е. $R\left(A^{\prime}\right)>R(A)$.

Достаточность. Пусть $\left\{A^{(n)}\right\}$ - исчерпьвающая последовательность неразложимых главных подматриц рассматриваемой матрицы $A$ (см. лемму 3.10$)$. Положив 
$W^{(n)}=\operatorname{supp}\left(A^{(n)}\right)$, построим при каждом $n$ для всех пар вершин $i, j \in W^{(n)}$ подматрицы $A_{i j}\left(A^{(n)}\right)$, о которых говорилось в следствии 3.13 . Если $n$ достаточно велико, то все они будут собственньми подматрицами. Действительно, в противном случае при сколь угодно большом $n$ найдутся такие $i, j \in W^{(n)}$, что кратчайший $G_{A^{(n)} \text {-путь }}$ $\gamma_{j i}$, ведуший из $j$ в $i$, проходит через все ребра этого графа. Но тогда в $G_{A^{(n)}}$ из каждой вершины выходит не более одного ребра (путь $\gamma_{j i}$, будучи кратчайшим, проходит через каждую вершину не более одного раза), что при достаточно большом $n$ невозможно вследствие связности $A$ и свойств последовательности $\left\{A^{(n)}\right\}$.

Пользуясь доказанным, подберем такое $n_{0}$, что при $n \geqslant n_{0}$ все подматрицы $A_{i j}\left(A^{(n)}\right)$ - собственные. По предположению, при всех $n \geqslant n_{0}, i, j \in W^{(n)}$ вьполняется неравенство $R\left(A_{i j}\left(A^{(n)}\right)\right)>R(A)$ и, значит (см. $\left.(3.21)\right), r_{i j}\left(A \mid W^{(n)}\right)>$ $R(A)$ при тех же $n, i, j$, т.е.

$$
r\left(A \mid W^{(n)}\right)>R(A), \quad n \geqslant n_{0} .
$$

Теперь с помошью лемм 3.10 и 3.14 можно подобрать такое $n \geqslant n_{0}$, что

$$
r\left(A \mid W^{(n)}\right)>R\left(A^{(n)}\right),
$$

т.е. для подматрицы $B=A^{(n)}$ выполняются условия теоремы 3.8. По этой теореме матрица $A$ устойчиво-положительна.

3.16. ЗАмечАниЕ. Из доказательства теоремы 3.15 видно, что в ее формулировке можно заменить неравенство $R\left(A^{\prime}\right)>R(A)$ на $\lambda\left(A^{\prime}\right)<\lambda(A)$, а также отказаться от требования неразложимости матрицы $A^{\prime}$. Это, очевидно, усиливает утверждение о необходимости рассматриваемого условия для устойчивой положительности $A$. Другое необходимое и достаточное условие устойчивой положительности матрицы $A$, по сушеству в явном виде содержашееся в доказательстве (см. его первую часть "необходимость"), состоит в том, что в результате умножения произвольного ее положительного элемента $a_{i^{\prime} j^{\prime}}$ на число $(1-\varepsilon)$, где $0<\varepsilon \leqslant 1$, получается матрица $A^{\varepsilon}=A^{\varepsilon}\left(i^{\prime}, j^{\prime}\right)$, для которой $R\left(A^{\varepsilon}\right)>R(A)$. При этом справедливость последнего неравенства для одной какой-нибудь пары $\left(i^{\prime}, j^{\prime}\right)$ влечет выполнение его для любой такой пары.

\section{$\S$ 4. Равновесные и гиббсовские меры. Вариационный принцип Гиббса}

Продолжим начатую в $\S 1$ тему, связанную с понятием топологического давления и вариационньм принципом. Как и там, будем рассматривать СЦМ $(X(G), S)$, где $G$ - связньй ориентированньй граф с множеством вершин $V(G)$ и множеством ребер $E(G) \subseteq V(G) \times V(G)$, а $S$ - сдвиг на пространстве $X(G)$ его двусторонне-бесконечных путей.

Предположим, что задана функция $f \in \mathscr{F}_{0}^{1}(G)$ (см. определение 1.10). В таком случае

$$
f(x)=-U\left(x_{0}, x_{1}\right),
$$


где $U$ - функция, определенная на $E(G)$. Продолжим $U$ с $E(G)$ на все прямое произведение $V(G) \times V(G)$, положив $U\left(v^{\prime}, v^{\prime \prime}\right)=+\infty$, если $\left(v^{\prime}, v^{\prime \prime}\right) \notin E(G)$. Как и в $\S 2$, введем функцию

$$
A_{f}\left(v, v^{\prime}\right)=\exp \left[-U\left(v^{\prime}, v^{\prime \prime}\right)\right], \quad v^{\prime}, v^{\prime \prime} \in V(G)
$$

которую (занумеровав произвольным образом вершины $v \in V(G)$ ), можно считать бесконечной квадратной матрицей. Элементы этой матрицы неотрицательны, и в случае, когда $f \equiv 0$, она состоит из нулей и единиц и совпадает с матрицей смежности графра $G$. Будем называть $A=A_{f}$ матрицей Больимана, отвечаюшей функции $f$, а также матрицей Больцмана, отвечающей потенииалу $U$. При этом, имея в виду (4.1), будем на равных правах с $A_{f}$ употреблять обозначение $Q(U)$. Очевидно, $G_{Q(U)}=G$ и, значит, связность $G$ равносильна неразложимости $Q(U)$.

Допустим сначала, что граф $G$ конечен и, следовательно, пространство $X(G)$ компактно. В этом случае все варианты топологического давления из $\S 1$ совпадают и для функшии $f \in \mathscr{F}_{0}^{1}(G)$ дают одну и ту же величину, равную $-\ln R(A)=\ln \lambda(A)$ (см. замечание 2.3), удовлетворяющую вариационному принципу (1.5). Впервые этот результат был получен В. Пэрри [9] для случая $f \equiv 0$, а затем обобщен $\Phi$. Спитцером [26] (см. также [27]). В упомянутых работах было также показано, что существует единственное равновесное распределение, которое представляет собой марковскую меру на $X(G)$ с вероятностями перехода $p_{i j}$ и одномерными вероятностями $\pi_{i}$ вида

$$
p_{i j}=a_{i j} y_{j}^{\prime} / \lambda y_{i}^{\prime}, \quad \pi_{i}=y_{i}^{\prime} y_{i}^{\prime \prime}, \quad i, j=1,2, \ldots
$$

где $\left(y_{i}^{\prime}, i=1,2, \ldots\right)=y^{\prime}$ и $\left(y_{i}^{\prime \prime}, i=1,2, \ldots\right)=y^{\prime \prime}$ - векторы, фигурируюшие в теореме 2.7 , и $\lambda=1 / R(A)$.

4.1. ЗАмЕчАниЕ. В формуле (4.1), а также в определении матрищы Больцмана знак "минус" перед $U$ выбран ради примирения двух сложившихся традиций - записи вариационного принципа в виде (1.5) (с “плюсом" в правой части), принятой в эргодической теории, и традиционного для статистической физики “минуса" в выражении для гиббсовской меры [27].

Откажемся теперь от условия конечности графа $G$. Наша первая цель - сформулировать вариащионньй принщип для топологического давления $P^{\operatorname{int}}(X(G), S, f)$. Здесь нельзя воспользоваться ни следствием 1.7 , ни даже равенством (1.5) в качестве определения, так как мы не накладьваем на функцию $f$ условия ограниченности, без которого $h_{\mu}(S)$ и $\int f d \mu$ могут одновременно принять бесконечные значения разных знаков. Поэтому необходима регуляризация правой части (1.5), которую мы проведем, следуя [25].

Прежде всего напомним несколько определений, связанных с понятием энтропии сохраняюшего меру преобразования (подробности см. в [28]-[30]). Пусть $(X, \mathscr{B}, \mu)-$ вероятностное пространство и $T: X \rightarrow X$ - преобразование, сохраняюшее $\mu$. Энтропией конечного измеримого разбиения $\alpha=\left(A_{1}, \ldots, A_{s}\right)$ пространства $X$ назьвается число

$$
H_{\mu}(\alpha)=-\sum_{n=1}^{s} \mu\left(A_{n}\right) \ln \mu\left(A_{n}\right)
$$


(при этом считается, что $0 \ln 0=0$ ). Для любого $\alpha$ сушествует предел

$$
h_{\mu}(T, \alpha)=\lim _{n \rightarrow \infty} \frac{1}{n} H_{\mu}\left(\alpha_{0}^{n}\right)
$$

где $\alpha_{0}^{n}$ - разбиение, элементы которого имеют вид $A_{i_{0}} \cap T^{-1} A_{i_{1}} \cap \cdots \cap T^{-n+1} A_{i_{n-1}}$, $1 \leqslant i_{0}, \ldots, i_{n-1} \leqslant s$. Энтропия $h_{\mu}(T)$ преобразования $T$ определяется равенством $h_{\mu}(T)=\sup _{\alpha} h_{\mu}(T, \alpha)$.

Заметим, далее, что в рассматриваемом случае

$$
P^{\text {int }}(X(G), S, f)=-\ln R(A) ;
$$

это вытекает из равенства (1.15), полученного в ходе доказательства следствия 1.7 (условия, наложенные в формулировке этого следствия на $f$, в $(1.15)$ не используются).

Пусть теперь в соответствии с обозначениями, введенными в $\S 1, \mathscr{M}(X(G), S)$ - совокупность $S$-инвариантных борелевских вероятностных мер на $X(G)$. Для любого $i \in V(G)$ обозначим через $X_{i}(G)$ множество последовательностей $x=\left(x_{n}, n \in \mathbb{Z}\right) \in$ $X(G)$, для которых $x_{n}=i$ при бесконечно многих $n<0$ и бесконечно многих $n>0$. Очевидно, это множество $S$-инвариантно. Для любого пути $\gamma \in \Gamma_{i i}^{0}(G)$ и любого $k \in[0, l(\gamma)-1]$ (по поводу обозначений см. $\S \S 1,2)$ положим

$$
X_{i}(G, \gamma, k)=X_{i}(G) \cap\left\{x \in X(G):\left(x_{-k}, x_{-k+1}, \ldots, x_{-k+l(\gamma)}\right)=\gamma\right\} .
$$

Ясно, что при любом $i \in V(G)$ множества $X_{i}(G, \gamma, k)$ разбивают $X_{i}(G)$ на счетное число борелевских подмножеств. Обозначим это разбиение через $\alpha_{i}$. Занумеровав произвольным образом циклы, составляющие $\Gamma_{i i}^{0}(G)$, т.е. представив их в виде последовательности $\gamma_{1}, \gamma_{2}, \ldots$, обозначим через $X_{i}(G, N), N=1,2, \ldots$, объединение множеств $X_{i}\left(G, \gamma_{n}, k\right)$ по всем парам $(n, k)$, в которых $1 \leqslant n \leqslant N, 0 \leqslant k \leqslant l\left(\gamma_{n}\right)-1$. При каждом $N \geqslant 1$ определим новое, на этот раз конечное разбиение $\alpha_{i}(N)$ множества $X_{i}(G)$, получающееся из $\alpha_{i}$ в результате объединения в один элемент всех $X_{i}\left(G, \gamma_{n}, k\right)$, не попавших в $X_{i}(G, N)$.

Пусть $\mathscr{E}(X(G), S)$ - множество тех мер $\mu \in \mathscr{M}(X(G), S)$, относительно которых сдвиг $S$ эргодичен (их назьвают эргодическими мерами). Для любых $i \in V(G)$, $\mu \in \mathscr{E}(X(G), S)$ положим

$V_{\mu}=\left\{j \in V(G): \mu\left(X_{j}(G)\right)=1\right\}, \quad \mathscr{E}_{i}(X(G), S)=\left\{\nu \in \mathscr{E}(X(G), S): \nu\left(X_{i}(G)\right)=1\right\}$

(заметим, что вследствие эргодичности $\mu$ и $S$-инвариантности $X_{i}(G)$ имеет место альтернатива: либо $\mu\left(X_{i}(G)\right)=1$, либо $\left.\mu\left(X_{i}(G)\right)=0\right)$. Для $f \in \mathscr{F}_{0}^{1}(G), \mu \in \mathscr{E}(X(G), S)$, $i \in V_{\mu}$ положим

$$
P_{i}(X(G), f, \mu)=\varlimsup_{N \rightarrow \infty}\left[h_{\mu}\left(S, \alpha_{i}(N)\right)+\int_{X_{i}(G, N)} f d \mu\right]
$$

Равенство

$$
P(X(G), f, \mu)=\inf _{i \in V_{\mu}} P_{i}(X(G), f, \mu)
$$


определяет на $\mathscr{E}(X(G), S)$ функционал $P(X(G), f, \cdot)$ со значениями в $\{-\infty\} \cup \mathbb{R} \cup$ $\{+\infty\}$. Будем назьвать $P(X(G), f, \mu)$ давлением в состоянии $\mu$.

Это определение требует некоторого оправдания - прежде всего потому, что $P_{i}(X(G), f, \mu)$ определяется для фиксированной нумерации путей $\gamma \in \Gamma_{i i}^{0}(G)$, зависимость от которой, разумеется, нежелательна. К счастью, на самом деле такой зависимости нет.

4.2. ПРеДЛОЖенИЕ [25, теорема А]. Пусть $A=A_{f}$ - матрица Больцмана, отвечающая функиии $f \in \mathscr{F}_{0}^{1}(G)$, где $G$ - связный ориентированный граф (конечный или бесконечный). Тогда при $R(A)>0$ для любых $\mu \in \mathscr{E}(X(G), S), i \in V_{\mu}$ давление $P_{i}(X(G), f, \mu)$ не зависит от выбранной на $\Gamma_{i i}^{0}(G)$ нумерации и верхний предел в (4.3) можно заменить пределом.

Покажем теперь, что в случае, ког да регуляризацию проводить не обязательно, она действительно не дает ничего нового.

4.3. ПРЕДЛОЖЕНИЕ. Если в условиях предложения 4.2 мера $\mu \in \mathscr{E}(X(G), S)$ такова, что $f \in L_{1}(\mu)$, то при всех $i \in V_{\mu}$ имеет место равенство

$$
P_{i}(X(G), f, \mu)=h_{\mu}(S)+\int_{X(G)} f d \mu .
$$

ДокАЗАТЕЛЬСтво. Достаточно заметить, что в силу известных теорем эргодической теории (см., например, $[29$, теорема 7.3$]$ или $[30$, п. 9.5])

$$
\lim _{N \rightarrow \infty} h_{\mu}\left(S, \alpha_{i}(N)\right)=h_{\mu}(S) \geqslant 0
$$

и что при $f \in L_{1}(\mu)$

$$
\lim _{N \rightarrow \infty} \int_{X_{i}(G, N)} f d \mu=\int_{X(G)} f d \mu
$$

$\left(\right.$ так как $\mu\left(X_{i}(G, N)\right) \rightarrow \mu\left(X_{i}(G)\right)$ при $N \rightarrow \infty$ и $f \in L_{1}(\mu)$, последний предел существует, хотя и может равняться $+\infty)$.

Теперь сформулируем вариационньй принцип для топологического давления.

4.4. Теорема [25, теорема В, замечание 3.3]. В условиях предложения 4.2 справедливо соотношение

$$
P^{\text {int }}(X(G), S, f)=-\ln R(A)
$$

если, кроме того, $R(A)>0$, то

$$
\sup _{\mu \in \mathscr{E}_{i}(X(G), S)} P_{i}(X(G), f, \mu)=\sup _{\mu \in \mathscr{E}(X(G), S)} P(X(G), f, \mu)=P^{\mathrm{int}}(X(G), S, f) .
$$


4.5. ЗАмЕчАНИЕ. Функционал $P(X(G), f, \cdot)$ определяется формулами (4.3) и (4.4) лишь на множестве эргодических мер. Было бы желательно продолжить его на всю совокупность $S$-инвариантных мер $\mathscr{M}(X(G), S)$. Разумеется, в случае, когда $\inf _{x \in X(G)} f(x)>-\infty$, это можно сделать с помошю равенства (4.5), и полученньй функционал будет обладать следующим свойством линейности [30]: если $\mu \in$ $\mathscr{M}(X(G), S)$ и $\left\{\mu_{c}\right\}$ - разложение меры $\mu$ на эргодические компоненты (отвечаюшее разложению на эргодические компоненты динамической системы $(X(G), S, \mu))$, то

$$
P(X(G), f, \mu)=\int_{X^{\prime}(G, \mu)} P\left(X(G), f, \mu_{c}\right) \mu^{\prime}(d c),
$$

где $\mu^{\prime}$ - мера, которую $\mu$ индуцирует на факторпространстве $X^{\prime}(G, \mu)$ пространства $(X(G), \mu)$, отвечаюшем разбиению на эргодические компоненты. В случае, когда $R(A)>0$ и $\mu$-произвольная мера из $\mathscr{M}(X(G), S)$, правую часть этого соотношения можно принять за определение его левой части, пользуясь тем, что в силу теоремы 4.4 подынтегральная функция ограничена. При этом, очевидно, будет вьполняться равенство

$$
\sup _{\mu \in \mathscr{M}(X(G), S)} P(X(G), f, \mu)=-\ln R(A) .
$$

Поскольку, однако, не существует эффективных методов разложения произвольной инвариантной меры на эргодические компоненты, мы оставим множество $\mathscr{E}(X(G), S)$ в качестве естественной области определения функционала $P(X(G), f, \cdot)$.

В соответствии с $\S 1$ назовем равновесной относительно $f$ (или $f$-равновесной) всякую меру $\mu \in \mathscr{E}(X(G), S)$, для которой $P(X(G), f, \mu)=P^{\operatorname{int}}(X(G), S, f)$.

4.6. ТЕОРема [25, теорема C]. В условиях предложсения 4.2 для существования $f$-равновесной мерь необходимо и достаточно, чтобы матрица $A_{f}$ была положительно-возвратной. Если $f$-равновесная мера существует, то она единственна и является марковской мерой на $X(G)$ с одномерным распределением и переходными вероятностями, определяемыми соотношениями (4.2).

В дальнейшем для $f$-равновесной меры будем употреблять обозначение $\mu^{f}$ (здесь, как и в некоторых других обозначениях, встречаюшихся ниже, отсутствует явное указание на граф̆ $G$, поскольку это не может вызвать недоразумений).

Таким образом, для бесконечных матриц Больцмана, которые положительновозвратны, ситуация с равновесньми состояниями точно такая же, как для конечных матриц Больцмана, а если условие положительной возвратности нарушается, то равновесных состояний нет.

Собственные векторы $y^{\prime}, y^{\prime \prime}$ матрищы $A$, фигурирующие в формуле $(4.2)$ и теореме 2.7, можно указать до некоторой степени явно ( $A$ может быть при этом как конечной, так и бесконечной матрицей, нужно лишь, чтобы она была неразложима и положительно-возвратна). С этой целью введем еще несколько обозначений (см. также $\S \S 1,2)$.

Пусть $G$ - произвольный ориентированньй граф. Для любых $i, j \in V(G)$ обозначим через $\Gamma_{i j}^{-}(G)$ (соответственно через $\left.\Gamma_{i j}^{+}(G)\right)$ множество путей $\gamma \in G$, которые ведут из $i$ в $j$ и не проходят через $i$ (соответственно через $j$ ). Очевидно, $\Gamma_{i j}^{+}(G)=\Gamma_{i j}^{0}(G)$. 
В условиях предложения 4.2 положим

$$
D_{i}^{0}(A, t)=\sum_{\gamma \in \Gamma_{i i}^{0}(G)} l(\gamma) Q(A, \gamma) t^{l(\gamma)-1}, \quad i \in V(G), \quad 0<t \leqslant R_{i i}^{0}(A) .
$$

Легко понять, что если $t<R_{i i}^{0}(A)$, то

$$
D_{i}^{0}(A, t)=\left.\frac{d}{d \tau} \varphi_{i i}^{0}(A, \tau)\right|_{\tau=t}
$$

(см. (2.6)), а если $t=R_{i i}^{0}(A)$, то $D_{i}^{0}(A, t)=\lim _{\tau \rightarrow t-0} D_{i}^{0}(A, \tau)$. В дальнейшем важную роль будет играть величина

$$
D_{i}(A)=D_{i}^{0}(A, R(A))
$$

С помощю формулы (2.10) легко проверить, что если матрища $A$ устойчиво-положительна (в частности, если она конечна, см. предложение 3.1 ), то

$$
-1 / D_{i}(A)=\operatorname{Res}\left(\varphi_{i i}(A, \cdot), R(A)\right),
$$

где $\operatorname{Res}(g, z)$ - вычет функции $g$ в точке $z$, являюшейся ее полюсом (см. предложение 3.5$)$.

Следующий факт также имеется в [25], хотя и не выделен там в отдельное утверждение.

4.7. ПРЕДЛОЖЕНИЕ. Если в условиях предложсения 4.2 матрица $A=A_{f}$ положительно-возвратна, то векторы $y^{\prime}(A)=\left(y_{i}^{\prime}(A), i \in V(G)\right)$ и $y^{\prime \prime}(A)=$ $\left(y_{i}^{\prime \prime}(A), i \in V(G)\right)$ можсно определить равенствами

$$
\begin{aligned}
y_{i}^{\prime}(A) & =\sum_{\gamma \in \Gamma_{i v}^{+}(G)} Q(A, \gamma)(R(A))^{l(\gamma)-1} / D_{v}(A), \\
y_{i}^{\prime \prime}(A) & =\sum_{\gamma \in \Gamma_{v i}^{-}(G)} Q(A, \gamma)(R(A))^{l(\gamma)},
\end{aligned}
$$

где $v$ - произвольная вершина графа $G$.

4.8. ЗАмЕчАнИЯ. 1. Вследствие положительной возвратности $A$ и связности $G$ все $y_{i}^{\prime}(A)$ и $y_{i}^{\prime \prime}(A)$ положительны. Тем самьм, $f$-равновесная мера, если она существует, положительна на всех открытых множествах, откуда видно, что эта мера (будучи марковской) принадлежит $\mathscr{E}_{i}(X(G), S)$ при каждом $i \in V(G)$.

2. Так как $\Gamma_{v v}^{+}(G)=\Gamma_{v v}^{-}(G)=\Gamma_{v v}^{0}(G)$, имеют место равенства

$$
R(A) D_{v}(A) y_{v}^{\prime}(A)=\varphi_{v v}^{0}(A, R(A))=1, \quad y_{v}^{\prime \prime}(A)=1
$$

(см. теорему 2.7), а поскольку собственные векторы (строка и столбец) матрищы $A$ с собственньм значением $1 / R(A)$ определены однозначно с точностью до множителя, из (4.11) видно, что векторы $y^{\prime}(A)$ и $y^{\prime \prime}(A)$, заданные равенствами $(4.9),(4.10)$, 
определяются условием, что их компоненты с индексом $v$ соответственно равны $\left(R(A) D_{v}(A)\right)^{-1}$ и единице. Из (4.11), (4.2) также видно, что

$$
\pi_{v}=\mu^{f}\left(\left\{x \in X(G): x_{0}=v\right\}\right)=\frac{1}{R(A) D_{v}(A)}, \quad v \in V(G) .
$$

В дальнейшем будем явно указьвать зависимость от $v$ и писать $y_{i}^{\prime}(A, v), y_{i}^{\prime \prime}(A, v)$, $y^{\prime}(A, v)$ и $y^{\prime \prime}(A, v)$ вместо $y_{i}^{\prime}(A), y_{i}^{\prime \prime}(A), y^{\prime}(A)$ и $y^{\prime \prime}(A)$. Заметим, с другой стороны, что вследствие единственности равновесной меры отношения $y_{j}^{\prime}(A, v) / y_{i}^{\prime}(A, v)$ и произведения $y_{i}^{\prime}(A, v) y_{i}^{\prime \prime}(A, v)$ от $v$ уже не зависят.

Перейдем теперь к рассмотрению гиббсовских мер. С точки зрения статистической физики (см., например, [5], [45]), речь будет идти об одномерной решетчатой модели со счетным спиновым пространством и взаимодействием ближайших соседей, задаваемым потенциалом $U$ со значениями в $\mathbb{R} \cup\{+\infty\}$.

Гиббсовская мера с потенциалом $U$ определяется требованием, чтобы отвечающее ей условное распределение в точках любого конечного подмножества решетки при условии, что заданы значения спиновой переменной вне этого множества, имело предписьваемьй потенциалом явный вид. Как хорошо известно, этот вид наследуется подмножествами, а потому достаточно требовать, чтобы условие, определяющее гиббсовскую меру, выполнялось для всех кубов (в одномерном случае - отрезков). В соответствии с этим сформулируем следуюшее определение, приспособленное к рассматриваемому случаю. Поскольку мы всюду рассматриваем лишь $S$-инвариантные меры, в этом определении идет речь о мерах из $\mathscr{M}(X(G), S)$ (в общем случае условие $S$-инвариантности не накладывается).

4.9. ОПРЕДЕЛЕНИЕ. Вероятностная мера $\mu \in \mathscr{M}(X(G), S)$ называется гиббсовской мерой с потенциалом $U$, если при всех $n \in \mathbb{Z}$ и всех целых $k$ вьполняются два условия:

1) сумма

$$
\begin{aligned}
& Z_{n}^{n+k}\left(x_{n}, x_{n+k}\right) \\
& =\sum_{i_{n+1}, \ldots, i_{n+k-1} \in V(G)} \exp \left[-U\left(x_{n}, i_{n+1}\right)-U\left(i_{n+k-1}, x_{n+k}\right)\right. \\
& \left.\quad-\sum_{n+1 \leqslant r \leqslant n+k-2} U\left(i_{r}, i_{r+1}\right)\right], \quad x=\left(x_{r}, r \in \mathbb{Z}\right) \in X(G),
\end{aligned}
$$

называемая статистической суммой, при $\mu$-почти всех $x$ конечна;

2) произведение

$$
\begin{aligned}
& \mu_{x}\left(i_{n+1}, \ldots, i_{n+k-1}\right) \\
& =\left[Z_{n}^{n+k}\left(x_{n}, x_{n+k}\right)\right]^{-1} \\
& \quad \times \exp \left[-U\left(x_{n}, i_{n+1}\right)-U\left(i_{n+k-1}, x_{n+k}\right)-\sum_{n+1 \leqslant r \leqslant n+k-2} U\left(i_{r}, i_{r+1}\right)\right]
\end{aligned}
$$

есть условная мера цилиндрического множества

$$
C\left(i_{n+1}, \ldots, i_{n+k-1}\right)=\left\{y \in X(G): y_{n+r}=i_{n+r}, 1 \leqslant r \leqslant k-1\right\}
$$

при условии $\sigma$-алгебры, порожденной случайными величинами $x_{r}, r \notin[n+1, n+k-1]$. 
4.10. ПРЕДЛОЖЕНИЕ. Мера $\mu \in \mathscr{M}(X(G), S)$ является гиббсовской мерой $c$ потенциалом $U$ в том и только том случае, когда для всех $n \in \mathbb{Z}$, всех иельх $k \geqslant 2$ и всех $i_{n}, \ldots, i_{n+k} \in V(G)$ таких, что $\mu\left(x_{n}=i_{n}, x_{n+k}=i_{n+k}\right)>0$, вицолняется равенство

$$
\begin{aligned}
& \mu\left(x_{n+1}=i_{n+1}, \ldots\right.\left., x_{n+k-1}=i_{n+k-1} \mid x_{n}=i_{n}, x_{n+k}=i_{n+k}\right) \\
&=\left[Z_{n}^{n+k}\left(i_{n}, i_{n+k}\right)\right]^{-1} \exp \left[-\sum_{r=0}^{k-1} U\left(i_{n+r}, i_{n+r+1}\right)\right] .
\end{aligned}
$$

Заметим, что правая часть (4.15) равна нулю, если последовательность $\gamma=$ $\left(i_{n}, \ldots, i_{n+k}\right)$ не является $G$-путем, и равна $Q(A, \gamma) / Q_{i_{n} i_{n+k}}(A, k)$, где $A=A_{f}$ (см. (4.1)), в противном случае.

ДокАЗАТЕльство. Если $\mu$-гиббсовская мера с потенщиалом $U$, то в силу (4.13), (4.14) условная мера $\mu_{x}\left(i_{1}, \ldots, i_{k}\right)$ измерима относительно $\sigma$-алгебры, порожденной лишь случайными величинами $x_{n}$ и $x_{n+k}$. В таком случае она совпадает с условной мерой того же цилиндра $C\left(i_{n+1}, \ldots, i_{n+k-1}\right)$ при условии упомянутой $\sigma$-алгебры, а для этой последней меры равенство (4.15) вьполняется по определению.

Обратно, пусть мера $\mu$ удовлетворяет сформулированному условию. Тогда (4.15) для нее вьполняется в обобшенном виде, а именно: для любых $n \in \mathbb{Z}$, любых целых $k \geqslant 2, l \geqslant 1$ и любых $i_{s} \in V(G), n-l \leqslant s \leqslant n+k+l$, таких, что

$$
\mu\left(x_{n-l}=i_{n-l}, \ldots, x_{n}=i_{n}, x_{n+k}=i_{n+k}, \ldots, x_{n+k+l}=i_{n+k+l}\right)>0
$$

имеет место равенство

$$
\mu\left(C \mid C^{-} \cap C^{+}\right)=\left[Z_{n}^{n+k}\left(i_{n}, i_{n+k}\right)\right]^{-1} \exp \left[-\sum_{r=0}^{k-1} U\left(i_{n+r}, i_{n+r+1}\right)\right]
$$

где

$$
\begin{aligned}
C & =\left\{x \in X(G): x_{n+1}=i_{n+1}, \ldots, x_{n+k-1}=i_{n+k-1}\right\}, \\
C^{-} & =\left\{x \in X(G): x_{n-l}=i_{n-l}, \ldots, x_{n}=i_{n}\right\}, \\
C^{+} & =\left\{x \in X(G): x_{n+k}=i_{n+k}, \ldots, x_{n+k+l}=i_{n+k+l}\right\} .
\end{aligned}
$$

В самом деле, представив $C^{-}$и $C^{+}$соответственно в виде $C^{-}=C_{2}^{-} \cap C_{1}^{-}, C^{+}=C_{1}^{+} \cap$ $\mathrm{C}_{2}^{+}$, где

$$
\begin{aligned}
& C_{2}^{-}=\left\{x \in X(G): x_{n-l}=i_{n-l}\right\}, \\
& C_{1}^{-}=\left\{x \in X(G): x_{n-l+1}=i_{n-l+1}, \ldots, x_{n}=i_{n}\right\}, \\
& C_{1}^{+}=\left\{x \in X(G): x_{n+k}=i_{n+k}, \ldots, x_{n+k+l-1}=i_{n+k+l-1}\right\}, \\
& C_{2}^{+}=\left\{x \in X(G): x_{n+k+l}=i_{n+k+l}\right\},
\end{aligned}
$$


получим

$$
\begin{aligned}
\mu\left(C \mid C^{-} \cap C^{+}\right) & =\mu\left(C \mid C_{2}^{-} \cap C_{1}^{-} \cap C_{1}^{+} \cap C_{2}^{+}\right) \\
& =\frac{\mu\left(C_{1}^{-} \cap C \cap C_{1}^{+} \mid C_{2}^{-} \cap C_{2}^{+}\right)}{\mu\left(C_{1}^{-} \cap C_{1}^{+} \mid C_{2}^{-} \cap C_{2}^{+}\right)}
\end{aligned}
$$

(по предположению все события, выступающие в качестве условий, имеют положительную вероятность). Далее,

$$
\mu\left(C_{1}^{-} \cap C_{1}^{+} \mid C_{2}^{-} \cap C_{2}^{+}\right)=\sum_{C^{\prime}} \mu\left(C_{1}^{-} \cap C^{\prime} \cap C_{1}^{+} \mid C_{2}^{-} \cap C_{2}^{+}\right),
$$

где суммирование ведется по всем множествам $C^{\prime}$ вида

$C^{\prime}=\left\{x^{\prime} \in X(G): x_{n+1}^{\prime}=i_{n+1}^{\prime}, \ldots, x_{n+k-1}^{\prime}=i_{n+k-1}^{\prime}\right\}, \quad i_{n+1}^{\prime}, \ldots, i_{n+k-1}^{\prime} \in V(G)$.

Применим формулу (4.15) к числителю дроби в правой части (4.17) и к каждому слагаемому в правой части (4.18). С учетом равенства статистических сумм в числителе и знаменателе получим

$$
\mu\left(C \mid C^{-} \cap C^{+}\right)=\exp (-T)\left[\sum_{i_{n+1}^{\prime}, \ldots, i_{n+k-1}^{\prime} \in V(G)} \exp \left(-T_{1}-T_{2}-T_{3}-T_{4}-T_{5}\right)\right]^{-1}
$$

где

$$
\begin{aligned}
T & =\sum_{r=n-l}^{n+k+l-1} U\left(i_{r}, i_{r+1}\right), & T_{1} & =\sum_{r=n-l}^{n-1} U\left(i_{r}, i_{r+1}\right), \\
T_{2} & =U\left(i_{n}, i_{n+1}^{\prime}\right), & T_{3} & =\sum_{r=n+1}^{n+k-2} U\left(i_{r}^{\prime}, i_{r+1}^{\prime}\right), \\
T_{4} & =U\left(i_{n+k-1}^{\prime}, i_{n+k}\right), & T_{5} & =\sum_{r=n+k}^{n+k+l-1} U\left(i_{r}, i_{r+1}\right) .
\end{aligned}
$$

После сокращения общих множителей (4.19) примет вид

$$
\begin{aligned}
\mu\left(C \mid C^{-} \cap C^{+}\right) & =\frac{\exp \left[-\sum_{r=n}^{n+k-1} U\left(i_{r}, i_{r+1}\right)\right]}{\sum_{i_{n+1}^{\prime}, \ldots, i_{n+k-1}^{\prime} \in V(G)} \exp \left(-T_{2}-T_{3}-T_{4}\right)} \\
& =\frac{1}{Z_{n}^{n+k}\left(i_{n}, i_{n+k}\right)} \exp \left[-\sum_{r=n}^{n+k-1} U\left(i_{r}, i_{r+1}\right)\right] .
\end{aligned}
$$

Тем самым равенство (4.16) доказано. Из него видно, что $\mu_{x}\left(i_{n+1}, \ldots, i_{n+k}\right)$ (см. (4.14)) есть условная мера цилиндра $C\left(i_{n+1}, \ldots, i_{n+k-1}\right)$ при условии $\sigma$-алгебры, порожденной случайными величинами $x_{r}, r \in[n-l, n] \cup[n+k, n+k+l]$. Остается перейти к пределу при $l \rightarrow \infty$, что можно сделать в силу теоремы Леви о сходимости 
условных математических ожиданий относительно неубьвающей последовательности $\sigma$-алгебр (см., например, [31]). Предложение доказано.

Следуюшая теорема устанавливает тождественность множеств $f$-равновесных мер и $S$-инвариантных гиббсовских мер с потенциалом $U$, связанньм с $f$ формулой (4.1), и тем самьм - возможность определения последних как точек максимума функционала $P(X(G), f, \cdot)$. Этот вариационный принцип, восходящий к Гиббсу, впервые был сфформулирован и доказан для систем на бесконечных решетках любой размерности О. Лэнфордом и Д. Рюэлем [32] в предположении, что пространство значений спиновой переменной конечно (одно из двух утверждений, на которые естественно разбивается вариационный принцип, было ранее доказано Р. Л. Добрушиным [33]).

4.11. Теорема [25, теорема D]. Пусть $G$ - связный ориентированный граф, $f \in \mathscr{F}_{0}^{1}(G)$ и $A=A_{f}-$ матрица Больцмана, отвечающая функции $f$. Тогда:

1) если $R(A)>0$ и существует $f$-равновесная мера $\mu^{f} \in \mathscr{E}(X(G), S)$, то $\mu^{f}-$ гиббсовская мера с потенциалом $U$, где $U$ u $f$ связань соотношениe.M (4.1);

2) если существует гиббсовская мера $\mu \in \mathscr{M}(X(G), S)$ с потенциалом $U$, то $R(A)>0, \mu \in \mathscr{E}(X(G), S)$ и $\mu-f$-равновесная мера, т.е. $\mu=\mu^{f}$.

4.12. ЗАмЕЧАния. 1. Сопоставление теорем 4.6 и 4.11 приводит к необходимому и достаточному условию существования $S$-инвариантной гиббсовской меры с потенциалом $U$, которое состоит в положительной возвратности матрицы Больцмана $A_{f}$ (в предположении, что вьполняется (4.1)). Для частного случая, когда $A_{f}$ не содержит нулевых элементов, т.е. $U$ принимает лиш конечные значения, последний результат был другим методом получен в работе Кестена [34], где найдены также условия существования гиббсовских мер, не являющихся $S$-инвариантными.

2. Вполне может случиться, что для гиббсовской меры $\mu$ с потенциалом $U$, связанным с функцией $f \in \mathscr{F}_{0}^{1}(G)$ соотношением (4.1), вьполняются равенства

$$
h_{\mu}(S)=\infty, \quad \int_{X(G)} f d \mu=-\infty
$$

(см. [25]). Именно по этой причине, определяя функционал $P(X(G), f, \cdot)$ в случае, когда $\inf _{x} f(x)=-\infty$, нельзя ограничиться мерами $\mu$ с $h_{\mu}(S)<\infty$ и необходима регуляризация. Только после нее удается доказать, что всякая гиббсовская мера является равновесной.

В завершение этого параграф̆а рассмотрим вопрос о связи между $f$-равновесными и $f_{n}$-равновесными мерами, где $f_{n}=\Phi_{n} f$ - функция, в которую переходит $f$ под действием $n$-редукции $\Phi_{n}, n \geqslant 2($ см. $\S \S 1,2)$. Функции $f$ и $f_{n}$ определены соответственно на пространствах $X(G)$ и $X\left(G_{n}\right)$, где $G_{n}=\Phi_{n} G$. При этом по условию $f \in \mathscr{F}_{0}^{1}(G)$, откуда следует, что $f_{n} \in \mathscr{F}_{0}^{0}\left(G_{n}\right)$. С помощью $\Phi_{n}$ любую борелевскую меру $\mu$, определенную на $X(G)$, можно перенести на $X\left(G_{n}\right)$, положив

$$
\left(\Phi_{n} \mu\right)(B)=\mu\left(\Phi_{n}^{-1} B\right), \quad B \in \mathscr{B}\left(X\left(G_{n}\right)\right) .
$$

4.13. ПРЕДЛОЖЕНИЕ. При всех $n \geqslant 2$ справедливо равенство $\mu_{\Phi_{n} f}=\Phi_{n} \mu^{f}$. 
ДокАЗАТЕльство. Прежде всего заметим, что $f$-равновесная и $\Phi_{n} f$-равновесная меры могут сушествовать лиш одновременно. Это непосредственно вытекает из теоремы 4.6 и предложения 2.12 .

Пусть, как и раньше, $f(x)=-U\left(x_{0}, x_{1}\right), x=\left(x_{i}, i \in \mathbb{Z}\right) \in X(G)$. Тогда $f_{n}=\Phi_{n} f$ можно представить в виде $f_{n}(y)=-U_{n}\left(y_{0}\right), y=\left(y_{i}, i \in \mathbb{Z}\right) \in X\left(G_{n}\right)$. Из определения $\Phi_{n}$ видно, что $U$ и $U_{n}$ связаны следующим образом: если $v=\left(i_{1}, \ldots, i_{n}\right) \in V\left(G_{n}\right)$, где $i_{1}, \ldots, i_{n} \in V(G)$, то $U_{n}(v)=U\left(i_{1}, i_{2}\right)$. С помошью предложения 4.10 теперь нетрудно проверить, что если $\mu$-гиббсовская мера на $X(G)$ с потенциалом $U$, то $\Phi_{n} \mu$ - гиббсовская мера на $X\left(G_{n}\right)$ с потенциалом $U_{n}$ (при этом достаточно ограничиться случаем $n=2$ ), после чего остается применить теорему 4.11 , утверждающую, что в рассматриваемом случае гиббсовская мера и равновесная мера - это одно и то же.

Глава 2.

Два класса инвариантных мер, связанных с СЦМ. Метод субдифференциалов

Основная цель этой главы - выяснить асимптотическое поведение двух классов вероятностных мер, естественным образом порождаемых бесконечной СЦМ $(X(G), S)$ и заданной на $X(G)$ непрерьвной функцией $f$. Мы будем пользоваться методом субдифференциалов (касательных функционалов - по терминологии Рюэля [5]).

\section{$\S$ 5. Субдифференциал топологического давления}

Вместе с пространством $X(G)$ удобно рассматривать его пополнение относительно метрики $d_{2}$ (см. $\left.\S 1\right)$, которое будем обозначать $\bar{X}(G)$. Пространство $\bar{X}(G)$, как нетрудно понять, допускает следующее описание. Отождествив $V(G)$ с множеством натуральных чисел $\mathbb{N}$, присоединим к нему точку $\infty$ и продолжим $\rho_{2}$ на множество $\overline{\mathbb{N}}=\mathbb{N} \cup\{\infty\}$ по формуле $\bar{\rho}_{2}(i, j)=\left|i^{-1}-j^{-1}\right|, i, j \in \overline{\mathbb{N}}$ (здесь, как обьчно, $\infty^{-1}=0$ ). Если теперь задать на $\bar{X}=\overline{\mathbb{N}}^{\mathbb{Z}}$ метрику $\bar{d}_{2}$ с помошью равенства $(1.1)$ при $\theta=1 / 2$, $\rho=\bar{\rho}_{2}$ и взять замыкание множества $X(G)$ в $\bar{X}$, то получится $\bar{X}(G)$. Для нас важно, что $\bar{X}(G)$ (как и все $\bar{X}$ ) оказывается не только полньм, но и компактным пространством и что на него можно продолжить преобразование $S$. В зависимости от структуры графа $G$ пространство $\bar{X}(G)$ содержит или не содержит последовательность $x^{\infty}=\left(x_{n}, n \in \mathbb{Z}\right)$, в которой $x_{n}=\infty$ при всех $n \in \mathbb{Z}$. Сейчас мы свяжем это свойство со свойствами матрищ $A_{f}$, отвечаюших функциям $f \in \mathscr{F}_{0}^{1}(G)$.

5.1. ПРЕДЛОЖЕНИЕ. 1) Если матрица $A_{f_{0}}$, әде $f_{0} \equiv 0$, допустима, то $x^{\infty} \in$ $\bar{X}(G)$

2) если существует функция $f \in \mathscr{F}_{0}^{1}(G)$, для которой $R\left(A_{f}\right)>0$ и матрица $A_{f}$ не является устойчиво-полохсительной, то $x^{\infty} \in \bar{X}(G)$.

ДокАЗАТЕЛЬСТВо. 1) Предположим, что $x^{\infty} \notin \bar{X}(G)$. Из определения метрики $d_{2}$ следует, что тогда для некоторых $n_{0} \in \mathbb{N}$ и $V_{0} \subset V(G)$, где $\# V_{0}<\infty$, всякий $G$-путь длины $\geqslant n_{0}$ содержит хотя бы одну вершину из $V_{0}$. Зафиксировав эти $n_{0}$ и $V_{0}$, выберем для каждой вершины $v \in V(G) \backslash V_{0}$ кратчайший путь $\gamma^{-}$, ведуший из $V_{0}$ в $v$, и кратчайший путь $\gamma^{+}$, ведуший из $v$ в $V_{0}$. Ясно, что лишш начальная и конечная вершины пути $\gamma(v)=\gamma^{-} \gamma^{+}$принадлежат $V_{0}$. Поскольку $\# V(G)=\infty$, сушествует такое множество $V_{1} \subset V(G)$, что \# $V_{1}=\infty$ и $\gamma\left(v^{\prime}\right) \neq \gamma\left(v^{\prime \prime}\right)$ при $v^{\prime}, v^{\prime \prime} \in V_{1}, v^{\prime} \neq v^{\prime \prime}$. 
В силу выбора $n_{0}$ и $V_{0}$ лиш конечное число путей $\gamma(v), v \in V_{1}$, имеет длину $\geqslant n_{0}$. Поэтому найдутся такие $v_{1}, v_{2} \in V_{0}, n<n_{0}$ и $V_{2} \subset V_{1}$, что $\# V_{2}=\infty$ и при всех $v \in V_{2}$ путь $\gamma(v)$ ведет из $v_{1}$ в $v_{2}$. Но вследствие допустимости матрицы $A_{f_{0}}$ число $G$-путей, ведуших из $v_{1}$ в $v_{2}$, конечно. Полученное противоречие доказьвает, что $x^{\infty} \in \bar{X}(G)$.

2) Возьмем произвольную исчерпьвающую последовательность конечных главных подграфов $G_{n} \subset G$ (см. определение 3.9 ) и произвольную возрастаюшую последовательность натуральных чисел $l_{n}$. Предположим, что для любого $n$ найдется $G$-путь $\gamma_{n}$ длины $l\left(\gamma_{n}\right) \leqslant l_{n}$, все вершины которого принадлежат множеству $V(G) \backslash V\left(G_{n}\right)$. Произвольньм образом дополнив $\gamma$ до двусторонне-бесконечного пути $x^{n} \in X(G) \subset$ $\bar{X}(G)$, нетрудно убедиться в том, что при подходящем выборе чисел $m_{n} \in \mathbb{Z}$ имеет место сходимость (в $\bar{X}) S^{m_{n}} x^{n} \rightarrow x^{\infty}(n \rightarrow \infty)$; тем самым, $x^{\infty} \in \bar{X}(G)$. Поэтому в случае, когда $x^{\infty} \notin \bar{X}(G)$, сушествуют конечньй связный подграф $G_{0} \subset G$ и число $l \in \mathbb{N}$ такие, что длина любого $G$-пути $\gamma$, не проходящего через $V\left(G_{0}\right)$, меньше $l$. Положим $\psi(z)=\sum_{\gamma \in \Gamma_{0}} Q\left(A_{f}, \gamma\right) z^{l(\gamma)}$, где $\Gamma_{0}$ - множество $G$-путей, которые начинаются и кончаются в $V\left(G_{0}\right)$, но не проходят через $V\left(G_{0}\right)$. Из сказанного следует, что $\psi(z)-$ многочлен, и теорема 3.8 позволяет заключить, что $A_{f}$ - устойчиво-положительная матрица. Предложение доказано.

В дальнейшем мы постоянно будем иметь дело с определенными на $\bar{X}(G)$ борелевскими зарядами, в частности, с вероятностными мерами, сосредоточенными на $X(G)$. Говоря о сходимости последовательностей $\left\{\mu_{n}\right\}$ таких зарядов, мы будем, если не оговорено противное, иметь в виду их слабую сходимость (часто назьваемую также $*$-слабой), т.е. сходимость последовательности $\int_{\bar{X}(G)} f d \mu_{n}$ для всех $f \in C(\bar{X}(G))$. Заметим, однако, что если предел последовательности $\left\{\mu_{n}\right\}, \mu_{n} \in \mathscr{M}(X(G))$, также сосредоточен на $X(G)$, то в силу обших теорем теории меры (см., например, [31, гл. 3 , $\S 1$, замечание 1]) в качестве $f$ можно брать любые функции из $C(X(G))$, т.е. имеет место и слабая сходимость мер на $X(G)$. Некоторые утверждения $\S \S 6$ и 7 относятся именно к такой ситуации.

В $\S 4$ для любой функции $f \in \mathscr{F}_{0}^{1}(G)$, которой отвечает матрица Больцмана $A_{f}$ с $R\left(A_{f}\right)>0$, и любой меры $\mu \in \mathscr{E}(X(G), S)$ была определена величина $P(X(G), f, \mu)$ - давление в состоянии $\mu$, точная верхняя грань которой равна топологическому давлению $P^{\operatorname{int}}(X(G), S, f)$ (см. теорему 4.4). Зафиксировав произвольную функцию $f \in$ $\mathscr{F}_{0}^{1}(G)$, положим

$$
P_{f}(g)=P^{\mathrm{int}}(X(G), S, f+g), \quad g \in C(\bar{X}(G)) .
$$

5.2. Лемма. При $P^{\operatorname{int}}(X(G), S, f)<\infty$ функиионал $P_{f}$ обладает следующими свойствами:

1) если $g, g_{n} \in C(\bar{X}(G)), n \in \mathbb{N}, u \lim _{n \rightarrow \infty}\left\|g_{n}-g\right\|=0$, mo $\lim _{n \rightarrow \infty}\left|P_{f}\left(g_{n}\right)-P_{f}(g)\right|$ $=0$ (непрерьвность);

2) $P_{f}\left(S^{*} g\right)=P_{f}(g)$, әде $\left(S^{*} g\right)(x)=g(S x), x \in \bar{X}(G)(S$-инвариантность);

3) если $g_{1}, g_{2} \in C(\bar{X}(G))$ и $g_{1}(x) \leqslant g_{2}(x)$ при всех $x \in \bar{X}(G)$, то $P_{f}\left(g_{1}\right) \leqslant$ $P_{f}\left(g_{2}\right)$ (монотонность);

4) если $g_{1}, g_{2} \in C(\bar{X}(G))$ u $g=p_{1} g_{1}+p_{2} g_{2}$, где $p_{1}, p_{2} \geqslant 0, p_{1}+p_{2}=1$, то $P_{f}(g) \geqslant p_{1} P_{f}\left(g_{1}\right)+p_{2} P_{f}\left(g_{2}\right)$ (вьликллость); 
5) если $\mathscr{E}_{f}(X(G), S)$ - совокупность мер $\mu \in \mathscr{E}(X(G), S)$, для которых $f \in$ $L_{1}(\mu)$, mo

$$
P_{f}(g)=\sup _{\mu \in \mathscr{E}_{f}(X(G))}\left[P(X(G), f, \mu)+\int_{X(G)} g d \mu\right], \quad g \in C(\bar{X}(G))
$$

(вариационный принцип).

ДокАЗАТельство. Непрерьвность $P_{f}$ следует из непрерьвности по $g$ функции $P^{\operatorname{int}}\left(X\left(G_{0}\right), S, f+g\right)$, где $G_{0}$ - произвольньй конечный подграф графа $G$, и вариационного принципа для $P^{\text {int }}\left(X\left(G_{0}\right), S, f+g\right)$. Точно так же доказывается $S$-инвариантность. Вьпуклость и монотонность $P_{f}$ вытекают из соотношения (5.2). Приступим к его доказательству.

Для краткости обозначим правую часть (5.2) через $P_{f}^{1}(g)$. Очевидно,

$$
P_{f}^{1}(g) \geqslant \sup _{G_{0} \in \mathcal{G}_{0}(G)} \sup _{\mu \in \mathscr{E}\left(X\left(G_{0}\right), S\right)}\left[P(X(G), f, \mu)+\int_{X\left(G_{0}\right)} g d \mu\right], \quad g \in C(\bar{X}(G)),
$$

где, как и в $\S 1, \mathscr{G}_{0}(G)$ - совокупность конечных подграфов графа $G$. Если $G_{0} \in \mathscr{G}_{0}(G)$, то $\left.f\right|_{X\left(G_{0}\right)}$ - ограниченная функция и при $\mu \in \mathscr{E}\left(X\left(G_{0}\right), S\right)$ в силу предложения 4.3 (см. также (4.4) и теорему 4.4)

$$
P(X(G), f, \mu)=h_{\mu}(S)+\int_{X\left(G_{0}\right)} f d \mu .
$$

Поэтому при $G_{0} \in \mathscr{G}_{0}(G)$

$$
\begin{aligned}
\sup _{\mu \in \mathscr{E}\left(X\left(G_{0}\right), S\right)}\left[P(X(G), f, \mu)+\int_{X\left(G_{0}\right)} g d \mu\right] \\
=\sup _{\mu \in \mathscr{E}\left(X\left(G_{0}\right), S\right)}\left[h_{\mu}(S)+\int_{X\left(G_{0}\right)}(f+g) d \mu\right] \\
=\sup _{\mu \in \mathscr{M}\left(X\left(G_{0}\right), S\right)}\left[h_{\mu}(S)+\int_{X\left(G_{0}\right)}(f+g) d \mu\right]=P\left(X\left(G_{0}\right), S, f+g\right)
\end{aligned}
$$

(по поводу предпоследнего равенства см. замечание 4.5 , последнее вытекает из вариационного принципа (1.5)). Отсюда с учетом (5.3) и (1.15) получаем

$$
P_{f}^{1}(g) \geqslant P^{\mathrm{int}}(X(G), S, f+g)=P_{f}(g) .
$$

Чтобы доказать обратное неравенство, вначале заметим, что достаточно сделать это для функций $g$, ограничения которых на $X(G)$ являются локальными функциями (см. определение 1.10), поскольку такие функции всюду плотны в $C(\bar{X}(G))$, а $P_{f}(g)$ и $P_{f}^{1}(g)$ непрерьвны по $g$. Из $S$-инвариантности $P_{f}(g)$ и $P_{f}^{1}(g)$ (инвариантность $P_{f}^{1}(g)$ очевидна) вытекает, что при этом можно ограничиться функциями $g$, для которых $\left.g\right|_{X(G)} \in \bigcup_{k=0}^{\infty} \mathscr{F}_{0}^{k}(G)$. 
Пусть $g$ - одна из таких функщий и $g^{\prime}=\left.g\right|_{X(G)}$. Не ограничивая обшности, можно считать, что $g^{\prime} \in \mathscr{F}_{0}^{k}(G)$ при некотором $k>1$. Отображение $\Phi_{k}$ переводит граф $G$ в граф $G_{k}$, пространство $X(G)$ - в пространство $X\left(G_{k}\right)$, а функции $f$ и $g^{\prime}-$ в $f_{k}$ и $g_{k}^{\prime}$.

Зафиксируем теперь произвольную меру $\mu \in \mathscr{E}_{f}(X(G), S)$ и положим $\mu_{k}=\Phi_{k} \mu$. Применив предложение 4.3 сначала к $\mu$ и $f$, а затем к $\mu_{k}$ и $f_{k}$ и воспользовавшись тем, что $f_{k}, g_{k}^{\prime} \in \mathscr{F}_{0}^{1}\left(G_{k}\right)$, получим

$$
\begin{aligned}
P(X(G), f, \mu)+\int_{X(G)} g d \mu & =h_{\mu}(S)+\int_{X(G)} f d \mu+\int_{X(G)} g d \mu \\
& =h_{\mu_{k}}(S)+\int_{X\left(G_{k}\right)}\left(f_{k}+g_{k}^{\prime}\right) d \mu_{k} \\
& =P\left(X\left(G_{k}\right), f_{k}+g_{k}^{\prime}, \mu_{k}\right) .
\end{aligned}
$$

В силу теоремы 4.4

$$
P\left(X\left(G_{k}\right), f_{k}+g_{k}^{\prime}, \mu_{k}\right) \leqslant P^{\mathrm{int}}\left(X\left(G_{k}\right), S, f_{k}+g_{k}^{\prime}\right),
$$

а так как $\Phi_{k}$ - гомеоморфизм, справедливо равенство

$$
P^{\mathrm{int}}\left(X\left(G_{k}\right), S, f_{k}+g_{k}^{\prime}\right)=P^{\mathrm{int}}\left(X(G), S, f+g^{\prime}\right) .
$$

Таким образом,

$$
P(X(G), f, \mu)+\int_{X(G)} g d \mu \leqslant P_{f}(g), \quad \mu \in \mathscr{E}_{f}(X(G), S)
$$

что вместе с (5.4) приводит к (5.2). Лемма доказана.

5.3. ОПРЕДЕЛЕниЕ [47]. Пусть $F$ - непрерьвная вьпуклая функция, заданная на банаховом пространстве $H$. Линейный функционал $L \in H^{*}$ называется субградиентом функиии $F$ в точке $h_{0} \in H$, если при всех $h \in H$ выполняется неравенство

$$
L(h) \leqslant F\left(h_{0}+h\right)-F\left(h_{0}\right) .
$$

Множество всех таких $L$ называется субдифференциалом и обозначается символом $\partial F\left(h_{0}\right)$. Если (5.6) имеет место при всех $h \in H$ с $\|h\| \leqslant \delta$, то $L$ называется $\delta$-субградиентом. Множество всех $\delta$-субградиентов назовем $\delta$-субдиффференциалом.

Цель этого параграфа - найти $\partial P_{f}(0)$. Но начнем мы с простой леммы, показывающей, что понятия $\delta$-субградиента и $\delta$-субдифференциала являются, по существу, лишними. Хотя эта лемма будет использоваться лишь в $\S 7$, ее естественно сформулировать и доказать здесь.

5.4. ЛЕммА. При $\delta>0$ всякий $\delta$-субградиент является градиентом. 
ДокАЗАТЕльство. Пусть линейный функционал $L$ служит $\delta$-субградиентом непрерьвной вьпуклой функции $F: H \rightarrow \mathbb{R}$ в точке $x \in H$. Для любого $y \in H$ подберем такое $\varepsilon=\varepsilon_{y}>0$, что $\|\varepsilon y\|<\delta$. По определению $\delta$-субградиента и в силу линейности функционала $L$

$$
F(x+\varepsilon y) \geqslant \varepsilon L(y)+F(x),
$$

а вследствие выпуклости $F$

$$
F(x+\varepsilon y) \leqslant(1-\varepsilon y) F(x)+\varepsilon F(x+y) .
$$

Из этих двух неравенств после очевидных преобразований получаем

$$
F(x+y) \geqslant F(x)+L(y)
$$

что и требовалось доказать.

Введем производные по направлениям. Пусть

$$
\begin{array}{ll}
\omega_{f}^{+}(g)=\lim _{t \rightarrow+0} \frac{1}{t}\left[P_{f}(t g)-P_{f}(0)\right], & g \in C(\bar{X}(G)), \\
\omega_{f}^{-}(g)=\lim _{t \rightarrow-0} \frac{1}{t}\left[P_{f}(t g)-P_{f}(0)\right], & g \in C(\bar{X}(G))
\end{array}
$$

(вследствие выпуклости $P_{f}$ выражения, стоящие под знаком предела, не убьвают по $t$ ). Если $\omega_{f}^{-}(g)=\omega_{f}^{+}(g)$, то функция $t \mapsto P_{f}(t g)$ дифференцируема в точке $t=0$. Из монотонности $P_{f}$ вытекает, что если $g \geqslant 0$, то $\omega_{f}^{-}(g), \omega_{f}^{+}(g) \geqslant 0$.

\section{5. ОПРЕДЕЛЕНИЕ. Назовем множество}

$$
C=\left\{x \in X(G): x_{n}=v_{n}, n \in Z\right\}, \quad v_{n} \in V(G),
$$

где $Z \subset \mathbb{Z}$ и \# $Z<\infty$, иилиндрическим множеством (иилиндром) в $X(G)$ с носиmелем $\operatorname{supp}(C)=Z$. Цилиндры с носителем вида $\{k, \ldots, k+l\}, k, l \in \mathbb{Z}, l \geqslant 0$, будем называть сплошнылми. Если для такого цилиндра $v_{k}=v_{k+l}=v \in V(G)$, будем называть его $v$-иилиндром.

Всякий сплошной цилиндр имеет вид

$$
C(k, \gamma)=\left\{x \in X(G):\left(x_{k}, \ldots, x_{k+l}\right)=\gamma\right\}, \quad k, l \in \mathbb{Z}, \quad l>0,
$$

где $\gamma$ - некоторьй $G$-путь, если $l>0$, и $\gamma \in V(G)$, если $l=0$. Всякий цилиндр с одноточечным носителем является, очевидно, $v$-цилиндром при некотором $v \in V(G)$, а все остальные $v$-цилиндры имеют вид (5.10) с $\gamma \in \Gamma_{v v}(G, l), l \geqslant 1$. Ниже мы будем, как правило, писать $C(v)$ вместо $C(0, v)$ и $C(\gamma)$ вместо $C(0, \gamma)$.

Заменив в определении $5.5 X(G)$ на $\bar{X}(G)$, можно точно так же определить цилиндры в $\bar{X}(G)$.

Важно отметить два обстоятельства: во-первых, для любого цилиндра $C \subset X(G)$ его замькание $\bar{C} \subset \bar{X}(G)$ является не только замкнутым, но и открытым множеством, вследствие чего характеристическая функция $\mathbf{1}_{\bar{C}}$ непрерьвна (легко проверить, что $\bar{C}$ определяется тем же равенством (5.9), в котором на этот раз $x \in \bar{X}(G))$; во-вторых, $\bar{C}$ есть цилиндр в $\bar{X}(G)$ и $\bar{C} \cap X(G)=C$, откуда вытекает, что ограничение функции $\mathbf{1}_{\bar{C}}$ на $X(G)$ совпадает с $\mathbf{1}_{C}$. 
5.6. Лемма. Пусть $A_{f}$ - матрица Больимана, отвечающая функиии $f \in$ $\mathscr{F}_{0}^{1}(G)$, и $g=\mathbf{1}_{\bar{C}}$, где $C \subset X(G)$ - произвольньй непустой сплошной чилиндр. Предположим, что $R\left(A_{f}\right)>0$. Тогда:

1) если матрица $A_{f}$ невозвратна или нуль-возвратна, то $\omega_{f}^{-}(g)=\omega_{f}^{+}(g)$ $=0$

2) если матрица $A_{f}$ устойчиво-положсительна, то $\omega_{f}^{-}(g)=\omega_{f}^{+}(g)=\mu^{f}(C)$, где $\mu^{f}$ - равновесная мера, отвечающая функции $f$;

3) если матрица $A_{f}$ неустойчиво-положительна, то $\omega_{f}^{-}(g)=0, \omega_{f}^{+}(g)=$ $\mu^{f}(C)$.

ДокАЗАТЕЛЬСтво. В силу п. 2 леммы 5.2 можно считать, что $\operatorname{supp}(C)=\{0, \ldots, l\}$, $l \geqslant 0$. Введем функции $f_{l+1}=\Phi_{l+1} f, g_{l+1}^{\prime}=\Phi_{l+1} g^{\prime}$, где $g^{\prime}=\left.g\right|_{X(G)}$, и воспользуемся равенством

$$
P^{\text {int }}\left(X(G), S, f+t g^{\prime}\right)=P^{\text {int }}\left(X\left(G_{l+1}\right), S, f_{l+1}+t g_{l+1}^{\prime}\right), \quad t \in \mathbb{R}
$$

(cp. (5.5)). По предположению $R\left(A_{f}\right)>0$. Из (5.2) и теоремы 4.4 следует, что тогда

$$
P^{\text {int }}\left(X(G), S, f+t g^{\prime}\right) \leqslant P^{\text {int }}(X(G), S, f)+|t| M(g)=-\ln R\left(A_{f}\right)+|t|<\infty,
$$

где $M(g)=\max _{x \in \bar{X}(G)}|g(x)|=1$. Значит (см. (5.7)), $P^{\text {int }}\left(X\left(G_{l+1}\right), S, f_{l+1}+t g_{l+1}^{\prime}\right)$ $<\infty$ и по той же теореме 4.4

$$
P^{\mathrm{int}}\left(X\left(G_{l+1}\right), S, f_{l+1}+t g_{l+1}^{\prime}\right)=-\ln R\left(A^{t}\right),
$$

где $A^{t}$ - матрица Больцмана, отвечающая функции $f_{l+1}+t g_{l+1}^{\prime}$ (по построению $\left.f_{l+1}, t g_{l+1}^{\prime} \in \mathscr{F}_{0}^{0}\left(G_{l+1}\right)\right)$.

Как мы предположили вьше, $\operatorname{supp}(C)=\{0, \ldots, l\}$. В последуюших рассуждениях ограничимся случаем $l \geqslant 1$ (при $l=0$ все гораздо проше). Тогда $C=C(0, \gamma)$, где $\gamma$ - некоторьй $G$-путь, одновременно служаший вершиной графра $G_{l+1}$. Обозначим эту вершину через $v$. Из определения матрищы $A_{l+1}^{t}$ следует, что

$$
\varphi_{v v}^{0}\left(A_{l+1}^{t}, z\right)=e^{t} \varphi_{v v}^{0}\left(A_{l+1}^{t}, z\right), \quad|z|<R_{v v}^{0}\left(A_{l+1}\right),
$$

где $A_{l+1}=A_{l+1}^{0}$, и, тем самьм,

$$
R_{v v}^{0}\left(A_{l+1}^{t}\right)=R_{v v}^{0}\left(A_{l+1}\right), \quad t \in \mathbb{R}
$$

Если матрица $A$ невозвратна, нуль-возвратна или неустойчиво-положительна, то, согласно предложению 2.12 точно такими же свойствами обладает матрица $A_{l+1}$ и (см. лемму 2.6 и следствие 2.10 )

$$
R\left(A_{l+1}\right)=R_{v v}^{0}\left(A_{l+1}\right), \quad \varphi_{v v}^{0}\left(A_{l+1}, R\left(A_{l+1}\right)\right) \leqslant 1 .
$$

Но тогда в силу (5.13), (5.14) при $t<0$

$$
\varphi_{v v}^{0}\left(A_{l+1}^{t}, R_{v v}^{0}\left(A_{l+1}^{t}\right)\right)<1
$$


и, следовательно,

$$
R\left(A_{l+1}^{t}\right)=R_{v v}^{0}\left(A_{l+1}^{t}\right)=R_{v v}^{0}\left(A_{l+1}\right) .
$$

Отсюда с учетом (5.11), (5.12) заключаем, что во всех рассматриваемых случаях $\omega_{f}^{-}(g)=0$.

Если матрица $A$ (и, значит, $\left.A_{l+1}\right)$ невозвратна, то

$$
\varphi_{v v}\left(A_{l+1}, R\left(A_{l+1}\right)\right)<1
$$

и $(5.15),(5.16)$ выполняются при всех $t$, достаточно близких к нулю, а потому в этом случае $\omega_{f}^{+}(g)=0$. Во всех остальных случаях при $t>0$

$$
\begin{gathered}
\varphi_{v v}^{0}\left(A_{l+1}^{t}, R_{v v}^{0}\left(A_{l+1}^{t}\right)\right)>1, \quad R\left(A_{l+1}^{t}\right)<R_{v v}^{0}\left(A_{l+1}^{t}\right)=R_{v v}^{0}\left(A_{l+1}\right), \\
\varphi_{v v}^{0}\left(A_{l+1}, R\left(A_{l+1}^{t}\right)\right)=e^{-t}
\end{gathered}
$$

(последнее равенство вытекает из (5.13) и леммы 2.6).

Положим

$$
\varphi(r)=\varphi_{v v}^{0}\left(A_{l+1}, r\right), \quad 0 \leqslant r \leqslant R\left(A_{l+1}\right),
$$

и пусть $\psi:[0,1] \rightarrow\left[0, R\left(A_{l+1}\right)\right]-$ функция, обратная к $\varphi$. Функции $\varphi$ и $\psi$ монотонно возрастают и непрерьвно дифференцируемы (в концах отрезков производная заменяется соответствующей односторонней производной; при этом надо иметь в виду, что продифференцированньй ряд типа (2.6), определяюший производную $\varphi^{\prime}\left(R\left(A_{l+1}\right)\right)$, может расходиться, в этом случае непрерывность $\varphi^{\prime}$ в точке $R\left(A_{l+1}\right)$ понимается как стремление $\varphi^{\prime}(r)$ к бесконечности при $\left.r \rightarrow R\left(A_{l+1}\right)-0\right)$. Из $(5.7),(5.8),(5.17)$ с учетом сказанного получаем

$$
\begin{aligned}
\omega_{f}^{+}(g) & =\lim _{t \rightarrow+0} \frac{1}{t}\left[-\ln R\left(A_{l+1}^{t}\right)+\ln R\left(A_{l+1}\right)\right]=\lim _{t \rightarrow+0} \frac{1}{t}\left[-\ln \psi\left(e^{-t}\right)+\ln \psi(1)\right] \\
& =\lim _{t \rightarrow+0} \frac{1}{e^{-t}-1}\left[\ln \psi\left(e^{-t}\right)-\ln \psi(1)\right]=\lim _{s \rightarrow-0} \frac{1}{s}[\ln \psi(1+s)-\ln \psi(1)] \\
& =(\ln \psi)^{\prime}(1)=\frac{\psi^{\prime}(1)}{\psi(1)}=\frac{1}{R\left(A_{l+1}\right) \varphi^{\prime}\left(R\left(A_{l+1}\right)\right)} .
\end{aligned}
$$

Отсюда сразу следует (см. теорему 2.7, следствие 2.10 и предложение 2.12 ), что если матрица $A$ нуль-возвратна, то $\omega_{f}^{+}(g)=0$. Если же матрица $A$ положительно-возвратна, то в силу п. 2 замечания 4.8 правая часть $(5.19)$ равна $\mu^{f_{l+1}}\left(C_{l+1}(v)\right)$, где

$$
C_{l+1}(v)=\left\{x \in X\left(G_{l+1}\right): x_{0}=v\right\}
$$

(см. (4.12)), а в силу предложения 4.13

$$
\mu^{f_{l+1}}\left(C_{l+1}(v)\right)=\mu^{f}\left(\Phi_{l+1}^{-1} C_{l+1}(v)\right)=\mu^{f}(C) .
$$

Таким образом, в этом случае $\omega_{f}^{+}(g)=\mu^{f}(C)$.

Для завершения доказательства леммы осталось вычислить $\omega_{f}^{-}$в случае, когда матрица $A$ устойчиво-положительна. Это вычисление ничем не отличается от только что проведенного, так как теперь неравенства (5.17) и равенство (5.18) вьполняются не только при $t>0$, но и при всех достаточно близких к нулю $t<0$. Лемма доказана.

Переходя к изучению субдифференциалов $\partial P_{f}(g), g \in C(\bar{X}(G))$ (см. определение 5.3), вначале заметим, что множество $\mathscr{M}(\bar{X}(G), S)$ инвариантных относительно сдвига $S$ вероятностных борелевских мер на $\bar{X}(G)$ содержится в $C^{*}(\bar{X}(G))$. 
5.7. Лемма. Если $g \in C(\bar{X}(G)), \operatorname{mo} \partial P_{f}(g) \subset \mathscr{M}(\bar{X}(G), S)$.

ДоКАЗАТЕЛЬСтво. Пусть $g, \tilde{g} \in C(\bar{X}(G)), \tilde{g}(x) \geqslant 0$ при всех $x \in \bar{X}(G)$ и $\nu \in$ $\partial P_{f}(g)$. Пользуясь определением 5.3 и монотонностью $P_{f}$ (см. п. 3 леммы 5.2), получаем $\nu(\tilde{g}) \geqslant P_{f}(g)-P_{f}(g-\tilde{g}) \geqslant 0$, откуда видно, что $\nu$ - неотрицательный заряд. Пусть, далее, $g_{1}(x) \equiv 1$. В силу $(5.2)$

$$
P_{f}\left(g+g_{1}\right)-P_{f}(g)=1, \quad P_{f}\left(g-g_{1}\right)-P_{f}(g)=-1
$$

и, следовательно (см. определение 5.3), $\nu\left(g_{1}\right) \leqslant 1,-\nu\left(g_{1}\right) \geqslant-1$, т.е. $\nu\left(g_{1}\right)=1$. Это значит, что $\nu$ - вероятностная мера. Ее $S$-инвариантность вытекает из $S$-инвариантности $P_{f}$ (см. п. 2 леммы 5.2). Лемма доказана.

Прежде чем двигаться дальше, докажем еше одно вспомогательное утверждение, которое будет использоваться не только в этом, но и в других параграфах.

5.8. Лемма. Пусть граф $G$ связен и $\mu, \nu$-две $S$-инвариантные вероятностные меры на $\bar{X}(G)$, причем $\mu$ сосредоточена на $X(G)$, положительна на всех иилиндрах $C(v), v \in V(G)$, и эргодична. Тогда:

1) если для каждого $v \in V(G)$ найдется такое $\alpha_{v} \geqslant 0$, что для любого $v$-цилиндра $C \subset X(G)$ выполняется соотношение

$$
\nu(\bar{C})=\alpha_{v} \mu(C)
$$

то $\alpha_{v}$ не зависит от $v$ (можно положить $\left.\alpha_{v}=\alpha\right), \alpha \leqslant 1 u$

$$
\nu=\alpha \mu+(1-\alpha) \delta_{\infty},
$$

где $\delta_{\infty}$ - вероятностная мера, сосредоточенная в точке $x^{\infty}$ (для единообразия будем считать это равенство выполненныц даже при $x^{\infty} \notin$ $\bar{X}(G)$, если только $\alpha=1)$;

2) если для некоторого $v \in V(G)$ и любого v-цилиндра $C \subset X(G)$ выполняется соотношение $\nu(\bar{C})=\mu(C)$, то $\nu=\mu$.

ДокАЗАТЕльство. Доказательство пा. 1 и 2 начнем с общего для них рассуждения. Зафиксируем $v \in V(G)$ и произвольньй сплошной цилиндр $C=C(k, \gamma) \subset X(G)$ (см. (5.10)). Пусть $\gamma=\left(v_{0}, \ldots, v_{l}\right)$. Возьмем какие-нибудь пути $\gamma^{-}$и $\gamma^{+}$, ведушие из $v$ в $v_{0}$ и из $v_{l}$ в $v$ соответственно и не проходящие через $v$. Обозначим через $l^{-}, l^{+}$их длины. Ясно, что путь

$$
\gamma^{ \pm}= \begin{cases}\gamma^{-} \gamma \gamma^{+}, & l>0 \\ \gamma^{-} \gamma^{+}, & l=0\end{cases}
$$

является $v$-циклом. Ему отвечает $v$-цилиндр

$$
C\left(k-l^{-}, \gamma^{ \pm}\right)=\left\{x \in X(G):\left(x_{k-l^{-}}, \ldots, x_{k+l+l^{+}}\right)=\gamma^{ \pm}\right\}
$$


Пусть $\mathscr{C}(\gamma)$ - множество всех полученных таким образом $v$-цилиндров. Из построения видно, что все цилиндры, входящие в $\mathscr{C}(\gamma)$, являются подмножествами цилиндра $C$ и что любые два из них либо не пересекаются, либо совпадают; очевидно, в первом случае не пересекаются и замыкания любых двух таких цилиндров. По условию $\nu\left(\overline{C^{\prime}}\right)=\alpha_{v} \mu\left(C^{\prime}\right)$ для любого $C^{\prime} \in \mathscr{C}(\gamma)$. Следовательно,

$$
\nu(\bar{C}) \geqslant \alpha_{v} \sum_{C^{\prime} \in \mathscr{C}(\gamma)} \mu\left(C^{\prime}\right) .
$$

Вследствие эргодичности меры $\mu$ правая часть этого неравенства есть $\mu(C)$. Тем самым для любого сплошного цилиндра $C \subset X(G)$ выполняется неравенство

$$
\nu(\bar{C}) \geqslant \alpha_{v} \mu(C)
$$

В предположениях п. 2 это верно для фиксированного $v$, но зато $\alpha_{v}=1$, а в предположениях п. 1 - для любого $v \in V(G)$, откуда следует, что в последнем случае для любого сплошного цилиндра $C \subset X(G)$

$$
\nu(\bar{C}) \geqslant \alpha \mu(C)
$$

где $\alpha=\sup _{v \in V(G)} \alpha_{v}$. Сравнив (5.20) и (5.22) при $C=C(v)$, получим

$$
\alpha_{v} \mu(C(v)) \geqslant \alpha \mu(C(v))
$$

что с учетом положительности $\mu(C(v))$ приводит к неравенству $\alpha_{v} \geqslant \alpha$. Вследствие произвольности $v$ это означает, что $\alpha_{v} \equiv \alpha$. Если теперь подставить $C=C(w)$, $w \in V(G)$, в $(5.22)$ и просуммировать по всем $w$, получится неравенство

$$
\sum_{w \in V(G)} \nu(\bar{C}(w)) \geqslant \alpha \sum_{w \in V(G)} \mu(C(w)) .
$$

Так как множества $C(w)$ при разных $w$ не пересекаются, а $\mu$ и $\nu$-вероятностные меры, из (5.23) следует, что $\alpha \leqslant 1$. Таким образом, неравенства $(5.22),(5.23)$ с $\alpha \in[0,1]$ вьполняются в предположениях каждого из пп. 1,2 .

Ясно, что при $\alpha=1$ (5.23) может быть лиш равенством. Но тогда мера $\nu$ coсредоточена на $X(G)$ - это вытекает из ее $S$-инвариантности и очевидного соотношения

$$
\bar{X}(G) \backslash \bigcup_{w \in V(G)} \bar{C}(w)=C(\infty),
$$

где $C(\infty)=\left\{x \in \bar{X}(G): x_{0}=\infty\right\}$. В таком случае (5.22) можно переписать в виде $\nu(C) \geqslant \mu(C)$, где $C$ - произвольньй сплошной цилиндр. Поскольку $\mu$ и $\nu-$ вероятностные меры, это неравенство на самом деле является равенством. Из него видно, что $\mu$ и $\nu$ совпадают на всех сплошных цилиндрах, а тогда они совпадают на всех борелевских множествах в $X(G)$. Тем самьм утверждение п. 2 и соотношение (5.21) при $\alpha=1$ доказаны. 
При $\alpha<1$ определим на $\bar{X}(G)$ заряд $\tilde{\nu}=(1-\alpha)^{-1}(\nu-\alpha \mu)$. Согласно (5.22) он неотрицателен на всех цилиндрах в $\bar{X}(G)$, имеюших вид $\bar{C}$, где $C$ - сплошной цилиндр в $X(G)$. Но на всех остальных сплошных цилиндрах в $\bar{X}(G)$ мера $\mu$ равна нулю (как легко понять, такие цилиндры не пересекаются с $X(G))$. Значит, заряд $\tilde{\nu}$ неотрицателен на всех без исключения сплошных цилиндрах в $\bar{X}(G)$. Отсюда следует, что он неотрицателен на всех борелевских множествах и тем самым является $S$-инвариантной вероятностной борелевской мерой на $\bar{X}(G)$. В силу $(5.20),(5.24) \tilde{\nu}(C(\infty))=1$, а тогда и

$$
\tilde{\nu}\left(x^{\infty}\right)=\tilde{\nu}\left(\bigcap_{n \in \mathbb{Z}} S^{n} C(\infty)\right)=1,
$$

т.е. $\tilde{\nu}=\delta_{\infty}$, что приводит к соотношению (5.21) и в случае $\alpha<1$. Лемма доказана.

Теперь можно сформулировать основной результат этого параграфа.

5.9. Теорема. Пусть матрица Больимана $A_{f}$, где $f \in \mathscr{F}_{0}^{1}(G)$, удовлетворяет условию $R\left(A_{f}\right)>0$. Тогда

1) если матрица $A_{f}$ невозвратна или нуль-возвратна, то $\partial P_{f}(0)=\left\{\delta_{\infty}\right\}$;

2) если матрица $A_{f}$ устойчиво-положительна, то $\partial P_{f}(0)=\left\{\mu^{f}\right\}$;

3) если матрица $A_{f}$ неустойчиво-положительна, то

$$
\begin{aligned}
& \left\{c \mu^{f}+(1-c) \delta_{\infty}, 0 \leqslant c \leqslant 1\right\} \subset \partial P_{f}(0) \\
& \subset\left\{c \mu^{f}+(1-c) \bar{\mu}, 0 \leqslant c \leqslant 1, \bar{\mu} \in \mathscr{M}(\bar{X}(G) \backslash X(G), S)\right\}
\end{aligned}
$$

где $\mathscr{M}(\bar{X}(G) \backslash X(G), S)$ - множество $S$-инвариантных борелевских вероятностных мер $\bar{\mu}$ на $\bar{X}(G)$, для которых $\bar{\mu}(X(G))=0$; если, кроме того, матрица $A_{f_{0}}$, əде $f_{0} \equiv 0$, допустима (см. $\left.\S 2\right)$, то $\partial P_{f}(0)=\left\{c \mu^{f}+(1-c) \delta_{\infty}\right.$, $0 \leqslant c \leqslant 1\}$.

ДокАЗАТЕльСтво. Так как функция $g \mapsto \partial P_{f}(g)$ непрерывна, множество $\partial P_{f}(0)$ непусто и вьпукло (см. [47, гл. 4]). Из определения 5.3 вытекает, что если $\nu \in \partial P_{f}(0)$ и $g \in C(\bar{X}(G))$, то

$$
t \nu(g) \leqslant P_{f}(t g)-P_{f}(0), \quad t \in \mathbb{R} .
$$

Деля на $t$ и переходя к пределу при $t \rightarrow+0$ и при $t \rightarrow-0$, получаем

$$
\omega_{f}^{-}(g) \leqslant \nu(g) \leqslant \omega_{f}^{+}(g) .
$$

В силу леммы 5.6 это означает, что если $g=\mathbf{1}_{\bar{C}}$, где $C \subset X(G)$ - произвольный сплошной цилиндр, то в случае, когда матрица $A_{f}$ невозвратна или нуль-возвратна, $\nu(g)=0$, а в случае, когда $A_{f}$ устойчиво-положительна, $\nu(g)=\mu^{f}(C)$. Отсюда видно, что в первом случае мера $\nu$ равна нулю на любом цилиндре $C \subset X(G)$ с одноточечным носителем и даже на его замькании. Следовательно, $\nu$ сосредоточена в точке $x^{\infty}$ (согласно предложению 5.1 в рассматриваемом случае $x^{\infty} \in \bar{X}(G)$ ), а так как по лемме $5.6 \nu$ - вероятностная мера, она совпадает с $\delta_{\infty}$. Во втором случае мера $\nu$ сосредоточена на $X(G)$. Действительно, пусть $n \in \mathbb{Z}, w \in \overline{\mathbb{N}}$ и $\bar{C}(n, w)=\{x \in$ $\left.\bar{X}(G): x_{n}=w\right\}$. Если $w \neq \infty$, т.е. $w \in V(G)$, то, очевидно, $\bar{C}(n, w)$ есть замыкание шилиндра $C(n, w)=\left\{x \in X(G): x_{n}=w\right\}$, и по доказанному $\nu(\bar{C}(n, w))=$ 
$\mu^{f}(C(n, w))$. Суммируя по $w \in V(G)$, с учетом того, что $\nu$ и $\mu^{f}$ - вероятностные меры, получим $\nu(\bar{C}(n, \infty))=0, n \in \mathbb{Z}$. Но тогда $\nu(\bar{X}(G) \backslash X(G))=0$, так как $\bar{X}(G) \backslash X(G) \subset \bigcup_{n \in \mathbb{Z}} \bar{C}(n, \infty)$. Следовательно, $\nu(\bar{C})=\mu^{f}(C)$ для любого сплошного цилиндра $C \subset X(G)$. Поскольку борелевская мера на $X(G)$ определяется своими значениями на таких цилиндрах, мы заключаем, что $\nu=\mu^{f}$.

Переходя к случаю, когда матрища $A_{f}$ неустойчиво-положительна, вычислим сначала $\partial P_{f}(g)$ при некоторых $g$, близких к нулю. Возьмем произвольные $v \in V(G)$, $t \in \mathbb{R}$ и обозначим через $\chi$ и $\bar{\chi}$ характеристические функции цилиндров $C(v)$ и $\bar{C}(v)$ соответственно. Из п. 5 леммы 5.2 с учетом того, что $f+t \chi \in \mathscr{F}_{0}^{1}(G)$, легко вывести соотношение

$$
P_{f}(t \bar{\chi}+g)=P_{f+t \chi}(g), \quad g \in C(\bar{X}(G)),
$$

вследствие которого

$$
\partial P_{f}(t \bar{\chi})=\partial P_{f+t \chi}(0), \quad t \in \mathbb{R} .
$$

Таким образом, вычисление субдифференциала функции $P_{f}$ в точке $t \bar{\chi}$ свелось к вычислению субдифференциала функции $P_{f+t \chi}$ в нуле. Но последний нам, по существу, уже известен. В самом деле, из определения матрицы $A_{f+t \chi}$ видно, что при всех $t \in \mathbb{R}$

$$
R_{v v}^{0}\left(A_{f+t \chi}\right)=R_{v v}^{0}\left(A_{f}\right), \quad \varphi_{v v}^{0}\left(A_{f+t \chi}, z\right)=e^{t} \varphi_{v v}^{0}\left(A_{f}, z\right), \quad|z|<R_{v v}^{0}\left(A_{f}\right) .
$$

По предположению матрица $A_{f}$ неустойчиво-положительна. Поэтому матрица $A_{f+t \chi}$ при $t<0$ невозвратна, а при $t>0$ - устойчиво-положительна (см. §2). По доказанному в первом случае $\partial P_{f}(t \bar{\chi})=\left\{\delta_{\infty}\right\}$, а во втором случае $\partial P_{f}(t \bar{\chi})=\left\{\mu^{f+t \chi}\right\}$ (см. (5.27)).

Теперь воспользуемся общей теоремой выпуклого анализа (см., например, [46]), которая утверждает, что если $F$ - выпуклая функция, определенная на банаховом пространстве $H,\left\{h_{n}, n \in \mathbb{Z}\right\}$ - последовательность элементов этого пространства, сходяшаяся к $h, \nu_{n} \in \partial F\left(h_{n}\right), n \in \mathbb{N}$, причем $\nu_{n} \rightarrow \nu$ при $n \rightarrow \infty$, то $\nu \in \partial F(h)$. Беря $t=t_{n}$, где $t_{n} \rightarrow-0$, получаем из этой теоремы включение $\delta_{\infty} \in \partial P_{f}(0)$. Покажем, что если $t \rightarrow+0$, то $\mu^{f+t \chi}$ сходится к $\mu^{f}$.

Вследствие компактности пространства $\bar{X}(G)$ семейство мер $\mu^{f+t} \chi, t \geqslant 0$, pacсматриваемых как меры на $\bar{X}(G)$, слабо компактно. Пусть $\nu$-произвольная предельная точка при $n \rightarrow \infty$ последовательности $\mu^{f+t_{n} \chi}$, где $t_{n}>0$ при всех $n$ и $t_{n} \rightarrow 0$ при $n \rightarrow \infty$. Не ограничивая обшности, будем считать, что $\mu^{f+t_{n} \chi} \rightarrow \nu$. Надо убедиться, что $\nu=\mu^{f}$. По теореме $4.6 \mu^{f+t} \chi$ при любом $t>0$ - стационарная марковская мера, сосредоточенная на $X(G)$, причем ее параметры определяются соотношением (4.2). Возьмем произвольную вершину $v \in V(G)$ и произвольный $v$-цилиндр $C \subset X(G)$ (см. (5.10)). Если у него одноточечный носитель, то $C=C(k, v), k \in \mathbb{Z}$. В противном случае $C=C(k, \gamma), k \in \mathbb{Z}, \gamma \in \Gamma_{v v}(G, l), l \geqslant 1$. Из (4.2), (4.12) и определения матришы $A_{f+t \chi}$ с помошью простой выкладки получаем

$$
\mu^{f+t \chi}(C)= \begin{cases}{\left[R\left(A_{f+t \chi}\right) D_{v}\left(A_{f+t \chi}\right)\right]^{-1},} & C=C(k, v), \\ {\left[R\left(A_{f+t \chi}\right)\right]^{l-1} Q\left(A_{f}, \gamma\right) e^{t m(C)} / D_{v}\left(A_{f+t \chi}\right),} & C=C(k, \gamma),\end{cases}
$$

где $m(C)$ - число простых $v$-циклов, произведение которых есть $\gamma$ (см. определение 1.1). 
В силу (5.28)

$$
D_{v}\left(A_{f+t \chi}\right)=\left.\frac{d}{d \tau} \varphi_{v v}^{0}\left(A_{f+t \chi}, \tau\right)\right|_{\tau=R\left(A_{f+t \chi}\right)}=\left.e^{t} \frac{d}{d \tau} \varphi_{v v}^{0}\left(A_{f}, \tau\right)\right|_{\tau=R\left(A_{f+t \chi}\right)} .
$$

Так как матрица $A_{f+t \chi}$ устойчиво-положительна, число $R\left(A_{f+t \chi}\right)$ определяется равенством

$$
\varphi_{v v}^{0}\left(A_{f}, R\left(A_{f+t \chi}\right)\right)=e^{-t}
$$

(см. лемму 2.6), откуда вытекает, что

$$
\lim _{t \rightarrow+0} R\left(A_{f+t \chi}\right)=R\left(A_{f}\right), \quad \lim _{t \rightarrow+0} D_{v}\left(A_{f+t \chi}\right)=D_{v}\left(A_{f}\right) .
$$

Возврашаясь к (5.29), получаем

$$
\lim _{t \rightarrow+0} \mu^{f+t \chi}(\bar{C})=\lim _{t \rightarrow+0} \mu^{f+t \chi}(C)=\mu^{f}(C),
$$

где $\bar{C}$ - замькание множества $C$ в $\bar{X}(G)$. Поскольку, как уже отмечалось, характеристическая функция множества $\bar{C}$ непрерьвна, из сходимости $\mu^{f+t_{n} \chi} \rightarrow \nu$ вытекает, что $\nu(\bar{C})=\mu^{f}(C)$. Теперь, пользуясь леммой 5.8 (при $\mu=\mu^{f}, \alpha_{v} \equiv 1$ ), моментально приходим к равенству $\mu=\nu$. Тем самьм доказано, что $\mu^{f} \in \partial P_{f}(0)$. Поскольку мы уже знаем, что множество $\partial P_{f}(0)$ содержит $\delta_{\infty}$ и вьпукло, это приводит к первому из включений (5.25). Перейдем ко второму.

Очевидно, всякую меру $\nu \in \mathscr{M}(\bar{X}(G), S)$ можно однозначным образом представить в виде $\nu=c \nu^{\prime}+(1-c) \nu^{\prime \prime}$, где

$$
0 \leqslant c \leqslant 1, \quad \nu^{\prime}, \nu^{\prime \prime} \in \mathscr{M}(\bar{X}(G), S), \quad \nu^{\prime}(X(G))=1, \quad \nu^{\prime \prime}(X(G))=0
$$

Если $\nu \in \partial P_{f}(0)$ и $g=\chi_{\bar{C}}$, где $C \subset X(G)$ - сплошной цилиндр, то в силу $(5.26)$ и п. 3 леммы 5.6

$$
\mu^{f}(C)=\omega_{f}^{+}(g) \geqslant \nu(\bar{C}) \geqslant \nu(C)=c \nu^{\prime}(C) .
$$

При $c \neq 0$ отсюда следует абсолютная непрерывность меры $\nu^{\prime}$ относительно $\mu^{f}$, а так как обе меры $S$-инвариантны и $\mu^{f}$ обладает свойством эргодичности (см. замечание 4.8), мы приходим к выводу, что $\nu^{\prime}=\mu^{f}$. Тем самым второе из включений (5.25) также доказано.

Осталось рассмотреть случай, когда матрица смежности графа $G$ допустима. Из определения метрики $d_{2}$ следует, что тогда во всякой последовательности $x \in \bar{X}(G)$, содержащей символ $\infty$, все места справа или слева от него занимает тот же самый символ. Отсюда нетрудно вывести, что на $\bar{X}(G) \backslash X(G)$ может быть сосредоточена только одна $S$-инвариантная мера, а именно, $\delta_{\infty}$. Тем самым, $\nu^{\prime \prime}=\delta_{\infty}$ (согласно п. 1 предложения 5.1 в нашем случае $\left.x^{\infty} \in \bar{X}(G)\right)$. Теорема доказана. 
5.10. ЗАмечАнИЕ. Из теоремы 5.9 вытекает, что функция $P_{f}$, где $f \in \mathscr{F}_{0}^{1}(G)$, дифференщируема по Гато в нуле во всех случаях, кроме случая неустойчивой положительности матрицы $A_{f}$. Можно показать, однако, что ни в одном из этих случаев $P_{f}$ не обладает более сильным свойством дифференцируемости по Фреше.

Еше одно применение теоремы 5.9 связано с описанием равновесных мер на пространстве $\bar{X}(G)$, отвечаюших функциям $\bar{f}$, ограничение которых на $X(G)$ принадлежит $\mathscr{F} 1(G)$. Пусть $\bar{f}$ - любая такая функция и $f=\left.\bar{f}\right|_{X(G)}$. Согласно теореме 1.5 и вариационному принципу (1.5)

$$
P^{\mathrm{int}}(X(G), S, f)=P(\bar{X}(G), S, \bar{f})=\sup _{\mu \in \mathscr{M}(\bar{X}(G), S)}\left[h_{\mu}(S)+\int_{\bar{X}(G)} \bar{f} d \mu\right] .
$$

Обозначим через $\mathscr{E} Q(\bar{f})$ множество $\bar{f}$-равновесных состояний, т.е. таких мер $\mu \in$ $\mathscr{M}(\bar{X}(G), S)$, для которых

$$
h_{\mu}(S)+\int_{\bar{X}(G)} \bar{f} d \mu=P(\bar{X}, S, \bar{f}) .
$$

5.11. Теорема. Пусть функиия $\bar{f} \in C(\bar{X}(G))$ удовлетворяет условиям $f \in \mathscr{F}_{0}^{1}(G), R\left(A_{f}\right)>0$, где $f=\left.\bar{f}\right|_{X(G)}$. Тогда:

1) если матрица $A_{f}$ невозвратна или нуль-возвратна, то

$$
\mathscr{E} \mathscr{Q}(\bar{f})= \begin{cases}\varnothing & \text { npu } \bar{f}\left(x^{\infty}\right)<P^{\operatorname{int}}(X(G), S, f), \\ \left\{\delta_{\infty}\right\} & \text { npu } \bar{f}\left(x^{\infty}\right)=P^{\operatorname{int}}(X(G), S, f) ;\end{cases}
$$

2) если матрица $A_{f}$ устойчиво-положительна, то $\mathscr{E}(\bar{f})=\left\{\mu^{f}\right\}$;

3) если матрица $A_{f}$ неустойчиво-положительна, то

$$
\mathscr{E} \mathscr{Q}(\bar{f})= \begin{cases}\left\{\mu^{f}\right\} & \text { npu } \bar{f}\left(x^{\infty}\right)<P^{\operatorname{int}}(X(G), S, f), \\ \left\{c \mu^{f}+(1-c) \delta_{\infty}, 0 \leqslant c \leqslant 1\right\} & \text { nрu } \bar{f}\left(x^{\infty}\right)=P^{\operatorname{int}}(X(G), S, f) .\end{cases}
$$

5.12. ЗАМЕЧАнИЕ. Из (5.32) и очевидного равенства $h_{\delta_{\infty}}(S)=0$ вытекает, что во всех случаях $\bar{f}\left(x^{\infty}\right) \leqslant P^{\operatorname{int}}(X(G), S, f)$.

ДокаЗАТельСтво теоРемы 5.11. Пусть $\bar{g} \in C(\bar{X}(G)), g=\left.\bar{g}\right|_{X(G)}$ и $\bar{\mu} \in \mathscr{E} \mathscr{Q}(\bar{f})$. По теореме 1.5 (см. также (1.5))

$$
\begin{aligned}
P_{f}(\bar{g}) & =P^{\operatorname{int}}(X(G), S, f+g)=P(\bar{X}(G), S, \bar{f}+\bar{g}) \\
& =\sup _{\mu \in \mathcal{M}(\bar{X}(G), S)}\left[h_{\mu}(S)+\int_{\bar{X}(G)} \bar{f} d \mu+\int_{\bar{X}(G)} \bar{g} d \mu\right] \\
& \geqslant h_{\mu}(S)+\int_{\bar{X}(G)} \bar{f} d \bar{\mu}+\int_{\bar{X}(G)} \bar{g} d \bar{\mu}=P_{f}(0)+\int_{\bar{X}(G)} \bar{g} d \bar{\mu},
\end{aligned}
$$

откуда видно, что $\bar{\mu}$ - субградиент функции $P_{f}$ в нуле (см. определение 5.3). Тем самым, в условиях теоремы $5.11 \mathscr{E} \mathscr{Q}(\bar{f}) \subset \partial P_{f}(0)$. Применяя пп. 1,2 теоремы 5.9, 
получаем отсюда утверждения 1) и 2). Для доказательства утверждения 3) заметим, что в силу (5.32) и теоремы 4.4 условие $R\left(A_{f}\right)>0$ влечет за собой неравенство $h(\bar{X}(G), S)<\infty$ (так как функщия $\bar{f}$ ограничена), из которого, в частности, вытекает, что матрица $A_{f_{0}}$ (здесь, как и раньше, $\left.f_{0}(x) \equiv 0, x \in X(G)\right)$ допустима. При этом условии множество $\partial P_{f}(0)$ полностью описано в теореме 5.9 во всех случаях, включая интересующий нас сейчас случай неустойчиво-положительной матрицы $A_{f}$. Теперь утверждение 3) теоремы 5.11 получается из п. 3 теоремы 5.9 с помощью следующего простого замечания: если мера $\mathscr{E} Q(\bar{f})$ есть выпуклая комбинация с ненулевыми коэффициентами двух взаимно сингулярных мер $\mu^{\prime}, \mu^{\prime \prime} \in \mathscr{M}(\bar{X}(G), S)$, то $\mu^{\prime}, \mu^{\prime \prime} \in \mathscr{E} \mathscr{Q}(\bar{f})$; это вытекает из свойств энтропии [30]. Теорема доказана.

Теорема 5.11 нуждается в следуюшем естественном дополнении.

5.13. ПРЕДЛОЖЕНИЕ. Все случаи, упомянутые в теореме 5.11, реализуемьи, m.е. для каждого из них найдется соответствующая пара $(G, f)$.

Прежде чем доказывать это предложение, введем один важньй класс связных ориентированных графов, который уже неявно присутствовал в некоторых конструкциях предыдуших параграфов и еше встретится ниже.

5.14. ОПРеДЕЛЕниЕ. Пусть $G$ - ориентированньй граф̆, построенный по последовательности цельх чисел $l_{k}, k=1,2, \ldots$, следуюшим образом: вершины $v \in V(G)$ графа $G$ отождествляются с парами целых чисел $(0,0),(k, l), k=1,2, \ldots, 1 \leqslant l \leqslant$ $l_{k}-1$, а ребра - с парами вершин

$$
((0,0),(k, 1)),((k, l),(k, l+1)),\left(\left(k, l_{k}-1\right),(0,0)\right), k=1,2, \ldots, 1 \leqslant l \leqslant l_{k}-2 .
$$

Последовательность вершин $(0,0),(k, 1), \ldots,\left(k, l_{k}-1\right),(0,0)$ назовем лепестком с номером $k$, а сам только что описанньй граф - лепестковым графом с длинами лепестков $l_{1}, l_{2}, \ldots$ и центральной вершиной $(0,0)$.

Ясно, что лепестковый граф $G$ определяется не самой последовательностью длин его лепестков, а тем, сколько раз в ней встречается каждое натуральное число, т.е. последовательностью $\left\{N_{l}(G), l \geqslant 2\right\}$, где $N_{l}(G) \in \mathbb{Z}^{+} \cup\{\infty\}$ - число лепестков длины $l$. Эту вторую последовательность будем назьвать распределением графа $G$.

ДокАЗАТЕЛЬСТВо ПРЕДЛОЖЕНИЯ 5.13. Рассмотрим лепестковый граф $G$, распределение которого имеет вид $N_{l}(G)=1$ при $l \geqslant 3, N_{2}(G)=0$, и зададим функцию $f_{\alpha} \in \mathscr{F}_{0}^{1}(G)$, зависящую от действительного параметра $\alpha$, условием $f_{\alpha}(x)=$ $-(\alpha / l) \ln l$, если $x_{0}, x_{1}$ принадлежат лепестку длины $l$. Очевидно, $f_{\alpha}$ можно продолжить до функции $\bar{f}_{\alpha} \in C(\bar{X}(G))$, причем $\bar{f}_{\alpha}\left(x^{\infty}\right)=0$ (из определения $G$ непосредственно вытекает, что $\left.x^{\infty} \in \bar{X}(G)\right)$. Пусть $v$ - центральная вершина графа $G$. По построению

$$
\varphi_{v v}^{0}\left(A_{\bar{f}_{\alpha}}, z\right)=\sum_{n=3}^{\infty} \frac{z^{n}}{n^{\alpha}}
$$

откуда видно, что $R_{v v}^{0}\left(A_{f_{\alpha}}\right)=1$ при любом $\alpha$. Если $\alpha \leqslant 2$ таково, что $\sum_{n=3}^{\infty} n^{-\alpha} \leqslant 1$ (легко видеть, что такие $\alpha$ сушествуют), то $\varphi_{v v}^{0}\left(A_{f_{\alpha}}, 1\right) \leqslant 1$ и ряд, полученный из 
(5.33) почленным дифференцированием, в точке $z=1$ расходится. Это значит, что матрица $A_{f_{\alpha}}$ либо невозвратна (когда последние два неравенства - строгие), либо нуль-возвратна (когда имеют место равенства). В обоих случаях $P^{\operatorname{int}}\left(X(G), S, f_{\alpha}\right)=0$ (см. теорему 4.4) и, значит,

$$
\bar{f}_{\alpha}\left(x^{\infty}\right)=P^{\operatorname{int}}\left(X(G), S, f_{\alpha}\right)=0 .
$$

Чтобы доказать совместимость соотношения (5.34) и неустойчивой положительности, возьмем в предыдущей конструкции параметр $\alpha>2$, для которого $\sum_{n=3}^{\infty} n^{-\alpha}$ $\leqslant 1$, и подберем $\beta>0$ так, чтобы выполнялось равенство $e^{\beta} \sum_{n=3}^{\infty} n^{-\alpha}=1$. Пусть $\chi=\mathbf{1}_{C(v)}$ и $f_{\alpha \beta}=f_{\alpha}+\beta \chi$. Тогда

$$
R_{v v}^{0}\left(A_{f_{\alpha \beta}}\right)=R\left(f_{\alpha \beta}\right)=1, \quad \varphi_{v v}^{0}\left(A_{f_{\alpha, \beta}}, 1\right)=1,\left.\quad \frac{d}{d t} \varphi_{v v}^{0}\left(A_{f_{\alpha, \beta}}, t\right)\right|_{t=1}<\infty
$$

откуда видно, что матрица $A_{f_{\alpha, \beta}}$ неустойчиво-положительна (см. следствие 2.10). Так как, очевидно, $f_{\alpha, \beta}$ продолжается до функции $\bar{f}_{\alpha, \beta} \in C(\bar{X}(G))$ и $\bar{f}_{\alpha, \beta}\left(x^{\infty}\right)=0$, мы получаем $\bar{f}_{\alpha, \beta}\left(x^{\infty}\right)=P^{\text {int }}\left(X(G), S, f_{\alpha, \beta}\right)$.

Теперь возьмем лепестковый граф $G^{1}$, для которого $N_{l}\left(G^{1}\right)=2^{l}$ при $l \geqslant 3$ и $N_{2}\left(G^{1}\right)=0$. Снова обозначив через $v$ центральную вершину и определив функцию $f_{\alpha}^{1}: X\left(G^{1}\right) \rightarrow \mathbb{R}$ точно так же, как вьше была определена функция $f_{\alpha}$, получим тождество

$$
\varphi_{v v}^{0}\left(A_{f_{\alpha}^{1}}, z\right) \equiv \varphi_{v v}^{0}\left(A_{f_{\alpha}}, 2 z\right) .
$$

Из него следует, что $R_{v v}^{0}\left(A_{f_{\alpha}^{1}}\right)=1 / 2$ и что матрица $A_{f_{\alpha}^{1}}$ невозвратна и нуль-возвратна при тех же самых $\alpha$, при которых соответствуюшими свойствами обладает матрица $A_{f_{\alpha}}$. При этих $\alpha$ вьполняется равенство $R\left(A_{f_{\alpha}}\right)=1 / 2$ и в то же время, аналогично предыдущему, $f_{\alpha}^{1}$ продолжается до функщии $\bar{f}_{\alpha}^{1} \in C\left(\bar{X}\left(G^{1}\right)\right)$, равной нулю в точке $x^{\infty} \in \bar{X}\left(G^{1}\right)$. В результате мы приходим к неравенству $\bar{f}_{\alpha}^{1}\left(x^{\infty}\right)<P\left(X\left(G^{1}\right), S, f_{\alpha}^{1}\right)$.

Наконец, дословно повторив рассуждения, связанные с введением функции $f_{\alpha, \beta}$, мы получим функцию $f_{\alpha, \beta}^{1}$, для которой матрица $A_{f_{\alpha, \beta}^{1}}$ неустойчиво-положительна и вьполняется неравенство $\bar{f}_{\alpha \beta}^{1}\left(x^{\infty}\right)<P^{\mathrm{int}}\left(X\left(G^{1}\right), S, f_{\alpha \beta}^{1}\right)$. Тем самым, рассмотрены все случаи, встречающиеся в утверждениях 1) и 3) теоремы 5.11. Очевидно, утверждение 2) в построении каких-либо примеров не нуждается. Предложение полностью доказано.

5.15. ЗАмечание. Результаты, изложенные в этом параграфе, позволяют без особых усилий вьвести из вариационного принципа, сформулированного в теореме 4.4 , теорему Заргаряна 1.5. Действительно, в случае бесконечной топологической энтропии $P(X(G), S, 0)$ утверждение теоремы 1.5 очевидно, так как речь идет об ограниченных функциях $f$. Далее, вариационньй принцип

$$
\sup _{\mu \in \mathscr{E}(X(G), S)} P(X(G), f, \mu)=P^{\operatorname{int}}(X(G), S, f),
$$

сформулированньй в теореме 4.4 для функций $f \in \mathscr{F}_{0}^{1}(G)$, с помощью преобразований редукции и сдвига моментально обобщается на все ограниченные локальные функции 
(см. определение 1.10). При $P(X(G), S, 0)<\infty$ обе части равенства (5.35) имеют смысл для любой ограниченной непрерывной функции $f: X(G) \rightarrow \mathbb{R}$ и непрерьвны по $f$ в метрике пространства $C(X(G))$ (см. предложение 4.3). Поэтому (5.35) обобщается на все равномерно непрерьвные ограниченные $f$ (поскольку их можно аппроксимировать в $C(X(G))$ локальньми функциями). Остается доказать, что если $\bar{f} \in C(\bar{X}(G))$ и $f=\left.\bar{f}\right|_{X(G)}$, то

$$
\sup _{\mu \in \mathscr{E}(X(G), S)} P(X(G), f, \mu)=\sup _{\mu \in \mathscr{E}(\bar{X}(G), S)} P(\bar{X}(G), \bar{f}, \mu),
$$

где $\mathscr{E}(\bar{X}(G), S)$ - множество эргодических (относительно $S$ ) вероятностных борелевских мер на $\bar{X}(G)$ (см. (1.5)). Но при $P(X(G), S, 0)<\infty$ матрица $A_{f_{0}}$, где $f_{0} \equiv 0$, допустима, $x^{\infty} \in \bar{X}(G)$ (см. п. 1 предложения 5.1) и $\mathscr{E}(\bar{X}(G), S) \backslash \mathscr{E}(X(G), S)=\left\{\delta_{\infty}\right\}$ (мы уже отмечали последнее обстоятельство при доказательстве теоремы 5.9). Так как $h_{\delta_{\infty}}=0$, равенство (5.36) будет доказано, если найдется последовательность мер $\mu_{n} \in \mathscr{M}(X(G), S)$, сходяшаяся к $\delta_{\infty}$. Такую последовательность нетрудно указать в классе дискретных $S$-инвариантных мер (эти меры сосредоточены на множестве периодических последовательностей; им посвящен $\S 7$ данной статьи).

5.16. ЗАмЕчАниЕ. Еще одно применение основных результатов этого параграфа касается множества $f$-равновесных мер для $f \in \mathscr{F}_{0}^{1}$, описанного без доказательства в теореме 4.6. Воспользовавшись тем, что из вариационного принципа, содержашегося в п. 5 теоремы 5.2 , вытекает включение $\mathscr{E} \mathscr{Q}(f) \subseteq \partial P_{f}(0)$, и действуя далее в духе леммы 5.6 и теоремы 5.9 , можно изложить доказательство теоремы 4.6 в важном частном случае, когда топологическая энтропия $P^{\operatorname{int}}(X(G), f, 0)$ конечна и, значит, для определения функционала $P(X(G), f, \cdot)$ не нужна регуляризация, гораздо короче, чем это делается в общем случае [25].

\section{§. Асимптотически-равновесные меры. Сходимость равновесных мер, отвечающих конечным подцепям счетной СЦМ}

В этом параграфе обобщаются и усиливаются результаты, опубликованные без доказательства в [35]. Как и в предыдуших параграфах, будем рассматривать СЦМ $(X(G), S)$, где $G$ - связный ориентированный граф со счетным множеством вершин $V(G)$ и $S$ - сдвиг на пространстве $X(G)$ его двусторонне-бесконечных путей. Говоря о сходимости или компактности семейств мер, сосредоточенных на $X(G)$, будем по-прежнему считать эти меры заданными на компактном пространстве $\bar{X}(G)$.

6.1. ОПРедЕлЕниЕ. Назовем последовательность $S$-инвариантных вероятностных борелевских мер $\mu_{n}$, определенных на $X(G)$, асимптотически равновесной относительно функции $f \in \mathscr{F}_{0}^{1}(G)$ (короче-асимптотически $f$-равновесной), если

$$
\lim _{n \rightarrow \infty} P\left(X(G), f, \mu_{n}\right)=P^{\text {int }}(X(G), S, f) .
$$

Естественный пример асимптотически $f$-равновесной последовательности возникает следуюшим образом. Пусть $f \in \mathscr{F}_{0}^{1}(G),\left\{G_{n}\right\}$ - исчерпьвающая последовательность конечных связных подграфов графа $G$ (см. определение 3.9) и $f_{n}=\left.f\right|_{X\left(G_{n}\right)}$. 
Тогда $f_{n}$-равновесные меры $\mu_{n}$ (которые можно считать заданными на $X(G)$ ) образуют асимптотически $f$-равновесную последовательность. Это непосредственно вытекает из леммы 3.10 , замечания 3.11 и теоремы 4.4.

В следующей ниже лемме используется обозначение из леммы 5.2.

6.2. ЛЕмма. Если $f \in \mathscr{F}_{0}^{1}(G)$ и меры $\mu_{n} \in \mathscr{E}_{f}(X(G), S)$ образуют асимптотически $f$-равновесную последовательность, то всякая предельная точка этой последовательности принадлежст субдифференщиалу $\partial P_{f}(0)$ (см. 5 ).

ДоКАЗАТЕЛЬСТВо. В силу (5.2) для любой функции $g \in C(\bar{X}(G))$ вьполняется неравенство

$$
\int g d \mu_{n} \leqslant P_{f}(g)-P\left(X(G), f, \mu_{n}\right), \quad n=1,2, \ldots
$$

Переходя в нем к пределу при $n \rightarrow \infty$ и учитьвая определение 6.1 , получаем

$$
\int g d \nu \leqslant P_{f}(g)-P_{f}(0)
$$

где $\nu$ - произвольная предельная точка последовательности $\left\{\mu_{n}\right\}$. Тем самым, $\nu \in$ $\partial P_{f}(0)$ (см. определение 5.3). Лемма доказана.

Из этой леммы и теоремы 5.9 немедленно вытекает следующее утверждение.

6.3. ТеОрема. Пусть $f \in \mathscr{F}_{0}^{1}(G), R\left(A_{f}\right)>0,\left\{\mu_{n}\right\}-$ произвольная асимптотически $f$-равновесная последовательность мер из $\mathscr{E}_{f}(X(G), S)$ и $L\left(\left\{\mu_{n}\right\}\right)$ мно сество ее предельных точек. Тогда:

1) если матрица $A_{f}$ невозвратна или нуль-возвратна, то $L\left(\left\{\mu_{n}\right\}\right)=\left\{\delta_{\infty}\right\}$;

2) если матрица $A_{f}$ устойчиво-положительна, то $L\left(\left\{\mu_{n}\right\}\right)=\left\{\mu^{f}\right\}$;

3) если матрича $A_{f}$ неустойчиво-положстельна, а матрица $A_{f_{0}}$, где $f_{0} \equiv 0$, допустима, то

$$
L\left(\left\{\mu_{n}\right\}\right) \subset\left\{c \mu^{f}+(1-c) \delta_{\infty}, 0 \leqslant c \leqslant 1\right\} .
$$

Если $f \in \mathscr{F}_{0}^{1}(G)$, то упомянутая выше последовательность $f_{n}$-равновесных мер, построенная по $f$ и исчерпьваюшей последовательности конечных связных подграфов графа $G$, удовлетворяет условиям теоремы 6.3 , так как при всех $n$ множество $X\left(G_{n}\right)$, на котором сосредоточена мера $\mu_{n}$, компактно и функция $f$ на нем ограничена. Тогда согласно первьм двум утверждениям этой теоремы $\mu_{n} \rightarrow \delta_{\infty}$, если матрища $A_{f}$ невозвратна или нуль-возвратна (т.е. не сушествует $f$-равновесной меры, см. теорему 4.6$),$ и $\mu_{n} \rightarrow \mu^{f}$, если матрица $A_{f}$ устойчиво-положительна (вследствие чего $f$-равновесная мера $\mu^{f}$ существует). Для случая неустойчиво-положительной матрицы $A_{f}$, когда $f$-равновесная мера также сушествует, теорема 6.3 дает менее точную информацию, да и то при дополнительном условии допустимости матрицы $A_{f_{0}}$. Теорема, которую мы сейчас сформулируем, относится (в отличие от теоремы 6.3) лишь к асимптотически-равновесным последовательностям мер из рассматриваемого класса, но зато не делает различия между устойчиво-положительными и неустойчиво-положительными матрицами $A_{f}$. K тому же она не содержит никаких ограничений на матрицу $A_{f_{0}}$. Используемое ниже обозначение $D_{v}(\cdot)$ введено в $\S 4$ (см. (4.7)). 
6.4. ТЕОремА. Пусть $\left\{G_{n}\right\}$ - исчерпьвающая последовательность конечных связных подграфов графа $G, f \in \mathscr{F}_{0}^{1}(G), A=A_{f}$ - матрица Больимана, отвечающая функции $f$, и $A_{n}-$ матрица Больцмана, отвечающая функции $f_{n}=\left.f\right|_{X\left(G_{n}\right)}$. Предположим, что $R(A)>0$ и существует $f$-равновесная мера $\mu=\mu^{f}$. Тогда для сходимости последовательности $f_{n}$-равновесных мер $\mu_{n}=\mu^{f_{n}}$ необходимо и достаточно, чтобы нашлась вериина $v \in V(G)$, для которой существует конечный или бесконечный предел

$$
\lim _{n \rightarrow \infty} D_{v}\left(A_{n}\right)=D\left(\left\{A_{n}\right\}, v\right)
$$

если это условие вьполнено, то (6.1) имеет место для всех $v \in V(G)$ и

$$
\lim _{n \rightarrow \infty} \mu_{n}=\alpha \mu+(1-\alpha) \delta_{\infty}
$$

$2 \partial e$

$$
\alpha=\frac{D_{v}(A)}{D\left(\left\{A_{n}\right\}, v\right)} \in[0,1]
$$

не зависит от $v$ (при $\alpha=1$ (6.2) считается выполненным и в том случае, когда $\left.x^{\infty} \notin \bar{X}(G)\right)$.

ДоКАЗАТЕЛЬСТВо. В приводимых ниже рассуждениях мы будем неоднократно использовать явньй вид мер $\mu_{n}$ и $\mu$, опираясь на теорему 4.6 и соотношения (4.2), (4.12), которые, однако, каждьй раз упоминаться не будут.

Вначале докажем необходимость условия (6.1). Пусть $\nu=\lim _{n \rightarrow \infty} \mu_{n}$ и $v \in V(G)$. Как уже отмечалось в $\S 5$, характеристическая функция замькания $\bar{C}(v)$ цилиндра $C(v)$ непрерьвна. Поэтому

$$
\lim _{n \rightarrow \infty} \mu_{n}(C(v))=\lim _{n \rightarrow \infty} \mu_{n}(\bar{C}(v))=\mu(\bar{C}(v))
$$

(первое равенство вытекает из того, что мера $\mu$ сосредоточена на $X(G)$ ). Но

$$
\mu_{n}(C(v))=\frac{1}{R\left(A_{n}\right) D_{v}\left(A_{n}\right)}
$$

и, так как $\lim _{n \rightarrow \infty} R\left(A_{n}\right)=R(A)$ (см. лемму 3.10$)$, причем $R(A)>0$, мы получаем

$$
D\left(\left\{A_{n}\right\}, v\right)=\lim _{n \rightarrow \infty} D_{v}\left(A_{n}\right)=\frac{1}{R(A) \nu(\bar{C}(v))}, \quad v \in V(G)
$$

(здесь, как обычно, $1 / 0=\infty$ ). Тем самым, не только доказана необходимость сушествования предела (6.1) для сходимости последовательности мер $\mu_{n}$, но и найдена связь этого предела с предельной мерой.

Чтобы доказать остальные утверждения теоремы, возьмем произвольную предельную точку $\nu$ последовательности $\left\{\mu_{n}\right\}$ (такие точки существуют вследствие компактности $\bar{X}(G))$ и предположим, что $\mu_{n_{i}} \rightarrow \nu$. Очевидно, последовательность $\left\{G_{n_{i}}\right\}$, 
как и $\left\{G_{n}\right\}$, является исчерпываюшей. Тогда по доказанному для любой вершины $v \in V(G)$ существует предел

$$
\lim _{i \rightarrow \infty} D_{v}\left(A_{n_{i}}\right)=D\left(\left\{A_{n_{i}}\right\}, v\right)
$$

(cp. (6.4)), причем

$$
\nu(\bar{C}(v))=\frac{1}{R(A) D\left(\left\{A_{n_{i}}\right\}, v\right)} .
$$

Всякий сплошной $v$-цилиндр $C \subset X(G)$, носитель которого содержит больше одной точки, имеет вид $C(k, \gamma), k \in \mathbb{Z}, \gamma \in \Gamma_{v v}(G)$, а потому при каждом $n$

$$
\mu_{n}(C)=\mu_{n}(C(k, \gamma))=Q\left(A_{n}, \gamma\right)\left[R\left(A_{n}\right)\right]^{l(\gamma)-1} / D_{v}\left(A_{n}\right)
$$

где $l(\gamma)$ - длина $v$-цикла $\gamma$. Очевидно, найдется такое $n(\gamma)$, что $\gamma \in \Gamma_{v v}\left(G_{n}\right)$ при всех $n \geqslant n(\gamma)$. Следовательно, при достаточно больших $n$ в (6.7) вместо $Q\left(A_{n}, \gamma\right)$ можно писать $Q(A, \gamma)$. Отсюда с учетом $(6.5),(6.6)$ и сходимости $R\left(A_{n}\right)$ к $R(A)$ получаем

$$
\begin{aligned}
\nu(\bar{C}(k, \gamma)) & =\lim _{i \rightarrow \infty} \mu_{n_{i}}(\bar{C}(k, \gamma))=\frac{Q(A, \gamma)[R(A)]^{l(\gamma)-1}}{D\left(\left\{A_{n_{i}}\right\}, v\right)} \\
& =Q(A, \gamma)[R(A)]^{l(\gamma)} \nu(\bar{C}(v))=\frac{\nu(\bar{C}(v)) \mu^{f}(C(\gamma))}{\mu^{f}(C(v))}
\end{aligned}
$$

Значит, для любого $v$-цилиндра $C$ с \# $\operatorname{supp}(C)>1$ имеет место равенство

$$
\nu(\bar{C})=\frac{\nu(\bar{C}(v))}{\mu^{f}(C(v))} \mu^{f}(C), \quad v \in V(G) .
$$

Непосредственно убеждаемся в его справедливости и для $C=C(v)$, а затем (пользуясь $S$-инвариантностью мер $\mu^{f}$ и $\left.\nu\right)$ - для всех цилиндров $C$ с \# $\operatorname{supp}(C)=1$. Теперь лемма 5.8, в которой надо положить $\mu=\mu^{f}, \alpha_{v}=\nu(\bar{C}(v)) / \mu^{f}(C(v))$, позволяет утверждать, что величина

$$
\alpha=\frac{\nu(\bar{C}(v))}{\mu^{f}(C(v))}, \quad v \in V(G),
$$

не зависит от $v, \alpha \in[0,1]$ и выполняется соотношение (6.2). Наконец, (6.3) получается из сопоставления (6.10) и (6.6) (с учетом (4.12)). Теорема доказана. 
6.5. ЗАмечАниЕ. Возврашаясь к доказательству теоремы 6.4 , заметим, что если усилить ее предположения, потребовав, чтобы матрица $A=A_{f}$ была устойчиво-положительной, то будет выполняться равенство

$$
\lim _{n \rightarrow \infty} D_{v}\left(A_{n}\right)=D_{v}(A)
$$

Если же, наоборот, предположить, что матрища $A$ не является даже положительновозвратной (и, тем самым, меры $\mu^{f}$ не существует, см. теорему 4.6), сохранив все остальные условия теоремы 6.4 , то в правой части (6.11) надо будет заменить $D_{v}(A)$ на $\infty$. Оба эти факта вытекают из соотношений (6.8) (среди которых лишь в последнем используется сушествование меры $\mu^{f}$ ) и теоремы 6.3 , но их можно доказать и непосредственно. В случае неустойчиво-положительной матрищы $A_{f}$, которьй, очевидно, не охватьвается только что сказанньм, поведение последовательности $\left\{D_{v}\left(A_{n}\right)\right\}$ оказьвается гораздо менее определенньц. Мы опишем возможные варианты этого поведения для СЦМ $(X(G), S)$, отвечаюших лепестковыц графам $G$ (см. определение 5.14). Обобшаются ли эти результаты на все графы $G$, нам не известно.

6.6. ЛЕмма. Пусть $\varphi(x)=\sum_{k=0}^{\infty} c_{k} x^{k}-$ степенной ряд с неотрицательными коэффичиентами и радиусом сходимости $R$. Предположим, что $0<R<\infty$, $\varphi(R)<\infty$, и пусть $R_{n}>0$ определяется равенством $\varphi_{n}\left(R_{n}\right)=\varphi(R)$, где $\varphi_{n}(x)=\sum_{k=0}^{n} c_{k} x^{k}, n \geqslant 1$. Тогда

$$
\lim _{n \rightarrow \infty} \varphi_{n}^{\prime}\left(R_{n}\right)=\varphi^{\prime}(R)
$$

где, как и раньше, $\varphi^{\prime}(R)$ понимается как сумма продифференцированного в точке $R$ ряда, определяющего $\varphi$.

ДокАЗАТЕльСтво. Вначале заметим, что $R_{n} \geqslant R$ и, значит, $\varphi_{n}^{\prime}\left(R_{n}\right) \geqslant \varphi_{n}^{\prime}(R)$, $n \geqslant 1$. Так же очевидно, что $\varphi_{n}^{\prime}(R)$ не убьвает по $n$ и $\lim _{n \rightarrow \infty} \varphi_{n}^{\prime}(R)=\varphi^{\prime}(R)$ (все эти утверждения вытекают из неотрицательности коэффициентов $\left.c_{k}\right)$. Поэтому

$$
\varliminf_{n \rightarrow \infty} \varphi_{n}^{\prime}\left(R_{n}\right) \geqslant \varphi^{\prime}(R)
$$

При $\varphi^{\prime}(R)=\infty$ это неравенство равносильно доказываемому утверждению.

В случае, когда $\varphi^{\prime}(R)<\infty$, воспользуемся легко проверяемьм фактом: если $\psi-$ многочлен степени $d$ с неотрицательными коэффициентами и $k \geqslant d, a \geqslant b \geqslant 0$, то $\psi(a) b^{k} \leqslant \psi(b) a^{k}$. Применив это неравенство к $\psi=\varphi^{\prime}, k=d=n-1, a=R_{n}, b=R$, получим

$$
\varphi_{n}^{\prime}\left(R_{n}\right) \leqslant \varphi_{n}^{\prime}(R)\left(1+R^{-1} \delta_{n}\right)^{n-1}
$$

где $\delta_{n}=R_{n}-R$.

Осталось убедиться, что второй множитель в правой части (6.14) стремится к единище при $n \rightarrow \infty$. 
По определению чисел $R_{n}, \delta_{n}$ и в силу монотонности функции $\varphi_{n}^{\prime}$

$$
\varphi(R)-\varphi_{n}(R)=\varphi_{n}\left(R+\delta_{n}\right)-\varphi_{n}(R) \geqslant \delta_{n} \varphi_{n}^{\prime}(R)
$$

Значит,

$$
\delta_{n} \leqslant \frac{\varphi(R)-\varphi_{n}(R)}{\varphi_{n}^{\prime}(R)} \leqslant c\left(\varphi(R)-\varphi_{n}(R)\right)
$$

где $c>0$ не зависит от $n$, если $n$ достаточно велико (так как $0<R<\infty$ и, следовательно, $\varphi^{\prime}(R)>0$, то можно положить $\left.c=2 / \varphi^{\prime}(R)\right)$.

$\mathrm{C}$ другой стороны,

$$
n\left(\varphi(R)-\varphi_{n}(R)\right)=\sum_{k=n+1}^{\infty} n c_{k} R^{k} \leqslant R \sum_{k=n+1}^{\infty} k c_{k} R^{k-1} .
$$

При $n \rightarrow \infty$ последнее выражение стремится к нулю, так как по условию $\varphi^{\prime}(R)<\infty$. Таким образом, $\varphi(R)-\varphi_{n}(R)=o(1 / n)$ и, значит, $\delta_{n}=o(1 / n)($ см. (6.15)). Но тогда $\left(1+R^{-1} \delta_{n}\right)^{n} \rightarrow 1$ при $n \rightarrow \infty$ и из (6.14) вытекает, что

$$
\varlimsup_{n \rightarrow \infty} \varphi_{n}^{\prime}\left(R_{n}\right) \leqslant \lim _{n \rightarrow \infty} \varphi_{n}^{\prime}(R)=\varphi^{\prime}(R)
$$

Вместе с (6.13) это дает (6.12). Лемма доказана.

6.7. ЗАмЕчАнИЕ. В дополнение к лемме 6.6 можно доказать, хотя нам это и не потребуется, что последовательность $\left\{\varphi_{n}^{\prime}\left(R_{n}\right)\right\}$ не убьвает; значит, при всех $n$ вьполняется неравенство $\varphi_{n}^{\prime}\left(R_{n}\right) \leqslant \varphi^{\prime}(R)$ и, тем самьм, $\varphi^{\prime}(R)=\sup _{n} \varphi^{\prime}\left(R_{n}\right)$. Если, однако, в формулировке леммы 6.6 возмутить частичную сумму $\varphi_{n}$, добавив к ней член того же ряда с достаточно большим номером, то ситуация, как мы сейчас увидим, резко изменится.

6.8. ЛЕммА. Пусть $\varphi(z)=\sum_{k=1}^{\infty} c_{k} z^{k}-$ бесконечный степенной ряд с неотрицательными коэффичиентами и радиусом сходимости $R$. Предположим, что $0<R<\infty, \varphi(R)<\infty$, и пусть при всех челых $n \geqslant 1$ и $m>n$ число $R_{n, m}$ определяется равенством $\varphi_{n, m}\left(R_{n, m}\right)=\varphi(R)$, где

$$
\varphi_{n, m}(z)=\varphi_{n}(z)+c_{m} z^{m}=\sum_{k=1}^{n} c_{k} z^{k}+c_{m} z^{m}
$$

Тогда существует такая последовательность номеров $\left\{n_{k}\right\}$, что

$$
\lim _{k \rightarrow \infty} \varphi_{n_{k}, n_{k+1}}^{\prime}\left(R_{n_{k}, n_{k+1}}\right)=\infty
$$


ДокАЗАТЕльство. Достаточно доказать, что при любом $n \geqslant 1$

$$
\varlimsup_{m \rightarrow \infty} \varphi_{n, m}^{\prime}\left(R_{n, m}\right)=\infty
$$

так как отсюда следует утверждение леммы.

По определению при $m>n$

$$
\begin{aligned}
\varphi_{n, m}^{\prime}\left(R_{n, m}\right) & =\varphi_{n}^{\prime}\left(R_{n, m}\right)+m c_{m} R_{n, m}^{m-1} \geqslant m c_{m} R_{n, m}^{m-1} \\
& =\frac{m}{R_{n, m}}\left(\varphi_{n, m}\left(R_{n, m}\right)-\varphi_{n}\left(R_{n, m}\right)\right) \\
& =\frac{m}{R_{n, m}}\left(\varphi(R)-\varphi_{n}\left(R_{n, m}\right)\right) .
\end{aligned}
$$

Очевидно, $R_{n, m} \geqslant R$ при всех $n$ и $m>n$. Покажем, что

$$
\varliminf_{m \rightarrow \infty} R_{n, m}=R, \quad n=1,2, \ldots
$$

Пусть при каком-то $n$ (6.18) не выполняется. Тогда при некотором $r>R$ и всех достаточно больших $m$ справедливо неравенство $R_{n, m} \geqslant r$, вследствие которого при тех же $r$ и $m$

$$
\varphi_{n, m}\left(R_{n, m}\right) \geqslant c_{m} R_{n, m}^{m} \geqslant c_{m} r^{m}
$$

Так как $r>R$, по формуле Коши-Адамара $\varlimsup_{m \rightarrow \infty} c_{m} r^{m}=\infty$ и в силу (6.19)

$$
\varlimsup_{m \rightarrow \infty} \varphi_{n, m}\left(R_{n, m}\right)=\infty
$$

что противоречит определению $R_{n, m}$. Из $(6.17),(6.18)$ получаем

$$
\varlimsup_{m \rightarrow \infty} \varphi_{n, m}^{\prime}\left(R_{n, m}\right) \geqslant \varliminf_{m \rightarrow \infty} \frac{m}{R_{n, m}}\left(\varphi(R)-\varphi_{n}\left(R_{n, m}\right)\right)=\infty,
$$

так как по условию $c_{k}>0$ при бесконечно многих $k$ и, значит, $\varphi(R)>\varphi_{n}(R)$ при всех $n$. Тем самым, вьполняется соотношение (6.16). Лемма доказана.

6.9. ПРЕДЛОЖЕНИЕ. Пусть $G$ - произвольный лепестковый граф и пусть функиия $f \in \mathscr{F}_{0}^{1}(G)$ такова, что матрица Больимана $A_{f}$ неустойчиво-положительна. Тогда найдутся две исчерпьвающие последовательности, $\left\{G_{k}^{1}\right\}$ и $\left\{G_{k}^{2}\right\}$, конечных подграфов графа $G$, для которых

$$
\lim _{k \rightarrow \infty} \mu^{f_{k}^{1}}=\mu^{f}, \quad \lim _{k \rightarrow \infty} \mu^{f_{k}^{2}}=\delta_{\infty},
$$

где $f_{k}^{i}=\left.f\right|_{X\left(G_{k}^{i}\right)}, \mu^{f_{k}^{i}}-f_{k}^{i}$-равновесная мера (определенная на $\bar{X}(G)$, но сосредоточенная на $\left.X\left(G_{k}^{i}\right)\right), i=1,2, \mu^{f}-f$-равновесная мера (определенная на $\bar{X}(G)$, но сосредоточенная на $X(G))$ и $\delta_{\infty}$ - вероятностная мера, сосредоточенная в точке $x^{\infty} \in \bar{X}(G)$ (см. предложсение 5.1). 
ДокАЗАТЕльство. Пусть $v$ - центральная вершина графа $G$ и $\mathscr{L}_{l}-$ множество лепестков длины $l$, входяших в $G$. Очевидно, $\mathscr{L}_{l}=\Gamma_{v v}^{0}(G, l)($ см. $\S 2)$ и, следовательно,

$$
Q_{v v}^{0}\left(A_{f}, l\right)=\sum_{\gamma \in \mathscr{L}_{l}} Q\left(A_{f}, \gamma\right), \quad l \geqslant 2 .
$$

Положим $c_{l}=Q_{v v}^{0}\left(A_{f}, l\right)$ и определим $\varphi(z), \varphi_{n}(z), \varphi_{n, m}(z), R, R_{n}, R_{n, m}$ так же, как это сделано в формулировках лемм 6.6 и 6.8. Поскольку матрища $A_{f}$ неустойчиво-положительна, условия обеих этих лемм вьполняются. Пусть $\left\{n_{k}\right\}-$ последовательность, сушествование которой гарантируется леммой 6.8. Имея в виду, что множество $\mathscr{L}_{l}$ при некоторых или даже при всех $l$ может быть бесконечным, возьмем числовую последовательность $\left\{\varepsilon_{n}\right\}, \varepsilon_{n}>0$, монотонно сходящуюся к нулю, выбор которой в дальнейшем будет уточнен, и подберем при каждом $l \geqslant 2$ такую последовательность конечных множеств $\mathscr{L}_{l, n} \subset \mathscr{L}_{l}, n=1,2, \ldots$, что

$$
\mathscr{L}_{l, n} \subseteq \mathscr{L}_{l, n+1}, \quad \sum_{\gamma \in \mathscr{L}_{l, n}} Q\left(A_{f}, \gamma\right) \geqslant\left(1-\varepsilon_{n}\right) c_{l}, \quad n=2,3, \ldots,
$$

$\left(\right.$ если $\mathscr{L}_{l}=\varnothing$, то и $\mathscr{L}_{l, n}=\varnothing$; в этом случае суммы, стояшие в $(6.21)$ и $(6.22)$, считаются равньми нулю).

В качестве $G_{k}^{1}, G_{k}^{2}$ возьмем главные подграфы граффа $G$, образованные всеми лепестками, входящими соответственно в $\bigcup_{l=2}^{k} \mathscr{L}_{l, k}$ и в $\left(\bigcup_{l=2}^{n_{k}} \mathscr{L}_{l, n_{k}}\right) \cup \mathscr{L}_{n_{k+1}, n_{k}}$. Из (6.22) вытекает, что $\left\{G_{k}^{1}\right\}$ и $\left\{G_{k}^{2}\right\}$ - исчерпьваюшие последовательности конечных подграфов графа $G$. Докажем, что если $\varepsilon_{n}$ стремится к нулю достаточно быстро, то вьполняются соотношения (6.20). Начнем с первого из них.

Положим для краткости

$$
\begin{gathered}
a_{l, k}=Q_{v v}^{0}\left(A_{f_{k}^{1}}, l\right), \quad \psi_{k}(z)=\varphi_{v v}^{0}\left(A_{f_{k}^{1}}, z\right), \quad \tilde{\psi}_{k}(z)=\varphi_{v v}^{0}\left(A_{f_{k}^{2}}, z\right), \\
r_{k}=R\left(A_{f_{k}^{1}}\right), \quad \tilde{r}_{k}=R\left(A_{f_{k}^{2}}\right), \quad k, l \geqslant 2 .
\end{gathered}
$$

По построению

$$
\begin{gathered}
a_{l, k}=\sum_{\gamma \in \mathscr{L}_{l, k}} Q\left(A_{f}, \gamma\right), \quad \psi_{k}(z)=\sum_{l=2}^{k} a_{l, k} z^{k}, \\
\tilde{\psi}_{k}(z)=\psi_{n_{k}}(z)+a_{n_{k+1}, n_{k}} z^{n_{k+1}}, \quad k, l \geqslant 2 .
\end{gathered}
$$

Следовательно (см. (6.22)),

$$
\begin{gathered}
\left(1-\varepsilon_{k}\right) c_{l} \leqslant a_{l, k} \leqslant c_{l}, \quad k \geqslant 2, l \leqslant k ; \quad r_{k} \geqslant R_{k}, \tilde{r}_{k} \geqslant R_{n_{k}, n_{k+1}}, \\
\left(1-\varepsilon_{k}\right) \varphi_{k}(r) \leqslant \psi_{k}(r) \leqslant \varphi_{k}(r), \quad\left(1-\varepsilon_{k}\right) \varphi_{k}^{\prime}(r) \leqslant \psi_{k}^{\prime}(r) \leqslant \varphi_{k}^{\prime}(r), \quad r \geqslant 0 .
\end{gathered}
$$

Пользуясь этим, оценим разность $r_{k}-R_{k}$. Так как $\varphi_{k}\left(R_{k}\right)=\psi_{k}\left(r_{k}\right)$ и $\psi_{k}^{\prime}$ монотонно возрастает, мы имеем

$$
0 \leqslant r_{k}-R_{k} \leqslant \frac{\psi_{k}\left(r_{k}\right)-\psi_{k}\left(R_{k}\right)}{\psi_{k}^{\prime}\left(R_{k}\right)}=\frac{\varphi_{k}\left(R_{k}\right)-\psi_{k}\left(R_{k}\right)}{\psi_{k}^{\prime}\left(R_{k}\right)} \leqslant \frac{\varepsilon_{k}}{\left(1-\varepsilon_{k}\right) \varphi_{k}^{\prime}\left(R_{k}\right)} .
$$


Отсюда следует, что при достаточно больших $k$

$$
0 \leqslant r_{k}-R_{k} \leqslant \beta \varepsilon_{k}
$$

где $\beta=2 / \varphi^{\prime}(R)$ (см. лемму 6.6). Если вьполняется последнее неравенство и, кроме того, $r_{k} \leqslant 2 R_{k}$ (т.е. $\left.\varepsilon_{k} \leqslant R_{k} / \beta\right)$, то

$$
\begin{aligned}
\left|\varphi_{k}^{\prime}\left(R_{k}\right)-\psi_{k}^{\prime}\left(r_{k}\right)\right| & =\left|\sum_{l=2}^{k} l\left(c_{l} R_{k}^{l-1}-a_{l, k} r_{k}^{l-1}\right)\right| \\
& \leqslant \sum_{l=2}^{k} l\left|\left(c_{l}-a_{l, k}\right) R_{k}^{l-1}+\left(R_{k}^{l-1}-r_{k}^{l-1}\right) a_{l, k}\right| \\
& \leqslant \sum_{l=2}^{k} l\left|c_{l}-a_{l, k}\right| R_{k}^{l-1}+\sum_{l=2}^{k} l\left|R_{k}^{l-1}-r_{k}^{l-1}\right| c_{l} \\
& \leqslant \varepsilon_{k} \sum_{l=2}^{k} l c_{l} R_{k}^{l-1}+\sum_{l=2}^{k} l c_{l}\left(r_{k}-R_{k}\right)(l-1) r_{k}^{l-2} \\
& \leqslant \varepsilon_{k}(1+\beta)\left[\varphi_{k}^{\prime}\left(R_{k}\right)+\sum_{l=2}^{k} l(l-1) 2^{l-2} c_{l} R_{k}^{l-2}\right] .
\end{aligned}
$$

Поэтому последовательность $\left\{\varepsilon_{k}\right\}$ можно выбрать так, что $\left|\varphi_{k}^{\prime}\left(R_{k}\right)-\psi_{k}^{\prime}\left(r_{k}\right)\right| \rightarrow 0$ при $k \rightarrow \infty$. По лемме $6.6 \lim _{k \rightarrow \infty} \varphi_{k}^{\prime}\left(R_{k}\right)=\varphi^{\prime}(R)$. Значит, тот же предел имеет и $\psi_{k}^{\prime}\left(r_{k}\right)$. Так как $\psi_{k}^{\prime}\left(r_{k}\right)=D_{v}\left(A_{f_{k}^{1}}\right)($ см. $(6.23)$ и $(4.7))$, отсюда в силу теоремы 6.4 вытекает первое из соотношений (6.22).

Второе доказывается еще проще. Действительно, в силу (6.23), (6.24)

$$
\tilde{\psi}_{k}^{\prime}\left(\tilde{r}_{k}\right) \geqslant\left(1-\varepsilon_{n_{k}}\right) \varphi_{n_{k}, n_{k+1}}^{\prime}\left(\tilde{r}_{k}\right) \geqslant\left(1-\varepsilon_{n_{k}}\right) \varphi_{n_{k}, n_{k+1}}^{\prime}\left(R_{n_{k}, n_{k+1}}\right),
$$

а так как последовательность $\left\{n_{k}\right\}$ удовлетворяет условию

$$
\lim _{k \rightarrow \infty} \varphi_{n_{k}, n_{k+1}}^{\prime}\left(R_{n_{k}, n_{k+1}}\right)=\infty
$$

(см. лемму 6.8), достаточно еще раз применить теорему 6.4. Предложение доказано.

В случае, когда $f \equiv 0$, предложение 6.9 можно значительно усилить. Однако прежде чем сделать это, убедимся в том, что существуют лепестковые графы $G$ с неустойчиво-положительной матрицей смежности. Очевидно, это свойство, выраженное в терминах распределения $\left\{N_{l}(G), l \geqslant 2\right\}$, состоит в том, что степенной ряд $\sum_{l} N_{l}(G) z^{l}=\varphi(z)$ имеет ненулевой радиус сходимости $R$, причем $\varphi(R)=1, \varphi^{\prime}(R)$ $<\infty$.

Возьмем любое натуральное $m>2$, для которого $\sum_{l=m+1}^{\infty} 2^{-l}\left[2^{l} l^{-3}\right]=s<1$ (здесь $[\cdot]$ обозначает целую часть числа). Тогда $1-s=\sum_{l=1}^{\infty} \varepsilon_{l} 2^{-l}$, где $\varepsilon_{l} \in\{0,1\}$, и достаточно положить $N_{2}(G)=2 \varepsilon_{1}+\varepsilon_{2}, N_{l}(G)=\varepsilon_{l}$ при $3 \leqslant l \leqslant m$ и $N_{l}(G)=$ $\left[2^{l} l^{-3}\right]+\varepsilon_{l}$ при $l \geqslant m+1$ (очевидно, при этом будет $R(G)=1 / 2$ ).

До конца этого параграффа мы будем рассматривать $f$-равновесные меры на пространствах $X(G)$ при различных $G$, предполагая каждый раз, что $f=f_{0} \equiv 0$. В этом случае все интересуюшие нас объекты определяются графом $G$. Будем поэтому писать $A_{G}$ и $\mu^{G}$ вместо $A_{f}$ и $\mu^{f}$ соответственно (заметим, что $A_{G}$ - матрица смежности граф̆а $G)$. 
6.10. ПРЕДЛОЖЕНИЕ. Пусть $G$ - лепестковый граф с неустойчиво-положительной матрищей $A_{G}$. Тогда для всякого замкнутого множества $\mathscr{M} \subset\left\{c \mu_{G}+(1-c) \delta_{\infty}\right.$, $0 \leqslant c \leqslant 1\}$ найдется такая исчерпшвающая последовательность конечных подграфов $G_{n} \subset G$, что множество предельных точек последовательности мер $\mu^{G_{n}}$ совпадает $c \mathscr{M}$.

ДокаЗАТЕльство. Пусть $v$ - центральная вершина графа $G$. В силу теоремы 6.4 достаточно найти для любого замкнутого подмножества $\mathscr{D}$ расширенной полупрямой $\left[D_{v}\left(A_{G}\right),+\infty\right]$ такую исчерпьвающую последовательность подграфов $G_{n} \subset G$, что множество предельных точек числовой последовательности $\left\{D_{v}\left(A_{G_{n}}\right)\right\}$ есть $\mathscr{D}$. Эта цель будет достигнута, если для любой последовательности чисел $\alpha_{n}>D_{v}\left(A_{G}\right)$ мы определим $\left\{G_{n}\right\}$ так, что при всех достаточно больших $n$ будет выполняться неравенство

$$
\left|D_{v}\left(A_{G_{n}}\right)-\alpha_{n}\right|<\frac{1}{n}
$$

Будем строить графы $G_{n}$ по индукции. В качестве $G_{1}$ возьмем главньй подграф графа $G$, образованньй любьми двумя лепестками разной длины. Предположим, что $G_{1}, \ldots, G_{k}(k \geqslant 1)$ уже построены. Пусть $k^{*}>k$ и $G\left(k^{*}\right)$ - главный подграф графа $G$, образованньй всеми лепестками длины $\leqslant k^{*}$ (поскольку из допустимости $A_{G}$ следует, что $N_{l}(G)<\infty$ при всех $l \geqslant 2$, этот подграфф конечен). Положив

$$
\psi(z)=\varphi_{v v}^{0}\left(A_{G\left(k^{*}\right)}, z\right), \quad R=R\left(A_{G}\right), \quad R\left(k^{*}\right)=R\left(A_{G\left(k^{*}\right)}\right),
$$

подберем настолько большое $k^{*}$, что $G_{k} \subset G\left(k^{*}\right)$ и

$$
\left|\psi^{\prime}(R)-D_{v}\left(A_{G}\right)\right|<\frac{1}{4(k+1)} .
$$

С учетом леммы 6.6 можно также считать (быть может, еше увеличив $k^{*}$ ), что

$$
\left|\psi^{\prime}\left(R\left(k^{*}\right)\right)-D_{v}\left(A_{G}\right)\right|<\frac{1}{4(k+1)}
$$

Очевидно, $R<R\left(k^{*}\right)<1$ (это вытекает из наличия в $G\left(k^{*}\right)$ лепестков разной длины и целочисленности коэффициентов $\left.Q_{v v}\left(A_{G\left(k^{*}\right)}, n\right)\right)$.

Граф $G_{k+1}$ будет получаться из $G\left(k^{*}\right)$ добавлением некоторого числа $b \leqslant N_{m}(G)$ лепестков длины $m>k^{*}$, где $m$ и $b$ еще подлежат определению.

Положим

$$
\psi_{m, b}(z)=\psi(z)+b z^{m}, \quad d_{m, b}=\psi_{m, b}^{\prime}\left(R_{m, b}\right), \quad m \geqslant k^{*}+1, \quad 0 \leqslant b \leqslant N_{m},
$$

где $N_{m}=N_{m}(G)$ и $R_{m, b}>0$ определяется соотношением $\psi_{m, b}\left(R_{m, b}\right)=1$. Из монотонности $\psi$ вытекает, что $R<R_{m, b+1} \leqslant R_{m, b} \leqslant R\left(k^{*}\right)$ и

$$
\begin{aligned}
d_{m, b+1}-d_{m, b} & =\psi^{\prime}\left(R_{m, b+1}\right)-\psi^{\prime}\left(R_{m, b}\right)+m b\left(R_{m, b+1}^{m-1}-R_{m, b}^{m-1}\right)+m R_{m, b+1}^{m-1} \\
& \leqslant \psi^{\prime}\left(R\left(k^{*}\right)\right)-\psi^{\prime}(R)+\left(R\left(k^{*}\right)\right)^{m-1} m .
\end{aligned}
$$


При доказательстве леммы 6.8 мы установили (см. (6.18)), что выбором подходящего $m$ можно сделать производную $\psi_{m, N_{m}}^{\prime}\left(R_{m, N_{m}}\right)$ как угодно большой. С учетом этого факта, а также неравенства $R\left(k^{*}\right)<1$ подберем $m=m^{*}$, удовлетворяюшее условиям

$$
\left(R\left(k^{*}\right)\right)^{m^{*}-1} m^{*} \leqslant \frac{1}{2(k+1)}, \quad \psi_{m^{*}, N_{m^{*}}}^{\prime}\left(R_{m^{*}, N_{m^{*}}}\right)>\alpha_{k+1} .
$$

Применив $(6.26),(6.27)$ и первое из этих условий к $(6.28)$, получим

$$
d_{m^{*}, b+1}-d_{m^{*}, b} \leqslant \frac{1}{k+1} .
$$

Отсюда с помощью второго из условий (6.29) можно заключить, что сушествует $b=b^{*} \leqslant N_{m^{*}}$, для которого

$$
\left|d_{m^{*}, b^{*}}-\alpha_{k+1}\right| \leqslant \frac{1}{k+1}
$$

Определив граф $G_{k+1}$ указанным вьше способом при $m=m^{*}, b=b^{*}$, непосредственно убеждаемся, что $D_{v}\left(A_{G_{k+1}}\right)=d_{m^{*}, b^{*}}$ и, значит, (6.25) вьполняется также при $n=k+1$. Предложение доказано.

\section{$\S$ 7. Асимптотика дискретных инвариантных мер}

Очевидно, всякая дискретная $S$-инвариантная мера, определенная на $X(G)$, сосредоточена на множестве периодических точек преобразования $S$ и принимает одинаковые значения на всех одноточечных множествах, входяших в одну периодическую орбиту. Мы будем рассматривать дискретные инвариантные меры, сосредоточенные на периодических орбитах фиксированного периода и определяемые функциями $f: X(G) \rightarrow \mathbb{R}$. Пусть, как и раньше, $\operatorname{Fix}_{n}(G)=\left\{x \in X(G): S^{n} x=x\right\}, n=1,2, \ldots$ При $B \in \mathscr{B}$, где $\mathscr{B}=\mathscr{B}(X(G))$ - совокупность борелевских подмножеств пространства $X(G)$, положим

$$
Q_{B}(G, f, n)=\sum_{x \in B \cap \mathrm{Fix}_{n}(G)} \exp \sum_{i=0}^{n-1} f\left(S^{i} x\right)
$$

если $\operatorname{Fix}_{n}(G) \neq \varnothing$, и $Q_{B}(G, f, n)=0$ в противном случае (cp. (2.1)). Если $Y \subset X(G)$, $Y \cap \operatorname{Fix}_{n}(G) \neq \varnothing$ и $Q_{Y}(G, f, n)<\infty$, то равенство

$$
\mu_{n}^{f, Y}(B)=\frac{Q_{B \cap Y}(G, f, n)}{Q_{Y}(G, f, n)}, \quad B \in \mathscr{B},
$$

определяет на $X(G)$ дискретную вероятностную меру, которая, как легко проверить, будет $S$-инвариантной, если $S$-инвариантно множество $Y$. Возможен случай, когда для некоторых $Y \subset X(G)$ мера $\mu_{n}^{f, Y}$ определена, а мера $\mu_{n}^{f, X(G)}$-нет; если же обе они существуют, то $\mu_{n}^{f, Y}$ есть, очевидно, условная мера при условии $Y$, отвечающая мере $\mu_{n}^{f, X(G)}$. 
Ниже нам потребуется несколько вспомогательных утверждений. Первое из них, неравенство Рюэля [43], справедливо в весьма обшей ситуашии.

7.1. Лемма. Пусть $(X, \mathscr{B})$ - измеримое пространство, $S: X \rightarrow X-$ измеримое отображсние $и f: X \rightarrow \mathbb{R}, g: X \rightarrow \mathbb{R}$ - измеримые функции, причем $g$ ограничена. Предположсим, что для $n \in \mathbb{N}$ и $S$-инвариантного множсества $Y \in \mathscr{B}$ формулами, аналогичными (7.1) и (7.2) (в которьх надо опустить символ $G$ ), определень константы $Q_{Y}(f, n)$ и мера $\mu_{n}^{f, Y}$. Тогда

$$
n^{-1} \ln Q_{Y}(f+g, n) \geqslant n^{-1} \ln Q_{Y}(f, n)+\int g d \mu_{n}^{f, Y}
$$

Хотя предположения, при которых неравенство (7.3) получено в [43], существенно отличаются от наших, приведенное там доказательство опирается лишш на сходимость ряда, определяюшего $Q_{Y}(f, n$,$) , и ограниченность функции g$.

Возвращаясь к СЦМ $(X(G), S)$, заметим, что $\operatorname{Fix}_{n}(G) \neq \varnothing$ при всех достаточно больших $n$ вида $k d$, где $d=d(G)$ - период граффа $G$, и $\operatorname{Fix}_{n}(G)=\varnothing$, если $n \neq k d$. С учетом этого положим для $B \in \mathscr{B}$

$$
\begin{aligned}
& q_{B}^{-}(f)=\varliminf_{k \rightarrow \infty}(k d)^{-1} \ln Q_{B}(G, f, k d), \\
& q_{B}^{+}(f)=\varlimsup_{k \rightarrow \infty}(k d)^{-1} \ln Q_{B}(G, f, k d) .
\end{aligned}
$$

7.2. ЗАмечАниЕ. Если $v \in V(G), B=C(v)$, то при каждом $n$ множество $B \cap$ $\operatorname{Fix}_{n}(G)$ находится во взаимнооднозначном соответствии с множеством $\Gamma_{v v}(G, n)$, а если к тому же $f \in \mathscr{F}_{0}^{1}(G)$, то $Q_{B}(G, f, n)=Q_{v v}\left(A_{f}, n\right)$. Следовательно (см. теорему 4.4 и замечание 2.2 ),

$$
q_{B}^{-}(f)=q_{B}^{+}(f)=-\ln R\left(A_{f}\right)=P^{\text {int }}(X(G), S, f)
$$

и положительность радиуса сходимости $R\left(A_{f}\right)$ гарантирует существование меры $\mu_{n d}^{f, B}$, если $n$ достаточно велико.

7.3. Лемма. Пусть $f \in \mathscr{F}_{0}^{1}(G), \bar{g} \in C(\bar{X}(G)), g=\left.\bar{g}\right|_{X(G)} u v \in V(G)$. Тогда

$$
q_{C(v)}^{-}(f+g)=q_{C(v)}^{+}(f+g)=P^{\mathrm{int}}(X(G), S, f+g) .
$$

ДокАЗАТЕльство. Из (7.1), (7.4) непосредственно вытекает, что величины $q_{C(v)}^{-}(f+g)$ и $q_{C(v)}^{+}(f+g)$ при фиксированных $f$ и $v$ непрерывно зависят от $\bar{g}$ как элемента пространства $C(\bar{X}(G))$ и не меняются при замене $\bar{g}$ на $S^{*} \bar{g}$. То же самое верно и для $P^{\mathrm{int}}(X(G), S, f+g)$, так как $P^{\mathrm{int}}(X(G), S, f+g)=P_{f}(\bar{g})$ (см. (5.1) и лемму 5.2). Поэтому достаточно доказать (7.5) для функций $\bar{g}$, удовлетворяюших условию $g \in \bigcup_{k \geqslant 2} \mathscr{F}_{0}^{k}(G)$.

Пусть $g \in \mathscr{F}_{0}^{k}(G)$, где $k \geqslant 1$ таково, что $C(v) \cap \operatorname{Fix}_{k}(G) \neq \varnothing$ (ясно, что это не ограничивает обшности). Тогда существует непустой сплошной $v$-цилиндр $C \subset X(G)$ с 
носителем $\{0, \ldots, k\}$ (см. определение 5.5). Покажем, что для любого такого цилиндpa

$$
q_{C}^{-}(f+g)=q_{C(v)}^{-}(f+g), \quad q_{C}^{+}(f+g)=q_{C(v)}^{+}(f+g) .
$$

Очевидно, при некотором $\gamma \in \Gamma_{v v}(G, k)$

$$
C=C(\gamma)=\left\{x \in X(G):\left(x_{0}, \ldots, x_{k}\right)=\gamma\right\}
$$

и так как $C \subset C(v)$, из (7.1) вытекает, что

$$
Q_{C}(G, f+g, n) \leqslant Q_{C(v)}(G, f+g, n), \quad n=1,2, \ldots
$$

Пусть $y=\left(y_{i}, i \in \mathbb{Z}\right)$ - произвольная точка из $C(v) \cap \operatorname{Fix}_{n}(G), n \geqslant k$, и $\gamma_{y}=$ $\left(y_{0}, \ldots, y_{n}\right)$. В таком случае $y_{0}=y_{n}=v$ и $\left(y_{m n}, y_{m n+1}, \ldots, y_{(m+1) n}\right)=\gamma_{y}$ при всех $m \in \mathbb{Z}$. Рассмотрим последовательность $\hat{y}=\left(\hat{y}_{i}, i \in \mathbb{Z}\right)$, определяемую условием

$$
\left(\hat{y}_{m(n+k)}, \hat{y}_{m(n+k)+1}, \ldots, \hat{y}_{(m+1)(n+k)}\right)=\gamma \gamma_{y}, \quad m \in \mathbb{Z} .
$$

Из предположений $f \in \mathscr{F}_{0}^{1}(G)$ и $g \in \mathscr{F}_{0}^{k}(G)$ следует, что при $n>k$

$$
\begin{aligned}
& \exp \sum_{i=0}^{n+k-1}\left[f\left(S^{i} \hat{y}\right)+g\left(S^{i} \hat{y}\right)\right] \\
&=\exp \left\{\sum_{i=0}^{k-1} f\left(S^{i} \hat{y}\right)+\sum_{i=0}^{k-1} g\left(S^{i} \hat{y}\right)\right. \\
&\left.\quad+\sum_{i=0}^{n-1}\left[f\left(S^{i} y\right)+g\left(S^{i} y\right)\right]+\sum_{i=k}^{n+k-1} g\left(S^{i} \hat{y}\right)-\sum_{i=0}^{n-1} g\left(S^{i} y\right)\right\} \\
&=\exp \left\{\sum_{i=0}^{k-1} f\left(S^{i} \hat{y}\right)+\sum_{i=0}^{k-1} g\left(S^{i} \hat{y}\right)\right. \\
&\left.\quad+\sum_{i=0}^{n-1}\left[f\left(S^{i} y\right)+g\left(S^{i} y\right)\right]+\sum_{i=n+1}^{n+k-1} g\left(S^{i} \hat{y}\right)-\sum_{i=n+1-k}^{n-1} g\left(S^{i} \hat{y}\right)\right\} \\
& \geqslant Q\left(A_{f}, \gamma\right) \exp (-3 k\|\bar{g}\|) \exp \sum_{i=0}^{n-1}\left[f\left(S^{i} y\right)+g\left(S^{i} y\right)\right]
\end{aligned}
$$

а так как, очевидно, $\hat{y} \in C \cap \operatorname{Fix}_{n+k}(S)$ и разным $y$ отвечают разные $\hat{y}$, мы получаем

$$
Q_{C}(G, f+g, n+k) \geqslant \beta Q_{C(v)}(G, f+g, n), \quad n \geqslant k,
$$

где $\beta$ не зависит от $n$. Это неравенство в сочетании с (7.7) приводит к (6.6), так как $k$ кратно $d$. Поэтому из $(7.7),(7.8)$ вытекает (7.6). 
Для завершения доказательства леммы воспользуемся преобразованием $\Phi_{k+1}$, переводяшим граф $G$, пространство $X(G)$ и функции $f, g$ соответственно в $G_{k+1}$, $X\left(G_{k+1}\right), f_{k+1}$ и $g_{k+1}$ (см. $\left.\S 1\right)$. Из определения следует, что

$$
f_{k+1}, g_{k+1} \in \mathscr{F}_{0}^{0}\left(G_{k+1}\right), \quad \Phi_{k+1} \operatorname{Fix}_{n}(G)=\operatorname{Fix}_{n}\left(G_{k+1}\right)
$$

и $\Phi_{k+1} C$ - цилиндр в $X\left(G_{k+1}\right)$ с носителем $\{0\}$. Тогда согласно замечанию 7.2

$$
q_{\Phi_{k+1} C}^{-}\left(f_{k+1}+g_{k+1}\right)=q_{\Phi_{k+1} C}^{+}\left(f_{k+1}+g_{k+1}\right)=P^{\text {int }}\left(X\left(G_{k+1}\right), S, f_{k+1}+g_{k+1}\right) .
$$

С другой стороны,

$$
\begin{aligned}
q_{\Phi_{k+1} C}^{-}\left(f_{k+1}+g_{k+1}\right) & =q_{C}^{-}(f+g), \\
q_{\Phi_{k+1} C}^{+}\left(f_{k+1}+g_{k+1}\right) & =q_{C}^{+}(f+g), \\
P^{\operatorname{int}}\left(X\left(G_{k+1}\right), S, f_{k+1}+g_{k+1}\right) & =P^{\text {int }}(X(G), S, f+g) ;
\end{aligned}
$$

первые два соотношения непосредственно следуют из определения, а последнее - из того факта, что $\Phi_{k+1}$ - гомеоморфизм. Теперь (7.5) вытекает из (7.6). Лемма доказана.

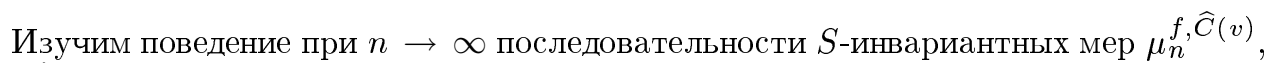
где $\widehat{C}(v)=\bigcup_{n \in \mathbb{Z}} S^{n} C(v)$ - наименьшее $S$-инвариантное множество, содержащее цилиндр $C(v)$. Здесь мы неоднократно будем пользоваться тем, что для любой функции $h: X(G) \rightarrow \mathbb{R}$

$$
Q_{C(v)}(G, h, n) \leqslant Q_{\widehat{C}(v)}(G, h, n) \leqslant n Q_{C(v)}(G, h, n), \quad n \geqslant 1
$$

(это непосредственно вытекает из определения), и, следовательно,

$$
q_{C(v)}^{-}(h)=q_{\widehat{C}(v)}^{-}(h), \quad q_{C(v)}^{+}(h)=q_{\widehat{C}(v)}^{+}(h) .
$$

Как и в $\S 6$, будем считать все рассматриваемые меры определенными на $\bar{X}(G)$, а их сходимость понимать как слабую сходимость на этом пространстве. Сейчас мы снова воспользуемся субдифференциалом $\partial P_{f}(g)$, введенньм в $\S 6$.

7.4. ПРЕДЛОЖЕНИЕ. Если $f \in \mathscr{F}_{0}^{1}(G), R\left(A_{f}\right)>0, v \in V(G) u \mu$ - произвольная предельная точка последовательности мер $\mu_{n d}^{f, \widehat{C}(v)}$, әде $d=d(G)$, то $\mu \in \partial P_{f}(0)$.

ДокАЗАТЕльСтво. Вначале заметим, что так как $R\left(A_{f}\right)>0$, при всех достаточно больших $n$ выполняются неравенства $0<Q_{\widehat{C}(v)}(G, f, n d)<\infty$ (см. (7.8) и замечание 7.2). Следовательно, при таких $n$ определена мера $\mu_{n d}^{f, \widehat{C}(v)}$. Пусть

$$
\mu=\lim _{k \rightarrow \infty} \mu_{n_{k} d}^{f, \widehat{C}(v)}, \quad \bar{g} \in C(\bar{X}(G)), \quad g=\left.\bar{g}\right|_{\bar{X}(G)} .
$$


Положив в (7.3) $Y=\widehat{C}(v), n=n_{k}$ и перейдя к пределу при $k \rightarrow \infty$, получим

$$
\begin{aligned}
\varlimsup_{k \rightarrow \infty}\left(n_{k} d\right)^{-1} \ln Q_{\widehat{C}(v)}\left(G, f+g, n_{k} d\right) & \\
& \geqslant \underset{k \rightarrow \infty}{\frac{\lim }{\rightarrow \rightarrow \infty}}\left(n_{k} d\right)^{-1} \ln Q_{\widehat{C}(v)}\left(G, f, n_{k} d\right)+\int_{\bar{X}(G)} \bar{g} d \mu .
\end{aligned}
$$

С учетом (7.9) можно заменить в (7.10) $\widehat{C}(v)$ на $C(v)$, а затем, пользуясь леммой 7.3 , получить неравенство

$$
P^{\mathrm{int}}(X(G), S, f+g) \geqslant P^{\mathrm{int}}(X(G), S, f)+\int \bar{g} d \mu .
$$

Условие $R\left(A_{f}\right)>0$ гарантирует, что обе его части конечны (см. замечание 7.2). Но тогда $\mu \in \partial P_{f}(0)$ (см. (5.1) и определение 5.3), что и требовалось доказать.

Комбинируя предложение 7.4 с теоремой 5.7, моментально получаем следуюший результат.

7.5. СлЕДСТВИЕ. Пусть $f \in \mathscr{F}_{0}^{1}(G), v \in V(G) u d=d(G)$. Предположим, что $R\left(A_{f}\right)>0$. Тогда:

1) если матрица $A_{f}$ невозвратна или нуль-возвратна, то $\lim _{n \rightarrow \infty} \mu_{n d}^{f, \widehat{C}(v)}$ $=\delta_{\infty}$

2) если матрица $A_{f}$ устойчиво-положительна, то $\lim _{n \rightarrow \infty} \mu_{n d}^{f, \widehat{C}(v)}=\mu^{f}$.

В следствии 7.5 не упоминается случай неустойчиво-положительной матрицы $A_{f}$, так как для его изучения методы настояшей главы недостаточны. Мы рассмотрим этот случай в гл. 3 , посвященной динамическим дзета-ффункциям.

Перейдем к изучению мер $\mu_{n}^{f, X(G)}$, для которых мы в дальнейшем будем употреблять сокращенное обозначение $\mu_{n}^{f}$.

7.6. Teopema. Пусть $f \in \mathscr{F}_{0}^{1}(G), d=d(G), R\left(A_{f}\right)>0 u Q_{X(G)}(G, f, n d)<\infty$ при всех достаточно больших $n$. Предположим, что матрича $A_{f}$ невозвратна или нуль-возвратна. Тогда

$$
\lim _{n \rightarrow \infty} \mu_{n d}^{f}=\delta_{\infty}
$$

ДокАЗАТЕльство. Как уже отмечалось, множество $\operatorname{Fix}_{n d}(G)$ непусто при всех достаточно больших $n$. Поэтому условие $Q_{X(G)}(G, f, n d)<\infty$ гарантирует сушествование при этих $n$ меры $\mu_{n d}^{f}$. Из (7.1) и определения множества $\widehat{C}(v), v \in V(G)$, видно, что при всех $v \in V(G)$

$$
\begin{gathered}
Q_{\widehat{C}(v) \cap C(v)}(G, f, n d)=Q_{C(v)}(G, f, n d), \\
Q_{\widehat{C}(v)}(G, f, n d) \leqslant Q_{X(G)}(G, f, n d),
\end{gathered}
$$

а потому

$$
\mu_{n d}^{f}(C(v)) \leqslant \mu_{n d}^{f, \widehat{C}(v)}(C(v))
$$

и если матрица $A_{f}$ невозвратна или нуль-возвратна, то (7.11) вытекает из п. 1 следствия 7.5. Теорема доказана.

В случае, когда матрица $A_{f}$ положительно-возвратна, мы вынуждены пока ограничиться описанием возможных предельных точек последовательности $\left\{\mu_{n d}^{f}\right\}$. Начнем с известной тауберовой теоремы Эрдеша-Феллера-Полларда [48]. 
7.7. Лемма. Пусть $\varphi(z)$ и $r$ - соответственно сумма и радиус сходимости степенного ряда с неотрицательными коэффициентами $a_{n}, n \geqslant 1$, и пусть $R \in(0, r]$ таково, что $\varphi(R)=1$. Тогда для коэффициентов $b_{n}$ разложсения в степенной ряд функции $1 /(1-\varphi)$ справедливо соотношение

$$
\lim _{n \rightarrow \infty} b_{n d} R^{n d}=\frac{d}{R \varphi^{\prime}(R)}
$$

əде $d=\mathrm{HОД}\left\{n: a_{n}>0\right\}$ u $\varphi^{\prime}(R)$ при $R=r$ понимается как сумма ряда $\sum_{n=1}^{\infty} n a_{n} r^{n-1}$ (конечная или бесконечная).

Следующая теорема до некоторой степени аналогична теореме 6.4.

7.8. ТЕОремА. Пусть выполнены условия теоремы 7.6. Предположим, что матрица $A_{f}$ положительно-возвратна. Тогда для сходимости последовательности мер $\mu_{n_{k} d}^{f}$, где $n_{k} \rightarrow \infty$ при $k \rightarrow \infty$, необходимо и достаточно, чтобь существовал предел

$$
u\left(f,\left\{n_{k}\right\}\right)=\lim _{k \rightarrow \infty} Q_{X(G)}\left(G, f, n_{k} d\right) R^{n_{k} d},
$$

где $R=R\left(A_{f}\right)$. Если это условие выполнено, то

$$
\lim _{k \rightarrow \infty} \mu_{n_{k} d}^{f}=\alpha \mu^{f}+(1-\alpha) \delta_{\infty}
$$

где

$$
\alpha=\frac{d}{u\left(f,\left\{n_{k}\right\}\right)}
$$

ДокАЗАТЕЛЬСТВо. Согласно замечанию 7.2

$$
Q_{C(v)}(G, f, n)=Q_{v v}\left(A_{f}, n\right), \quad n \geqslant 1, \quad v \in V(G) .
$$

Значит (см. §2),

$$
\sum_{n=1}^{\infty} Q_{C(v)}(G, f, n) z^{n}=\varphi_{v v}\left(A_{f}, z\right)=\frac{1}{1-\varphi_{v v}^{0}\left(A_{f}, z\right)}-1 .
$$

Применив к функции $\varphi(z)=\varphi_{v v}\left(A_{f}, z\right)$ лемму 7.7, получим $($ см. $(4.7),(4.12))$

$$
\lim _{n \rightarrow \infty} Q_{C(v)}(G, f, n d) R^{n d}=\frac{d}{R D_{v}\left(A_{f}\right)}=d \mu^{f}(C(v)), \quad v \in V(G) .
$$

Так как по определению (см. (7.2))

$$
\mu_{n_{k} d}^{f}(\bar{C})=\mu_{n_{k} d}^{f}(C)=\frac{Q_{C(v)}\left(G, f, n_{k} d\right) R^{n_{k} d}}{Q_{X(G)}\left(G, f, n_{k} d\right) R^{n_{k} d}},
$$


а множество $\bar{C}(v) \subset \bar{X}(G)$ не только замкнуто, но и открыто, из (7.15) следует, что сушествование предела $\lim _{k \rightarrow \infty} Q_{X(G)}\left(G, f, n_{k} d\right) R^{n_{k} d}$ необходимо для сходимости последовательности мер $\mu_{n_{k} d}^{f}$. Для доказательства достаточности этого условия заметим, что, пользуясь отображениями $\Phi_{k}$ (см. $\left.\S 1\right)$, можно обобшить $(7.15)$ на все сплошные цилиндры $C \subset X(G)$. Тогда в силу $(7.12),(7.14)$ для любого такого цилиндра

$$
\lim _{k \rightarrow \infty} \mu_{n_{k} d}^{f}(\bar{C})=\lim _{k \rightarrow \infty} \mu_{n_{k} d}^{f}(C)=\lim _{k \rightarrow \infty} \frac{Q_{C}\left(G, f, n_{k} d\right) R^{n_{k} d}}{Q_{X(G)}\left(G, f, n_{k} d\right) R^{n_{k} d}}=\alpha \mu^{f}(C) .
$$

Пусть $\nu$ - произвольная предельная точка последовательности мер $\mu_{n_{k} d}^{f}$ (такие точки сушествуют, так как $\bar{X}(G)$ - компактное пространство). В силу $(7.16) \nu(\bar{C})=\alpha \mu^{f}(C)$, если $C$ - сплошной цилиндр в $X(G)$. Равенство (7.13) выводится отсюда точно так же, как это было сделано (пусть и для других $\nu$ и $\alpha$ ) в конце доказательства теоремы 6.4. Теорема доказана.

7.9. СлЕДСтвИЕ. Для сходимости $\mu_{k d}^{f} \kappa \mu^{f}($ при $k \rightarrow \infty)$ необходимо и достаточно, чтобы выполнялось соотношение

$$
\lim _{k \rightarrow \infty} Q_{X(G)}(G, f, k d) R^{k d}=d .
$$

Теоремы 7.6 и 7.8 говорят о том, что меры $\mu_{k d}^{f}$ при $k \rightarrow \infty$ ведут себя во многом так же, как изученные в $\S 6$ равновесные меры, отвечающие ограничениям функции $f$ на пространства путей конечных подграфов графа $G$ : всякая предельная точка последовательности $\left\{\mu_{k d}^{f}\right\}$ имеет вид, указанный в правой части (7.13) (cp. (6.2)), а в случае невозвратной или нуль-возвратной матрицы $A_{f}$ имеет место сходимость к $\delta_{\infty}$. Однако, как мы увидим в гл. 3 , на случай положительно-возвратных матриц $A_{f}$ эта аналогия не распространяется. Сейчас мы получим одно достаточное условие сходимости $\mu_{k d}^{f}$ к $\mu^{f}$, которое будет использоваться в гл. 3.

7.10. Теорема. Пусть $f \in \mathscr{F}_{0}^{1}(G), d=d(G)$ u найдутся такие $t>0, v \in V(G)$, что

$$
q_{X(G)}^{+}\left(f-t \mathbf{1}_{C(v)}\right)<q_{X(G)}^{+}(f) .
$$

Тогда

$$
\lim _{n \rightarrow \infty} \mu_{n d}^{f}=\mu^{f}
$$

ДокАЗАТельство. Вначале покажем, что при условии (7.18) матрица $A_{f}$ устойчиво-положительна. Обозначим через $G^{v}$ главный подграф графа $G$ (см. $\left.\S 3\right)$, множеством вершин которого служит $V(G) \backslash\{v\}$. Из (7.1) непосредственно вытекает, что для любой функции $h: X(G) \rightarrow \mathbb{R}$ и любого $n \geqslant 1$

$$
Q_{X(G)}(G, h, n)=Q_{\widehat{C}(v)}(G, h, n)+Q_{X(G v)}(G, h, n),
$$


где, как и раньше, $\widehat{C}(v)=\bigcup_{k \in \mathbb{Z}} S^{k} C(v)$. Поэтому

$$
q_{X(G)}^{+}(h)=\max \left\{q_{\widehat{C}(v)}^{+}(h), q_{X\left(G^{v}\right)}^{+}(h)\right\} .
$$

Положив здесь $h=f-s \mathbf{1}_{C(v)}, 0 \leqslant s<\infty$, и заметив, что $q_{X\left(G^{v}\right)}^{+}\left(f-s \mathbf{1}_{C(v)}\right)$ не зависит от $s$, получим с учетом (7.18) неравенства

$$
\begin{aligned}
q_{X\left(G^{v}\right)}^{+}(f) & <q_{\widehat{C}(v)}^{+}(f), \\
q_{\widehat{C}(v)}^{+}\left(f-s \mathbf{1}_{C(v)}\right) & <q_{\widehat{C}(v)}^{+}(f) .
\end{aligned}
$$

При всяком $s \in[0, \infty)$ функция $-s \mathbf{1}_{C(v)}$ есть, очевидно, ограничение на $X(G)$ функции $-s \mathbf{1}_{\bar{C}(v)} \in C(\bar{X}(G))$. Поэтому в силу $(7.9)$ и леммы 7.3

$$
\begin{aligned}
q_{\widehat{C}(v)}^{+}\left(f-s \mathbf{1}_{C(v)}\right) & =q_{C(v)}^{+}\left(f-s \mathbf{1}_{C(v)}\right) \\
& =P^{\operatorname{int}}\left(X(G), S, f-s \mathbf{1}_{C(v)}\right), \quad s \in[0, \infty) .
\end{aligned}
$$

Взяв $s=t$ и $s=0$, перепишем (7.22) в виде

$$
P^{\text {int }}\left(X(G), S, f-t \mathbf{1}_{C(v)}\right)<P^{\text {int }}(X(G), S, f) .
$$

По теореме 4.4

$$
P^{\mathrm{int}}(X(G), S, h)=-\ln R\left(A_{h}\right)
$$

для любой функции $h \in \mathscr{F}_{0}^{1}(G)$. Поэтому из (7.23) (при $s=0$ ) и условия $R\left(A_{f}\right)>0$ вытекает, что $q_{X\left(G^{v}\right)}^{+}(f)<\infty$, а $(7.24)$ (с учетом $(7.23)$ и замечания 3.16$)$ позволяет заключить, что матрица $A_{f}$ устойчиво-положительна.

Вернемся теперь к неравенству (7.21). Положив

$$
\delta=q_{\widehat{C}(v)}^{+}(f)-q_{X\left(G^{v}\right)}^{+}(f),
$$

непосредственно убеждаемся, что если $\bar{g} \in C(\bar{X}(G)),\|\bar{g}\|<\delta / 2$ и $g=\left.\bar{g}\right|_{X(G)}$, то

$$
q_{X\left(G^{v}\right)}^{+}(f+g)<q_{\widehat{C}(v)}^{+}(f+g) .
$$

Это значит (см. (7.9), (7.20) и лемму 7.3), что для таких $g$

$$
q_{X(G)}^{+}(f+g)=P^{\operatorname{int}}(X(G), S, f+g) .
$$

Подставив те же $f$ и $g$ вместе с $Y=X(G)$ в (7.3) и перейдя к пределу при $n=k d \rightarrow \infty$, получим (с учетом $(7.25)$ и аналогичного равенства для $f$ )

$$
P^{\mathrm{int}}(X(G), S, f+g) \geqslant P^{\mathrm{int}}(X(G), S, f)+\int g d \nu,
$$

где $\nu$ - любая предельная точка последовательности $\left\{\mu_{n d}^{f}\right\}$. Стало быть, $\nu$ служит $\delta / 2$-субградиентом функции $P_{f}(g)=P^{\operatorname{int}}(X(G), S, f+g)$ в точке $g \equiv 0$ (см. $\left.\S 5\right)$. В таком случае согласно лемме $5.4 \nu \in \partial P_{f}(0)$. Но так как по доказанному вьше матрица $A_{f}$ устойчиво-положительна, мы знаем из теоремы 5.9 , что $\partial P_{f}(0)=\left\{\mu^{f}\right\}$. Отсюда следует доказываемое утверждение. 
7.11. СлЕДСТВИЕ. Если граф $G$ конечен и $f \in \mathscr{F}_{0}^{1}(G)$, то имеет место предельное соотношение (7.19), понимаемое в смысле слабой сходимости мер на $X(G)$.

ДокАЗАТЕЛЬСТво. Поскольку для конечного $G$ пространство $X(G)$ компактно, его можно отождествить с $\bar{X}(G)$. Очевидно, для любой функции $h: X(G) \rightarrow \mathbb{R}$

$$
Q_{X(G)}(G, h, n)=\sum_{v \in V(G)} Q_{C(v)}(G, h, n)
$$

а при $h \in \mathscr{F}_{0}^{1}(G)$ в силу леммы 7.3 и теоремы 4.4

$$
q_{X(G)}^{+}(h)=\max _{v \in V(G)} q_{C(v)}^{+}(h)=P^{\text {int }}(X(G), S, h)=-\ln R\left(A_{h}\right) .
$$

Так как конечная матрица всегда устойчиво-положительна (см. предложение 3.1.), справедливо неравенство

$$
R\left(A_{f-t \mathbf{1}_{C(v)}}\right)>R\left(A_{f}\right), \quad v \in V(G), \quad t \in(0,1) .
$$

Поэтому из (7.26) видно, что вьполняется условие (7.18), которое по теореме 7.10 влечет за собой (7.19). Следствие доказано.

Глава 3.

Динамические дзета-функции, связанные с СЦМ

Динамические дзета-функции, которьм посвящена эта глава, служат наряду с дискретными инвариантными мерами, важной характеристикой множества периодических точек любой динамической системы. Обе указанные характеристики содержат сушественную информацию об этом множестве и, тем самьм, друг о друге. Мы, в частности, используем эту связь для более точного, чем в предыдущей главе, описания асимптотики дискретных инвариантных мер. Заметим, что в случае конечных СЦМ эта задача решается без привлечения дзета-функций, причем не только для функций $f \in \mathscr{F}_{0}^{1}(G)$, к которым относится следствие 7.11 , но и для функций более общего вида (см. [43]).

\section{§. Теорема факторизации и некоторые ее следствия}

Пусть $f: X(G) \rightarrow \mathbb{R}, B \subset X(G)$ и $Q_{B}(G, f, n)<\infty$ при всех $n \geqslant 1$. Тогда можно определить степенной ряд

$$
\psi_{f, B}(z)=\sum_{n=1}^{\infty} \frac{Q_{B}(G, f, n)}{n} z^{n}=\sum_{n=1}^{\infty} \frac{z^{n}}{n} \sum_{x \in B \cap \mathrm{Fix}_{n}(G)} \exp \sum_{i=0}^{n-1} f\left(S^{i} x\right) .
$$

Если этот ряд имеет ненулевой радиус сходимости $r(G, f, B)$, то можно говорить о $\psi_{f, B}$ как о функции, аналитической в круге $|z|<r(G, f, B)$.

8.1. ОПРЕДЕЛЕНИЕ. ФУНКцИЮ

$$
\zeta_{f, B}(z)=\exp \psi_{f, B}(z)
$$


будем назьвать локальной динамической дзета-функиией ( $\zeta$-функиией), отвечающей паре $(f, B)$.

Эпитет “локальная", конечно, связан с множеством $B$, которое не обязательно совпадает со всем пространством $X(G)$. При $B=X(G)$ будем опускать как этот эпитет, так и указание на множество $B$ во всех обозначениях, относящихся к динамической дзета-функции; в частности, будем писать $\psi_{f}, \zeta_{f}$ и $r(G, f)$ вместо $\psi_{f, X(G)}, \zeta_{f, X(G)}$ и $r(G, f, X(G))$ соответственно.

Функция $\zeta_{f}$ при $f \equiv 0$ (для произвольной динамической системы) была введена Е. Артином и Б. Мазуром [37], а в общем случае - Д. Рюэлем [38].

Следуюшая важная теорема устанавливает связь между локальными $\zeta$-функциями и производяшими функциями, изучавшимися в гл. 1. Хотя по существу она не является новой (ср. [39]), нам не удалось найти в литературе ее подробного доказательства. В формулировке будут фигурировать уже встречавшееся в гл. 2 множество $\widehat{C}(v)=\bigcup_{n \in \mathbb{Z}} S^{n} C(v)$ и функция $f^{v}=\left.f\right|_{X\left(G^{v}\right)}$, где $G^{v}$ - главньй подграф графа $G$ с множеством вершин $V\left(G^{v}\right)=V(G) \backslash\{v\}$ (введенньй при доказательстве теоремы 7.10).

8.2. Теорема. Пусть $G$ - конечный или счетный ориентированный граф (не обязательно связный) $f \in \mathscr{F}_{0}^{1}(G)$ и $A_{f}$ - соответствующая матрица Больимана. Тогда для произвольной вершины $v \in V(G)$ выполняются тождества

$$
\begin{aligned}
\zeta_{f, \widehat{C}(v)}(z) & =\left[1-\varphi_{v v}^{0}\left(A_{f}, z\right)\right]^{-1}, \quad|z|<R\left(A_{f}\right), \\
\zeta_{f}(z) & =\left[1-\varphi_{v v}^{0}\left(A_{f}, z\right)\right]^{-1} \zeta_{f^{v}}(z), \quad|z|<r(G, f) .
\end{aligned}
$$

ДокАЗАТЕЛЬСТВО. При каждом $n \geqslant 1$ будем считать последовательности $x, y \in$ $\operatorname{Fix}_{n}(G)$ эквивалентньми и писать $x \sim y$, если найдется такое $k \in \mathbb{Z}$, что $S^{k} x=y$. Легко понять, что если $x=\left(x_{i}, i \in \mathbb{Z}\right) \in \widehat{C}(v) \cap \operatorname{Fix}_{n}(G)$, то $x_{i}=v$ при бесконечно многих $i<0$ и бесконечно многих $i>0$. Каждой такой последовательности $x$ поставим в соответствие числа

$$
\begin{gathered}
i^{-}(x)=\max \left\{i \leqslant 0: x_{i}=v\right\}, \quad i^{+}(x)=i^{-}(x)+n, \\
k(x)=\#\left\{i: i^{-}(x) \leqslant i<i^{+}(x), x_{i}=v\right\} .
\end{gathered}
$$

Очевидно, $k(x)=k(y)$, если $x \sim y$. Поэтому множество тех $x \in \widehat{C}(v) \cap \operatorname{Fix}_{n}(G)$, для которых $k(x)=k$, разбивается на описанные только что классы эквивалентности. Совокупность этих классов обозначим через $\mathscr{F}_{n, k}$.

Пусть $x \in \widehat{C}(v) \cap \operatorname{Fix}_{n}(G), k(x)=k$ и $i_{0}, \ldots, i_{k-1}$ определяются условиями: $i^{-}(x)=i_{0}<i_{1}<\cdots<i_{k-1}<i^{+}(x), x_{i}=v$ при $i=i_{r}, 1 \leqslant r \leqslant k-1$. Тогда по $x$ можно построить последовательность $\left(\gamma_{1}, \ldots, \gamma_{k}\right)=\tilde{\gamma}(x)$, где $\gamma_{r}=\left(x_{i_{r}}, \ldots, x_{i_{r+1}}\right)$ цикл из $\Gamma_{v v}^{0}(G), r=0, \ldots, k-1$.

Две последовательности элементов множества $\Gamma_{v v}^{0}(G)$ будем считать эквивалентными, если они получаются друг из друга циклической перестановкой. Очевидно, у эквивалентных последовательностей совпадают число и суммарная длина входящих в них циклов. Так же легко проверить, что $\tilde{\gamma}(x)$ и $\tilde{\gamma}\left(x^{\prime}\right)$ эквивалентны в том и только 
том случае, когда $x \sim x^{\prime}$. Поэтому, поставив в соответствие каждому классу эквивалентности $F \in \mathscr{F}_{n, k}$ класс эквивалентности $\alpha(F)=H(\tilde{\gamma}(x))$, содержаший последовательность $\tilde{\gamma}(x)$, где $x$ - любая точка из $F$, мы получим обратимое отображение $\alpha$ множества $\mathscr{F}_{n, k}$ на множество $\mathscr{H}_{n, k}$ классов эквивалентности последовательностей $\tilde{\gamma}=\left(\gamma_{1}, \ldots, \gamma_{k}\right) \in\left(\Gamma_{v v}^{0}(G)\right)^{k} \mathrm{c} l\left(\gamma_{1}\right)+\cdots+l\left(\gamma_{k}\right)=n$.

Сравним мошности множеств $F \in \mathscr{F}_{n, k}$ и $\alpha(F) \in \mathscr{H}_{n, k}$. Пусть $\alpha(F)=H, \tilde{\gamma} \in H$ и $m(\gamma)$ - наименьшее из таких натуральных $m$, что $\tilde{\gamma}$ можно разбить на $m$ одинаковых

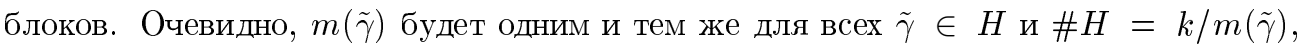
$\# F=n / m(\tilde{\gamma})$. Следовательно,

$$
\frac{\# F}{\# \alpha(F)}=\frac{n}{k}, \quad F \in \mathscr{F}_{n, k} .
$$

Теперь заметим, что если $f \in \mathscr{F}_{0}^{1}(G)$ и $A=A_{f}$, то для любых $n \geqslant 1, k=1, \ldots, n$, $F \in \mathscr{F}_{n, k}, x \in F$ и любой последовательности $\tilde{\gamma}=\left(\gamma_{1}, \ldots, \gamma_{k}\right) \in \alpha(F)$ вьполняется равенство

$$
\exp \sum_{j=0}^{n-1} f\left(S^{j} x\right)=\prod_{r=1}^{k} Q\left(A, \gamma_{r}\right)
$$

Поэтому, введя обозначения

$$
\begin{gathered}
\Gamma(n, k)=\left\{\left(\gamma_{1}, \ldots, \gamma_{k}\right) \in\left(\Gamma_{v v}^{0}(G)\right)^{k}: \sum_{r=1}^{k} l\left(\gamma_{r}\right)=n\right\}, \\
\widetilde{Q}\left(A_{f}, \tilde{\gamma}\right)=\prod_{r=1}^{k} Q\left(A, \gamma_{r}\right), \quad \tilde{\gamma} \in \Gamma(n, k),
\end{gathered}
$$

получим (см. (8.4))

$$
\begin{aligned}
Q_{\widehat{C}(v)}(G, f, n) & =\sum_{x \in \widehat{C}(v) \cap \mathrm{Fix}_{n}(G)} \exp \sum_{j=0}^{n-1} f\left(S^{j} x\right) \\
& =\sum_{k=1}^{n} \sum_{F \in \mathscr{F}_{n, k}} \sum_{x \in F} \exp \sum_{j=0}^{n-1} f\left(S^{j} x\right) \\
& =\sum_{k=1}^{n} \sum_{F \in \mathscr{F}_{n, k}} \# \sum_{\tilde{\gamma} \in \alpha(F)} \widetilde{Q}\left(A_{f}, \tilde{\gamma}\right) / \# \alpha(F) \\
& =\sum_{k=1}^{n} \frac{n}{k} \sum_{H \in \mathscr{H}_{n, k}} \sum_{\left(\gamma_{1}, \ldots, \gamma_{k}\right) \in H} \prod_{r=1}^{k} Q\left(A_{f}, \gamma_{r}\right) \\
& =n \sum_{k=1}^{n} \frac{1}{k} \sum_{\left(\gamma_{1}, \ldots, \gamma_{k}\right) \in \Gamma(n, k)} \prod_{r=1}^{k} Q\left(A_{f}, \gamma_{r}\right) \\
& =n \sum_{k=1}^{n} \frac{1}{k} \sum_{l_{1}+\cdots+l_{k}=n} \prod_{r=1}^{k} Q_{v v}^{0}\left(A_{f}, l_{r}\right) .
\end{aligned}
$$


Внутренняя сумма в последнем выражении есть, очевидно, коэффициент при $z^{n}$ в разложении функции $\left(\varphi_{v v}^{0}\left(A_{f}, z\right)\right)^{k}$ по степеням $z$.

Заметим, что

$$
\left|\varphi_{v v}^{0}\left(A_{f}, z\right)\right|<1, \quad|z|<R\left(A_{f}\right) .
$$

Если граф $G$ связен, это вытекает из п. 2 леммы 2.6, а если $G$ несвязен, те же соображения надо применить к его связной компоненте $G_{v}$, содержашей вершину $v$, и к функции $f_{v}=\left.f\right|_{X\left(G_{v}\right)}$ (может случиться, что эта связная компонента пуста, но в таком случае $\left.\varphi_{v v}^{0}\left(A_{f}, z\right) \equiv 0\right)$.

Из (8.1), (8.6) с учетом (8.7) получаем

$$
\psi_{f, \widehat{C}(v)}(z)=\sum_{k=1}^{\infty} \frac{1}{k}\left[\varphi_{v v}^{0}\left(A_{f}, z\right)\right]^{k}=-\ln \left[1-\varphi_{v v}^{0}\left(A_{f}, z\right)\right], \quad|z|<R\left(A_{f}\right) .
$$

Отсюда сразу следует (8.2) (см. определение 8.1), а для доказательства (8.3) достаточно заметить, что $r(G, f) \leqslant R\left(A_{f}\right)$ и справедливо тождество

$$
\psi_{f}(z)=\psi_{f, \widehat{C}(v)}(z)+\psi_{f v}(z), \quad|z|<r(G, f) .
$$

Теорема доказана.

8.3. СлЕДСтвИЕ [5, добавление 4]. Если граф $G$ конечен $u f \in \mathscr{F} 1(G)$, то $\zeta_{f}$ продолжается на всю комплексную плоскость как рациональная функиия. При этом $r(G, f)=R\left(A_{f}\right)$.

ДокАЗАТЕЛЬство. Применив формулу (8.3) к функции $\zeta_{f}$ и произвольной вершине $v=v_{1} \in V(G)$, можно затем заменить $v_{1}$ на любую вершину $v_{2}$ графа $G^{v_{1}}$ и снова применить (8.3), но уже к $\zeta_{f} v_{1}$ и $v_{2}$. Продолжая эту процедуру, мы в конце конщов получим тождество

$$
\zeta_{f}(z)=\prod_{i=1}^{N}\left(1-\varphi_{v_{i} v_{i}}^{0}\left(A_{f_{i}}, z\right)\right)^{-1}
$$

где $N=\# V(G), v_{1}, \ldots, v_{N}$ - занумерованные в произвольном порядке элементы множества $V(G)$ и $f_{i}$ - ограничение функции $f$ на пространство путей $X(G(i))$ графа $G(i)$, полученного из $G$ удалением всех вершин $v_{j}$ с $j<i$. Остается вспомнить, что согласно предложению 3.1 все функции $\varphi_{v_{i} v_{i}}^{0}\left(A_{f_{i}}, \cdot\right)$ рациональны (в случае, когда граф $G(i)$ несвязен, предложение 3.1 надо применить к его связной компоненте, содержашей вершину $v_{i}$; если эта компонента пуста, отвечаюшая ей $\zeta$-функция равна единице).

В $\S 10$ мы приведем другое, более явное выражение для $\zeta_{f}$ в случае конечного граф̆а $G$ и функции $f \in \mathscr{F}_{0}^{1}(G)$ (см. теорему 10.1).

8.4. ЗАмЕчАНИЕ. Легко видеть, что как формула (8.8), так и ее доказательство сохраняются и для некоторых бесконечных графов, а именно, для тех из них, где имеется конечное множество вершин $V^{\prime}$, через которое проходят все циклы. При этом роль $N$ в тождестве (8.8) должно играть число $\# V^{\prime}$. Указанным свойством обладают, в частности, все лепестковые графы (см. определение 5.14).

Теорема 8.2 позволяет вернуться к вопросу о предельном поведении последовательности $S$-инвариантных мер $\mu_{n d}^{f, \widehat{C}(v)}$, дополнив следствие 7.5 и теорему 7.8 следующим утверждением. 
8.5. СлЕДСТВИЕ. Если $f \in \mathscr{F}_{0}^{1}(G)$ и матрица $A_{f}$ положительно-возвратна, то при всех $v \in V(G)$

$$
\lim _{n \rightarrow \infty} \mu_{n d}^{f, \widehat{C}(v)}=\mu^{f}
$$

ДокАЗАТЕльство. Как уже было отмечено при доказательстве предложения 7.4, мера $\mu_{n d}^{f, \widehat{C}(v)}$ определена при всех достаточно больших $n$. Согласно $(7.2)$ для любого борелевского множества $B \subset X(G)$

$$
\begin{aligned}
\mu_{n d}^{f, \widehat{C}(v)}(B) & =\frac{Q_{B \cap \widehat{C}(v)}(G, f, n d)}{Q_{\widehat{C}(v)}(G, f, n d)} \\
& =\frac{Q_{B \cap \widehat{C}(v)}(G, f, n d) R^{n d}}{Q_{\widehat{C}(v)}(G, f, n d) R^{n d}},
\end{aligned}
$$

где $R=R\left(A_{f}\right)$. Найдем пределы числителя и знаменателя в правой части последней дроби. Начнем со знаменателя.

Из (8.1) видно, что $Q_{\widehat{C}(v)}(G, f, n d)$ есть коэффициент при $z^{n d}$ в разложении по степеням $z$ функции $z \zeta_{f, \widehat{C}(v)}^{\prime}(z) / \zeta_{f, \widehat{C}(v)}(z)$. В силу $(8.2),(2.10)$

$$
\frac{z \zeta_{f, \widehat{C}(v)}^{\prime}(z)}{\zeta_{f, \widehat{C}(v)}(z)}=z \varphi^{\prime}(z)(1+\psi(z))
$$

где $\varphi(z)=\varphi_{v v}^{0}\left(A_{f}, z\right), \psi(z)=\varphi_{v v}\left(A_{f}, z\right)$. Поэтому

$$
Q_{\widehat{C}(v)}(G, f, n d) R^{n d}=d \sum_{k=1}^{n} k Q_{v v}^{0}\left(A_{f}, k d\right) R^{k d} Q_{v v}\left(A_{f},(n-k) d\right) R^{(n-k) d}
$$

где $Q_{v v}\left(A_{f}, 0\right)$ принимается равным нулю. Поскольку матрица $A_{f}$ положительно-возвратна,

$$
\sum_{k=1}^{\infty} k d Q_{v v}^{0}\left(A_{f}, k d\right) R^{k d}=R \varphi^{\prime}(R)<\infty
$$

(см. $\S 2)$. По той же причине и в силу леммы 7.7

$$
\lim _{n \rightarrow \infty} Q_{v v}\left(A_{f}, n d\right) R^{n d}=\frac{d}{R \varphi^{\prime}(R)} .
$$

Из (8.10)-(8.12) легко выводится, что

$$
\lim _{n \rightarrow \infty} Q_{\widehat{C}(v)}(G, f, n d) R^{n d}=d .
$$

Теперь обратимся к числителю дроби в правой части (8.9). Пусть $B \subset X(G)-$ произвольный $v$-цилиндр с носителем $\{0, \ldots, l\}$ (см. определение 5.5$)$. Если $l=0$, то $B=C(v)$ и

$$
Q_{B \cap \widehat{C}(v)}(G, f, n d)=Q_{C(v)}(G, f, n d)=Q_{v v}\left(A_{f}, n d\right)
$$


Если же $l>0$, то $l=k d, k \in \mathbb{N}, B=C(\gamma)$, где $\gamma \in \Gamma_{v v}(G, l)$, и при $n>k$

$$
Q_{B}(G, f, n d)=Q\left(A_{f}, \gamma\right) Q_{C(v)}(G, f,(n-k) d)=Q\left(A_{f}, \gamma\right) Q_{v v}\left(A_{f},(n-k) d\right)
$$

В силу (8.12), (8.14), (8.15)

(8.16) $\lim _{n \rightarrow \infty} Q_{C(v) \cap \widehat{C}(v)}(G, f, n d) R^{n d}=\lim _{n \rightarrow \infty} Q_{C(v)}(G, f, n d) R^{n d}=\frac{d}{R \varphi^{\prime}(R)}$,

$$
\lim _{n \rightarrow \infty} Q_{C(\gamma) \cap \widehat{C}(v)}(G, f, n d) R^{n d}=\lim _{n \rightarrow \infty} Q_{C(\gamma)}(G, f, n d) R^{n d}=\frac{Q\left(A_{f}, \gamma\right) R^{l} d}{R \varphi^{\prime}(R)} .
$$

Правые части (8.16), (8.17) равны соответственно $d \mu^{f}(C(v))$ и $d \mu^{f}(C(\gamma))$ (см. теорему 4.6 и предложение 4.7$)$. Возврашаясь к $(8.13),(8.9)$, получаем отсюда

$$
\lim _{n \rightarrow \infty} \mu_{n d}^{f, \widehat{C}(v)}(B)=\mu^{f}(B)
$$

для любого $v$-цилиндра $B \subset X(G)$. Значит, для любой предельной точки $\nu$ последовательности мер $\mu_{n d}^{f, \widehat{C}(v)}$ и любого такого цилиндра выполняется равенство $\nu(B)=\mu^{f}(B)$. Ссылка на лемму 5.8 завершает доказательство.

8.6. СлеДСтвИЕ. Пусть $f \in \mathscr{F}_{0}^{1}(G)$ и матрица $A_{f}$ положительно-возвратна. Тогда:

1) если при некотором $v \in V(G)$ выполняется соотношение

$$
\lim _{n \rightarrow \infty} Q_{X\left(G^{v}\right)}\left(G^{v}, f^{v}\right)\left[R\left(A_{f}\right)\right]^{n}=0,
$$

$m o \lim _{n \rightarrow \infty} \mu_{n d}^{f}=\mu^{f}$

2) если $\lim _{n \rightarrow \infty} \mu_{n d}^{f}=\mu^{f}$, то (8.18) имеет место при любом $v \in V(G)$.

ДоКАЗАТЕЛЬСТВо. В силу теоремы 7.8 сходимость $\mu_{n d}^{f}$ к $\mu^{f}$ равносильна соотношению

$$
\lim _{n \rightarrow \infty} Q_{X(G)}(G, f, n d)\left[R\left(A_{f}\right)\right]^{n d}=d,
$$

которое заведомо будет вьполняться, если в его левой части заменить $Q_{X(G)}(G, f, n d)$ на $Q_{\widehat{C}(v)}(G, f, n d)$ при любом $v \in V(G)$ (см. (8.13)). Отсюда вытекают оба доказываемые утверждения, так как, очевидно, имеют место тождества

$$
\begin{aligned}
Q_{X(G)}(G, f, n d) & =Q_{\widehat{C}(v)}(G, f, n d)+Q_{X(G) \backslash \widehat{C}(v)}(G, f, n d) \\
& =Q_{\widehat{C}(v)}(G, f, n d)+Q_{X\left(G^{v}\right)}\left(G^{v}, f^{v}, n d\right), \quad v \in V(G) .
\end{aligned}
$$




\section{§. Мероморфное продолжение дзета-функций и поведение дискретных инвариантных мер}

Согласно следствию 8.3 в случае конечного графа $G$ и произвольной функции $f \in$ $\mathscr{F} 1(G)$ динамическая $\zeta$-функция $\zeta_{f}$ рациональна. При переходе к бесконечньм графам это свойство, вообше говоря, не сохраняется, и самое большее, на что можно рассчитьвать в сколько-нибудь общем случае, - это наличие у $\zeta_{f}$ мероморфного продолжения в некоторьй круг, содержаший круг сходимости ряда (8.1). Последнее свойство допускает уточнение.

9.1. ОПРЕ ДЕЛЕНИЕ. Будем говорить, что функция $\zeta_{f, B}$, где $B \subset X(G)$, в частности, функция $\zeta_{f}(z)$ допускает простейшее мероморфное продолжсение (ПМП), если она продолжается в некоторьй круг $|z|<r$, где $r>R\left(A_{f}\right)$, как мероморфная функция, у которой нет нулей, а особенности исчерпьваются простыми полюсами в точках $z_{k}=R\left(A_{f}\right) \exp (2 \pi i k / d), 0 \leqslant k \leqslant d-1$, где $d=d(G)$.

Проще всего обстоит дело с мероморфным продолжением локальных $\zeta$-функций $\zeta_{f, \widehat{C}(v)}, v \in V(G)$.

9.2. ПРЕДЛОЖЕНИЕ. Пусть $G-$ связный граф $u f \in \mathscr{F}_{0}^{1}(G)$. Тогда:

1) если матрица $A_{f}$ устойчиво-положительна, то при любом $v \in V(G)$ функция $\zeta_{f, \widehat{C}(v)}$ допускает простейшее мероморфное продолэсение;

2) если $R\left(A_{f}\right)>0$ и хотя би при одном $v \in V(G)$ функция $\zeta_{f, \widehat{C}(v)}$ допускает простейшее мероморфное продолжение, то матрица $A_{f}$ устойчиво-положительна.

ДоКАЗАТЕльСТВо. 1) В силу (8.2) и (2.10) при любом $v \in V(G)$ справедливы тождества

$$
\zeta_{f, \widehat{C}(v)}(z)=\left[1-\varphi_{v v}^{0}\left(A_{f}, z\right]^{-1}=1+\varphi_{v v}\left(A_{f}, z\right), \quad|z|<R\left(A_{f}\right)\right.
$$

первое из которых показьвает, что $\zeta_{f, \widehat{C}(v)}(z)$ продолжается в круг $|z|<R_{v v}^{0}\left(A_{f}\right)$ как мероморфная функщия, не имеюшая нулей. Если матрища $A_{f}$ устойчиво-положительна, то $R_{v v}^{0}\left(A_{f}\right)>R\left(A_{f}\right)$ (см. (2.12)) и согласно п. 1 предложения 3.5 найдется такое $r \in\left(R\left(A_{f}\right), R_{v v}^{0}\left(A_{f}\right)\right)$, что функция $\varphi_{v v}\left(A_{f}, \cdot\right)$ продолжается до мероморфной в круге $|z|<r$ функции, особенности которой исчерпьваются простьми полюсами в точках $z_{k}=R\left(A_{f}\right) \exp (2 \pi i k / d(G)), k=0, \ldots, d(G)-1$. Но в силу второго из приведенных тождеств $\zeta_{f}, \widehat{C}(v)$ имеет в круге $|z|<r$ те же особенности, что и $\varphi_{v v}\left(A_{f}, \cdot\right)$. Значит, она допускает ПМП.

2) Достаточно применить п. 2 предложения 3.5. Предложение доказано.

Изучение функции $\zeta_{f}$ начнем с простого вспомогательного утверждения.

9.3. ЛЕммА. Пусть функция $\varphi$ регулярна в круге $|z|<r, 0<r<\infty, u$

$$
z \varphi^{\prime}(z)=\sum_{n=1}^{\infty} b_{n d} z^{n d}, \quad|z|<r
$$


где $d \in \mathbb{N}$. Тогда следующие два утвержсдения равносильнь:

1) существуют такие $u \in \mathbb{R}, c \in \mathbb{R}^{+} u q \in(0,1)$, что

$$
\left|b_{n d} r^{n d}-u\right|<c q^{n d}, \quad n \geqslant 1
$$

2) существуют число $r_{1}>r$ и функиия $h$, регулярная и не имеющая нулей в круге $|z|<r_{1}$, для которых выполняется тождество

$$
e^{\varphi(z)}=\left(r^{d}-z^{d}\right)^{-u / d} h(z), \quad|z|<r,
$$

где $\left(r^{d}-z^{d}\right)^{-u / d}$ понимается как та ветвь степенной функиии, которая в нуле равна $1 / r^{u}$.

ДокАЗАТЕЛЬСтво. Пусть

$$
\varphi(z)=\sum_{n=0}^{\infty} a_{n} z^{n}, \quad|z|<r .
$$

Из (9.1) видно, что $a_{n}=b_{n} / n$ при $n=k d, k \in \mathbb{N}$ и $a_{n}=0$ при остальных $n \in \mathbb{N}$. Поэтому (9.2) можно переписать в виде

$$
\left|a_{k d}-u /\left(k d r^{k d}\right)\right|<c q^{k d} /\left(k d r^{k d}\right), \quad k \geqslant 1,
$$

a (9.4) - в виде

$$
\begin{aligned}
\varphi(z) & =a_{0}+\sum_{k=1}^{\infty}(u /(k d))(z / r)^{k d}+\sum_{k=1}^{\infty} c_{k d} z^{k d} \\
& =a_{0}-(u / d) \ln \left[1-(z / r)^{d}\right]+\sum_{k=1}^{\infty} c_{k d} z^{k d} \\
& =a_{0}-(u / d) \ln \left[1-(z / r)^{d}\right]+g(z), \quad|z|<r,
\end{aligned}
$$

где $\left|c_{k d}\right|<(c / k d)(q / r)^{k d}, k \geqslant 1$. Следовательно, функция $g$ регулярна в круге $|z|<r_{1}$ при некотором $r_{1}>r$, и мы приходим к (9.3).

Обратно, пользуясь неравенствами Коши для коэффициентов степенного ряда, нетрудно вывести (9.2) из представления (9.5), которое, в свою очередь, получается из (9.3). Лемма доказана.

Связь между существованием ПМП функции $\zeta_{f}$ и поведением последовательности дискретных инвариантных мер $\mu_{n}^{f}$ (см. $\left.\S 7\right)$ видна из следующего утверждения.

9.4. ТеоремА. Если для функции $f \in \mathscr{F}_{0}^{1}(G)$ матрица $A_{f}$ положительно-возвратна и функция $\zeta_{f}$ допускает простейшее мероморфное продолжение, то последовательность мер $\mu_{n}^{f}$ сходится к равновесной мере $\mu^{f}$.

ДоКАЗАТЕЛЬСТво. Положив $\varphi(z)=\psi_{f}(z), r=R\left(A_{f}\right), b_{n d}=Q_{X(G)}(G, f, n d)$ и воспользовавшись определениями 8.1, 9.1 и определением функции $\zeta_{f}$ (см. (8.1)), легко убедиться в справедливости равенства (9.3) с $u=d$. Согласно лемме 9.3 отсюда следует (9.2) и остается применить следствие 7.9. Теорема доказана.

Сформулируем теперь критерий существования ПМП функции $\zeta_{f}$. 
9.5. ТеОремА. Пусть $G$ - связный ориентированный граф $и \in \in \mathscr{F}_{0}^{1}(G)$. Тогда:

1) если при некоторых $v \in V(G)$ и $t>0$ выполняется неравенство

$$
r\left(G, f-t \mathbf{1}_{C(v)}\right)>r(G, f),
$$

то функиия $\zeta_{f}$ допускает простейшее мероморфное продолжение;

2) если $\zeta_{f}$ допускает простейшее мероморфное продолэсение и при некоторых $w \in V(G), s>0$ то жсе самое верно для функции $\zeta_{f-s \mathbf{1}_{C(w)}}$, то для всех $v \in V(G), t>0$ выполняется (9.6).

ДоКАЗАТЕльСТво. 1) Очевидно, при любых $v \in V(G)$ и $t>0$

$$
\varphi_{v v}^{0}\left(A_{f-t \mathbf{1}_{C(v)}}, z\right)=e^{-t} \varphi_{v v}^{0}\left(A_{f}, z\right), \quad|z|<R_{v v}^{0}\left(A_{f}\right) .
$$

Поэтому формула (8.3) для функции $f-t \mathbf{1}_{C(v)}$ принимает вид

$$
\zeta_{f-t 1_{C(v)}}(z)=\left[1-e^{-t} \varphi_{v v}^{0}\left(A_{f}, z\right)\right]^{-1} \zeta_{f v}(z)
$$

Оба сомножителя в ее правой части разлагаются в степенные ряды, коэффициенты которых неотрицательны, а радиусы сходимости соответственно равны $R\left(A_{f-t \mathbf{1}_{C(v)}}\right)$ и $r\left(G^{v}, f^{v}\right)$ (где $G^{v}$ - граф, впервые введенньй в доказательстве теоремы 7.10). Отсюда следует, что

$$
r\left(G, f-t \mathbf{1}_{C(v)}\right)=\min \left\{R\left(A_{f-t \mathbf{1}_{C(v)}}\right), r\left(G^{v}, f^{v}\right)\right\} \text { при } t \geqslant 0 .
$$

Пусть теперь для некоторых $v=v_{0} \in V(G)$ и $t=t_{0}>0$ вьполняется неравенство (9.6). Тогда, используя (9.9) при этих $v$ и $t$, а затем при $v=v_{0}, t=0$, получаем

$$
r(G, f)=R\left(A_{f}\right)<r\left(G^{v}, f^{v}\right) .
$$

Кроме того, можно утверждать, что матрица $A_{f}$ устойчиво-положительна, так как в противном случае при всех $v$ и $t$ вьполнялись бы равенства $R\left(A_{f-t \mathbf{1}_{C(v)}}\right)=R\left(A_{f}\right)$ (см. (9.7) и п. 2 леммы 2.6) и $r\left(G, f-t \mathbf{1}_{C(v)}\right)=r(G, f)$ (см. (9.9)), что по условию невозможно. Следовательно, $R_{v v}^{0}\left(A_{f}\right)>R\left(A_{f}\right)$ и к функции $\varphi(\cdot)=\varphi_{v v}^{0}\left(A_{f}, \cdot\right)$ можно применить лемму 3.4 , в силу которой функщия $\left[1-\varphi_{v v}^{0}\left(A_{f}, \cdot\right)\right]^{-1}$ допускает ПМП в некоторьй круг $|z|<r$, где $R\left(A_{f}\right)<r<R_{v v}^{0}\left(A_{f}\right)$, причем функция $\zeta_{f v}(\cdot)$ регулярна и не обрашается в нуль (очевидно, всегда можно считать, что $r \leqslant r\left(G^{v}, f^{v}\right)$ ). Отсюда следует доказьваемое утверждение.

2 ) Вначале покажем, что матрица $A_{f}$ устойчиво-положительна. Из условия вытекает, что $r(G, f)=R\left(A_{f}\right)$, так как в противном случае $r(G, f)<R\left(A_{f}\right)($ см. $(9.9))$ и функция $\zeta_{f}$ имела бы особенности в круге $|z|<R\left(A_{f}\right)$, что невозможно при наличии ПМП. Точно так же доказьвается, что $r\left(G, f-s \mathbf{1}_{C(w)}\right)=R\left(A_{f-s \mathbf{1}_{C(w)}}\right)$. Кроме

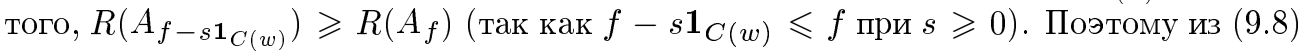
и (8.3) получаем

$$
\frac{\zeta_{f}(z)}{\zeta_{f-s \mathbf{1}_{C(w)}}(z)}=\frac{1-e^{-s} \varphi_{w w}^{0}\left(A_{f}, z\right)}{1-\varphi_{w w}^{0}\left(A_{f}, z\right)}, \quad|z|<R\left(A_{f}\right) .
$$


Обозначим левую часть (9.11) через $g(z)$. Наличие ПМП у $\zeta_{f}$ и $\zeta_{f-s} \mathbf{1}_{C(w)}$ приводит к тому, что функция $g$ аналитична в круге $|z|<R\left(A_{f}\right)$ и допускает мероморфное продолжение в круг $|z|<r$ при некотором $r>R\left(A_{f}\right)$. Так как функция $\varphi_{w w}^{0}\left(A_{f}, \cdot\right)$, а вслед за ней и $\varphi_{w w}\left(A_{f}, \cdot\right)$ рационально выражаются через $g$, обе эти функции допускают мероморфное продолжение в круг $|z|<r($ см. (9.11) и (2.10)). Но в силу п. 2 предложения 3.5 это влечет за собой устойчивую положительность матришы $A_{f}$.

Вследствие только что доказанного при всех $v \in V(G)$ выполняется неравенство $R\left(A_{f}\right)<R_{v v}^{0}\left(A_{f}\right)$. Применив к функции $\varphi_{w w}^{0}\left(A_{f}, \cdot\right)$ лемму 3.4 и предположение о наличии ПМП у функции $\zeta_{f}$, можно утверждать, что при некотором $r_{v}>R\left(A_{f}\right)$ функция $\zeta_{f} v$ при некотором $r_{v}>R\left(A_{f}\right)$ допускает не имеюшее нулей аналитическое продолжение в круг $|z|<r_{v}$. В сопоставлении с установленным выше равенством $r(G, f)=R\left(A_{f}\right)$ это означает, что $r\left(G^{v}, f^{v}\right)>r(G, f)$. Еше раз воспользовавшись устойчивой положительностью $A_{f}$ и равенством $(9.8)$, отсюда уже легко вьвести, что $r\left(G, f-t \mathbf{1}_{C(v)}\right)>r(G, f)$ при всех $t \geqslant 0$. Теорема доказана.

9.6. ЗАмЕчАниЕ. Легко видеть, что если для данных $f=f_{1}$ и $t=t_{1}$ выполняется неравенство (9.6), то при достаточно малом $\varepsilon>0$ оно будет выполняться для того же $v$ и всех $t>0, f \in \mathscr{F}_{0}^{1}(G)$, удовлетворяюших условию $\left|t-t_{1}\right|<\varepsilon,\left\|f-f_{1}\right\|<\varepsilon$, где $\|\cdot\|$ - норма в $C(X(G))$. В таком случае согласно п. 1 теоремы 9.5 функция $\zeta_{f}$ при всех упомянутых $f$ будет обладать ПМП.

В связи с теоремой 9.5 естественно возникают два связанных друг с другом вопроса: 1) можно ли обратить утверждение из п. 1 этой теоремы? 2) можно ли доказать, что устойчивая положительность матрищы $A_{f}$ (вытекающая, как мы убедились, из условий обоих пунктов теоремы) в действительности эквивалентна сушествованию ПМП у функции $\zeta_{f}$ ? Сейчас мы увидим, что ответы на оба эти вопроса - отрицательные, а заодно выясним, каким реально может быть предел последовательности дискретных инвариантных мер $\mu_{n}^{f}$ в случае, когда матрица $A_{f}$ устойчиво-положительна (см. теорему 7.8).

Начнем с одной общей конструкции. Пусть при $n=1,2, \ldots$ задан конечньй граф $G_{n}$ с множеством вершин $V_{n}$, причем все $V_{n}$ имеют обшую точку $v$ и $V_{i} \cap V_{j}=\{v\}$ при любых $i \neq j$. Объединение всех $G_{n}$ будем называть обобщеннылм лепестковым графом с центральной вершиной $v$ и обобшенньми лепестками $G_{1}, G_{2}, \ldots$ Для этого графа введем обозначение $\left(\left\{G_{n}\right\}, v\right)$. В частном случае, когда все $G_{n}$ - простые циклы, мы получим обычньй лепестковьй граф (см. определение 5.14).

9.7. ПримеР. Рассмотрим обобщенньй лепестковьй граф $\left(\left\{G_{n}\right\}, v\right)$, в котором каждый обобшенный лепесток $G_{n}$ - это обычный лепестковый граф̆ с центральной вершиной $v_{n} \neq v$, состояший из двух лепестков; предположим, что их длины равны соответственно $l_{n}$ и $2 l_{n}$, причем $v$ принадлежит второму из этих лепестков и служит его серединой, т.е. имеет номер $l_{n}$ при естественной нумерации вершин вдоль лепестка. Очевидно, в этом случае граф $\left(\left\{G_{n}\right\}, v\right)$ однозначно определяется последовательностью $\left\{l_{n}\right\}$, а поскольку нумерация обобшенных лепестков $G_{n}$ никакой роли не играет, для задания $\left(\left\{G_{n}\right\}, v\right)$ достаточно указать числа

$$
h(k)=\#\left\{n: l_{n}=k\right\}, \quad k=1,2, \ldots
$$

Зафиксировав произвольное $\beta>0$ и произвольные целые $m, r>1$, положим

$$
h(k)= \begin{cases}0, & 1 \leqslant k \leqslant r-1, \\ {\left[\beta m^{k} / k\right],} & k \geqslant r\end{cases}
$$


(здесь $[\cdot]$ - целая часть числа) и полученньй таким образом граф обозначим через $G_{\beta, m, r}$. В качестве $f$ возьмем функцию $f_{0}$, тождественно равную нулю на $X\left(G_{\beta, m, r}\right)$. Соответствуюшая ей матрица Больцмана есть, очевидно, матрица смежности графа $G_{\beta, m, r}$. Обозначим ее через $A_{\beta, m, r}$. Легко проверить, что

$$
\begin{gathered}
\varphi_{v v}^{0}\left(A_{\beta, m, r}, z\right)=\sum_{k=1}^{\infty} h(k) \frac{z^{2 k}}{1-z^{k}}=\sum_{k=r}^{\infty}\left[\frac{\beta m^{k}}{k}\right] \frac{z^{2 k}}{1-z^{k}}, \\
R_{v v}^{0}\left(A_{\beta, m, r}\right)=m^{-1 / 2}
\end{gathered}
$$

Пользуясь тем, что в точке $z=1 / m$ ряд (9.13) сходится, подберем (при фиксированных $\beta, m)$ значение параметра $r$ так, чтобы разность $\Delta=1-\varphi_{v v}^{0}\left(A_{\beta, m, r}, 1 / m\right)$ была положительна. Разложив $\Delta$ по степеням $1 / m$ :

$$
\Delta=\sum_{n=1}^{\infty} p_{n} m^{-n}, \quad p_{n} \in \mathbb{Z}^{+}, \quad 0 \leqslant p_{n}<m
$$

построим лепестковьй граф̆ с центральной вершиной $v$ (той же, что и у $G_{\beta, m, r}$ ), в котором число лепестков длины 2 равно $m p_{1}+p_{2}$ (напомним, что по определению в лепестковом графе нет лепестков длины 1 ), число лепестков длины $n>2$ равно $p_{n}$ и нет обших с $G_{\beta, m, r}$ вершин, отличных от $v$. Объединение построенного граф̆а с $G_{\beta, m, r}$ обозначим через $G$. Очевидно, $G$ будет по-прежнему обобшенным лепестковым графом с центральной вершиной $v$. Пусть $A$ - его матрища смежности (матрица Больцмана функции $\left.f_{0} \equiv 0\right)$ и $\zeta_{A}=\zeta_{f_{0}}$ (здесь мы несколько отступаем от принятых ранее обозначений; поскольку и в дальнейшем, до конща этого параграфа, будут встречаться лишь функции $f$, тождественно равные нулю, мы будем всюду заменять $f$ и пару $(f, G)$ соответствуюшей матрицей смежности).

Из построения (см. также (9.12)) непосредственно вытекает, что

$$
\begin{aligned}
& \varphi_{v v}^{0}(A, z)=\sum_{k=r}^{\infty}\left[\frac{\beta m^{k}}{k}\right] \frac{z^{2 k}}{1-z^{k}}+\sum_{k=1}^{\infty} p_{k} z^{k} \\
& R_{v v}^{0}(A)=\frac{1}{\sqrt{m}}, \quad R(A)=\frac{1}{m}, \quad d(G)=1,
\end{aligned}
$$

откуда, в частности, видно, что матрища $A$ устойчиво-положительна.

Вычислим $\zeta_{A}(z)$. Пусть, аналогично предыдущему, $A^{v}$-матрища смежности граффа $G^{v}$, полученного из $G$ в результате удаления вершины $v$ и всех связанных с ней ребер. Используя обозначения $[c]$ и $\{c\}$ соответственно для целой и дробной частей числа 
$c \in \mathbb{R}$, получаем

$$
\begin{aligned}
\psi\left(A^{v}, z\right)= & \sum_{k=r}^{\infty}\left[\frac{\beta m^{k}}{k}\right] \sum_{n=1}^{\infty} \frac{z^{n k}}{n} \\
= & \sum_{k=r}^{\infty} \frac{\beta m^{k}}{k} \sum_{n=1}^{\infty} \frac{z^{n k}}{n}-\sum_{k=r}^{\infty}\left\{\frac{\beta m^{k}}{k}\right\} \sum_{n=1}^{\infty} \frac{z^{n k}}{n} \\
= & \beta \sum_{k=r}^{\infty} \frac{m^{k}}{k} z^{k}+\beta \sum_{k=r}^{\infty} \frac{m^{k}}{k} \sum_{n=2}^{\infty} \frac{z^{n k}}{n}-\sum_{k=r}^{\infty}\left\{\frac{\beta m^{k}}{k}\right\} \sum_{n=1}^{\infty} \frac{z^{n k}}{n} \\
= & -\beta \ln (1-m z)-\beta \sum_{k=1}^{r-1} \frac{m^{k}}{k} z^{k} \\
& +\beta \sum_{k=r}^{\infty} \frac{m^{k}}{k} z^{2 k} \sum_{n=0}^{\infty} \frac{z^{n k}}{n+2}-\sum_{k=r}^{\infty}\left\{\frac{\beta m^{k}}{k}\right\} \sum_{n=1}^{\infty} \frac{z^{n k}}{n}
\end{aligned}
$$

Нетрудно проверить, что последние три из четырех слагаемых в правой части (9.17) представляют собой аналитические функщии в круге $|z|<m^{-1 / 2}$. Поэтому (см. (9.16), (8.3) и лемму 3.4)

$$
\zeta_{A}(z)=(1-m z)^{-1-\beta} u(z)
$$

где функция $u$ регулярна и не обрашается в нуль в круге $|z|<m^{-1 / 2}$. Таким образом, в этом примере функция $\zeta_{A}$, несмотря на устойчивую положительность матрицы $A$, может иметь в точке $R(A)=1 / m$ как полюс произвольного порядка $>1$ (при натуральном $\beta$ ), так и изолированную особенность многозначного характера. Очевидно, такая функция не допускает ПМП.

Из (9.18) с помощью леммы 9.3 (см. также доказательство теоремы 9.4) получаем

$$
\left|Q(A, n) m^{-n}-1-\beta\right|<c q^{n}, \quad n \geqslant 1,
$$

где $c>0, q \in(0,1)$. Согласно теореме 7.8 это означает, что последовательность мер $\mu_{n}^{A}$ сходится к мере $\alpha \mu^{A}+(1-\alpha) \delta_{\infty}$, где $\alpha=(1+\beta)^{-1}$ можно сделать произвольным числом из $(0,1)$, выбрав подходящее $\beta$.

Несложное видоизменение описанной только что конструкции, а именно, замена в $(9.12)\left[\beta m^{k} / k\right]$ на $m^{k}$, приводит к устойчиво-положительной матрице $A_{1}$, для которой $d\left(A_{1}\right)=1, R\left(A_{1}\right)=1 / m$ и

$$
\psi_{A_{1}^{v}}(z)=(1-m z)^{-1}+\eta(z), \quad|z|<m^{-1 / 2}
$$

где функция $\eta$ регулярна в круге $|z|<m^{-1 / 2}$. С помошю леммы 3.4 и теоремы 8.2 можно убедиться, что функция $\zeta_{A_{1}}$ имеет в точке $1 / m$ сушественную особенность и что

$$
z \psi_{A_{1}}^{\prime}(z)=m z(1-m z)^{-2}+m z(1-m z)^{-1}+\eta_{1}(z)
$$

где функция $\eta_{1}$ регулярна в круге $|z|<r$ при некотором $r>1 / m$. Это позволяет, рассуждая аналогично предыдушему, прийти к равенству

$$
\lim _{n \rightarrow \infty} Q\left(A_{1}, n\right) m^{-n}=\infty,
$$


из которого согласно теореме 7.8 следует, что $\lim _{n \rightarrow \infty} \mu_{n}^{A_{1}}=\delta_{\infty}$. Таким образом, в классе устойчиво-положительных матриц $A_{f}$ последовательность мер $\mu_{n}^{f}$ может сходиться к любой из мер вида $\alpha \mu^{f}+(1-\alpha) \delta_{\infty}$, где $\alpha \in[0,1]$.

Теперь приведем пример, показьвающий, что функция $\zeta_{f}$ может иметь простейшее мероморфноепродолжение и в том случае, когда матрица $A_{f}$ не является положительно-возвратной и тем более - устойчиво-положительной.

9.8. ПримеР. Пусть $A_{2}$ - матрица смежности ориентированного графа $G_{2}$ с множеством вершин $V_{2}=\mathbb{Z}^{+}$и множеством ребер

$$
E_{2}=\{(0,1),(i, i-1),(i, i+1), i=1,2, \ldots\}
$$

(очевидно, $G_{2}$ описьвает простейшее случайное блуждание на $\mathbb{Z}^{+}$с отражением в нуле). Пользуясь тождеством (2.10), легко получить соотношение

$$
\varphi_{00}^{0}\left(A_{2}, z\right)=z^{2}\left(1+\varphi_{00}\left(A_{2}, z\right)\right)=\frac{z^{2}}{1-\varphi_{00}^{0}\left(A_{2}, z\right)},
$$

вследствие которого,

$$
\varphi_{00}^{0}\left(A_{2}, z\right)=\frac{1}{2}\left(1-\left(1-4 z^{2}\right)^{1 / 2}\right)
$$

(выбор знака перед корнем определяется условием $\varphi_{00}^{0}\left(A_{2}, 0\right)=0$ ).

Рассмотрим лепестковый граф̆ $G_{3}$ с центральной вершиной $w$, в котором число лепестков длины $n$ равно $2 Q_{00}^{0}\left(A_{2}, n\right), n=1,2, \ldots$ (напомним, что $Q_{00}^{0}\left(A_{2}, n\right)$ - коэффициент при $z^{n}$ в разложении $\varphi_{00}^{0}\left(A_{2}, z\right)$ по степеням $\left.z\right)$. Пусть $A_{3}$ - матрица смежности этого графа. Из построения видно, что $d\left(G_{3}\right)=2$ и

$$
\varphi_{w w}^{0}\left(A_{3}, z\right)=1-\left(1-4 z^{2}\right)^{1 / 2} .
$$

Следовательно,

$$
R\left(A_{3}\right)=1 / 2, \quad \varphi_{w w}^{0}\left(A_{3}, R\left(A_{3}\right)\right)=1,\left.\quad \frac{d}{d z} \varphi_{w w}^{0}\left(A_{3}, z\right)\right|_{z=R\left(A_{3}\right)}=\infty
$$

и в силу $(9.20),(8.3)$

$$
\zeta_{A_{3}}(z)=\left(1-\varphi_{w w}^{0}\left(A_{3}, z\right)\right)^{-1}=\left(1-4 z^{2}\right)^{-1 / 2} .
$$

Пусть $A_{4}$ - матрища смежности тензорного произведения $G_{4}=G \otimes G_{3}$ (напомним, что тензорное произведение $G^{\prime} \otimes G^{\prime \prime}$ графов $G^{\prime}$ и $G^{\prime \prime}$ определяется как граф̆ с множеством вершин $V\left(G^{\prime}\right) \times V\left(G^{\prime \prime}\right)$, ребрами которого служат пары $\left(\left(v_{1}^{\prime}, v_{1}^{\prime \prime}\right),\left(v_{2}^{\prime}, v_{2}^{\prime \prime}\right)\right)$, где $\left(v_{1}^{\prime}, v_{2}^{\prime}\right) \in E\left(G^{\prime}\right),\left(v_{1}^{\prime \prime}, v_{2}^{\prime \prime}\right) \in E\left(G^{\prime \prime}\right)$, и что матрица смежности тензорного произведения графов равна тензорному произведению матрищ смежности сомножителей, см. [51]). Легко проверить, что граф̆ $G_{4}$ связен, $d\left(G_{4}\right)=2$ и

$$
Q_{(v, w)(v, w)}\left(A_{4}, n\right)=Q_{v v}(A, n) Q_{w w}\left(A_{3}, n\right), \quad n \geqslant 1 .
$$

Поскольку матрища $A$ устойчиво-положительна и $d(G)=1, R(A)=1 / m$ (см. пример 9.7), из теоремы 2.7 и леммы 7.7 вытекает, что последовательность 
$\left\{Q_{v v}(A, n) m^{-n}, n=1,2, \ldots\right\}$ имеет положительньй предел. Тогда (см. (9.23), а затем $(9.15)$ и $(9.21))$

$$
\begin{aligned}
\frac{1}{R\left(A_{4}\right)} & =\varlimsup_{n \rightarrow \infty}\left(Q_{(v, w)(v, w)}\left(A_{4}, n\right)\right)^{1 / n} \\
& =\lim _{n \rightarrow \infty}\left(Q_{v v}(A, n)\right)^{1 / n} \varlimsup_{n \rightarrow \infty}\left(Q_{w w}\left(A_{3}, n\right)\right)^{1 / n} \\
& =\frac{1}{R(A) R\left(A_{3}\right)}=2 m,
\end{aligned}
$$

т.е. $R\left(A_{4}\right)=1 /(2 m)$. Еще раз используя лемму 7.7 (вместе с (9.21)), получаем

$$
\lim _{n \rightarrow \infty} Q_{(v, w)(v, w)}\left(A_{4}, n\right)(2 m)^{-n}=0,
$$

откуда следует, что матрица $A_{4}$ не является положительно-возвратной.

Исследуем функцию $\zeta_{A_{4}}$. Для этого прежде всего заметим, что в силу (9.22)

$$
z \frac{\zeta_{A_{3}}^{\prime}(z)}{\zeta_{A_{3}}(z)}=z \psi_{A_{3}}^{\prime}(z)=\frac{4 z^{2}}{1-4 z^{2}}=\sum_{n=1}^{\infty} 4^{n} z^{2 n}
$$

т.е. (см. (8.1))

$$
Q\left(A_{3}, 2 n\right)=4^{n}, \quad Q\left(A_{3}, 2 n+1\right)=0, \quad n=1,2, \ldots
$$

Очевидно, для коэффициентов $Q\left(A_{4}, 2 n\right)$ справедливо тождество, аналогичное (9.23), а именно:

$$
Q\left(A_{4}, 2 n\right)=Q(A, 2 n) Q\left(A_{3}, 2 n\right), \quad n=1,2, \ldots .
$$

Вместе с (9.19) и (9.25) оно дает:

$$
\left|Q\left(A_{4}, 2 n\right)(2 m)^{-2 n}-(1+\beta)\right|<c q^{n}, \quad n=1,2, \ldots,
$$

где $c>0, q \in(0,1)$. Положим теперь $\beta=1$. Так как по доказанному $d\left(G_{4}\right)=2$, $R\left(A_{4}\right)=1 /(2 m)$, можно применить лемму 9.3 , в силу которой функция $\zeta_{A_{4}}$ допускает ПМП за пределы круга $|z|<1 /(2 m)$ (это продолжение имеет простые полюса в точках $1 /(2 m)$ и $-1 /(2 m))$.

\section{§10. Дзета-функция и спектральные свойства неотрицательных матриц}

Основные результаты этого параграффа были опубликованы без доказательства в [49].

В $\S 8$ мы упомянули явную формулу для ращиональной функции, которая служит продолжением на всю комплексную плоскость функции $\zeta_{f}$ в случае, когда граф $G$ конечен и $f \in \mathscr{F}_{0}^{1}(G)$. Она имеет следуюший вид.

10.1. Теорема [40]. В указанных условиях

$$
\zeta_{f}(z)=\left[\operatorname{det}\left(I-z A_{f}\right)\right]^{-1}, \quad|z|<r(G, f),
$$


где I - единичная матрица того же порядка, что и $A_{f}$.

Сравнивая формулы (10.1) и (8.3), нетрудно заметить, что если конечный граф̆ $G$ связен, то множество собственных чисел матрищы $A_{f}$, лежаших на окружности $|z|=$ $\lambda\left(A_{f}\right)=1 / R\left(A_{f}\right)$ (см. замечание 2.3 ), совпадает с множеством нулей каждой из функщий $z \mapsto 1-\varphi_{v v}^{0}\left(A_{f}, 1 / z\right), v \in V(G)$, лежаших на той же окружности. Структура первого из этих множеств хорошо известна (см., например, [18]): оно состоит из точек $z_{k}=R\left(A_{f}\right) \exp (2 \pi i k / d(G)), k=0, \ldots, d(G)-1$, и все эти собственные числа простые. Лемма 3.4 показьвает, что точно так же вьглядит множество нулей функции $z \mapsto 1-\varphi_{v v}^{0}\left(A_{f}, z\right)$ в случае любой (не обязательно конечной) неразложимой неотрицательной матрищы $A_{f}$.

Следуюшее утверждение вытекает из теорем 8.2 и 10.1.

10.2. СлЕДСТВИЕ. Если $G$ - конечный связный граф и $f \in \mathscr{F}_{0}^{1}(X(G))$, то для любой вершины $v \in V(G)$ множество собственных чисел матрицы $A_{f}$, лежащих в области $|z|>1 / R_{v v}^{0}\left(A_{f}\right)$, переводится в множество нулей функиии $1-\varphi_{v v}^{0}\left(A_{f}, \cdot\right)$ преобразованием $z \rightarrow 1 / z$, причем кратности соответствующих друг другу собственных чисел и нулей совпадают.

ДоКАЗАТЕЛЬСТВо. Из (10.1) видно, что множество ненулевых собственных чисел матрицы $A_{f}$ совпадает с множеством полюсов функции $z \mapsto \zeta_{f}(1 / z)$ и кратность каждого собственного числа равна порядку отвечающего ему полюса. Поэтому с учетом теоремы 8.2 и отсутствия нулей у функции $\zeta_{f}$ в круге $|z|<r\left(G^{v}, f^{v}\right)$ достаточно доказать, что

$$
r\left(G^{v}, f^{v}\right) \geqslant R_{v v}^{0}\left(A_{f}\right)
$$

Так как граф $G^{v}$ (как и $G$ ) конечен, из сравнения формул $(2.1),(2.3)$ и (8.1) получаем равенство $r\left(G^{v}, f^{v}\right)=\min _{A^{\prime}} R\left(A^{\prime}\right)$, где минимум берется по всем неразложимьм подматрицам матрицы $A^{v}$. Если таких подматриц нет, то $\lambda\left(A_{f}\right)=0$ и $(10.2)$, очевидно, вьполняется. Предположив, что неразложимые подматрицы $A^{\prime} \subset A_{f}$ сушествуют, зафиксируем любую из них. Пусть $\gamma^{-}-$кратчайший $G$-путь, ведуший из $v$ в множество вершин $V\left(G^{\prime}\right)$ графа $G^{\prime}$, отвечаюшего подматрице $A^{\prime}$, и $v^{\prime} \in V\left(G^{\prime}\right)$ - конец этого пути. Пусть, далее, $\gamma^{+}-$кратчайший $G$-путь, ведущий из $v^{\prime}$ в $v$. Поставив в соответствие каждому пути $\gamma^{\prime} \in \Gamma_{v^{\prime} v^{\prime}}\left(G^{\prime}\right)$ путь $\pi\left(\gamma^{\prime}\right)=\gamma^{-} \gamma^{\prime} \gamma^{+}$, мы получим, как легко понять, обратимое отображение $\pi: \Gamma_{v^{\prime} v^{\prime}}\left(G^{\prime}\right) \rightarrow \Gamma_{v v}^{0}(G)$, удовлетворяющее условиям

$$
l\left(\pi\left(\gamma^{\prime}\right)\right)=l\left(\gamma^{\prime}\right)+l\left(\gamma^{-}\right)+l\left(\gamma^{+}\right), \quad Q\left(A_{f}, l\left(\pi\left(\gamma^{\prime}\right)\right)\right)=Q\left(A_{f}, \gamma^{-}\right) Q\left(A^{\prime}, \gamma^{\prime}\right) Q\left(A_{f}, \gamma^{+}\right)
$$

Отсюда следует, что

$$
Q_{v^{\prime} v^{\prime}}\left(A^{\prime}, n\right) \leqslant \frac{Q_{v v}^{0}\left(A, n+l\left(\gamma^{-}\right)+l\left(\gamma^{+}\right)\right)}{Q\left(A_{f}, \gamma^{-}\right) Q\left(A_{f}, \gamma^{+}\right)}
$$

а потому

$$
R_{v v}^{0}\left(A_{f}\right) \leqslant R\left(A^{\prime}\right)
$$

и, значит, $r\left(G^{v}, f^{v}\right) \geqslant R_{v v}^{0}\left(A_{f}\right)$, т.е. (10.2) вьполняется и в этом случае. Следствие доказано. 
Напомним, что в $\S 3$ устойчиво-положительные матрищы были охарактеризованы в терминах максимальных собственных чисел их подматрищ. При этом для любой бесконечной неотрицательной матрицы $A$ мы определили величину $\lambda(A)$ как точную верхнюю грань максимальных собственных чисел $\lambda\left(A^{\prime}\right)$ конечных подматриц $A^{\prime} \subset A$. Следуюшая теорема показывает, что в этом случае в спектре матрищы $A^{\prime}$ между собственными числами, лежашими на окружности $|z|=\lambda\left(A^{\prime}\right)$ (заметим, что множество таких собственных чисел не обязательно сводится к одному $\lambda\left(A^{\prime}\right)$ ), и остальньм спектром имеется зазор, величина которого отделена от нуля равномерно по тем $A^{\prime} \subset A$, для которых $\lambda\left(A^{\prime}\right)$ близко к $\lambda(A)$.

10.3. ТЕОРемА (о щели в спектре). Для всякой неразложимой устойчиво-положительной бесконечной матрицы $A$ найдется такое $\delta>0$, что все собственные числа $\lambda$ любой конечной подматрицы $A^{\prime} \subset A$, удовлетворяющие условию $|\lambda|<\left|\lambda\left(A^{\prime}\right)\right|$, лежсат в круге $|z|<\lambda(A)-\delta$.

ДокАЗАТЕЛЬСТво. Прежде всего заметим, что утверждение теоремы достаточно доказать для неразложимых подматриц матрицы $A$, так как спектр всякой матрицы есть объединение спектров ее неразложимых компонент.

Зафиксируем произвольную вершину $v$ графа $G=G_{A}$. По определению $R_{v v}^{0}(A)$ функция $\varphi_{v v}^{0}(A, \cdot)$ аналитична в круге $|z|<R_{v v}^{0}(A)$, причем устойчивая положительность матрицы $A$ влечет за собой неравенство $R_{v v}^{0}(A)>R(A)$. По той же причине $\varphi_{v v}^{0}(A, R(A))=1$ (см. п. 2 леммы 2.6), а так как коэффициенты $Q_{v v}^{0}(A, n)$ неотрицательны, функщия $1-\varphi_{v v}^{0}(A, \cdot)$ не обрашается в нуль в круге $|z|<R(A)$. Поэтому найдется такое $r \in\left(R(A), R_{v v}^{0}(A)\right)$, что множество нулей этой функции в круге $|z| \leqslant r$ исчерпывается нулями, лежашими на окружности $|z|=R(A)$. Отсюда, в частности, следует, что число

$$
\varepsilon=\min _{|z|=r}\left|1-\varphi_{v v}^{0}(A, z)\right|
$$

положительно.

Воспользуемся теперь представлением

$$
\varphi_{v v}^{0}(A, z)=\sum_{\gamma \in \Gamma_{v v}^{0}(G)} Q(A, \gamma) z^{l(\gamma)}, \quad|z|<R_{v v}^{0}(A)
$$

(см. (2.7)), чтобы найти конечный набор путей $\Gamma=\left\{\gamma_{1}, \ldots, \gamma_{k}\right\} \subset \Gamma_{v v}^{0}(G)$, для которого

$$
\sum_{\gamma \in \Gamma_{v v}^{0}(G) \backslash \Gamma} Q(A, \gamma) r^{l(\gamma)}<\varepsilon
$$

Пусть $\mathscr{A}$-множество всех неразложимых конечных подматриц $A^{\prime} \subset A$ и $\mathscr{A}^{\prime}$ состоит из тех $A^{\prime} \in \mathscr{A}$, для которых все $\gamma \in \Gamma$ являются $G^{\prime}$-путями, где $G^{\prime}=G_{A^{\prime}}$. Если $A^{\prime} \in \mathscr{A}^{\prime}$, то $\Gamma_{v v}^{0}\left(G^{\prime}\right) \backslash \Gamma \subset \Gamma_{v v}^{0}(G) \backslash \Gamma$ и вследствие $(10.5)$

$$
\max _{|z|=r}\left|\left(1-\varphi_{v v}^{0}\left(A^{\prime}, z\right)\right)-\left(1-\varphi_{v v}^{0}(A, z)\right)\right|<\varepsilon .
$$


Поскольку функция $1-\varphi_{v v}^{0}\left(A^{\prime}, \cdot\right)$ аналитична в круге $|z|<R_{v v}^{0}\left(A^{\prime}\right)$ и $R_{v v}^{0}\left(A^{\prime}\right) \geqslant$ $R_{v v}^{0}\left(A^{\prime}\right)$, по теореме Руше из $(10.3),(10.6)$ следует, что функции $1-\varphi_{v v}^{0}(A, \cdot)$ и $1-\varphi_{v v}^{0}\left(A^{\prime}, \cdot\right)$ имеют в круге $|z|<r$ одинаковое число нулей. В силу леммы 3.4 и выбора $r$ множество нулей первой из них исчерпывается $d(G)$ нулями первого порядка, лежашими на окружности $|z|=R(A)$. По той же лемме вторая функция имеет $d\left(G^{\prime}\right)$ простых нулей, лежаших на окружности $|z|=R\left(A^{\prime}\right)$, и не имеет нулей в круге $|z|<R\left(A^{\prime}\right)$. Так как, очевидно, $d\left(G^{\prime}\right) \geqslant d(G)$, отсюда следует, что $R\left(A^{\prime}\right) \leqslant r$, $d\left(G^{\prime}\right)=d(G)$ и все нули функции $1-\varphi_{v v}^{0}\left(A^{\prime}, \cdot\right)$ в круге $|z| \leqslant r$ лежат на окружности $R\left(A^{\prime}\right)$. Согласно следствию 10.2 это значит, что если $\lambda$ - собственное число матрищы $A^{\prime}$, удовлетворяющее условию $1 / r \leqslant|\lambda|<\lambda(A)$, то $|\lambda|=\lambda\left(A^{\prime}\right)$. Так как $\lambda\left(A^{\prime}\right)<\lambda(A)$, отсюда следует, что если $|\lambda|<\lambda\left(A^{\prime}\right)$, то $|\lambda|<1 / r=\lambda(A)-\delta_{0}$, где $\delta_{0}>0$.

Рассмотрим теперь множество $\mathscr{A}_{j}, j=1, \ldots, k$, состоящее из всех $A^{\prime} \in \mathscr{A}$, для которых $v \in V\left(G^{\prime}\right)$, но путь $\gamma_{j} \in \Gamma$ не является $G^{\prime}$-путем. При любом $z>0$

$$
\varphi_{v v}^{0}\left(A^{\prime}, z\right) \leqslant \varphi_{v v}^{0}(A, z)-Q(A, \gamma) z^{l(\gamma)}, \quad A^{\prime} \in \mathscr{A}_{j},
$$

и в то же время

$$
\varphi_{v v}^{0}(A, R(A))=\varphi_{v v}^{0}\left(A^{\prime}, R\left(A^{\prime}\right)\right)=1, \quad A^{\prime} \in \mathscr{A}_{j} .
$$

Поэтому сушествует такое $\delta_{j}>0$, что при всех $A^{\prime} \in \mathscr{A}_{j}$ вьполняется неравенство $R\left(A^{\prime}\right)>R(A)+\delta_{j}$. Следовательно, при всех $A^{\prime} \in \mathscr{A}_{j}$ функция $1-\varphi_{v v}^{0}\left(A^{\prime}, \cdot\right)$ не имеет нулей в круге $|z| \leqslant R(A)+\delta_{j}$ и согласно следствию 10.2 все собственные числа матришы $A^{\prime}$ лежат в круге

$$
|z|<\lambda(A)-(\lambda(A))^{2} \delta_{j} /\left(1+\lambda(A) \delta_{j}\right) .
$$

Пусть, наконец, множество $\mathscr{A}_{k+1}$ состоит из из тех $A^{\prime} \in \mathscr{A}$, для которых $v \notin V\left(G^{\prime}\right)$. Если $A^{\prime} \in \mathscr{A}_{k+1}$, то $A^{\prime} \subset A^{v}$ (напомним, что $A^{v}$ получается из $A$ удалением строки и столбца с индексом $v)$. В силу теоремы 3.15 и замечания $3.16 \lambda\left(A^{v}\right)<\lambda(A)-\delta_{k+1}$, где $\delta_{k+1}>0$. Следовательно, все собственные числа матриц $A^{\prime} \in \mathscr{A}_{k+1}$ лежат в круге $|z|<\lambda(A)-\delta_{k+1}$.

Поскольку $\mathscr{A}=\bigcup_{j=0}^{k+1} \mathscr{A}_{j}$, доказанное можно подытожить следующем образом: все собственные числа $\lambda$ любой матрицы $A^{\prime} \in \mathscr{A}$, за исключением, быть может, тех, для которых $|\lambda|=\lambda\left(A^{\prime}\right)$, лежат в круге $|z|<\lambda(A)-\delta$, где

$$
\delta=\min \left\{\delta_{0}, \delta_{k+1},(\lambda(A))^{2} \delta_{j} /\left(1+\lambda(A) \delta_{j}\right), 1 \leqslant j \leqslant k\right\} .
$$

Теорема доказана.

В некоторых случаях теорему о щели в спектре можно доказать и для бесконечной матришы, рассматривая ее как линейный оператор в соответствуюшем пространстве последовательностей. Мы ограничимся случаем, когда $A$ - бесконечная симметрическая матрища. Легко видеть, что если $\lambda(A)<\infty$, то $A$ порождает ограниченный линейньй оператор в пространстве $l^{2}(\mathbb{N})$, состоящем из функций $\xi: \mathbb{N} \rightarrow \mathbb{R}$, удовлетворяюших условию $\sum_{n \in \mathbb{N}} \xi^{2}(n)<\infty$; для этого оператора также будем употреблять обозначение $A$. 
10.4. ТеоремА. Если бесконечная симметрическая матрица $A$ с $\lambda(A)<\infty$ устойчиво-положительна, то в интервале $(\lambda(A)-\delta, \lambda(A))$, где $\delta$ - константа из теоремь 10.3, нет точек спектра оператора $A$.

ДокАЗАТЕльство. Пусть $\left\{G^{(n)}\right\}$ - исчерпывающая последовательность конечных главных подграфов графа $G=G_{A}$ (см. определение 3.9$)$. Заменив в матрице $A$ все элементы $a_{i j}$, для которых $(i, j) \notin E\left(G^{(n)}\right)$, нулями, мы получим последовательность бесконечных матриц $A^{(n)}$. Отвечаюшие им операторы (которые мы отождествляем с $\left.A^{(n)}\right)$, как легко понять, сходятся в сильной операторной топологии к $A$. Тогда всякий интервал, имеюший непустое пересечение со спектром $A$, должен обладать аналогичным свойством и по отношению к спектру $A^{(n)}$ при достаточно большом $n$ (см., например, $[49$, гл. $10, \S 7])$. Но спектр оператора $A^{(n)}$ вне точки $z=0$ есть не что иное, как множество ненулевых собственных чисел конечной матрицы, полученной из $A^{(n)}$ удалением всех нулевых строк и столбцов. Поэтому ссылкана теорему 10.3 завершает доказательство.

\section{Глава 4.}

\section{Некоторые специальные классы СЦМ}

Предыдушие главы содержали ряд теорем, условия которых довольно трудно проверить в случае СЦМ, отвечающей произвольному графу $G$. Цель данной главы указать классы графов, для которых эта задача сушественно облегчается.

\section{$\S$ 11. СЦМ с регулярным множеством периодических орбит}

11.1. ОПРЕДЕЛЕНИЕ. Будем говорить, что множество $\operatorname{Per}(G)$ периодических точек СЦМ $(X(G), S)$ регулярно относительно семейства $\mathscr{F}$, состояшего из функций $f: X(G) \rightarrow \mathbb{R}$, если $r(G, f)=R(G, f, v)$ для всех $f \in \mathscr{F}$ и $v \in V(G)$.

Если для $G, \mathscr{F}$ выполняются условия, сфформулированные в определении 11.1 , и $f \in \mathscr{F}$, то вместо $R(G, f, v), v \in V(G)$, можно писать $R(G, f)$, что мы в дальнейшем и будем делать, не оговаривая этого каждый раз специально.

Как и в предыдущих главах, будем употреблять обозначение $\mathscr{F}_{0}^{m}(G)$ для множества функций $f: X(G) \rightarrow \mathbb{R}$, постоянных на цилиндрах с носителем $[0, m], m \in \mathbb{Z}^{+}$ (см. определение 1.10). Все функции из $\mathscr{F}_{0}^{m}(G)$, очевидно, непрерьвны. Множество непрерьвных функций $f$, постоянных на "цилиндрах с носителем $[0, \infty)$ ", т.е. таких, что $f(x)=f\left(x^{\prime}\right)$, если $x, x^{\prime} \in X(G)$ и $x_{i}=x_{i}^{\prime}$ при $i \geqslant 0$, обозначим через $\mathscr{F}_{0}^{\infty}(G)$. Потребовав дополнительно, чтобы все рассматриваемые функшии были ограничены сверху, получим соответственно множества $\mathscr{B}_{0}^{m}(G)$ и $\mathscr{B}_{0}^{\infty}(G)$.

Пусть, далее, $\operatorname{Reg}_{0}^{m}$ (соответственно $\left.B \operatorname{Reg}_{0}^{m}\right), m \in \mathbb{Z}^{+} \cup\{\infty\},-$ совокупность графов $G$, для которых множество $\operatorname{Per}(G)$ регулярно относительно $\mathscr{F}_{0}^{m}(G)$ (соответственно относительно $\left.\mathscr{B} \mathscr{F}_{0}^{m}(G)\right)$.

Введение понятия регулярности оправдьвается следуюшим утверждением.

11.2. Теорема. Eсли (а) $G \in \operatorname{Reg}_{0}^{1}$ (соответственно $\left.G \in B \operatorname{Reg}_{0}^{1}\right)$ u $f \in \mathscr{F}_{0}^{1}(G)$ (соответственно $f \in \mathscr{B}_{0}^{1}(G)$ ), то функиия $1 / \zeta_{f}$ аналитически продолжается в круг $|z|<R^{*}\left(A_{f}\right)$, где $R^{*}\left(A_{f}\right)=\sup _{v \in V(G)} R_{v v}^{0}\left(A_{f}\right)$ (cм. (3.2)); если, кроме того, (б) матрица $A_{f}$ устойчиво-положительна, то әта функиия имеет в точках

$$
z_{k}=R\left(A_{f}\right) \exp (2 \pi i k / d(G)), \quad k=0, \ldots, d(G)-1,
$$


нули первого порядка.

ДокАЗАтельство. Пусть $f \in \mathscr{F}_{0}^{1}(G)$ (соответственно $f \in \mathscr{B}_{0}^{1}(G)$ ). Тогда это верно и для $f-t \mathbf{1}_{C(v)}$ при любых $v \in V(G)$ и $t \in \mathbb{R}$. Значит, по условию регулярности мы имеем

$$
r\left(G, f-t \mathbf{1}_{C(v)}\right)=R\left(G, f-t \mathbf{1}_{C(v)}\right), \quad v \in V(G), \quad t \in \mathbb{R} .
$$

Очевидно также, что

$$
\lim _{t \rightarrow \infty} r\left(G, f-t \mathbf{1}_{C(v)}\right) \leqslant r\left(G^{v}, f\right)
$$

и, следовательно,

$$
r\left(G^{v}, f\right) \geqslant \lim _{t \rightarrow \infty} R\left(G, f-t \mathbf{1}_{C(v)}\right)
$$

В силу предложения 3.2 найдется такое $v=v^{*} \in V(G)$, что $R^{*}\left(A_{f}\right)=R_{v^{*}}^{0} v^{*}\left(A_{f}\right)$. $\mathrm{C}$ помошью соотношения (9.7) и леммы 2.6 легко понять, что

$$
\lim _{t \rightarrow \infty} R\left(G, f-t \mathbf{1}_{C\left(v^{*}\right)}\right)=R_{v^{*} v^{*}}^{0}\left(A_{f}\right)=R^{*}\left(A_{f}\right) .
$$

Используя (11.1), получаем отсюда неравенство

$$
r\left(G^{v^{*}}, f\right) \geqslant R^{*}\left(A_{f}\right)
$$

из которого видно, что функция $1 / \zeta_{f^{*}}$ аналитична в круге $|z|<R^{*}\left(A_{f}\right)$. По определению в этом круге аналитична и функция $1-\varphi_{v^{*} v^{*}}^{0}\left(A_{f}, \cdot\right)$. Вследствие (8.3) отсюда вытекает первое утверждение теоремы. Что же касается второго, то оно моментально получается из леммы 3.4. Теорема доказана.

11.3. ЗАмЕчАниЕ. С помошю отображения $m$-редукции утверждение доказанной теоремы легко обобшить на функции из $\mathscr{F}_{0}^{m}(G)\left(\right.$ соответственно из $\left.\mathscr{B} \mathscr{F}_{0}^{m}(G)\right)$ при любом $m \in \mathbb{N}$.

11.4. СлЕДСТВИЕ. Если выполнены условия (а) и (б) теоремы 11.2, то функиия $\zeta_{f}$ допускает ПМП и $\mu_{n}^{f} \rightarrow \mu^{f}$ (см. теорему 9.4).

Теперь попытаемся сформулировать условия регулярности, которые были бы более наглядными, чем ее определение. Для всякого $G$-пути $\gamma$ обозначим через $V(\gamma)$ множество вершин, составляюших этот путь. Пусть $\Gamma_{n}(G), n \in \mathbb{Z},-$ совокупность $G$-циклов длины $n$. Для всякого $n \in \mathbb{N}$, при котором $\Gamma_{n}(G) \neq \varnothing$, введем множество $\mathscr{D}_{n}(G)$, состояшее из всех таких подмножеств $D \subset V(G)$, что $D \cap V(\gamma) \neq \varnothing$ для любого $\gamma \in \Gamma_{n}(G)$. Положим

$$
\begin{aligned}
b_{n}(G) & =\min \left\{\# D: D \in \mathscr{D}_{n}(G)\right\}, \\
b(G) & =\varlimsup_{n \rightarrow \infty} n^{-1} \ln b_{n}(G) .
\end{aligned}
$$

Очевидно, $b(G)$ принимает значения в $\mathbb{R}^{+} \cup\{\infty\}$. 
11.5. ОПРеДЕЛЕНИЕ. Назовем $b(G)$ показателем роста области прохождения циклов граффа $G$. Будем говорить, что эта область растет медленно, если $b(G)=0$.

Следуюшее предложение обобшает теорему 1 из [51].

11.6. ПРЕДЛОЖЕНИЕ. Если область прохождения ииклов связного графа $G$ растет медленно, то $G \in \operatorname{Reg}_{0}^{1}$.

ДокАЗАТЕЛЬСТВо. При каждом $n$, для которого $b_{n}(G) \neq \varnothing$, зафииксируем какое-нибудь множество $D_{n} \in \mathscr{D}_{n}(G)$, удовлетворяюшее условию $\# D_{n}=b_{n}(G)$. Если $f \in \mathscr{F}_{0}^{1}(G)$, то, очевидно,

$$
Q_{X(G)}(G, f, n) \leqslant \sum_{v \in D_{n}} Q_{\widehat{C}(v)}(G, f, n) \leqslant \sum_{v \in D_{n}} n Q_{C(v)}(G, f, n) .
$$

При всех $v$ и $n$ справедливо также неравенство

$$
Q_{C(v)}(G, f, n) \leqslant\left[R\left(A_{f}\right)\right]^{-n} \text {. }
$$

В самом деле, из определения легко вьводится, что

$$
Q_{C(v)}(G, f, k n) \geqslant\left[Q_{C(v)}(G, f, n)\right]^{k}, \quad k \in \mathbb{N},
$$

и если бы нашлись $v$ и $n$, для которых $Q_{C(v)}(G, f, n)>\left[R\left(A_{f}\right)\right]^{-n}$, это привело бы к противоречию:

$$
\begin{aligned}
{\left[R\left(A_{f}\right)\right]^{-1}=} & \varlimsup_{m \rightarrow \infty}\left[Q_{C(v)}(G, f, m)\right]^{1 / m} \\
& \geqslant \varlimsup_{k \rightarrow \infty}\left[Q_{C(v)}(G, f, k n)\right]^{1 / k n} \geqslant \varlimsup_{n \rightarrow \infty}\left[Q_{C(v)}(G, f, n)\right]^{1 / n}>\left[R\left(A_{f}\right)\right]^{-1} .
\end{aligned}
$$

Подставив (11.3) в (11.2), после очевидных преобразований и взятия верхнего предела получим

$$
-\ln r(G, f) \leqslant b(G)-\ln R\left(A_{f}\right),
$$

а так как по условию $b(G)=0$ (см. определение 11.5$)$, это означает, что $r(G, f) \geqslant$ $R\left(A_{f}\right)$. С другой стороны, всегда справедливо обратное (нестрогое) неравенство. В результате мы убеждаемся, что $r(G, f)=R\left(A_{f}\right)$. Предложение доказано.

Наложив дополнительное условие на геометрию графа $G$, можно получить гораздо более сильное свойство регулярности.

Пусть, как и раньше, $V(G)$ и $E(G)$ - соответственно множество вершин и множество ребер ориентированного графа $G$. Положим

$$
e^{+}(G)=\sup _{v \in V(G)} \#\left\{v^{\prime} \in V(G):\left(v, v^{\prime}\right) \in E(G)\right\} .
$$

Если $e^{+}(G)<\infty$, граф $G$ назьвается равномерно локально-конечным вперед.

11.7. ПРЕДЛОЖЕНИЕ. Если связный граф $G$ равномерно локально-конечен вперед и $b(G)=0$, mо $G \in \operatorname{Reg}^{\infty}$. 
ДокАЗАТЕЛЬСТво. Если функции $f: X(G) \rightarrow \mathbb{R}$ и $g: X(G) \rightarrow \mathbb{R}$ удовлетворяют условию $\sup _{x \in X(G)}|f(x)-g(x)| \leqslant \varepsilon$, то $|\ln r(G, f)-\ln r(G, g)| \leqslant \varepsilon$ и $\mid \ln R(G, f, v)-$ $\ln R(G, g, v) \mid \leqslant \varepsilon$ при всех $v \in V(G)$. Поэтому достаточно доказать, что $G \in \operatorname{Reg}_{0}^{m}$ при всех $m \in \mathbb{N}$. Но для $m=1$ это уже доказано (см. предложение 11.6), а в случае $m>1$ можно применить отображение $\Phi_{m}$ (см. $\left.\S \S 1,2\right)$. Легко понять, что

$$
b_{n}\left(\Phi_{k} G\right) \leqslant e^{+}(G) b_{n}\left(\Phi_{k-1} G\right), \quad k \geqslant 2, \quad n \geqslant 1 .
$$

Следовательно,

$$
b_{n}\left(\Phi_{m} G\right) \leqslant\left[e^{+}(G)\right]^{m-1} b_{n}(G),
$$

откуда с учетом условий $e^{+}(G)<\infty$ и $b(G)=0$ получаем $b\left(\Phi_{m} G\right)=0$. Теперь воспользуемся предложением 11.6, согласно которому $\Phi_{m} G \in \operatorname{Reg}_{0}^{1}$. Чтобы вывести отсюда интересуюшее нас включение $G \in \operatorname{Reg}_{0}^{m}$, достаточно заметить, что

$$
\Phi_{m} \mathscr{F}_{0}^{m}(G)=\mathscr{F}_{0}^{1}\left(\Phi_{m} G\right)
$$

и что для всякой функции $f \in \mathscr{F}_{0}^{m}(G)$ выполняются соотношения

$$
R\left(\Phi_{m} G, \Phi_{m} f\right)=R(G, f, v), \quad v \in V(G), \quad r\left(\Phi_{m} G, \Phi_{m} f\right)=r(G, f)
$$

по поводу первого из них см. следствие 2.14 , а второе вытекает из определения. Предложение доказано.

\section{§12. СЦМ с конечной областью прохождения циклов}

В этом параграфе мы будем пользоваться некоторыми понятиями и обозначениями, введенными в $\S 11$. При одном сушественном, но зато вполне наглядном ограничении на геометрию графа $G$ мы усилим ряд утверждений, доказанных вьше. При этом $G$ будет всегда предполагаться связным.

12.1. ОПРеДЕЛЕНИЕ. Будем говорить, что граф $G$ имеет конечную область прохождения ииклов, если найдется конечное множество $V \subset V(G)$, которое содержит хотя бы одну вершину из каждого $G$-цикла. Всякое такое $V$ будем называть областью прохождения ииклов графа $G$.

Примером граф̆а с конечной областью прохождения циклов может служить любой лепестковый граф (см. определение 5.14).

Предложение 11.6 показьвает, что все графы с конечной областью прохождения циклов регулярны относительно $\mathscr{F}_{0}^{1}(G)$. Легко понять, что если $G$ не является локально-конечньм вперед, то $\Phi_{m} G$ при некотором $m>1$ уже не будет иметь конечной области прохождения циклов. Тем не менее, справедливо следующее утверждение.

12.2. Теорема. Всякий граф с конечной областью прохождения циклов регулярен относительно $\mathscr{B} \mathscr{F}_{0}^{m}(G)$ при всех $m \geqslant 1$. 
ДокАЗАТЕЛЬСТвО. Пусть $V=\left\{v_{1}, \ldots, v_{k}\right\}$ - любая область прохождения циклов графа $G$. Из ее определения следует, что при всяком $n \geqslant 1$

$$
\operatorname{Fix}_{n}(G)=\bigcup_{i=1}^{k}\left(\widehat{C}\left(v_{i}\right) \cap \operatorname{Fix}_{n}(G)\right),
$$

где, как и раньше, $\widehat{C}\left(v_{i}\right)=\bigcup_{n \in \mathbb{Z}} S^{n} C\left(v_{i}\right)$. Поэтому для всякой функции $f: X(G) \rightarrow \mathbb{R}$

$$
Q_{X(G)}(G, f, n) \leqslant \sum_{i=1}^{k} Q_{\widehat{C}\left(v_{i}\right)}(G, f, n) \leqslant \sum_{i=1}^{k} n Q_{C\left(v_{i}\right)}(G, f, n) .
$$

Если $f \in \mathscr{B}_{0}^{m}(G), m>1$, то согласно предложению 2.13

$$
Q_{C\left(v_{i}\right)}(G, f, n) \leqslant B_{i} Q_{C\left(\gamma_{i}\right)}\left(G, f, n+b_{i}\right), \quad i=1, \ldots, k,
$$

где $\gamma_{i}-$ произвольный $G$-путь длины $m$, выходящий из $v_{i}$, а $B_{i}$ и $b_{i}$ могут зависеть от этого пути, но не от $n$. Поэтому

$$
r(G, f) \geqslant \min _{1 \leqslant i \leqslant k} R\left(\Phi_{m} G, \Phi_{m} f, \tilde{v}_{i}\right),
$$

где $\tilde{v}_{i}$-вершина графа $\Phi_{m} G$, отождествляемая с путем $\gamma_{i}$. Но в силу предложения 2.13

$$
R\left(\Phi_{m} G, \Phi_{m} f, \tilde{v}_{i}\right)=R(G, f, v)
$$

при всех $i=1, \ldots, k$ и $v \in V(G)$. Следовательно,

$$
r(G, f) \geqslant R(G, f, v), \quad v \in V(G) .
$$

Так как обратное неравенство очевидно, отсюда вытекает доказываемое утверждение.

12.3. ЗАмЕчАнИЕ. Согласно предложению 11.6 эта теорема останется верной, если в ее формулировке заменить $\mathscr{B} \mathscr{F}_{0}^{m}(G), m>1$, на $\mathscr{F}_{0}^{m}(G)$ при $m=1$. Можно показать, однако, что при $m=2$ это уже не так.

Теорема 12.2 и следствие 11.4 приводят к заключению, что если $G$-граф̆ с конечной областью прохождения циклов, $f \in \mathscr{B} \mathscr{F}_{0}^{m}(G)$ и матрица $A_{\Phi_{m} f}$ устойчиво-положительна, то $\mu_{n}^{f} \rightarrow \mu^{f}$. Сейчас мы обобшим это утверждение, заменив устойчивую положительность положительной возвратностью, которая по теореме 4.6 равносильна сушествованию меры $\mu^{f}$. Для этого нам потребуется следуюшая простая лемма.

12.4. Лемма. Если $f \in \mathscr{F}_{0}^{1}(G)$ и матрица $A_{f}$ положительно-возвратна, то

$$
\sum_{n} n Q_{C(v)}\left(G, f-t \mathbf{1}_{C(v)}, n\right)\left[R\left(A_{f}\right)\right]^{n}<\infty .
$$


ДокАЗАТЕЛЬСтво. Левая часть (12.1) лишш множителем отличается от ряда, определяющего производную $\varphi_{v v}^{\prime}\left(A_{f-t 1_{C(v)}}, z\right)$ в точке $z=R\left(A_{f}\right)$. Если матрица $A_{f}$ устойчиво-положительна, то $R\left(A_{f-t \mathbf{1}_{C(v)}}\right)>R\left(A_{f}\right)$, т.е. $R\left(A_{f}\right)$ лежит в круге сходимости степенного ряда для $\varphi_{v v}\left(A_{f-t \mathbf{1}_{C(v)}}, z\right)$. Поэтому выполняется (12.1). Если же $A_{f}$ неустойчиво-положительна, то

$$
\varphi_{v v}^{0}\left(A_{f}, R\left(A_{f}\right)\right)=1,\left.\quad \frac{d}{d z} \varphi_{v v}^{0}\left(A_{f}, z\right)\right|_{z=R\left(A_{f}\right)}<\infty
$$

(см. $\S 2)$, а так как

$$
\varphi_{v v}^{0}\left(A_{f-t \mathbf{1}_{C(v)}}, z\right)=e^{-t} \varphi_{v v}^{0}\left(A_{f}, z\right),
$$

где $e^{-t}<1$, из $(2.10)$ следует, что $\varphi_{v v}^{\prime}\left(A_{f-t \mathbf{1}_{C(v)}}, R\left(A_{f}\right)\right)<\infty$, и мы снова приходим к (12.1). Предложение доказано.

12.5. ТЕОРЕМА. Пусть $G$ - граф с конечной областью прохождения ииклов и $f \in \mathscr{F}_{0}^{1}(G) \cup \mathscr{B}_{0}^{m}(G), m \geqslant 1$. Тогда:

1) $R(G, f, v)$ не зависит от $v \in V(G)$;

2) если $0<R(G, f, v)<\infty$ и существует равновесная мера $\mu^{f}$, то $\mu_{n}^{f} \rightarrow \mu^{f}$ при $n \rightarrow \infty$.

ДоКАЗАТЕЛЬСТВО. В силу предложения 11.6 и теоремы 12.2 для всякой функции $f \in \mathscr{F}_{0}^{1}(G) \cup \mathscr{B} \mathscr{F}_{0}^{m}(G)$ выполняется равенство $R(G, f, v)=r(G, f), v \in V(G)$, и можно положить $R(G, f)=r(G, f)$.

Пусть сначала $f \in \mathscr{F}_{0}^{1}(G)$. Тогда, как мы знаем, существование меры $\mu^{f}$ равносильно положительной возвратности матрищы $A_{f}$ (см. теорему 4.6), а сходимость $\mu_{n}^{f}$ к $\mu^{f}$ равносильна соотношению (7.17) (см. следствие 7.9). Из (8.1) и определения 8.1 видно, что $Q_{X(G)}(G, f, n d)$, где $d$ - период графа $G$, есть не что иное, как коэффициент при $z^{n d}$ в разложении функции $z \mapsto z\left(\ln \zeta_{f}(z)\right)^{\prime}$ по степеням $z$. В силу $(8.3) Q_{X(G)}(G, f, n d)$ можно представить в виде суммы аналогичных коэффициентов для функций $z \mapsto z\left(\ln \left[1-\varphi_{v v}^{0}\left(A_{f}, z\right)\right]^{-1}\right)^{\prime}$ и $z \mapsto z\left(\ln \zeta_{f v}(z)\right)^{\prime}$, где $v$ - произвольная вершина графа $G$. Эти коэффициенты соответственно равны $Q_{\widehat{C}(v)}(G, f, n d)$ и $Q_{X(G) \backslash \widehat{C}(v)}(G, f, n d)$. Поскольку

$$
\left(\ln \left[1-\varphi_{v v}^{0}\left(A_{f}, z\right)\right]^{-1}\right)^{\prime}=\left[\varphi_{v v}^{0}\left(A_{f}, z\right)\right]^{\prime}\left[1-\varphi_{v v}^{0}\left(A_{f}, z\right)\right]^{-1},
$$

из леммы 7.7 и представления коэффициентов левой части этого тождества в виде свертки коэффициентов двух сомножителей, стояших в правой части, легко вьвести, что

$$
\lim _{n \rightarrow \infty} Q_{\widehat{C}(v)}(G, f, n d) R^{n d}=d,
$$

где $R=R(G, f)$ (нужно воспользоваться тем, что вследствие положительной возвратности матрищы $A_{f}$ производная $\left.\frac{d}{d z} \varphi_{v v}^{0}\left(A_{f}, z\right)\right|_{z=R}$ конечна $)$. Поэтому достаточ-
но доказать, что

$$
\lim _{n \rightarrow \infty} Q_{X(G) \backslash \widehat{C}(v)}(G, f, n) R^{n}=0 .
$$


Из определения ясно, что при $n \geqslant 1, t>0$

$$
Q_{X(G) \backslash \widehat{C}(v)}(G, f, n) \leqslant Q_{X(G)}\left(G, f-t \mathbf{1}_{C(v)}, n\right) .
$$

Пусть $\left\{v_{1}, \ldots, v_{k}\right\}$ - произвольная область прохождения циклов граффа $G$. Тогда

$$
Q_{X(G)}\left(G, f-t \mathbf{1}_{C(v)}, n\right) \leqslant \sum_{i=1}^{k} n Q_{C\left(v_{i}\right)}\left(G, f-t \mathbf{1}_{C(v)}, n\right),
$$

и с помошью леммы 12.4 мы получаем (12.2).

Пусть теперь $f \in \mathscr{B}_{0}^{m}(G)$ при $m>1$. Тогда $f_{m} \in \mathscr{F}_{0}^{1}\left(G_{m}\right)$, где $G_{m}=\Phi_{m} G, f_{m}=$ $\Phi_{m} f$, и так как существует мера $\mu^{f}$, матрица $A_{f_{m}}$ положительно-возвратна. Взяв в $(12.3)\left(G_{m}, f_{m}\right)$ вместо $(G, f)$, получим

$$
Q_{X\left(G_{m}\right) \backslash \widehat{C}(\tilde{v})}\left(G_{m}, f_{m}, n\right) \leqslant Q_{X\left(G_{m}\right)}\left(G_{m}, f_{m}-t \mathbf{1}_{C(\tilde{v})}, n\right),
$$

где $\tilde{v}$ - произвольная вершина графа $G_{m}$. Однако мы не можем написать для $G_{m}$ и $f_{m}$ аналог оценки $(12.4)$, поскольку $G_{m}$ уже не имеет, вообще говоря, конечной области прохождения циклов. Поэтому поступим следующим образом. Пусть, как и раньше, $\left\{v_{1}, \ldots, v_{k}\right\}$ - какая-либо область прохождения циклов графа $G$. Для каждого $i \in[1, k]$ зафиксируем произвольньй путь $\gamma_{i}$ длины $m-1$, выходящий из $v_{i}$, и обозначим вершину графа $G_{m}$, отвечающую этому пути, через $\tilde{v}_{i}$. В качестве $\tilde{v}$ возьмем, например, $\tilde{v}_{1}$. По определению

$$
\begin{aligned}
Q_{X\left(G_{m}\right)}\left(G_{m}, f_{m}-t \mathbf{1}_{C\left(\tilde{v}_{1}\right)}, n\right) & =Q_{X(G)}\left(G, f-t \mathbf{1}_{C\left(\gamma_{1}\right)}, n\right) \\
& \leqslant \sum_{i=1}^{k} n Q_{C\left(v_{i}\right)}\left(G, f-t \mathbf{1}_{C\left(\gamma_{1}\right)}, n\right)
\end{aligned}
$$

где $C\left(\gamma_{1}\right)=\left\{x \in X(G):\left(x_{0}, \ldots, x_{m-1}\right)=\gamma_{1}\right\}$. Предложение 2.13 позволяет утверждать, что при достаточно большом $n$

$$
\begin{aligned}
Q_{C\left(v_{i}\right)}\left(G, f-t \mathbf{1}_{C\left(\gamma_{1}\right)}, n\right) & \leqslant B_{i} Q_{C\left(\gamma_{i}\right)}\left(G, f-t \mathbf{1}_{C\left(\gamma_{1}\right)}, n+b_{i}\right) \\
& =B_{i} Q_{C\left(\tilde{v}_{i}\right)}\left(G_{m}, f_{m}-t \mathbf{1}_{C\left(\tilde{v}_{1}\right)}, n+b_{i}\right)
\end{aligned}
$$

где $B_{i}$ и $b_{i}$ не зависят от $n$. Неравенство (12.5) после подстановки в него (12.6), куда, в свою очередь, подставлено (12.7), принимает вид

$$
Q_{X\left(G_{m}\right) \backslash \widehat{C}\left(\tilde{v}_{1}\right)}\left(G_{m}, f_{m}, n\right) \leqslant n \sum_{i=1}^{k} B_{i} Q_{C\left(\tilde{v}_{i}\right)}\left(G_{m}, f_{m}-t \mathbf{1}_{C\left(\tilde{v}_{1}\right)}, n+b_{i}\right) .
$$

В силу леммы 12.4 отсюда вытекает, что

$$
\lim _{n \rightarrow \infty} Q_{X\left(G_{m}\right) \backslash \widehat{C}\left(\tilde{v}_{1}\right)}\left(G_{m}, f_{m}, n\right) R^{n}=0
$$

Тем самьм теорема доказана. 


\section{СПИСОК ЛИТЕРАТУРЫ}

[1] Синай Я. Г. Гиббсовские меры в эргодической теории // УМН 1972. Т. 27. № 4. С. 21-64.

[2] Bowen R. Equilibrium States and the Ergodic Theory of Anosov Diffeomorphisms. V. 470. Berlin: Springer-Verlag, 1975 (Lect. Notes in Math.).

[3] Ruelle D. A variational formulation of equilibrium statistical mechanics and the Gibbs phase rule // Comm. Math. Phys. 1967. V. 5. P. 324-329.

[4] Ruelle D. Statistical mechanics of a one-dimensional lattice gas // Comm. Math. Phys. 1968. V. 9. P. 267-278.

[5] Ruelle D. Thermodynamic formalism. Reading, MA: Addison-Wesley, 1978.

[6] Walters P. A variational principle for the pressure of continuous transformations // Amer. J. Math. 1976. V. 97. P. 937-971.

[7] Walters P. Ergodic Theory. Introductory Lectures. V. 458. Berlin: Springer-Verlag, 1975 (Lect. Notes Math.).

[8] Lanford O. E. Entropy and equilibrium states in classical statistical mechanics. Statistical mechanics and mathematical problems // Lect. Notes in Phys. 1973. V. 20. P. 1-113.

[9] Parry W. Intrinsic Markov chains // Trans. Amer. Math. Soc. 1964. V. 112. P. 55-65.

[10] Гуревич Б. М. Топологическая энтропия счетной цепи Маркова // Докл. АН СССР. 1969. Т. 187. № 4. С. $715-718$.

[11] Гуревич Б. М. Энтропия сдвига и марковские меры в пространстве путей счетного графа // Докл. АН СССР. 1970. Т. 192. № 5. С. 963-965.

[12] Песин Я. Б., Пицкель Б.С. Топологическое давление и вариационный принцип для некомпактных множеств // Функцион. анализ и его прилож. 1984. Т. 18. № 4. С. 50-63.

[13] Bowen R. Entropy for group endomorphisms and homogeneous spaces // Trans. Amer. Math. Soc. 1971. V. 153. P. 401-414.

[14] Bowen R. Topological entropy for noncompact sets // Trans. Amer. Math. Soc. 1973. V. 184. P. $125-136$.

[15] Заргарян А. С. Вариационньй принцип для топологического давления в случае цепи Маркова со счетным числом состояний // Матем. заметки. 1986. Т. 40. №6. С. 749-761.

[16] Гуревич Б. М. Вариационньй принцип для одномерных решетчатых гиббсовских случайных полей со счетным числом состояний // Докл. АН СССР. 1978. Т. 241. С. 749-752.

[17] Salama I. A. Topological entropy and recurrence of countable chains // Pacific J. Math. 1988. V. 134. № 2. P. 325-341.

[18] Гантмахер Ф. Р. Теория матриц. М.: Наука, 1967.

[19] Белман Р. Введение в теорию матриц. М.: Наука, 1969.

[20] Vere-Jones D. Ergodic properties of nonnegative matrices. I // Pacific J. Math. 1967. V. 22. № 2. P. 361-386.

[21] Vere-Jones D. Geometric ergodicity in denumerable Markov chains // Quart. J. Math. Oxford. Ser. 2. 1962. V. 13. P. 7-28.

[22] Гуревич Б. М. Устойчиво-возвратные неотрицательные матрицы // УМН. 1996. Т. 51. № 3. C. 195-196.

[23] Kendall D. G. Unitary dilations of Markov transition operators and the corresponding integral representations for transition-probability matrices. Probability and statistics. Stockholm: Almqvist and Wiksell, 1959.

[24] Гуревич Б. М., Заргарян А. С. Об условиях существования максималшной меры для счетной символической цепи Маркова // Вестник МГУ. Сер. 1. Матем., мех. 1988.o 5. C. $14-18$.

[25] Gurevich B. M. A variational characterization of one-dimensional countable state Gibbs random fields // Z. Wahrschein. verw. Geb. 1984. V. 68. P. 205-242.

[26] Spitzer F. A variational characterization of finite Markov chains // Ann. Math. Statist. 1972. V. 43. P. 303-307.

[27] Ruelle D. Statistical Mechanics. Rigorous Results. New York: W. A. Benjamin Inc., 1969.

[28] Корнфельд И. П., Синай Я. Г., Фомин С. В. Эргодическая теория. М.: Наука, 1980.

[29] Биллингслей П. Эргодическая теория и информация. М.: Мир, 1969. 
[30] Рохлин В. А. Лекции по энтропийной теории преобразований с инвариантной мерой // УМH. 1967. Т. 22. № 5. С. 3-56.

[31] Ширяев А. Н. Вероятность. М.: Наука, 1980.

[32] Lanford O. E., Ruelle D. Observables at infinity and states with short range correlations in statistical mechanics // Comm. Math. Phys. 1969. V. 13. P. 194-215.

[33] Добрушин Р. Л.Гиббсовские случайные поля для решетчатых систем с парным взаимодействием // Функцион. анализ и его прилож. 1968. Т. 2. № 4. С. 31-43.

[34] Kesten H. Existence and uniqueness of countable one-dimensional Markov random fields // Ann. Probab. 1976. V. 4. P. 557-569.

[35] Гуревич Б. М. Конечные аппроксимации бесконечных неотрицателшных матриц и сходимость равновесных распределений // Докл. АН. 1996. Т. 347. №6. С. 732-735.

[36] Биллингслей П. Сходимость вероятностных мер. М.: Наука, 1977.

[37] Artin E., Mazur B. On periodic points // Ann. Math. 1965. V. 81. P. 82-99.

[38] Ruelle D. Generalized zeta-functions for axiom A basic sets // Bull. Amer. Math. Soc. 1976. V. 82. P. 153-156.

[39] Takahashi Y. Shift with orbit basis and realization of one dimensional maps // Osaka J. Math. 1983. V. 20. P. 599-629.

[40] Bowen R., Lanford O.E. Zeta functions of restrictions of the shift transformation // Proc. Sympos. Pure Math. 1968. V. 14. P. 43-49.

[41] Савченко С. В. Периодические точки счетных топологических марковских цепей // Матем. сб. 1995. Т. 186. № 10. С. 103-140.

[42] Чжун-Кай-Лай. Однородные цепи Маркова. М.: Мир, 1964.

[43] Ruelle D. Statistical mechanics on a compact set with $\mathbb{Z}^{\nu}$-action satisfying expansiveness and specification // Trans. Amer. Math. Soc. 1973. V. 185. P. 237-251.

[44] Алексеев В. М. Символическая динамика. Одиннадщатая математическая школа. Киев: Институт математики АН ССР, 1976.

[45] Георги Г.-О. Гиббсовские меры и фазовые переходы. М.: Мир, 1990.

[46] Phelps R. Convex functions, monotone operators and differentiability. V. 1364. Berlin: Springer-Verlag, 1989 (Lect. Notes in Math.).

[47] Иофффе А. Д., Тихомиров В. М. Теория экстремальных задач. М.: Наука, 1974.

[48] Erdos P., Feller W., Pollard H. A theorem on power series // Bull. Amer. Math. Soc. 1949. V. 55. P. 201-204.

[49] Данфорд Н., Шварц Дж. Т. Линейные операторы. Т. 2. М.: Мир, 1966.

[50] Савченко С. В. О спектрах конечных подматриц бесконечных неразложимых матриц // УМH. 1997. Т. 52. №3. С. 175-176.

[51] Галеева Р.Ф., Чернов Н. И. Условия строго экспоненциального роста числа циклов в счетных ориентированных графах // УМН. 1991. Т. 46. № 4. С. 143-144. 